\title{
Análise Bayesiana para a Superposição de Processos de Poisson Não-Homogêneos Dependentes na Presença de Covariáveis
}

\author{
Willian de Souza Pereira
}

Tese apresentada

aO

Instituto de Matemática e Estatística

$d a$

Universidade de São Paulo

para

obtenção do grau

de

Doutor em Estatística

\author{
Área de Concentração: Estatística \\ Orientador: Prof. Dr. Jorge Alberto ACHCAR
}

São Paulo - Maio - 2005 


\section{Análise Bayesiana para a Superposição de Processos de Poisson Não-Homogêneos Dependentes na Presença de Covariáveis}

Este exemplar corresponde à redação final da tese devidamente corrigida e defendida por Willian de Souza Pereira e aprovada pela comissão julgadora.

São Paulo, 20 de maio de 2005.

Banca examinadora:

- Prof. Dr. Jorge Alberto Achcar (Orientador) - UFSCar

- Prof. Dr. Heleno Bolfarine - IME -USP

- Prof. Dr. Josemar Rodrigues - LFSCar

- Prof. Dr. Josmar Mazucheli - UEM

- Prof. Dra. Reiko Aoki - ICMC - USP 


\section{Epígrafe}

Rir é correr o risco de parecer tolo .

Chorar é correr o risco de parecer sentimental.

Estender a mão é correr o risco de se envolver.

Expor seus sentimentos é correr o risco de mostrar seu verdadeiro eu.

Defender seus sonhos e idéias diante da multidão é correr o risco de perder as pessoas.

Amar é correr o risco de não ser correspondido.

Viver é correr o risco de morrer.

Confiar é correr o risco de se decepcionar.

Tentar é correr o risco de fracassar.

Mas devemos correr os riscos, porque o maior perigo é não arriscar nada.

Há pessoas que não correm nenhum risco, não faz nada, não têm nada e não são nada.

Elas podem até evitar sofrimentos e desilusões, mas não conseguem nada, não sentem nada, não mudam, não crescem, não amam, não vivem . Acorrentadas por suas atitudes, elas viram escravas, privam-se de sua liberdade.

Somente a pessoa que corre riscos é livre!

"Seneca (orador romano que viveu entre $55 \mathrm{aC}$ e $39 \mathrm{dC}$ )" 
Dedico este trabalho aos meus pais José (in memorian) e Maria, as minhas irmãs, particularmente ao José Filho (in memorian), aos meus filhos e a Isa, que me deram todo o apoio que um homem precisa. 


\section{Agradecimentos}

Agradeço...

À Deus, pela vida, pela saúde que permite por mais esta realização em minha vida.

As pessoas com que me relacionei durante esta etapa da minha vida foram tantas que seria quase imposível enumerá-las, por isso só referirei aquelas que mais diretamente estiveram presente.

Ao meu orientador, Prof. Dr. Jorge Alberto Achcar, exemplo de incentivo e confiança.

Aos professores, Dr. Heleno Bolfarine e Dr. Luis Gustavo Esteves pelas valiosas sugestões e comentários dados quando da realização do meu exame de qualificação.

Aos professores do Departamento de Estatística do IME e companheiros de curso, aos funcionários da secretaria da pós-graduação e aos da biblioteca.

Aos amigos, Elmo, Domingo, Ulisses, Alesandro, Ranulfo, Wruck e Iran pelo apóio nos momentos tristes de derrotas, dando-me a força de que precisava para superá-los.

À minha amiga Prof. Dra. Vera pelas valiosas contribuições e estimulos preciosos que me animaram e confortaram na realização dessa tese.

Aos professores e funcionários do Departamento de Estatítica da UFSCar-DEs, a turma do curso de Inferência Bayesiana 2004 pelo companheirismo.

As minhas cunhadas em especial a Sandra e Eunice que sempre estiveram ao meu lado dando força e apoio, e a minha grande família, em especial as minhas irmãs Ana e Benilde que sempre acreditou, sonhou e vivenciou toda as etapas deste trabalho.

À todos os amigos que, de alguma maneira contribuiram para a realização, em especial aos amigos, Silmar, Marcos Primo, Sadao, Milton, Jorge Bazan, Jose Romeo, pela amizade e carinho e pela ajuda no decorrer do trabalho.

Aos colegas do CEFET-MT em especial a Miriam, Mônica, Help, Terezinha e da SEDUC-MT.

Finalmente, ao CNPQ, que subvencionou este trabalho. 


\section{RESUMO}

O principal objetivo deste trabalho é aplicar métodos de Monte Carlo via Cadeias de Markov para obter os sumários a posteriori do parâmetros de interesse de alguns modelos especiais considerados na Teoria de Confiabilidade.

Uma metodologia Bayesiana é desenvolvida para a superposição de dois processos de Poisson não-homogêneos depedentes na presença ou não de covariáveis. Usamos métodos Bayesianos para discrimiar os modelos propostos para os dados de confiabilidade de software.

Uma análise Bayesiana é desenvolvida para processos de Poisson não-homogêneos na presença de um ponto de mudança na função intensidade usando os métodos de Monte Carlo via Cadeias de Markov (MCMIC). Nesta situação, temos interesse obter inferência deste ponto de mudança onde processo de Poisson não-homegêneo muda. Uma ilustração númerica é apresentada com conjunto de dados simulados e reais. 


\section{ABSTRACT}

This study aims to apply Markov Chain Monte Carlo Method in order to obtain posterior summaries of interest for parameters of some special models mentioned in the Reliability Theory.

The Bayesian methodology in this study was developed for the superposition of two dependent non-homogeneous Poisson processes in the presence or not of covariates. Also, It was used Bayesion Methods to discriminate the proposed models for software reliability data.

A Bayesian analysis for the nonhomogeneous Poisson processes in the presence of a change-point for the intensity function is developed using Markov Chain Monte Carlo methods. In this situation, we have interest to get inference on this change-point where nonhomogeneous Poisson process changes. A numerical illustration is presented with simulated data and real data sets. 


\section{Sumário}

1 Preliminares 1

1.1 Introdução aos Processos de Contagem . . . . . . . . . . . . . . . . . 2

1.1.1 O Processo de Poisson . . . . . . . . . . . . . . . . . . 3

1.2 Modelos Utilizados na Modelagem de Confiabilidade de Software . . . . . 5

1.2 .1 Superposição de PPNH . . . . . . . . . . . . . . . . 8

1.2 .2 Construção da Função de Verossimilhança . . . . . . . . . . . . . . 10

1.3 Abordagem Bayesiana . . . . . . . . . . . . . . . . . . . . 13

1.3.1 Método de Monte Carlo em Cadeias de Markov (MCMC) . . . . . 14

1.3.2 Processo de Seleção de Modelos . . . . . . . . . . . . . . . . 16

1.4 Objetivos do Trabalho . . . . . . . . . . . . . . . . . 21

2 Superposição de um Processo Musa e Okumoto com um Processo de Weibull na Presença de Covariáveis $\quad 24$

2.1 Descrição do Modelo Genérico . . . . . . . . . . . . . . . . . . . . . 24

2.2 Uma análise Bayesiana para a superposição de um processo Musa... . . . . 29

2.2.1 Análise Bayesiana para o Modelo M1 (superposição de processos independentes sem a presença de covariáveis) . . . . . . . . . . 31

2.2.2 Análise Bayesiana para o Modelo M2 (superposição de processos dependentes na presença de um covariável) . . . . . . . . . 32

2.2.3 Análise Bayesiana para o Modelo M[3 (superposição de processos dependentes na presença de uma covariável) . . . . . . . . 35

2.2.4 Análise Bayesiana para o Modelo M4 (superposição de processos independentes na presença de uma covariável) . . . . . . . 36

2.3 Exemplo 1 (dados simulados com uma covariável) . . . . . . . . . . . 38 
2.3.1 Modelo M1 (superposição de processos independentes sem a presença de covariáveis) . . . . . . . . . . . . . . . . . . . . 39

2.3.2 Modelo M2 (superposição de processos dependentes na presença de uma covariável $) \ldots \ldots \ldots$. . . . . . . . . . . . . . . . 39

2.3.3 Modelo M3 (superposição de processos dependentes na presença de uma covariável $) \ldots \ldots \ldots \ldots$. . . . . . . . . . . . . . . 41

2.3.4 Modelo M4 (superposição de processos dependentes na presença de uma covariável $\ldots \ldots \ldots$. . . . . . . . . . . . . . . 43

2.4 Exemplo 2 (dados simulados com duas covariáveis) . . . . . . . . . . . . 45

2.4.1 Modelo M5 (superposição de processos dependentes na presença de covariáveis $\ldots \ldots \ldots \ldots \ldots \ldots \ldots$

2.4.2 Modelo M6 (superposição de processos dependentes na presença de covariáveis $\ldots \ldots \ldots \ldots \ldots \ldots$. . . . . . . . . . . . . . 47

2.4.3 Modelo M7 (superposição de processos independentes na presença de covariáveis) . . . . . . . . . . . . . . . . . . 47

2.5 Seleção de Modelos . . . . . . . . . . . . . . . . . . . . . . . . . . . 49

3 Superposição de Processos de Poisson Não-homogêneos Polinomiais Dependentes $\quad \mathbf{5 5}$

3.1 Descrição do modelo genérico . . . . . . . . . . . . . . . . 55

3.2 A Superposição de dois PPNH Polinomiais de Primeiro Grau . . . . . . . 55

3.3 A Superposição de dois PPNH Polinomiais de Segundo Grau . . . . . . . 57

3.4 A Superposição de dois PPNH Polinomiais do Terceiro Grau . . . . . . . . 59

3.5 Análise Bayesiana para a Superposição de dois PPNH Polinomiais... . . . . 61

3.5.1 Modelo M2 (superposição de processos dependentes sem a presença de covariáveis) . . . . . . . . . . . . . . . . . . 6 61

3.5.2 Modelo M3 (superposição de processos dependentes na presença de

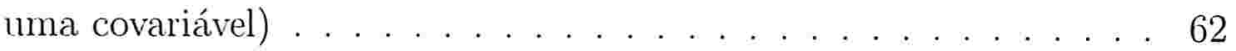

3.5.3 Modelo M4 (superposição de processos dependentes na presença de uma covariável $) \ldots \ldots \ldots$. . . . . . . . . . . . . . . 64

3.6 Aplicações Numéricas . . . . . . . . . . . . . . . . . . . . . . . 66 
3.6 .1 Exemplo 1. . . . . . . . . . . . . . . . . . 66 66

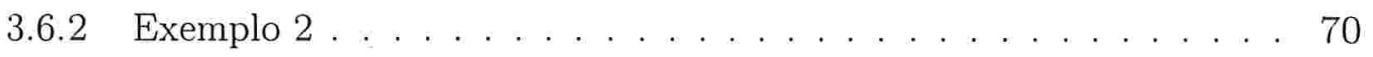

3.6 .3 Exemplo $3 \ldots \ldots \ldots \ldots . \ldots \ldots \ldots$

3.7 Seleção de modelos . . . . . . . . . . . . . . . . . . . . . . . . 80

4 Superposição de Processos de Poisson Não-homogeneos de Lei de Potências 85

4.1 Introdução . . . . . . . . . . . . . . . . . . . . . . . . . . . . 85

4.2 Formulação da Superposição de dois PLP . . . . . . . . . . . . . . . . . . . 86

4.2.1 Modelo M1 (superposição de processos independentes sem a presença de covariáveis

4.2.2 Modelo M2 (superposição de processos dependentes sem a presença

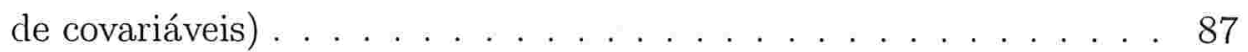

4.3 Superposição de dois PLP Dependentes na Presença de uma Covariável . . 87

4.3.1 Modelo M3 (superposição de processos dependentes na presença de uma covariável $\ldots \ldots \ldots \ldots \ldots$. . . . . . . . . . . . . 87

4.3.2 Modelo M4 (superposição de processos independentes na presença de uma covariável $) \ldots \ldots \ldots$. . . . . . . . . . . . . . . . . 88

4.4 Análise Bayesiana . . . . . . . . . . . . . . . . . . . . . . . . . . . . . . . 88

4.4.1 Modelo M1 (superposição de processos independentes sem a presença de covariáveis $) \ldots \ldots \ldots$. . . . . . . . . . . . . . . . 88

4.4.2 Modelo M2 (superposição de processos dependentes sem a presença de covariáveis) . . . . . . . . . . . . . . . . . . . . . . 90

4.5 Aplicação Numérica . . . . . . . . . . . . . . . . . . . . . . . . . . . . . 92

4.5.1 Modelo M1 (superposição de processos independentes sem a pre-

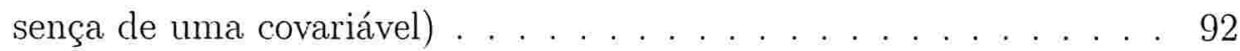

4.5.2 Modelo M2 (superposição de processos dependentes sem a presença (le covariáveis) . . . . . . . . . . . . . . . . . . . . . 93

4.5.3 Modelo M3 (superposição de processos dependentes na presença de uma covariável $\ldots \ldots \ldots \ldots$. . . . . . . . . . . . . . . 94

4.5.4 Modelo M4 (superposição de processos independentes na presença de uma covariável) . . . . . . . . . . . . . . . . . . 96 
4.6 Seleção do modelo . . . . . . . . . . . . . . . . . . . . . . 97

5 Uma Abordagem Bayesiana para um Ponto de Mudança em PPNH 99

5.1 Ponto de Mudança nos Processos de Lei de Potência . . . . . . . . . . . . . . 99

5.2 Ponto de Mudança do Processo de Musa - Okumoto . . . . . . . . . . . . 102

5.3 Aplicações Numéricas . . . . . . . . . . . . . . . . . . . . . . . . 103

5.3.1 Uma Ilustração Numérica com Dados Simulados a partir do Processo Lei de Potência . . . . . . . . . . . . . . . . . . . . . . . 104

5.3.2 Uma Ilustração Numérica com Conjunto de Dados Reais . . . . . . 106

6 Conclusões e Pesquisas Futuras 111

A Diagnósticos de Convergência 112

A.1 Diagnóstico de Geweke . . . . . . . . . . . . . . . . . . . 112

A.2 Diagnóstico de Gelman \& Rubin . . . . . . . . . . . . . . . . . . . . 113

A.3 Diagnóstico de Raftery \& Lewis . . . . . . . . . . . . . . . . . . . . . . 113

A.4 Diagnóstico de Heidelberger \& Welch . . . . . . . . . . . . . . . . . . 114

B A Distribuição de Poisson Bivariada $\quad 115$

$\begin{array}{ll}\text { C Alguns programas } & 116\end{array}$

Referências Bibliográficas $\quad 123$ 


\section{Capítulo 1}

\section{Preliminares}

Para a modelagem estatística de dados de confiabilidade de software a literatura apresenta muitos modelos probabilísticos e várias abordagens: as mais utilizadas são estratégias de modelagem paramétrica para tempos de falhas e as estratégias de contagem de falhas num período de tempo por um processo de Poisson não-homogêneo. O processo de Poisson não-homogêneo (PPNH) é muito usado na modelagem do número de falhas em um intervalo $[0, t]$ em sistemas de reparo e na confiabilidade de software.

$\mathrm{Na}$ literatura, observamos que vários autores tem trabalhado no sentido de unificar os vários modelos propostos por especialistas em software. Uma unificação de muitos dos modelos existentes para modelar dados de confiabilidade de software, é dada por Yang (1994). Ele classifica os modelos de tempos de falha em duas classes:

i) Modelos GOS ou modelos de estatísticas de ordem generalizados;

ii) Modelos RVS ou modelos de estatísticas de valores observados.

Kuo e Yang (1996), introduziram uma análise Bayesiana para modelos de PPNH, considerando o amostrador de Gibbs (ver, por exemplo, Gelfand e Smith, 1990), para obter estimativas para os parâmetros cle interesse. Eles consideram diferentes modelos sob processos de Poisson não-homogeneos (PPNHs) assumindo processos GOS ou RVS. Achcar, Dey e Niverthi (1998), consideram o uso de um supermodelo, a distribuição gama generalizada para modelar a função de valor médio o que generalizou vários resultados obtidos por, Kuo e Yang (1996).

O uso de superposição de PPNHs permite uma grande flexibilidade na modelagem da função de valor médio $m(t)$ incluindo formas crescentes, decrescentes ou em forma 
de banheira (bathtub), (Cid et al.,1999), apresentam uma abordagem Bayesiana para superposição de PPNHs em confiabilidade de software.

Uma das vantagens da superposição de processos PPNH é que podemos ter diferentes formas de função intensidade: funções do tipo banheira, polinômios com picos e vales e funções monótonas simples.

Neste capítulo, na Seção 1.1, apresentamos uma breve introdução aos processos de contagem; na Seção 1.2 introduzimos modelos baseados em PPNHs; na Seção 1.3 apresentamos uma introdução à análise Bayesiana e na Seção 1.4 os objetivos desta tese.

\subsection{Introdução aos Processos de Contagem}

Existe uma extensa literatura sobre modelos de processo de contagem. Esta abordagem nos proporciona ferramentas bastante poderosas capazes de generalizar diversas situações. Alguns conceitos de processos de contagem são introduzidos nesta seção; aspectos mais teóricos são encontrados por exemplo, em Andersen et al. (1993) e Fleming e Harrington (1991).

Um processo de contagem pode ser entendido como um conjunto de variáveis aleatórias $\{N(t): t \geq 0\}$ em que :

(i) $N(0)=0$;

(ii) $N(t)<\infty$;

(iii) $\mathrm{O}$ processo é contínuo à direita;

(iv) $N(t)$ tem apenas descontinuidade de tamanho unitário.

Seja $d N(t)=N(t)-N(t-d t)$ o incremento do processo $N(t)$ num intervalo de comprimento $d t$. Observe que $N(t)$ e $N(t-d t)$ só serão diferentes quando houver falha em $t$, ou seja:

$$
d N(t)=\left\{\begin{array}{l}
1 \text { se ocorreu falha em } t \\
0 \text { se não ocorreu falha em } t .
\end{array}\right.
$$

Sejam $T_{1}, T_{2}, \ldots, T_{n}$ variáveis aleatórias contínuas que representam tempos de falhas distintos de $n$ unidades independentes com f.d.p $f($.) conhecida e de suporte $\Im$. O número de ocorrências do eventos de interesse até o instante $t, t \in \Im$, na $n$ unidades é um processo de contagem definido por

$$
N(t)=\sum_{i=1}^{n} I_{\left\{T_{i} \leq t, d N(t)=1\right\}}
$$


Assim, é definido a variável $Y(t)=\sum_{i=1}^{n} I_{\left\{T_{i} \leq t\right\}}$, que indica o número de unidades de falhas no instante $t$.

Na teoria de processos de contagem, o desenvolvimento no tempo de um processo $N(t)$ é controlado pelo seu processo de intensidade, $\lambda(t)$. Dada a história anterior, o processo $\lambda(t) d t$ é definido pela probabilidade condicional de que $N(t)$ "salte" em um pequeno intervalo de tempo de comprimento $d t$, isto é,

$$
\lambda(t) d t \approx P\left(d N(t)=1 \mid \digamma^{t-}\right)
$$

onde $\digamma^{t-}$ é chamada de filtragem, isto é, uma família crescente de sub- $\sigma$-álgebras definida no espaço amostral. Neste trabalho, é referido à filtragem para denotar apenas a história anterior ao processo, ou seja, tudo o que aconteceu até o instante imediatamente anterior a $t$.

$\mathrm{Na}$ expressão acima, substituindo a probabilidade à esquerda pelo valor esperado de um incremento do proceso $d N(t)=N(t)-N(t-d t)$, obtemos

$$
E\left(d N(t) \mid \digamma^{t-}\right)=\lambda_{0}(t) Y(t) d t
$$

Assim a intensidade do processo $N(t)$ é dada por

$$
\lambda(t)=\lambda_{0}(t) Y(t)
$$

que é conhecida como o modelo de intensidade multiplicativo de Aalen (Aalen, 1978).

\subsubsection{O Processo de Poisson}

Um dos mais importantes tipos de processo de contagem é o processo de Poisson, que é tratado em detalhes na maioria dos livros de processos estocásticos (ver Karlin \& Taylor, 1975). Diz-se que o processo de contagem $\{N(t): t \geq 0\}$ é um processo de Poisson tendo $\operatorname{taxa} \lambda, \lambda>0$. Se

(i) $N(0)=0$;

(ii) O processo tem incrementos independentes;

(iii) O número de eventos em algum intervalo de comprimento $t$ tem distribuição de Poisson com média $\lambda t$. Isto é, para toclo $s, t \geq 0$,

$$
P\{N(t+s)-N(s)=n\}=\frac{e^{-\lambda t}(\lambda t)^{n}}{n !}, \quad n=0,1, \ldots
$$


Note que a condição (iii) mostra que o processo tem incrementos estacionários e também que

$$
E[N(t)]=\lambda t
$$

o que explica porque $\lambda$ é chamado taxa do processo.

Um processo de contagem é dito ter incrementos independentes se os eventos que ocorrem em intervalos de tempos disjuntos são independentes. Por exemplo, o número de eventos que ocorrem no tempo $t$ (isto é, $N(t)$ ) deve ser independente do número de eventos que ocorrem entre os tempos $t$ e $t+s$ (isto é, $N(t+s)-N(t)$ ).

Um processo de contagem é um processo de incrementos estacionários se a distribuição do número de eventos que ocorrem em qualquer intervalo de tempo depende somente do comprimento do intervalo. Em outras palavras, o processo tem incrementos estacionários se o número de eventos no intervalo $\left(t_{1}+s, t_{2}+s\right.$ ] (isto é, $N\left(t_{2}+s\right)-N\left(t_{1}+s\right)$ tem a mesma distribuição que o número de eventos no intervalo $\left(t_{1}, t_{2}\right]$ (isto é, $N\left(t_{2}\right)-N\left(t_{1}\right)$ ) para todo $t_{1}<t_{2}$, e $s>0$.

Assim, para determinar se um processo de contagem arbitrário é realmente um processo de Poisson, deve ser mostrado que as condições (i), (ii) e (iii) estão satisfeitas. As condições (i) e (ii) são facilmente mostradas, mas não fica muito claro mostrar que a condição (iii) é satisfeita, assim uma definição equivalente de um processo de Poisson pode ser útil (ver Ross, 1996).

Assim, como um prefácio para uma segunda definição de um processo de Poisson, deve ser definido o conceito de uma função $f$ sendo $o(h)$ (ordem $h$ ).

A função $f$ é dita ser $o(h)$ se

$$
\lim _{h \rightarrow 0} \frac{f(h)}{h}=0 .
$$

Um processo de contagem $\{N(t): t \geq 0\}$ é dito ser um processo de Poisson com taxa $\lambda, \lambda>0$, se:

(i) $\mathrm{N}(0)=0$;

(ii) O processo tem incrementos independentes e estacionários;

(iii) $P\{N(h)=1\}=\lambda h+o(h)$;

(iv) $P\{N(h) \geq 2\}=o(h)$. 
Um processo de Poisson definido acima onde a taxa $\lambda$ não depende do tempo é chamado processo de Poisson homogêneo.

Diz-se que o processo de contagem $\{N(t): t \geq 0\}$ é um processo de Poisson nãohomogêneo (PPNH) com função de intensidade $\lambda(t), t \geq 0$, se:

(i) $N(0)=0$;

(ii) o processo tem incrementos independentes;

(iii) $P\{N(t+h)-N(t)=1\}=\lambda(t) h+o(h)$;

(iv) $P\{N(t+h)-N(t) \geq 2\}=o(h)$.

A importância do processo de Poisson não-homogêneo (PPNH) reside no fato de não requerer a condição de incrementos estacionários.

Definição: A função de valor médio, $m(t)$ é definida por,

$$
m(t)=\int_{0}^{t} \lambda(s) d s .
$$

Para indicar que $\{N(t), t \geq 0\}$ é um processo PPNH com função intensidade $\lambda(t)>$ 0, escreveremos:

$$
N(t) \sim \operatorname{Poisson}(m(t))
$$

onde $m(t)$ é dada por (4.5).

Assim podemos mostrar que

$P\{N(t+s)-N(t)=n\}=\frac{\exp \{-m(t+s)-m(t)\}[m(t+s)-m(t)]}{n !}, \quad n \geq 0$, isto é, $N(t+s)-N(t)$ tem distribuição de Poisson com média $m(t+s)-m(t)$ (ver por exemplo, Ross, 1996).

A flexibilidade do modelo está relacionada com as diferentes formas que a função de intensidade pode assumir. Se a função de intensidade é constante, então o PPNH se reduz ao caso de processo de Poisson homogêneo (PPH), isto é, $\lambda(\mu)=\lambda$ para todo $\mu$. Assim $m(t)=\lambda t$.

\subsection{Modelos Utilizados na Modelagem de Confiabili- dade de Software}

Na literatura, observamos que vários autores tem trabalhado no sentido de unificar os vários modelos propostos por especialistas em software. 
Langberg e Singpurwalla (1985), consideram os modelos G.O. (Goel e Okumoto, 1979) e L.V. (Littlewood e Verall, 1973), como generalização do modelo J.M (Jelinsky e Moranda, 1972). Outra generalização é proposta por Miller (1986), considerando os tempos entre falhas como variáveis aleatórias com distribuições exponenciais, mas não idênticas.

Uma unificação da maioria dos modelos existentes para modelar dados de confiabilidade de software, é dada por Yang (1994), onde ele classifica os modelos de tempos de falhas em duas classes: Modelos GOS ou modelos de estatísticas de ordem generalizados e Modelos RVS ou modelos de estatísticas de valores observados.

Os modelos GOS assumem que existe um número desconhecido de falhas $N$ no começo do processo de debugging ou teste e modela as épocas observadas de falha como as primeiras $n$ estatísticas de ordem tomadas das $N$ observações i.i.d. com f.d.p. com suporte em $R^{+}$. Assumimos que neste modelo ao repararmos alguma falha não são introduzidas novas falhas.

Os modelos RVS permitem a introdução de novas falhas após o reparo de uma falha. Os modelos RVS assumem que os tempos de falhas são estatísticas observadas i.i.d. com suporte $R^{+}$.

Sob o ponto de vista dos processos de contagem, a teoria unificada proposta por Yang (1994) considera a classificação dos modelos de acordo com o número de falhas: finito ou infinito. Tanto para os modelos GOS quanto para os modelos RVS, $N(t)$, está relacionado com um PPNH. No caso GOS estamos supondo que $N$ tem distribuição de Poisson com média $\theta$. Assim, temos que $N(t)$ é um PPNH com função de valor médio

$$
m(t)=\theta F(t)
$$

onde $F$ é a função de distribuição acumulada (F.D.A.) com f.d.p., $f$. Obviamente, neste caso,

$$
\lim _{t \rightarrow \infty} m(t)<\infty
$$

Yang (1994) chama este tipo de modelos de Processos PPNH-I.

Os modelos para os quais ocorre que 


$$
\lim _{t \rightarrow \infty} m(t)=\infty
$$

são chamados de processos PPNH-II.

Dado um modelo RVS derivado a partir de amostras i.i.d. de f, podemos provar que o processo correspondente $N(t)$ é do tipo PPNH-II com função de valor médio

$$
m(t)=-\log (1-F(t))
$$

A implicação inversa pode ser estabelecida da seguinte forma: tempos de falha de um PPNH-II com função intensidade $\lambda(t)$ correspondem a um modelo RVS derivado de observações i.i.d. com f.d.p. comum dada por:

$$
f(t)=\lambda(t) \exp \left\{-\int_{0}^{t} \lambda(u) d u\right\} .
$$

$\mathrm{Na}$ literatura existem vários modelos que envolvem processo PPNH para modelar sistema de reparos ou confiabilidade de software; os mais importantes que serão utilizados neste trabalho são os seguintes:

Modelo de Weibull (Duane e Crow, 1966)

$\lambda(t)=\alpha \beta t^{\beta-1}, \quad t \geq 0, \quad \alpha>0, \quad \beta>0$.

Modelo de Goel e Okumoto (1979).

$\lambda(t)=\alpha \beta t^{\beta t}, \quad t \geq 0, \quad \alpha>0, \quad \beta>0$.

Modelo de Musa e Okumoto (1984).

$\lambda(t)=\frac{\alpha}{\beta+t}, \quad t \geq 0, \quad \alpha>0, \quad \beta>0$.

Modelo de Kuo e Yang (1996) (Modelo Polinomial).

$\lambda(t)=\beta_{0}+\beta_{1} t+\cdots+\beta_{J-1} t^{J-1}, \quad t \geq 0, \quad \beta_{i}>0, \quad i=1, \ldots, J-1$.

Modelo de Dharmadhikari,Naik-Nimbalkar e Bhyri (1989), (Modelo de lei de potência).

$\lambda(t)=\frac{\beta}{\theta}\left(\frac{t}{\theta}\right)^{\beta-1}, \quad t \geq 0, \beta>0, \quad \theta>0$.

Notemos que este último modelo é caso particular do modelo de Weibull com uma simples reparametrização. 


\subsubsection{Superposição de PPNH}

Consideramos a modelagem do número de falhas de um software usando um processo pontual para contar o número de falhas (ver por exemplo, Musa, Ianino e Okumoto, 1987).

Seja $M(t)$ o número acumulado de falhas que são observadas durante o tempo $(0, t]$. Suponhamos que $M(t)$ é modelado por um PPNH com função intensidade

$$
\lambda(t)=\frac{d E[M(t)]}{d t},
$$

a qual assumimos uma função monótona de $t$ (ver Musa e Okumoto, 1984 ou Cox e Lewis, 1966).

Uma alternativa ao uso de funções monótonas é considerar a superposição de vários PPNHs independentes.

Definição: Seja $M_{1}(t)$ e $M_{2}(t)$ dois processos independentes, cada um tendo a taxa $\lambda$ e $\mu$ respectivamente. $\mathrm{O}$ conjunto de pontos de falhas de um processo de superposição é definido como a união dos dois processos $M_{1}(t)$ e $M_{2}(t)$ e é dado por:

$$
M(t)=M_{1}(t)+M_{2}(t)
$$

então o processo $M(t)$ é chamado de superposição dos processos $M_{1}(t)$ e $M_{2}(t)$ (ver Figura 1.1).

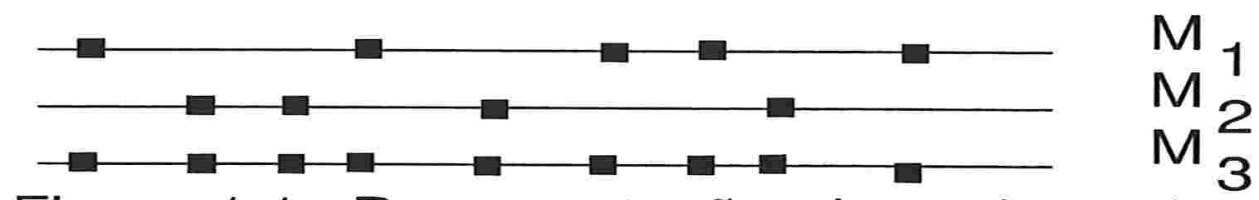

Figura 1.1: Representação do conjunto de pontos de falha da superposição de dois processos

Assim $M(t)$ é um processo de Poisson com taxa $\nu=\lambda+\mu$.

Seja $M_{j}(t)$ o PPNH que representa o número acumulado de falhas em $(0, t]$ para o $j$ ésimo processo componente com função de intensidade $\lambda_{j}\left(t \mid \beta_{j}\right)$, cuja forma assumimos conhecida, mas que inclui o vetor de parâmetros $\beta_{j}$ desconhecido. 
Assumimos também que os $M_{j}(t), j=1, \ldots, J$ são mutuamente independentes. Dai podemos provar que o processo,

$$
M(t)=\sum_{j=1}^{J} M_{j}(t),
$$

o qual conta o número total de falhas no intervalo $(0, t]$ é um PPNH com função intensidade,

$$
\lambda(t \mid \beta)=\sum_{j=1}^{J} \lambda_{j}\left(t \mid \beta_{j}\right),
$$

onde $\beta=\left\{\beta_{1}, \beta_{2}, \ldots, \beta_{J}\right\}$ (ver Çinlar, 1975, pp.87-88).

Seja $M_{j}(t)$ o $j$-ésimo processo componente com função intensidade $\lambda_{j}\left(t \mid \beta_{j}\right)$ assumindo uma superposição de $J$ PPNHs, cuja forma assumimos conhecida, a função intensidade de $M(t)$ é dada por:

$$
\lambda(t \mid \beta)=\frac{d E[M(t)]}{d t}=\lambda_{1}\left(t \mid \beta_{1}\right)+\ldots+\lambda_{J}\left(t \mid \beta_{J}\right) . .
$$

Um caso considerado na literatura (ver, por exemplo, Kuo e Yang, 1996) é dado quando os PPNHs $M_{j}(t) j=1, \ldots, J$ são mutualmente independentes.

Seja $M_{j}(t) j=1,2$ o número de falhas em $(0, t)$ em $(1.11)$, se $M_{1}(t)$ e $M_{2}(t)$ são PPNHs então $M(t)$ é também um PPNH com função intensidade $\lambda(t)=\lambda_{1}\left(t \mid \beta_{1}\right)+\lambda_{2}\left(t \mid \beta_{2}\right)$ onde $\beta=\left\{\beta_{1}, \beta_{2}\right\}$ (ver por exemplo, Yang e Kuo, 1999). Neste caso assumimos que os subsistemas são independentes. Alguns casos especiais de modelos de superposição são dados por:

(i) $\lambda(t)=\alpha_{1} \beta_{1} t^{\alpha_{1}-1}+\alpha_{2} \beta_{2} t^{\alpha_{2}-1}$, (duas funções intensidades de Weibull)

(ii) $\lambda(t)=\frac{\alpha_{1}}{t+\alpha_{2}}+\beta_{1} \beta_{2} t$, (Musa-Okumoto; 1984)

(iii) $\lambda(t)=\frac{\alpha_{1}}{t+\alpha_{2}}+\alpha_{1} t+\alpha_{3}$, (Gaver and Acar, 1979)

(iv) $\lambda(t)=\frac{\alpha_{1}}{t+\alpha_{2}}+\beta t, \quad($ Hjorth, 1980)

(v) $\lambda(t)=\beta_{0}+\beta_{1} t$ (função polinomial primeiro grau)

(vi) $\lambda(t)=\frac{\beta_{1}}{\theta_{1}}\left(\frac{t}{\theta_{1}}\right)^{\beta_{1}-1}+\frac{\beta_{2}}{\theta_{2}}\left(\frac{t}{\theta_{2}}\right)^{\beta_{2}-1}$ (lei de potência). 
Observe que em (iv), $\lambda_{1}(t)$ é a função intensidade para o processo de Musa-Okumoto, e $\lambda_{2}(t)$ é um caso especial de intensidade do processo de Weibull que é usado na deterioração da confiabilidade.

\subsubsection{Construção da Função de Verossimilhança}

Nesta subseção, apresentamos a construção da função de verossimilhança para um PPNH.

Suponha que $m(t)$ é indexada por parâmetros desconhecidos $\theta$ e $\beta$, onde $\theta$ é um número real não-negativo e $\beta$ pode ser um vetor de parâmetros desconhecidos. Por simplicidade, escrevemos $m(t \mid \theta, \beta)$.

Dado o modelo de tempos truncado, o número de falhas observadas em $[0, t]$, as épocas ordenadas dos $n$ tempos de falhas são denotados por:

$$
x_{1}, x_{2}, \ldots, x_{n}
$$

O conjunto de dados $D_{t}$ pode ser escrito na forma:

$$
D_{t}=\left\{n, x_{1}, x_{2}, \ldots, x_{n} ; t\right\}
$$

Na sua tese, Yang(1994), apresenta duas opções para obter a verossimilhança:

Método 1

Dado o modelo de tempo truncado, o número de falhas observadas em $(0, t]$ é uma variável aleatória denotada por $n$.

Dado $M(t)=n$, os tempos

$$
x_{1}<x_{2}<\cdots<x_{n}<t
$$

Tem a mesma distribuição das estatísticas de ordem de v.a.i.i.d com F.D.A. comum dacla por,

$$
F(w)=\frac{m(w)}{m(t)} \quad 0 \leq w \leq t
$$


Assim, a função de verossimilhança é dada por

$$
\begin{aligned}
L\left(\theta, \beta \mid \mathfrak{D}_{\mathfrak{t}}\right) & =P\left[\mathfrak{D}_{\mathfrak{t}} \mid M(t)=n\right] P[M(t)=n] \\
& =n !\left[\prod_{i=1}^{n} \frac{\lambda\left(x_{i}\right)}{m(t)}\right] \frac{e^{-m(t)}[m(t)]^{n}}{n !} \\
& =\left[\prod_{i=1}^{n} \lambda\left(x_{i}\right)\right] e^{-m(t)}
\end{aligned}
$$

onde $\beta$ é o vetor de parâmetros.

Método 2

A sequência de tempos

$$
x_{1}<x_{2}<\cdots,
$$

é um PPNH, e a sequência

$$
m\left(x_{1}\right)<m\left(x_{2}\right)<\cdots,
$$

é um PPNH com taxa 1, logo os tempos entre as chegadas são distribuídos exponencialmente com taxa 1 .

Assim,

$$
P\left[m\left(X_{n}\right) \geq m\left(X_{n-1}+t\right)\right]=P\left[X_{n}-X_{n-1}>t\right] .
$$

Logo,

$$
\begin{aligned}
P\left[X_{n}-X_{n-1}>t \mid X_{1}, \ldots, X_{n-1}\right] & =P\left[m\left(X_{n}\right) \leq m\left(X_{n-1}+t\right) \mid X_{1}, \cdots, X_{n-1}\right] \\
& =\exp \left\{-\left(m\left(X_{n-1}+t\right)-m\left(X_{n}\right)\right)\right\} \\
& =P\left[X_{n}-X_{n-1}>t \mid X_{n-1}\right] .
\end{aligned}
$$

Além disso,

$$
\begin{aligned}
f_{X_{1}}\left(x_{1}\right) & =\lambda\left(x_{1}\right) \exp \left\{-m\left(x_{1}\right)\right\} \\
f_{X_{n} \mid X_{n-1}}\left(x_{n} \mid x_{n-1}\right) & =\lambda\left(x_{n}\right) \exp \left\{-\left(m\left(x_{n}\right)-m\left(x_{n-1}\right)\right\} .\right.
\end{aligned}
$$

Portanto, a função de verossimilhança para o modelo de tempo truncado é dado por: 


$$
\begin{aligned}
L\left(\theta, \underline{\beta} \mid \mathfrak{D}_{\mathfrak{t}}\right) & =f\left(x_{1}, x_{2}, \ldots, x_{n} \mid t\right) \\
& =f_{X_{1}}\left(x_{1}\right) f_{X_{2}} \mid X_{1}\left(x_{2} \mid x_{1}\right) \cdots f_{X_{n} \mid X_{n-1}}\left(x_{n} \mid x_{n-1}\right) P\left[X_{n+1}>t \mid x_{n}\right] .
\end{aligned}
$$

e daí obtemos a mesma expressão (1.16).

No caso do modelo de falhas truncado obtemos uma expressão (1.16) análoga, que é dada por:

$$
L\left(\theta, \beta \mid \mathfrak{D}_{\mathrm{t}}\right)=e^{-m(t)} \prod_{i=1}^{n} \lambda\left(x_{i}\right),
$$

onde $m(t)=\int \lambda(u) d u$ é a função do valor médio.

Essa teoria é desenvolvida em muito livros textos, por exemplo, Basawa e Prakasa Rao (1980), Cox e Lewis (1966) e Crowder, Kimber, Smith, e Sweeting (1991). Expressões similares podem ser obtidas para o modelo de falha truncado (observado até a n-ésima falha).

Assumindo o modelo de falha truncado e a superposição de dois processos de Poisson não-homogêneos $M_{1}(t)$ e $M_{2}(t)$ com uma distribuição de Poisson bivariada (ver Rodrigues, et al., 2002), a função de verossimilhança para $\underline{\beta}$ e $\rho$ (o parâmetro de correlação) é dada por

$$
\begin{aligned}
L(\underline{\beta}, \rho) & =\prod_{i=1}^{n}\left[\lambda_{1}\left(t_{i}\right)+\lambda_{2}\left(t_{i}\right)\right] \cdot\left[1-\frac{\rho W\left(t_{i}\right)}{2}\right] \\
& \times \exp \left\{-\left[m_{1}(t)+m_{2}(t)-\rho\left(m_{1}(t) m_{2}(t)\right)^{1 / 2}\right]\right\}
\end{aligned}
$$

onde $\lambda_{1}\left(t_{i}\right)=\lambda_{1}\left(t_{i} \mid \underline{\beta_{1}}\right), \lambda_{2}\left(t_{i}\right)=\lambda_{2}\left(t_{i} \mid \underline{\beta_{2}}\right)$ são as respectivas intensidades componentes,

$$
W\left(t_{i}\right)=\frac{\lambda_{1}\left(t_{i}\right)\left[\frac{m_{2}\left(t_{i}\right)}{m_{1}\left(t_{i}\right)}\right]^{1 / 2}+\lambda_{2}\left(t_{i}\right)\left[\frac{m_{1}\left(t_{i}\right)}{m_{2}\left(t_{i}\right)}\right]^{1 / 2}}{\lambda_{1}\left(t_{i}\right)+\lambda_{2}\left(t_{i}\right)}, \quad i=1,2, \ldots, n
$$

$n$ é o número de observações na amostra e $m_{1}(t)$ e $m_{2}(t)$ são as funções de valor médio correspondentes $\left(m_{j}=\int \lambda_{j}(t) d t\right)$. O parâmetro $\rho(0 \leq \rho \leq 1)$ é o parâmetro de correlação entre os dois PPNHs. Observe que se $\rho=0$, temos a superposição de dois PPNHs independentes.

Para obter inferências sobre $\underline{\beta}=\left(\beta_{1}, \beta_{2}\right)$ e $\rho$ vamos usar métodos Bayesianos. 


\subsection{Abordagem Bayesiana}

Nesta seção apresentamos alguns conceitos básicos para que os modelos possam ser abordados numa perspectiva Bayesiana. A abordagem Bayesiana é adequada quando ocorrem situações onde os dados avaliados são incompatíveis com modelos simples e outros modelos mais realísticos e, consequentemente modelos mais complexos são necessários.

A implementação de técnicas Bayesianas para tais modelos complexos pode resultar em distribuições a posteriori de difícil tratamento analítico, como por exemplo, o grande número de parâmetros a serem estimados, a dificuldade na obtenção das densidades marginais de forma analítica, ou também quando as distribuições a priori e a posteriori não são conjugadas. Nesses casos, os métodos analíticos de aproximação não são indicados, e métodos de aproximação numérica são necessários para estimação dos parâmetros de interesse. Os métodos analíticos de aproximação são eficientes computacionalmente; porém são baseados em resultados assintóticos e na suposição de normalidade; também são mais difíceis para programar à medida que o número de parâmetros aumenta. Até recentemente, duas abordagens têm sido dadas a esse problema, a aproximação de Laplace (Tierney, Kass \& Kadane, 1989) e a integração de Monte Carlo via Importance Sampler (ver por exemplo Ripley, 1987).

Assim como na inferência clássica, a inferência Bayesiana é feita na presença de observações $\mathrm{x}$ descritas através de uma distribuição de probabilidades $f(x \mid \underline{\theta})$. A quantidade $\underline{\theta}$ serve como indexador da família de distribuições das observações representando características de interesse que se deseja conhecer para poder ter uma descrição completa do processo.

Dado o conjunto dos tempos de falhas $\mathrm{x}=\left(x_{1}, \ldots, x_{k}\right)$ obtidos sobre um modelo paramétrico $f(x \mid \underline{\theta}), \underline{\theta}=\left(\theta_{1}, \cdots, \theta_{k}\right)$, o procedimento de inferência Bayesiana é baseado na forma familiar do teorema de Bayes, isto é,

$$
p(\underline{\theta} \mid \mathbf{x})=\frac{l(\underline{\theta} ; x) \pi(\underline{\theta})}{\int l(\underline{\theta} ; x) \pi(\theta) d \theta}
$$

que descreve a distribuição a posteriori $p(\underline{\theta} \mid x)$, combinando-se a função de verossimilhança $l(\underline{\theta} ; x)$ com a priori $\pi(\theta)$. Se o interesse é centrado em algum componente de $\underline{\theta}$, digamos $\theta_{i}(i=1, \ldots, k)$, a correspondente distribuição marginal pode ser facilmente 
obtida integrando-se a distribuição conjunta $p(\underline{\theta} \mid \mathbf{x})$, isto é,

$$
p\left(\theta_{i} \mid x\right)=c \int l(\underline{\theta} ; x) \pi(\underline{\theta}) d \theta_{-i},
$$

onde o subscrito $-i$ implica a integração de todos os componentes exceto $\theta_{i}$. Se inferências sumárias na forma de esperanças a posteriori são requeridas, elas são obtidas por

$$
E(\psi(\underline{\theta}) \mid x)=\int \psi(\underline{\theta}) p(\underline{\theta} \mid x) d \theta
$$

para uma escolha de $\psi(\theta)$ de interesse.

Nas últimas décadas, alguns métodos numéricos têm sido desenvolvidos para resolver as questões mencionadas acima. Os métodos numéricos baseados em amostragem englobam os métodos de integração simples de Monte Carlo, os métodos de reamostragem por importância e os métodos de Monte Carlo via Cadeias de Markov (MCMC). Estes são mais simples para implementação e não apresentam restrições quanto ao número de parâmetros a serem estimados.

\subsubsection{Método de Monte Carlo em Cadeias de Markov (MCMC)}

O método MCMC é uma forma de integração Monte Carlo. A idéia é simular uma cadeia de Markov irredutível aperiódica cuja distribuição estacionária é a distribuição de interesse $p(\underline{\theta})$. Para os Bayesianos, isso é a densidade a posteriori $p(\underline{\theta} \mid x)$. Existem dois métodos de gerar cadeia de Markov com distribuição estacionária especificada. Um deles que tem sido usado por muitos anos em estatística fisica: é o algoritmo de Metropolis (Metropolis et al., 1953). Hastings (1970) mostra uma generalização do algoritmo Metropolis. O outro método é o Gibbs Sampler (Geman \& Geman, 1984), que foi introzido na literatura estatística por Gelfand \& Smith (1990). É importante observar que o uso desses algoritmos, em geral, é necessário para casos onde a geração não-iterativa da distribuição da qual se deseja obter uma amostra for muito complicada ou custosa. Na subseção seguinte, é descrito alguns algoritmos comumente utilizados nos diversos trabalhos publicados até agora. 


\section{Amostrador de Gibbs (Gibbs Sampling)}

O Amostrador de Gibbs é um método MCMC. Esta técnica é usada para gerar variáveis aleatórias de uma distribuição sem utilizar diretamente sua densidade de probabilidade. Ele foi desenvolvido por Geman \& Geman (1984), como ferramenta para modelos de processamento de imagens. Gelfand \& Smith (1990) apresentaram aplicações interessantes em uma grande variedade de problemas estatísticos. Casella \& George (1992) ilustraram a teoria com dois casos: um bivariado e outro com dimensão maior do que 2. Este método é essencialmente um processo iterativo de amostragem de uma cadeia de Markov cujo núcleo de transição é formado pelas condicionais completas. Para descrever de forma geral, considere o vetor aleatório $\underline{\theta}=\left(\theta_{1}, \ldots, \theta_{k}\right)$ com distribuições condicionais completas para que os $k$ parâmetros estejam disponíveis. Essas distribuições são denominadas por $\pi\left(\theta_{1} \mid \theta_{2}, \theta_{3}, \ldots, \theta_{k}\right), \pi\left(\theta_{2} \mid \theta_{1}, \theta_{3}, \ldots, \theta_{k}\right), \ldots, \pi\left(\theta_{k} \mid \theta_{1}, \theta_{2}, \ldots, \theta_{k-1}\right)$. O algoritmo segue os seguintes passos:

Passo 0: Arbitre um valor inicial $\theta^{0}=\left(\theta_{1}^{0}, \ldots, \theta_{k}^{0}\right)$ e inicialize o contador de iteração $i=1$

Passo 1: Obtenha um novo valor $\theta^{(i)}=\left(\theta_{1}^{(i)}, \ldots, \theta_{k}^{(i)}\right)$ a partir de $\theta^{(i-1)}$ através de sucessivas gerações de valores:

$$
\begin{aligned}
& \operatorname{gerar} \theta_{1}^{(i)} \sim \pi\left(\theta_{1} \mid \theta_{2}^{(i-1)}, \ldots, \theta_{k}^{(i-1)}\right) \\
& \text { gerar } \theta_{2}^{(i)} \sim \pi\left(\theta_{2} \mid \theta_{1}^{(i)}, \theta_{3}^{i-1}, \ldots, \theta_{k}^{(i-1)}\right) \\
& \vdots \\
& \operatorname{gerar} \theta_{k}^{(i)} \sim \pi\left(\theta_{k} \mid \theta_{1}^{(i)}, \theta_{2}^{i}, \ldots, \theta_{k-1}^{(i)}\right)
\end{aligned}
$$

Passo 2: Incremente $i$ e volte para o passo 1 até atingir a convergência.

À medida que o número de iterações aumenta, a cadeia se aproxima de sua condição de equilíbrio. Assim, assume-se que a convergência é atingida em uma iteração cuja distribuição esteja arbitrariamente próxima da distribuição de equilíbrio. Replicações paralelas independentes de todo o algoritmo acima $\mathrm{N}$ vezes produz $\mathrm{N}$ conjuntos de vetores dos parâmetros e, então, para cada elemento de $\theta$, obtemos uma amostra simulada de tamanho $\mathrm{N}$ da distribuição a posteriori conjunta. 


\section{Algoritmo de Metropolis-Hastings}

Quando as distribuições condicionais a posteriori não são facilmente identificadas como possuidoras de uma forma padrão (normal, gama etc.), que impossibilita a geração direta a partir destas distribuições, usa-se o algoritmo Metropolis (Metropolis et al., 1953). Uma generalização do algoritmo dado por Hastings (1970) é descrito aqui. Esta técnica requer uma densidade de transição $q\left(\theta, \theta^{*}\right)$, que, não necessariamente, tem probabilidade de equilíbrio $\pi$, mas que represente uma regra de passagem que defina uma cadeia. Considere, também, a probabilidade de aceitação $p\left(\theta^{(r-1)}, \theta^{*}\right)$ definida abaixo. O algoritmo segue os seguintes passos:

Passo 0: arbitre um valor inicial $\theta=\theta^{(0)}$; inicialize o contador de iteração $r=1$;

Passo 1: mova a cadeia para um novo valor de $\theta^{*}$ gerado da densidade $q\left(\theta^{(r-1)}, \theta^{*}\right)$;

Passo 2: gere u da uniforme $(0,1)$;

Passo 3: aceite o valor $\theta^{*}$ gerado se

$$
u=p\left(\theta^{(r-1)}, \theta^{*}\right)=\min \left(1, \frac{\pi\left(\theta^{*}\right) q\left(\theta^{*}, \theta^{(r-1)}\right.}{\pi\left(\theta^{r-1}\right) q\left(\theta^{(r-1)}, \theta^{*}\right)}\right) .
$$

Caso contrário, fique $\operatorname{com} \theta^{(r)}=\theta^{(r-1)}$;

Passo 4: incremente $r$ e volte para o passo 1.

Para $r$ suficientemente grande, $\theta^{(r)}$ é uma amostra da distribuição a posteriori $\pi(\theta)$.

Para o caso vetorial $\theta=\left(\theta_{1}, \theta_{2}, \ldots, \theta_{p}\right)$, teremos a densidade de transição dada por $q\left(\theta, \theta^{*}\right)$ e uma probabilidade de aceitação dada por $p\left(\theta^{(r-1)}, \theta^{*}\right)$. Depois, deve-se proceder como na forma acima.

\subsubsection{Processo de Seleção de Modelos}

O processo de seleção de um modelo adequado se torna um problema importante na estatística. Sob o enfoque Bayesiano, a literatura sobre a adequação ou processo de seleção do modelo apresenta muitas abordagens, dentre os quais o uso do fator de Bayes. Várias modificações do fator de Bayes são apresentadas na literatura (ver por exemplo, Aitkin, 1981; Berger e Perichi, 1992 ou Spiegelhalter e Smith, 1982) para a situação onde usamos 
distribuições a priori não-informativa impróprias. Geisser e Eddy (1979) sugerem uma abordagem preditiva baseada em métodos de validação cruzada para obter o pseudo-fator de Bayes.

\section{Critério de Informação Bayesiana}

O fator de Bayes é muito usado para a comparação de modelos (ver por exemplo, Kass e Raftery, 1995). Dey et al., (1999) apresentam a relação entre o fator de Bayes e outros critérios de escolha de modelos conhecidos, por exemplo, o critério de informação Akaike (AIC) e o critério de informação Bayesiano(BIC)(Schwartz, 1978). Spiegelhalter et al., (2002) generaliza o critério AIC e definem como critério DIC (Deviance Information Criterion). A seguir, vamos descrever estes métodos de seleção de modelos.

\section{Critério de Informação Akaike}

Este critério é baseado em considerações frequentistas de eficiência assintótica. A função genérica que calcula o critério de informação Akaike para um ou mais ajuste do modelo é dada por

$$
\widehat{A I C}=-2 \ln \left(\max _{\Theta_{k}}(L[\theta])\right)+2 k,
$$

onde $\Theta_{k}$ é o espaço de parâmetro correspondente a $\mathrm{k}$ parâmetros. Valores menores do AIC indicam o melhor ajuste do modelo. Quando a informação contida na distribuição a priori aumentar na mesma razão que a informação contida na verosimilhança, isto é, esta situação não é realista do ponto de vista da metodologia Bayesiana e, como tal, não é razoável a sua utilização (ver, Paulino et al., 2003, pp. 352-353).

\section{Critério de Informação Bayesiana}

O critério de informação Bayesiano é daclo por,

$$
\widehat{B I C}=-2 \ln \left(\max _{\Theta_{k}}(L[\theta])\right)+k \ln (\text { nobs })
$$


onde $\Theta_{k}$ é o espaço de parâmetro correspondente a $\mathrm{k}$ parâmetros e nobs é o número de observações. Maiores valores do BIC indicam o melhor ajuste do modelo.

Kass e Wasserman (1995) afirmam que o número de observações que aparece no termo que penaliza a verossimilhança em (1.24) deve ser escolhido cuidadosamente, sem sacrificar as propriedades assintoticas.

\section{Crítério do DIC}

Spiegelhalter et al., (2002) generalizam o critério AIC baseada na distribuição a posteriori dada por

$$
D_{i}(\theta)=-2 \ln \left(\frac{f\left(\underline{x} \mid \theta, M_{i}\right)}{h(\underline{x})}\right), \quad i=1,2
$$

onde $h(\underline{x})$ é uma função só dos dados e não tem impacto na escolha do modelo.

A função genérica que calcula está informação é dada por

$$
\widehat{D I C}_{i}=\bar{D}_{i}+p_{D_{i}}
$$

onde $\bar{D}_{i}=E_{\underline{\theta}_{i}} \mid \underline{x}_{i}, M_{i}\left[D_{i} \mid \underline{\theta}_{i}\right]$ é a média a posteriori da função deviance e $p_{D_{i}}$ é o número efetivo de parâmetros do modelo $i$ em estudo, calculado da seguinte forma

$$
p_{D_{i}}=E_{\left(\theta_{i} \mid x, M_{i}\right)}\left[D_{i}\left(\theta_{i}\right)\right]-D_{i}\left[E_{\left(\theta \mid x, M_{i}\right)}\left[D_{i}(\theta)\right]\right] .
$$

Menores valores do DIC indicam o melhor ajuste do modelo.

\section{Critério da Densidade Preditiva}

Sob o critério das densidades preditivas, escolher um entre dois modelos paramétricos cada um denotado por sua densidade preditiva $f\left(\underline{t} \mid \underline{\beta}, M_{i}\right)$ ou por sua função de verossimilhança $L\left(\beta_{\mathbf{i}} \mid \underline{m}, M_{i}\right), i=1,2$, baseado na densidade preditiva sob um dos dois modelos. Supor que $w_{i}$ é a probabilidade de selecionar o modelo $M_{i}, i=1,2$ e $f\left(\underline{t} \mid M_{i}\right)$ é a distribuição preditiva (verossimilhança preditiva) do modelo $M_{i}$, definida por:

$$
f\left(\underline{t} \mid M_{i}\right)=\int f\left(\underline{t} \mid \beta_{\mathrm{i}}, M_{i}\right) \pi\left(\beta_{i} \mid M_{i}\right) d \beta_{\mathrm{i}}
$$


onde $\pi\left(\beta_{\mathrm{i}} \mid M_{i}\right)$ é a densidade a priori sobre o modelo $M_{i}$. Se $\underline{t}_{0}$ denota os dados observados, então escolhemos o modelo que tem maior valor $w_{i} f\left(\underline{t_{0}} \mid M_{i}\right)$.

Frequentemente considera-se $w_{i}=0.5, i=1,2$ é o fator de Bayes de $M_{1}$ com respeito a $M_{2}$ é definido por:

$$
B_{12}=\frac{f\left(\underline{t}_{0} \mid M_{1}\right)}{f\left(\underline{t}_{0} \mid M_{2}\right)}
$$

A verossimilhança preditiva (1.28) pode ser aproximada por um estimador de Monte Carlo usando $S$ amostras geradas a partir da densidade a priori $\pi\left(\underline{\beta_{\mathrm{i}}} \mid M_{i}\right)$, isto é,

$$
\hat{f}\left(\underline{t} \mid M_{i}\right)=\frac{1}{S} \sum_{s=1}^{S} f\left(\underline{t} \mid \beta_{\mathrm{i}}^{(\mathbf{s})}, M_{i}\right),
$$

onde $\beta_{\mathbf{i}}^{(\mathrm{s})}$ é a s-ésima amostra gerada do vetor $\beta_{\mathbf{i}}$.

Podemos notar que,caso a distribuição a priori $\pi\left(\beta_{\mathrm{i}} \mid M_{i}\right)$ seja imprópria, o que pode acontecer quando consideramos densidades a priori não informativas, $f\left(D \mid M_{i}\right)$ também será impropria e consequentemente (1.28) não pode ser usada como critério de comparação, uma vez que não se tem uma razão de probabilidades. Esse fato pode restringir o uso do fator de Bayes à classe das distribuições a priori próprias, ou ainda a modelos razoalvemente simples, o que em geral não ocorre na prática, principalmente com relação a modelos sobrevivência ou de confiabilidade.

No sentido de superar restrições associadas ao fator de Bayes, muitos trabalhos recentemente publicados sugerem mudanças em (1.28) (ver por exemplo, Gelfand e Dey, 1994). O uso das distribuições preditivas em alguma forma tem sido reconhecida como a correta abordagem Bayesiana para a seleção de modelos. Particularmente, Box (1980) argumenta que densidades a posteriori devem ser usadas para a estimação dos parâmetros condicional à adequabilidade do modelo, enquanto que densidades preditivas devem ser usadas para a análise da adequabilidade dos modelos na presença dos dados. Na avaliação de dois modelos quaisquer, as densidades preditivas podem ser comparadas enquanto que as densidades a posteriori nem sempre, conforme visto acima.

Outra alternativa para discriminação de modelos (Gelfand e Dey (1994) e Gelfand, Dey e Chang(1992)) é o uso da densidadle preditiva com validação cruzada dada por,

$$
f\left(t_{r} \mid D_{r}\right)=\frac{f(D)}{f\left(D_{(r)}\right)}=\int f\left(t_{r} \mid \beta, D_{(r)}\right) \pi\left(\beta \mid D_{(r)}\right) d \beta
$$


onde $D_{(r)}=\left(t_{1}, \ldots, t_{r-1}, t_{r+1}, \ldots, t_{n}\right)$ e $\pi\left(\beta \mid D_{(r)}\right)$ é a correspondente densidade a posteriori baseada em $D_{(r)}$. Essa densidade pode ser chamada de densidade preditiva condicional ordenada (CPO).

Este procedimento é rotineiramene usado em análise de regressão clássica e muitas técnicas de diagnósticos adotam esse ponto de vista. Isto também é estabelecido na literatura Bayesiana por Stone (1974).

Para obter uma estimativa da densidade preditiva de validação cruzada observe que

$$
\begin{aligned}
f\left(t_{r} \mid D_{r}\right) & =\frac{\int \frac{f(D \mid \beta) \pi(\beta)}{\pi(\beta \mid D) f(D)} \pi(\beta \mid D) d \beta}{\int \frac{f\left(D_{(r)} \mid \beta\right) \pi(\beta)}{\pi(\beta \mid D) f(D)} \pi(\beta \mid D) d \beta} \\
& =\frac{1}{\int \frac{1}{f\left(t_{r} \mid D_{r}, \beta\right)} \pi(\beta \mid D) d \beta} .
\end{aligned}
$$

Daí, por aproximação de Monte Carlo em (1.32) obtem-se a estimativa

$$
\hat{f}\left(t_{r} \mid D_{(r)}\right)=B\left(\sum_{i=1}^{B} \frac{1}{f\left(t_{r} \mid D_{(r)}, \beta_{\mathrm{i}}\right)}\right)^{-1}
$$

onde $B$ é o tamanho da amostra gerada da posteriori de $\beta$.

Se $t_{r}, r=1, \ldots, n$, são condicionalmente independentes dado $\beta, f\left(t_{r} \mid D_{(r)}, \beta_{\mathbf{i}}\right)$ pode ser simplificada para $f\left(t_{r} \mid \beta_{\mathbf{i}}\right)$. Observe que esta quantidade pode ser considerada como a r-ésima componente da função de verossimilhança ou como a densidade condicional de $t_{r}$ dado $\beta_{\mathrm{i}}$. Logo, (1.33) é a média harmônica das componentes da função verossimilhança ou média harmônica da densidade condicional avaliada na amostra a posteriori.

Podemos utilizar as estimativas $c_{r}(l)=\hat{f}\left(t_{r} \mid D_{(r)}\right)$ na seleção dos modelos, considerando um gráfico dos valores $c_{r}(l)$ versus o número de observações $r(r=1, \ldots, n)$ para diferentes modelos; valores maiores (em média) indica o melhor modelo. Também pode-se considerar para escolha do melhor modelo, escolher o modelo que maximiza $c(l)=\prod_{i=1}^{n} c_{r}(l)$, onde $l$ é o indexador do modelo.

Alternativamente, podemos considerar o gráfico da razão dos CPO's (ou log da razão (los CPO's) dos diferentes modelos contra o número de observações para selecionar o melhor modelo; valores da razão maiores que um (ou positivos) indicam a preferència do primeiro modelo.

Geisser e Eddy (1979) sugerem que o produto das densidades preditivas $\prod_{r=1}^{n} f\left(t_{r} \mid D_{(r)}, M_{l}\right)$ pode ser usada como um indicador na seleção de modelos. Se temos dois modelos $M_{1} \mathrm{e}$ 
$M_{2}$, temos a razão,

$$
\frac{\prod_{r=1}^{n} f\left(t_{r} \mid D_{(r)}, M_{l}\right)}{\prod_{r=1}^{n} f\left(t_{r} \mid D_{(r)}, M_{2}\right)}
$$

que pode ser usada como uma aproximação do fator de Bayes, a qual denota-se por $P S F B_{12}$ (pseudo-fator de Bayes) escolhendo-se o modelo $M_{1}$ se $P S F B_{12}>1$.

\subsection{Objetivos do Trabalho}

O processo de Poisson não-homogêneo é muito usado na modelagem do número de falhas em um sistemas de reparo e na confiabilidade de Software. Em geral, o uso de processos de Poisson não-homogêneos apresenta diferentes formas para a função intensidade que podem ser de formas crescentes ou decrescentes. Na prática, podemos ter superposição de dois ou mais processos de Poisson não-homogêneos e que leva à formas mais realistas da função intensidade. Podemos notar que a superposição de dois ou mais processos de Poisson não-homogêneos também é um processo de Poisson não-homogêneo (Kuo e Yang, 1996).

Em geral, é suposto que esses processos de Poisson não-homogêneos são independentes, uma suposição nem sempre verificada para os dados. Assim, vamos considerar neste trabalho a suposição de dependência dos PPNHs. Inferências sobre a superposição de dois processos de Poisson dependentes foram introduzido por Rodrigues, Ramires Cid e Achcar (2002). A hipótese de independência é conveniente sob o ponto de vista matemático, porém não é uma suposição que seja muito sustentável no mundo real. Daí a necessidade de considerar o problema da superposição no caso em que os processos componentes não são necessariamente independentes entre sí. Um exemplo real, na área médica, é dado em Aslanidou H. et al., (1998). Na área de modelagem de software, quando existe correlação entre dois processos de Poisson não-homogêneos para modelar dados de software, introduzimos a superposição de PPNHs bivariada, e introduzimos aspectos de inferência Bayesiana para estudar a superposição de dois PPNHs dependentes, usando métodos 
computacionais recentes para encontrar os sumários a posteriori de interesse (ver, por exemplo, Gelfand e Smith, 1990).

Uma outra questão importante discutida por pesquisadores na área médica diz respeito a inferência para mudança na taxa de falha para pacientes que recebem um dado tratamento. Aplicações em modelos com ponto de mudança são dados em muitas área de interesse. Por exemplo, pesquisadores médicos usualmente tem interesse em saber se um nova terapia de leucemia produz um distanciamento da experiência usual de uma taxa decorrida constante depois da indução de uma remissão (ver, por exemplo, Mathews e Farewell (1985)).

Dependendo do modelo paramétrico especificado para a variável aleatória T, denotando o tempo de vida do paciente, problemas de inferência podem ser de difícil solução considerando a abordagem clássica padrão. Diversos autores tem abordado problemas de ponto de mudança sob o ponto de vista Bayesiano (ver por exemplo Achcar e Bolfarine, 1989). Uma análise Bayesiana de um processo Poisson com um ponto de mudança é introduzido por Raftery e Akman (1986).

Raftery e Akman (1986) introduziram testes para existência de um ponto de mudança em processos de Poisson homogêneos (HPP) usando prioris informativas próprias para os parâmetros do modelo. Leonard (1978) propôs a estimação dos parâmetros do processo de Poisson com ponto de mudança minimizando a integral da diferença quadrática sob ligação paramétrica e não paramétrica. Problema similar foi discutido dentro de uma estrutura Bayesiana por Smith (1975), Kutoyants (1984), Kalbfleisch e Struthers (1982), Mathews and Farewell (1982) e Nguyen et al., (1984). Outros resultados inferenciais são obtidos por Muralidharan $(1999,2002)$.

Métodos Bayesianos tem sido usados por muitos autores para obter inferência para modelos com ponto de mudança (ver, por exemplo, Raftery e Akman 1986; Achcar e Bolfarine, 1989; Achcar e Loibel, 1998). Neste trabalho o problema de ponto de mudança é tratado sob a abordagem de processos de Poisson não-homogêneos usando o algoritmo MCMC.

Sob o enfoque Bayesiano, nas aplicações reais a escolha das distribuicões a priori pelos pesquisadores, geralmente incorporam conhecimentos de especialistas e informações técnicas dos engenheiros de software. Sob esse aspecto, Compodonico e Singpurwalla 
(1995), consideram a escolha das distribuições a priori para modelos baseados em PPNHs, sustentada nas informações de um especialista, por exemplo, relativas ao número de módulos, ao perfil operacional, etc. A partir desta informação consideram uma distribuição a priori conjunta para a função de valor médio, $m(t)$, para diferentes valores de t, e daí é possível tirar informações sobre os hiperparâmetros de interesse. Para discriminar os vários modelos propostos na análise de dados de confiabilidade de software, consideramos vários procedimentos Bayesianos entre os quais os critérios AIC, BIC e DIC (ver, por exemplo, Kass e Raftery (1995); Dey et al., (1999); Spiegelhalter et al., (2002)).

A seguir vamos descrever a forma que este trabalho de tese está organizado.

No Capítulo 2, consideramos superposição de um processo de Musa-Okumoto com um processo de Weibull independentes e dependentes na presença ou não de covariáveis.

No Capítulo 3 consideramos um processo de Poisson não-homogêneo (PPNH) com o modelo de uma função de intensidade polinomial, considerando a superposição de um processo de Poisson Homogêno e $J-1(J \geq 2)$ processos Weibull com parâmetro de forma conhecido.

No Capítulo 4 consideramos uma superposição de dois processo de lei de potência dependentes, que é um caso especial de um processo de Weibull com uma simples reparametrização ou conhecido como um modelo de crescimento em confiabilidade.

No capítulo 5 consideramos a existência de um ponto de mudança no PPNH; neste caso vamos obter inferências para o ponto de mudança no caso discreto e contínuo.

No capítulo 6 apresenta-se as conclusões e algumas propostas de continuidade deste trabalho de tese de doutorado. Um Apêndice foi introduzido com o objetivo de apresentar os métodos de diagnóstico de convergência para as cadeias geradas pelo método MCMC (Apêndice A) e a distribuição de Poisson Bivariada (Apêndice B).

Também apresentamos a geração de processos de Poisson e alguns programas desenvolvidos na implementação computacional para os exemplos de aplicação dos modelos propostos (ver Apêndice C e D). 


\section{Capítulo 2}

\section{Superposição de um Processo Musa e Okumoto com um Processo de Weibull na Presença de Covariáveis}

Apresentamos neste capítulo alguns modelos de superposição de um processo Musa e Okumoto com um processo de Weibull na presença de covariáveis. Damos alguns exemplos de aplicações dos conjuntos de dados simulados a partir do PPNH.

Nossa contribuição neste capítulo é a de introduzir uma análise Bayesiana para PPNH dependentes assumindo uma distribuição de Poisson bivariada (ver por exemplo, Ahmed 1981) que incorpora a dependência de subsistemas na presença de uma ou mais covariáveis. Inicialmente introduzimos um modelo genérico para a superposição de um processo de Musa e Okumoto com um processo de Weibull na presença de covariáveis.

\subsection{Descrição do Modelo Genérico}

O modelo definido aqui é um modelo de tempo de falha conforme (ii) dado em (1.14). Consideramos as funções intensidades componentes deste modelo assumindo um efeito multiplicativo de $p$ covariáveis, $x_{1 i}, x_{2 i}, \ldots, x_{p i}, i=1, \ldots, n$ dadas por

$$
\begin{aligned}
& \lambda_{1}\left(t_{i}\right)=\frac{\alpha_{1}}{t_{i}+a_{2}} \exp \left\{-\xi_{11} x_{1 i}-\xi_{12} x_{2 i}-\cdots-\xi_{1 p} x_{p i}\right\} \\
& \lambda_{2}\left(t_{i}\right)=3 t_{i} \exp \left\{-\xi_{21} x_{1 i}-\xi_{22} x_{2 i}-\cdots-\xi_{2 p} x_{p i}\right\} .
\end{aligned}
$$

Vamos assumir que além dos efeitos da covariávies nas funções intensidades $\lambda_{1}\left(t_{i}\right)$ e $\lambda_{2}\left(t_{i}\right)$ existe um efeito no parâmetro de correlação $\rho$ dado na forma aditiva 


$$
\rho=\gamma_{0}+\gamma_{1} x_{1 i}+\cdots+\gamma_{p} x_{p i}
$$

Modelo M1 (superposição de processos independentes sem a presença de covariáveis)

Considere o modelo de superposição do processo Musa e Okumoto com o processo de Weibull em (2.1) assumindo $p=0$, isto é, sem covariável as funções intensidades componentes deste modelo são dadas por,

$$
\lambda_{1}\left(t_{i}\right)=\frac{\alpha_{1}}{t_{i}+a_{2}} \quad \text { e } \quad \lambda_{2}\left(t_{i}\right)=\beta t_{i}
$$

A função do valor médio deste processo de superposição é dada por,

$$
m\left(t_{n}\right)=m_{1}\left(t_{n}\right)+m_{1}\left(t_{n}\right)=\alpha_{1} \ln \left(1+\frac{t_{n}}{\alpha_{2}}\right)+\frac{\beta t_{n}^{2}}{2} .
$$

Considerando as funções de intensidades (2.3) e a função de verossimilhança (1.18) $\operatorname{com} \rho=0$ é dada por,

$$
L\left(\underline{\beta} \mid \mathfrak{D}_{\mathfrak{t}}\right)=\exp \left\{-\left[\alpha_{1} \ln \left(1+\frac{t_{n}}{\alpha_{2}}\right)+\frac{\beta t_{n}^{2}}{2}\right]\right\} \prod_{i=1}^{n}\left[\frac{\alpha_{1}}{t_{i}+\alpha_{2}}+\beta t_{i}\right]
$$

Denotamos o vetor de parâmetros deste modelo por $\underline{\beta}=\left(\alpha_{1}, \alpha_{2}, \beta\right)$.

Uma análise Bayesiana deste modelo foi introduzida por Ramirez Cid et al. (1999).

Modelo M2 (superposição de processos dependentes na presença de uma covariável)

Considere o modelo de superposição do processo Musa e Okumoto com o processo de Weibull em (2.1) assumindo $p=1$, isto é, com a presença de uma covariável e as funções intensidades componentes deste modelo são dadas por,

$$
\lambda_{1}\left(t_{i}\right)=\frac{\alpha_{1}}{t_{i}+\alpha_{2}} e^{-\xi_{11} x_{1 i}} \text { e } \lambda_{2}\left(t_{i}\right)=\beta t_{i} e^{-\xi_{21} \cdot x_{1 i}}
$$

Neste caso, as funções de valor médio componentes $m_{1}\left(t_{n}\right)$ e $m_{2}\left(t_{n}\right)$ são dadas por

$$
m_{1}\left(t_{n}\right)=\alpha_{1} e^{-\xi_{11} \cdot x_{1 n}} \ln \left(1+\frac{t_{n}}{\alpha_{2}}\right) \quad \mathrm{e} m_{2}\left(t_{n}\right)=\frac{3 t_{n}^{2}}{2} e^{-\xi_{21} x_{1 n}} .
$$


A função intensidade correspondente é dada por

$$
\lambda\left(t_{i}\right)=\lambda_{1}\left(t_{i}\right)+\lambda_{2}\left(t_{i}\right)=\frac{\alpha_{1} e^{-\xi_{11} x_{1 i}}}{t_{i}+\alpha_{2}}+\beta t_{i} e^{-\xi_{21} x_{1 i}}
$$

Na Figura 2.1 temos o gráficos da função intensidade da superposição dos PPNH (2.8) com dados simulados, assumindo

dados1: $\quad \alpha_{1}=20, \alpha_{2}=100, \beta=0.0005, \xi_{1}=0,01$ e $\xi_{2}=65$

dados $2: \quad \alpha_{1}=15, \alpha_{2}=95, \beta=0.0045, \xi_{1}=0,03$ e $\xi_{2}=75$

dados3: $\alpha_{1}=20, \alpha_{2}=120, \beta=0.05, \xi_{1}=0,07$ e $\xi_{2}=85$

dados4: $\alpha_{1}=18, \alpha_{2}=150, \beta=0.00035, \xi_{1}=0,5 \mathrm{e} \xi_{2}=100$

dados5: $\alpha_{1}=30, \alpha_{2}=200, \beta=0.00035, \xi_{1}=0,03$ e $\xi_{2}=110$ com $x_{i}=0$ e 1 . Nessas situações, observamos que as taxas de falhas do modelo diminuem com o tempo.

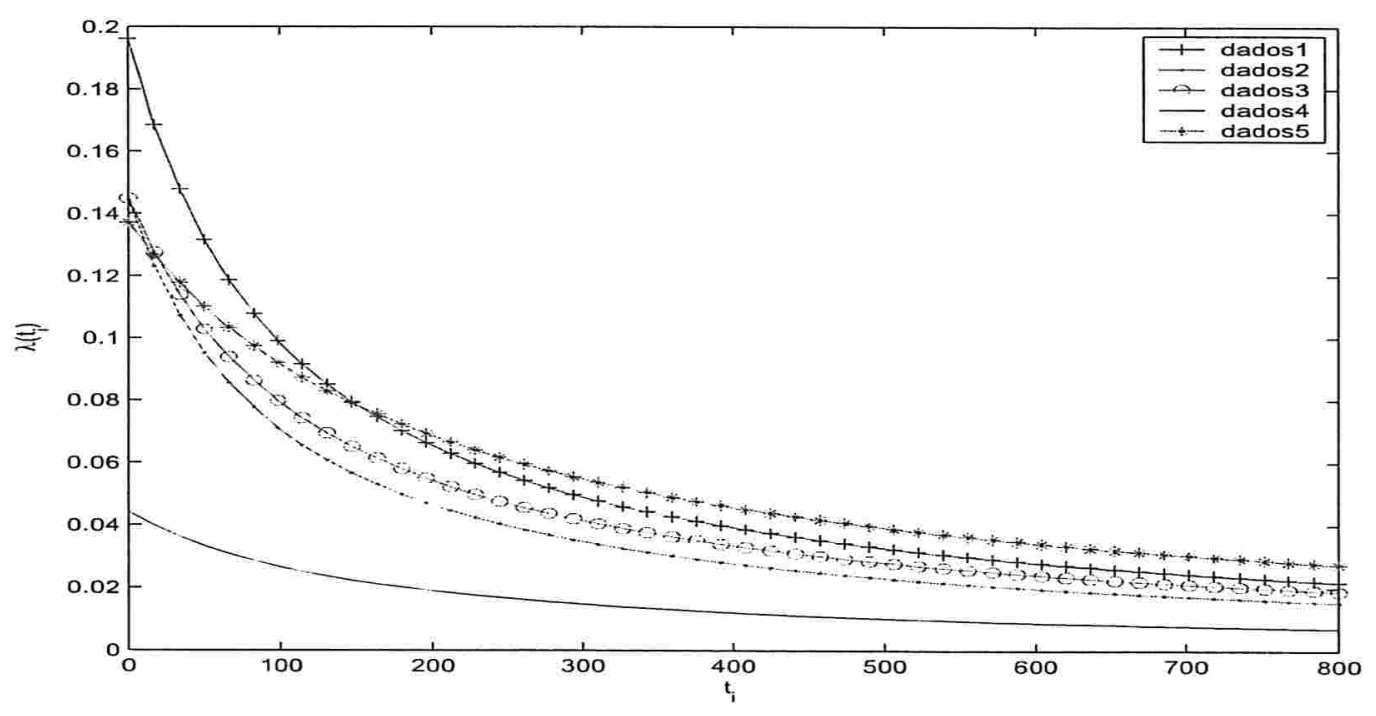

Figura 2.1: Função Intensidade

Vamos assumir que além do efeito da covariável $x_{1 i}$ nas funções intensidades $\lambda_{1}\left(t_{i}\right)$ e $\lambda_{2}\left(t_{i}\right)$ existe um efeito da covariável $x_{1 i}$ no parâmetro de correlação $\rho$, assumindo $p=1$ em (2.2). Assim, de (1.18) a função de verossimilhança é dada por

$$
\begin{aligned}
L\left(\underline{\beta} \mid \mathfrak{D}_{\mathrm{t}}\right) & =\prod_{i=1}^{n}\left[\lambda_{1}\left(t_{i}\right)+\lambda_{2}\left(t_{i}\right)\right]\left[1-\frac{\left(\gamma_{0}+\gamma_{1} x_{1 i}\right) W\left(t_{i}\right)}{2}\right] \\
& \times \exp \left\{-\left[m_{1}\left(t_{n}\right)+m_{2}\left(t_{n}\right)-\left(\gamma_{0}+\gamma_{1} x_{1 n}\right)\left(m_{1}\left(t_{n}\right) m_{2}\left(t_{n}\right)\right)^{1 / 2}\right]\right\}
\end{aligned}
$$


onde $W\left(t_{i}\right)$, é definido em (1.19), $m_{1}\left(t_{n}\right)$ e $m_{2}\left(t_{n}\right)$ em (2.7), respectivamente. O vetor de parâmetros deste modelo é dado por $\underline{\beta}=\left(\alpha_{1}, \alpha_{2}, \beta, \xi_{11}, \xi_{21}, \gamma_{0}, \gamma_{1}\right)$.

Modelo M3 (superposição de processos dependentes na presença de uma covariável)

Neste modelo vamos considerar o caso onde a covariável $x_{p i}$ não afeta o parâmetro de correlação $\rho$, isto é, consideramos $\rho$ constante.

Assumindo $p=1$ em (2.1) temos (2.6) as funções intensidades dos componentes, a função de verossimilhança (1.18) com $\rho$ constante é reescrita por,

$$
\begin{aligned}
L\left(\underline{\beta} \mid \mathfrak{D}_{\mathfrak{t}}\right) & =\prod_{i=1}^{n}\left[\frac{\alpha_{1}}{t_{i}+\alpha_{2}} e^{-\xi_{11} x_{1 i}}+\beta t_{i} e^{-\xi_{21} x_{1 i}}\right]\left[1-\frac{\rho W\left(t_{i}\right)}{2}\right] \\
& \times \exp \left\{-\left[m_{1}\left(t_{n}\right)+m_{2}\left(t_{n}\right)-\rho\left(m_{1}\left(t_{n}\right) m_{2}\left(t_{n}\right)\right)^{1 / 2}\right]\right\}
\end{aligned}
$$

onde $W\left(t_{i}\right)$ é definido em (1.19) e $m_{1}\left(t_{n}\right)$ e $m_{2}\left(t_{n}\right)$ em (2.7), respectivamente. Denotamos o vetor de parâmetros deste modelo por $\underline{\beta}=\left(\alpha_{1}, \alpha_{2}, \beta, \xi_{11}, \xi_{21}, \rho\right)$.

Modelo M4 (superposição de processos independentes na presença de uma covariável)

Considere o modelo de superposição do processo Musa e Okumoto com o processo de Weibull em (2.1), assumindo $p=1$ e $\rho=0$ temos a superposição de dois PPNH independentes. A função de verossimilhaça em (1.18) com $\rho=0$ é reescrita por,

$$
L\left(\underline{\beta} \mid \mathfrak{D}_{\mathrm{t}}\right)=\prod_{i=1}^{n}\left[\frac{\alpha_{1}}{t_{i}+\alpha_{2}} e^{-\xi_{11} x_{1 i}}+\beta t_{i} e^{-\xi_{21} x_{1 i}}\right] \exp \left\{-\left[m_{1}\left(t_{n}\right)+m_{2}\left(t_{n}\right)\right]\right\} .
$$

Denotamos o vetor de parâmetros deste modelo por $\underline{\beta}=\left(\alpha_{1}, \alpha_{2}, \beta, \xi_{11}, \xi_{21}\right)$.

Uma análise Bayesiana deste modelo foi introduzida (ver Ramirez, Cid et al., 2001).

Modelo M5 (superposição de dois processos dependentes na presença de duas covariáveis)

Considere o modelo de superposição do processo de Musa e Okumoto com o processo de Weibull em (2.1), assumindo $p=2$, isto é, na presença de duas covariáveis as funções intensidades componentes deste modelo são cladas por,

$$
\lambda_{1}\left(t_{i}\right)=\frac{\alpha_{1}}{t_{i}+\alpha_{2}} e^{-\xi_{11} x_{1 i}-\xi_{12} x_{2 i}} \text { e } \lambda_{2}\left(t_{i}\right)=\beta t_{i} e^{-\xi_{21} x_{1 i}-\xi_{22} x_{2 i}}
$$


Neste caso, as funções de valor médio $m_{1}\left(t_{n}\right)$ e $m_{2}\left(t_{n}\right)$ são dadas por

$$
m\left(t_{n}\right)=m_{1}\left(t_{n}\right)+m_{2}\left(t_{n}\right)=\alpha_{1} e^{-\xi_{11} x_{1 n}-\xi_{12} x_{2 n}} \ln \left(1+\frac{t_{n}}{\alpha_{2}}\right)+\frac{\beta t_{n}^{2}}{2} e^{-\xi_{21} x_{1 n}-\xi_{22} x_{2 n}}
$$

Assim, a função intensidade é dada por

$$
\lambda\left(t_{i}\right)=\lambda_{1}\left(t_{i}\right)+\lambda_{2}\left(t_{i}\right)=\frac{\alpha_{1}}{t_{i}+\alpha_{2}} e^{-\xi_{11} x_{1 i}-\xi_{12} x_{2 i}}+\beta t_{i} e^{-\xi_{21} x_{1 i}-\xi_{22} x_{2 i}}
$$

Vamos assumir que além dos efeitos das covariáveis $x_{1 i}$ e $x_{2 i}$ nas funções intensidades $\lambda_{1}\left(t_{i}\right)$ e $\lambda_{2}\left(t_{i}\right)$ existe um efeito no parâmetro de correlação $\rho$, asssumindo $p=2$ em (2.2) o parâmetro de correlação $\rho$ é dado por:

$$
\rho=\gamma_{0}+\gamma_{1} x_{1 i}+\gamma_{2} x_{2 i}
$$

Assim, de (1.18) a função de verossimilhança é dada por

$$
\begin{aligned}
L\left(\underline{\beta} \mid \mathfrak{D}_{\mathrm{t}}\right)= & \prod_{i=1}^{n}\left[\lambda_{1}\left(t_{i}\right)+\lambda_{2}\left(t_{i}\right)\right]\left[1-\frac{\left(\gamma_{0}+\gamma_{1} x_{1 i}+\gamma_{2} x_{2 i}\right) W\left(t_{i}\right)}{2}\right] \exp \left\{-\left[m_{1}\left(t_{n}\right)+\right.\right. \\
& \left.\left.m_{2}\left(t_{n}\right)-\left(\gamma_{0}+\gamma_{1} x_{1 n}+\gamma_{2} x_{2 n}\right)\left(m_{1}\left(t_{n}\right) m_{2}\left(t_{n}\right)\right)^{1 / 2}\right]\right\}
\end{aligned}
$$

onde $W\left(t_{i}\right)$ é definido em (1.19) e $m_{1}\left(t_{n}\right)$ e $m_{2}\left(t_{n}\right)$ em (2.12) e o vetor de parâmetros deste modelo é dado por: $\underline{\beta}=\left(\alpha_{1}, \alpha_{2}, \beta, \xi_{11}, \xi_{12}, \xi_{21}, \xi_{22}, \gamma_{0}, \gamma_{1}, \gamma_{2}\right)$.

\section{Modelo M6 (superposição de dois processos dependentes)}

Neste modelo vamos considerar o caso onde as covariáveis $x_{1 i}$ e $x_{2 i}$ não afetam o parâmetro de correlação $\rho$ dado em (2.2), isto é, com $\rho$ constante.

Assumindo $p=2$ em (2.1) temos a função intensidade deste modelo dado em (2.13), a função de verossimilhança (1.18) com $\rho$ constante é reescrita por:

$$
\begin{aligned}
L\left(\underline{\beta} \mid \mathfrak{D}_{\mathfrak{t}}\right) & =\prod_{i=1}^{n}\left[\frac{\alpha_{1} e^{-\xi_{1} x_{1 i}-\nu_{1} x_{2 i}}}{t_{i}+\alpha_{2}}+\beta t_{i} e^{-\xi_{2} \cdot x_{1 i}-\nu_{2} \cdot x_{2 i}}\right] \cdot\left[1-\frac{\rho W\left(t_{i}\right)}{2}\right] \\
& \times \exp \left\{-\left[m_{1}\left(t_{n}\right)+m_{2}\left(t_{n}\right)-\rho\left(m_{1}\left(t_{n}\right) m_{2}\left(t_{n}\right)\right)^{1 / 2}\right]\right\}
\end{aligned}
$$

onde $W\left(t_{i}\right)$ é definido em (1.19) e $m_{1}\left(t_{n}\right)$ e $m_{2}\left(t_{n}\right)$ em (2.12). Denotamos o vetor de parâmetros deste modelo por $\underline{\beta}=\left(\alpha_{1}, \alpha_{2}, \beta, \xi_{11}, \xi_{12}, \xi_{21}, \xi_{22}, \rho\right)$. 
Modelo M7 (superposição de dois processos independentes na presença de duas covariáveis)

Neste modelo assumimos a superposição do processo de Musa Okumoto com o processo de Weibull independentes, isto é, consideramos $\rho=0$ e a presença das covariáveis $x_{1 i}$ e $x_{2 i}$ afetando a função intensidade dada (2.13). A função de verossimilhança (1.18) com $\rho=0$ é reescrita por:

$$
\begin{aligned}
L\left(\underline{\beta} \mid \mathfrak{D}_{\mathfrak{t}}\right) & =\prod_{i=1}^{n}\left[\frac{\alpha_{1}}{t_{i}+\alpha_{2}} e^{-\xi_{11} x_{1 i}-\xi_{12} x_{2 i}}+\beta t_{i} e^{-\xi_{21} x_{1 i}-\xi_{22} x_{2 i}}\right] \\
& \times \exp \left\{-\left[m_{1}\left(t_{n}\right)+m_{2}\left(t_{n}\right)\right]\right\} .
\end{aligned}
$$

Denotamos o vetor de parâmetros deste modelo por $\underline{\beta}=\left(\alpha_{1}, \alpha_{2}, \beta, \xi_{11}, \xi_{12}, \xi_{21}, \xi_{22}\right)$.

\subsection{Uma Análise Bayesiana para a Superposição de um Processo Musa Okumoto com um Processo Weibull}

Seguindo o procedimento padrão de inferência Bayesiana dada na seção (1.3) e considerando a superposição de dois processo PPNH dependentes $(j=1,2)$ e assumindo independência a priori entre $\underline{\beta}$ e $\rho$, temos uma distribuição a priori conjunta para $\underline{\beta}$ e $\rho$ dada por:

$$
\pi(\underline{\beta}, \rho)=\pi(\rho) \pi(\underline{\beta})
$$

De (1.18)-(2.18) e do teorema de Bayes podemos mostrar que a densidade a posteriori conjunta para $\underline{\beta}$ e $\rho$ é dada por:

$$
\begin{aligned}
\pi\left(\rho, \underline{\beta} \mid \mathfrak{D}_{\mathrm{t}}\right) \propto & \prod_{i=1}^{n}\left[\lambda_{1}\left(t_{i}\right)+\lambda_{2}\left(t_{i}\right)\right]\left(1-\frac{\rho W\left(t_{i}\right)}{2}\right) \exp \left\{-\left[m_{1}\left(t_{n}\right)+m_{2}\left(t_{n}\right)-\right.\right. \\
& \left.\left.\rho\left(m_{1}\left(t_{n}\right) m_{2}\left(t_{n}\right)\right)^{1 / 2}\right]\right\} \pi_{1}(\rho) \pi_{2}(\underline{\beta})
\end{aligned}
$$

onde $0 \leq \rho \leq 1$ e $\mathfrak{D}_{\mathfrak{t}}$ denota o conjunto de observações.

Notamos que a densidacle a posteriori conjunta (2.19) não é uma densidade padrão conhecida, portanto só podemos obter sumários a posteriori de interesse por meio de métodos de aproximação, tais como o método de Laplace (Tierney, Kass e Kadane,1986) 
ou usando métodos de simulação de Monte Carlo via Cadeias de Markov, tais como Gibbs Sampling (Casela e George, 1992) e Metropolis-Hastings (Chib e Greenberg, 1995).

Para simplificar as distribuições condicionais necessárias para o algoritmo Gibbs Sampling aplicamos a técnica de Data augmentation (veja Tanner e Wong, 1987), mediante a inclusão de variáveis latentes. Procedendo desta forma, a densidade conjunta dos dados e das variáveis latentes tem componentes independentes e identicamente distribuidas (ver por exemplo, Kuo e Peng, 1999).

De forma mais especifica, vamos gerar uma variável latente $\underline{v}_{i}=\left(v_{i 1}, v_{i 2}\right), \quad v_{i 1}+v_{i 2}=$ $1, \quad i=1, \ldots, n$ onde $v_{i 1} \sim \operatorname{Bernoulli}\left(h_{i 1}\right)$, e a probabilidade de sucesso $h_{i 1}\left(\right.$ para $\left.v_{i 1}=1\right)$ é dada por:

$$
h_{i 1}=\frac{\lambda_{1}\left(t_{i}\right)\left[1-\frac{\rho}{2}\left(\frac{m_{2}\left(t_{i}\right)}{m_{1}\left(t_{i}\right)}\right)^{1 / 2}\right]}{\lambda_{1}\left(t_{i}\right)\left[1-\frac{\rho}{2}\left(\frac{m_{2}\left(t_{i}\right)}{m_{1}\left(t_{i}\right)}\right)^{1 / 2}\right]+\lambda_{2}\left(t_{i}\right)\left[1-\frac{\rho}{2}\left(\frac{m_{1}\left(t_{i}\right)}{m_{2}\left(t_{i}\right)}\right)^{1 / 2}\right]} .
$$

Portanto, a distribuição a posteriori conjunta para $\underline{\beta}$ e $\rho$ considerando a introdução da variável latente $\underline{v}$ é dada por:

$$
\begin{aligned}
\pi\left(\underline{\beta}, \rho \mid \mathfrak{D}_{\mathrm{t}}, \underline{v}\right) \propto & \prod_{i=1}^{n} \lambda_{1}^{v_{i 1}}\left(t_{i}\right)\left[1-\frac{\rho}{2}\left(\frac{m_{2}\left(t_{i}\right)}{m_{1}\left(t_{i}\right)}\right)^{1 / 2}\right]^{v_{i 1}} \times \lambda_{2}^{v_{i 2}}\left(t_{i}\right)\left[1-\frac{\rho}{2}\left(\frac{m_{1}\left(t_{i}\right)}{m_{2}\left(t_{i}\right)}\right)^{1 / 2}\right]^{v_{i 2}} \times \\
& \pi_{1}(\rho) \pi_{2}(\underline{\beta}) \times \exp \left\{-\left[m_{1}\left(t_{n}\right)+m_{2}\left(t_{n}\right)-\rho\left(m_{1}\left(t_{n}\right) m_{2}\left(t_{n}\right)\right)^{1 / 2}\right]\right\}(2.21)
\end{aligned}
$$

Para obter amostras da distribuição a posteriori conjunta (2.21), seguimos o seguinte algoritmo:

Dado $\underline{\beta}^{(0)}$ um valor inicial,

(i) gerar $\underline{\nu_{1}}, \ldots, \underline{\nu_{n}}$ distribuição de $\operatorname{Bernoulli}\left(h_{i 1}\right), i=1, \ldots, n$; onde $h_{i 1}$ é dado em $(2.20)$

(ii) gerar $\underline{\beta_{j}}$ das distribuições condicionais $\pi\left(\underline{\beta_{j}} \mid \underline{\theta}_{\left(\underline{\beta_{j}}\right)}, \underline{\nu}, \underline{t}\right)$ onde $\underline{\theta}_{\left(\underline{\beta_{j}}\right)}$ denota o vetor de todos os parâmetros exceto $\underline{\beta_{j}}$.

A seguir introduzimos uma análise Bayesiana para os modelos descritos anteriormente. 


\subsubsection{Análise Bayesiana para o Modelo M1 (superposição de processos independentes sem a presença de covariáveis)}

Para uma análise Bayesiana desse modelo, consideramos as seguintes distribuições a priori para $\alpha_{1}, \alpha_{2}$ e $\beta$ :

$$
\begin{aligned}
\alpha_{1} & \sim \Gamma\left(a_{1}, b_{1}\right) ; \operatorname{com} a_{1}, b_{1} \text { conhecidas } \\
\alpha_{2} & \sim \Gamma\left(a_{2}, b_{2}\right) ; \operatorname{com} a_{2}, b_{2} \text { conhecidas } \\
\beta & \sim \Gamma\left(a_{3}, b_{3}\right) ; \text { com } a_{3}, b_{3} \text { conhecidas }
\end{aligned}
$$

onde, $\Gamma(a, b)$ denota uma distribuição gama com média $a / b$ e variância $a / b^{2}$. Também assumimos independência a priori entre os parâmetros $\alpha_{1}, \alpha_{2}$ e $\beta$.

Existem muitas possibilidades para a escolha de distribuição priori. A escolha da distribuição foi feita de acordo com o domínio do espaço paramétrico, isto é, gama para os parâmetros positivos $\left(\alpha_{1}, \alpha_{2}, \beta\right)$. Uma pequena análise de sensitividade foi feita para escolher os diferentes valores dos hiperparâmetros, os resultados estão mostrado nas tabelas dos resumos a posteriori para os modelos, onde observamos que as diferentes escolhas não modificam substancialmente os resultados apresentados ( aplicações dadas na seção 2.3 exemplo 1).

Neste caso, da distribuição a posteriori conjunta dada em (2.21) observamos que as densidades a posteriori condicionais para a aplicação do algoritmo de Gibbs são dadas por

(a) $\pi\left(\alpha_{1} \mid \underline{\theta}_{\left(\alpha_{1}\right)}, \underline{\nu}, \mathfrak{D}_{t}\right) \propto \alpha_{1}^{a_{1}-1} e^{-b_{1} \alpha_{1}} \psi_{1}(\underline{\beta})$

onde:

$$
\psi_{1}(\underline{\beta})=\exp \left\{\left(\sum_{i=1}^{n} \nu_{i 1}\right) \ln \left(\alpha_{1}\right)-\alpha_{1} \ln \left(1+\frac{t_{n}}{\alpha_{2}}\right)\right\}
$$

(b) $\pi\left(\alpha_{2} \mid \underline{\theta}_{\left(\alpha_{2}\right)}, \underline{\nu}, \mathfrak{D}_{\mathfrak{t}}\right) \propto \alpha_{2}^{a_{2}-1} e^{-b_{2} \alpha_{1}} \psi_{2}(\underline{\beta})$

onde:

$$
\nu_{2}(\underline{\beta})=\exp \left\{-\sum_{i=1}^{n} \nu_{i 1} \ln \left(\alpha_{2}+t_{i}\right)-\alpha_{1} \ln \left(1+\frac{t_{n}}{\alpha_{2}}\right)\right\}
$$


(c) $\pi\left(\beta \mid \underline{\theta}_{(\beta)}, \underline{\nu}, \mathfrak{D}_{\mathfrak{t}}\right) \propto \beta^{a_{3}-1} e^{-b_{3} \beta} \psi_{3}(\underline{\beta})$

onde:

$$
\psi_{3}(\underline{\beta})=\exp \left\{\left(\sum_{i=1}^{n} \nu_{i 2}\right) \ln (\beta)-\frac{\beta t_{n}^{2}}{2}\right\} .
$$

As distribuições condicionais a posteriori dadas em (2.23) não possuem uma distribuição padrão conhecida, de modo que faremos uso do algoritmo de Metropolis-Hastings (ver, Chib e Greenberg, 1995) para gerar amostras a posteriori de $\alpha_{1}, \alpha_{2}$ e $\beta$.

\subsubsection{Análise Bayesiana para o Modelo M2 (superposição de processos dependentes na presença de um covariável)}

Assumimos as seguintes distribuições a priori para $\alpha_{1}, \alpha_{2}, \beta, \xi_{11}, \xi_{21}, \gamma_{0}$ e $\gamma_{1}$ :

$$
\begin{aligned}
\alpha_{1} & \sim \Gamma\left(a_{1}, b_{1}\right) ; \operatorname{com} a_{1}, b_{1} \text { conhecidas } \\
\alpha_{2} & \sim \Gamma\left(a_{2}, b_{2}\right) ; \operatorname{com} a_{2}, b_{2} \text { conhecidas } \\
\beta & \sim \Gamma\left(a_{3}, b_{3}\right) ; \operatorname{com} a_{3}, b_{3} \text { conhecidas } \\
\xi_{11} & \sim N\left(c_{1}, d_{1}\right) ; \operatorname{com} c_{1}, d_{1} \text { conhecidas } \\
\xi_{21} & \sim N\left(c_{2}, d_{2}\right) ; \operatorname{com} c_{2}, d_{2} \text { conhecidas } \\
\gamma_{0} & \sim B\left(e_{1}, f_{1}\right) ; \operatorname{com} e_{1}, f_{1} \text { conhecidas } \\
\gamma_{1} & \sim B\left(e_{2}, f_{2}\right) ; \operatorname{com} e_{2}, f_{2} \text { conhecidas }
\end{aligned}
$$

onde, distribuição gama para os parâmetros positivos $\left(\alpha_{1}, \alpha_{2}, 3\right), N\left(\mu, \sigma^{2}\right)$ denota uma distribuição normal com média $\mu$ e variância $\sigma^{2}$ e $B(e, f)$ denota uma distribuição Beta com média $\frac{e}{e+f}$ e variância $\frac{e f}{(e+f)^{2}(e+f+1)}$. Também assumimos independência a priori entre os parâmetros $\alpha_{1}, \alpha_{2}, \beta, \xi_{11}, \xi_{21}, \gamma_{0}$ e $\gamma_{1}$.

Observamos que a correlação $\rho\left(\rho\left(x_{i}\right)=\gamma_{0}+\gamma_{1} x_{i}, i=1, \cdots, n\right)$ é definida no intervalo $(0,1)$, isto é, $0 \leq \rho \leq 1$. A escolha das distribuições a priori Beta para $\gamma_{0}$ e $\gamma_{1}$ devem levar em consideração essa restrição existente, isto é, $0 \leq \gamma_{0}+\gamma_{11} x_{i} \leq 1$.

Existem muitas possibilidades para a escolha de distribuição priori. Neste trabalho a escolha da distribuição foi feita de acordo com o domínio do espaço paramétrico, isto é, 
distribuição gama para os parâmetros positivos, normal para os parâmetros definidos na reta $\left(\xi_{11}, \xi_{21}\right)$ e distribuição beta para os parâmetros $\left(\gamma_{0}, \gamma_{1}\right)$ que estão variando entre 0 e 1. Uma pequena análise de sensitividade foi feita para escolher os diferentes valores dos hiperparâmetros; os resultados são dados nas Tabelas dos resumos a posteriori para os modelos, onde observamos que as diferentes escolhas não modificam substancialmente os resultados apresentados ( aplicações dadas na seção 2.3 , exemplo 1).

Neste caso, as densidades a posteriori condicionais necessárias para o algoritmo Gibbs Sampling são dadas por:

(a) $\pi\left(\alpha_{1} \mid \underline{\theta}_{\left(\alpha_{1}\right)}, \underline{\nu}, \mathfrak{D}_{\mathfrak{t}}\right) \propto \alpha_{1}^{a_{1}-1} e^{-b_{1} \alpha_{1}} \psi_{1}(\underline{\beta})$

onde:

$$
\begin{aligned}
\psi_{1}(\underline{\beta}) & =\exp \left\{\left(\sum_{i=1}^{n} \nu_{i 1}\right) \ln \left(\alpha_{1}\right)+\sum_{i=1}^{n} \nu_{i 1} \ln \left(1-\frac{\rho\left(x_{1 i}\right) A_{1 i}(\underline{\beta})}{2}\right)+\sum_{i=1}^{n} \nu_{i 2} \ln \left(1-\frac{\rho\left(x_{1 i}\right) A_{2 i}(\underline{\beta})}{2}\right)\right. \\
& \left.-\alpha_{1} e^{-\xi_{11} x_{1 n}} \ln \left(1+\frac{t_{n}}{\alpha_{2}}\right)+\rho\left(x_{1 i}\right)\left[\frac{\alpha_{1} \beta}{2} t_{n}^{2} e^{-\left(\xi_{11}+\xi_{21}\right) x_{1 n}} \ln \left(1+\frac{t_{n}}{\alpha_{2}}\right)\right]^{1 / 2}\right\} .
\end{aligned}
$$

Para simplificar denotamos

$$
A_{1 i}\left(\alpha_{1}, \alpha_{2}, \beta, \xi_{11}, \xi_{21}\right) \text { e } A_{2 i}\left(\alpha_{1}, \alpha_{2}, \beta, \xi_{11}, \xi_{21}\right)
$$

por $A_{1 i}(\underline{\beta})$ e $A_{2 i}(\underline{\beta})$, onde,

(i) $A_{1 i}(\underline{\beta})=\left[\frac{m_{2}\left(t_{i}\right)}{m_{1}\left(t_{i}\right)}\right]^{1 / 2}=\frac{\beta^{1 / 2} t_{i} e^{\frac{-\xi_{21} \cdot r_{1 n}}{2}}}{\sqrt{2 \alpha_{1} e^{-\xi_{11} x_{1 n}} \ln \left(1+\frac{t_{i}}{\alpha_{2}}\right)}}$,

(ii) $A_{2 i}(\underline{\beta})=\left[\frac{m_{1}\left(t_{i}\right)}{m_{2}\left(t_{i}\right)}\right]^{1 / 2}=\frac{\sqrt{2 \alpha_{1} e^{-\xi_{11} x_{1 n} \ln \left(1+\frac{t_{i}}{\beta}\right)}}}{3^{1 / 2} t_{i} e^{-\frac{\xi_{21} x_{1 n}}{2}}}$

(b) $\pi\left(\alpha_{2} \mid \underline{\theta}_{\left(\alpha_{2}\right)}, \underline{\nu}, \mathfrak{D}_{\mathrm{t}}\right) \propto \alpha_{2}^{a_{2}-1} e^{-b_{2} \alpha_{1}} \psi_{2}(\underline{\beta})$

onde:

$$
\begin{aligned}
\psi_{2}(\underline{\beta})= & \exp \left\{-\sum_{i=1}^{n} \nu_{i 1} \ln \left(\alpha_{2}+t_{i}\right)+\sum_{i=1}^{n} \nu_{i 1} \ln \left(1-\frac{\rho\left(x_{1 i}\right) A_{1 i}(\underline{3})}{2}\right)+\sum_{i=1}^{n} \nu_{i 2} \times\right. \\
& \ln \left(1-\frac{\rho\left(x_{1 i}\right) A_{2 i}(\underline{3})}{2}\right)-\alpha_{1} e^{-\xi_{11} \cdot x_{1 n}} \ln \left(1+\frac{t_{n}}{\alpha_{2}}\right)+\rho\left(x_{1 i}\right)\left[\frac{\alpha_{1} \beta}{2} t_{n}^{2} e^{-\left(\xi_{11}+\xi_{21}\right) \cdot x_{1 n}} \times\right. \\
& \left.\left.\ln \left(1+\frac{t_{n}}{\alpha_{2}}\right)\right]^{1 / 2}\right\}
\end{aligned}
$$


(c) $\pi\left(\beta \mid \underline{\theta}_{(\beta)}, \underline{\nu}, \mathfrak{D}_{\mathfrak{t}}\right) \propto \beta^{a_{3}-1} e^{-b_{3} \beta} \psi_{3}(\underline{\beta})$

onde:

$$
\begin{aligned}
& \psi_{3}(\underline{\beta})=\exp \left\{\left(\sum_{i=1}^{n} \nu_{i 2}\right) \ln (\beta)+\sum_{i=1}^{n} \nu_{i 1} \ln \left(1-\frac{\rho\left(x_{1 i}\right) A_{1 i}(\underline{\beta})}{2}\right)+\sum_{i=1}^{n} \nu_{i 2} \ln \left(1-\frac{\rho\left(x_{1 i}\right) A_{2 i}(\underline{\beta})}{2}\right)\right. \\
&\left.-\frac{\beta}{2} t_{n}^{2} e^{-\xi_{21} x_{1 n}}+\rho\left(x_{1 i}\right)\left[\frac{\alpha_{1} \beta}{2} t_{n}^{2} e^{-\left(\xi_{11}+\xi_{21}\right) x_{1 n}} \ln \left(1+\frac{t_{n}}{\alpha_{2}}\right)\right]^{1 / 2}\right\} \\
& \text { (d) } \pi\left(\xi_{1} \mid \underline{\theta}_{\left(\xi_{1}\right)}, \underline{\nu}, \mathfrak{D}_{\mathrm{t}}\right) \propto \exp \left\{-\frac{1}{2 d_{1}^{2}}\left(\xi_{1}-c_{1}\right)^{2}\right\} \psi_{4}(\underline{\beta})
\end{aligned}
$$

onde:

$$
\begin{aligned}
& \psi_{4}(\underline{\beta})=\exp \left\{-\left(\sum_{i=1}^{n} \nu_{i 1} x_{1 i}\right) \xi_{11}+\sum_{i=1}^{n} \nu_{i 1} \ln \left(1-\frac{\rho\left(x_{1 i}\right) A_{1 i}(\underline{\beta})}{2}\right)+\sum_{i=1}^{n} \nu_{i 2} \ln \left(1-\frac{\rho\left(x_{1 i}\right) A_{2 i}(\underline{\beta})}{2}\right)\right. \\
&\left.-\alpha_{1} e^{-\xi_{11} x_{1 n}} \ln \left(1+\frac{t_{n}}{\alpha_{2}}\right)+\rho\left(x_{1 i}\right)\left[\frac{\alpha_{1} \beta}{2} t_{n}^{2} e^{-\left(\xi_{11}+\xi_{21}\right) x_{1 n}} \ln \left(1+\frac{t_{n}}{\alpha_{2}}\right)\right]^{1 / 2}\right\} \\
& \text { (e) } \pi\left(\xi_{21} \mid \underline{\theta}_{\left(\xi_{21}\right)}, \underline{\nu}, \mathfrak{D}_{\mathrm{t}}\right) \propto \exp \left\{-\frac{1}{2 d_{2}^{2}}\left(\xi_{21}-c_{2}\right)^{2}\right\} \psi_{5}(\underline{\beta})
\end{aligned}
$$

onde:

$$
\begin{aligned}
& \psi_{5}(\underline{\beta})=\exp \left\{\sum_{i=1}^{n} \nu_{i 1} \ln \left(1-\frac{\rho\left(x_{1 i}\right) A_{1 i}(\underline{\beta})}{2}\right)-\left(\sum_{i=1}^{n} \nu_{i 2} x_{1 i}\right) \xi_{21}+\sum_{i=1}^{n} \nu_{i 2} \ln \left(1-\frac{\rho\left(x_{1 i}\right) A_{2 i}(\underline{\beta})}{2}\right)\right. \\
&\left.-\frac{\beta t_{n}^{2}}{2} e^{-\xi_{21} x_{1 n}}+\rho\left(x_{1 i}\right)\left[\frac{\alpha_{1} \beta}{2} t_{n}^{2} e^{-\left(\xi_{11}+\xi_{21}\right) x_{1 n}} \ln \left(1+\frac{t_{n}}{\alpha_{2}}\right)\right]^{1 / 2}\right\} \\
& \text { (f) } \pi\left(\gamma_{0} \mid \underline{\theta}_{\left(\gamma_{0}\right)}, \underline{\nu}, \mathfrak{D}_{\mathfrak{t}}\right) \propto \gamma_{0}^{c_{1}-1}\left(1-\gamma_{0}\right)^{d_{1}-1} \psi_{6}(\underline{\beta})
\end{aligned}
$$

onde:

$$
\begin{aligned}
\psi_{6}(\underline{\beta}) & =\exp \left\{\sum_{i=1}^{n} \nu_{i 1} \ln \left(1-\frac{\rho\left(x_{1 i}\right) A_{1 i}(\underline{\beta})}{2}\right)+\sum_{i=1}^{n} \nu_{i 2} \ln \left(1-\frac{\rho\left(x_{1 i}\right) A_{2 i}(\underline{\beta})}{2}\right)\right. \\
& \left.+\rho\left(x_{1 i}\right)\left[\frac{\alpha_{1} \beta}{2} t_{n}^{2} e^{-\left(\xi_{11}+\xi_{21}\right) x_{1 n}} \ln \left(1+\frac{t_{n}}{\alpha_{2}}\right)\right]^{1 / 2}\right\}
\end{aligned}
$$

(g) $\pi\left(\gamma_{1} \mid \underline{\theta}_{\left(\gamma_{1}\right)}, \underline{\nu}, \mathfrak{D}_{\mathrm{t}}\right) \propto \gamma_{1}^{c_{2}-1}\left(1-\gamma_{1}\right)^{d_{2}-1} \psi_{7}(\underline{\beta})$

onde:

$$
\begin{aligned}
\dot{\psi}_{7}(\underline{\beta}) & =\exp \left\{\sum_{i=1}^{n} \nu_{i 1} \ln \left(1-\frac{\rho\left(x_{1 i}\right) A_{1 i}(\underline{\beta})}{2}\right)+\sum_{i=1}^{n} \nu_{i 2} \ln \left(1-\frac{\rho\left(x_{1 i}\right) A_{2 i}(\underline{\beta})}{2}\right)\right. \\
& \left.+\rho\left(x_{1 i}\right)\left[\frac{\alpha_{1} \beta}{2} t_{n}^{2} e^{-\left(\xi_{11}+\xi_{21}\right) \cdot x_{1 n}} \ln \left(1+\frac{t_{n}}{\alpha_{2}}\right)\right]^{1 / 2}\right\}
\end{aligned}
$$

As distribuições condicionais a posteriori dadas em (2.25) não apresentam uma distribuição padrão conhecida, de modo que faremos uso do algoritmo de Metropolis-Hastings (Ver. Chib e Greenberg, 1995) para gerar amostras a posteriori dos parâmetros $\alpha_{1}, \alpha_{2}, 3$, $\xi_{11}, \xi_{21}, \gamma_{0}$ e $\gamma_{1}$ 


\subsubsection{Análise Bayesiana para o Modelo M3 (superposição de processos dependentes na presença de uma covariável)}

Assumimos as seguintes distribuições a priori para $\alpha_{1}, \alpha_{2}, \beta, \xi_{11}, \xi_{21}$ e $\rho$ :

$$
\begin{aligned}
\alpha_{1} & \sim \Gamma\left(a_{1}, b_{1}\right) ; \operatorname{com} a_{1}, b_{1} \text { conhecidas } \\
\alpha_{2} & \sim \Gamma\left(a_{2}, b_{2}\right) ; \operatorname{com} a_{2}, b_{2} \text { conhecidas } \\
\beta & \sim \Gamma\left(a_{3}, b_{3}\right) ; \operatorname{com} a_{3}, b_{3} \text { conhecidas } \\
\xi_{11} & \sim N\left(c_{1}, d_{1}\right) ; \operatorname{com} c_{1}, d_{1} \text { conhecidas } \\
\xi_{21} & \sim N\left(c_{2}, d_{2}\right) ; \operatorname{com} c_{2}, d_{2} \text { conhecidas } \\
\rho & \sim B\left(e_{1}, f_{1}\right) ; \operatorname{com} e_{1}, f_{1} \text { conhecidas }
\end{aligned}
$$

Da distribuição a posteriori conjunta (2.21) pode-se mostrar que as densidades a posteriori condicionais para a aplicação do algoritmo de Gibbs são dados por:

(a) $\pi\left(\alpha_{1} \mid \underline{\theta}_{\left(\alpha_{1}\right)}, \underline{\nu}, \mathfrak{D}_{\mathfrak{t}}\right) \propto \alpha_{1}^{a_{1}-1} e^{-b_{1} \alpha_{1}} \psi_{1}(\underline{\beta})$

onde:

$$
\begin{aligned}
\psi_{1}(\underline{\beta}) & =\exp \left\{\left(\sum_{i=1}^{n} \nu_{i 1}\right) \ln \left(\alpha_{1}\right)+\sum_{i=1}^{n} \nu_{i 1} \ln \left(1-\frac{\rho A_{1 i}(\underline{\beta})}{2}\right)+\sum_{i=1}^{n} \nu_{i 2} \ln \left(1-\frac{\rho A_{2 i}(\underline{\beta})}{2}\right)\right. \\
& \left.-\alpha_{1} e^{-\xi_{11} x_{1 n}} \ln \left(1+\frac{t_{n}}{\alpha_{2}}\right)+\rho\left[\frac{\alpha_{1} \beta}{2} t_{n}^{2} e^{-\left(\xi_{11}+\xi_{21}\right) x_{1 n}} \ln \left(1+\frac{t_{n}}{\alpha_{2}}\right)\right]^{1 / 2}\right\}
\end{aligned}
$$

(b) $\pi\left(\alpha_{2} \mid \underline{\theta}_{\left(\alpha_{2}\right)}, \underline{\nu}, \mathfrak{D}_{\mathrm{t}}\right) \propto \alpha_{2}^{a_{2}-1} e^{-b_{2} \alpha_{1}} \psi_{2}(\underline{\beta})$

onde:

$$
\begin{aligned}
& \psi_{2}(\underline{\beta})=\exp \left\{-\sum_{i=1}^{n} \nu_{i 1} \ln \left(\alpha_{2}+t_{i}\right)+\sum_{i=1}^{n} \nu_{i 1} \ln \left(1-\frac{\rho A_{1 i}(\underline{\beta})}{2}\right)+\sum_{i=1}^{n} \nu_{i 2} \ln \left(1-\frac{\rho A_{2 i}(\underline{\beta})}{2}\right)\right. \\
&\left.-\alpha_{1} e^{-\xi_{11} x_{1 n}} \ln \left(1+\frac{t_{n}}{\alpha_{2}}\right)+\rho\left[\frac{\alpha_{1} \beta}{2} t_{n}^{2} e^{-\left(\xi_{11}+\xi_{21}\right) x_{1 n}} \ln \left(1+\frac{t_{n}}{\alpha_{2}}\right)\right]^{1 / 2}\right\} \\
& \text { (c) } \pi\left(\beta \mid \underline{\theta}_{(\beta)}, \underline{\nu}, \mathfrak{D}_{\mathfrak{t}}\right) \propto \beta^{a_{3}-1} e^{-b_{3 / 3} \beta} \psi_{3}(\underline{\beta})
\end{aligned}
$$

onde:

$$
\begin{aligned}
\iota_{3}(\underline{\beta}) & =\exp \left\{\left(\sum_{i=1}^{n} \nu_{i 2}\right) \ln (3)+\sum_{i=1}^{n} \nu_{i 1} \ln \left(1-\frac{\rho A_{1 i}(\underline{\beta})}{2}\right)+\sum_{i=1}^{n} \nu_{i 2} \ln \left(1-\frac{\rho A_{2 i}(\underline{\beta})}{2}\right)\right. \\
& \left.-\frac{3}{2} t_{n}^{2} e^{-\xi_{21} \cdot x_{1 n}}+\rho\left[\frac{\alpha_{1} \beta}{2} t_{n}^{2} e^{-\left(\xi_{11}+\xi_{21}\right) \cdot x_{1 n} n} \ln \left(1+\frac{t_{n}}{\alpha_{2}}\right)\right]^{1 / 2}\right\}
\end{aligned}
$$


(d) $\pi\left(\xi_{11} \mid \underline{\theta}_{\left(\xi_{11}\right)}, \underline{\nu}, \mathfrak{D}_{\mathrm{t}}\right) \propto \exp \left\{-\frac{1}{2 d_{1}^{2}}\left(\xi_{11}-c_{1}\right)^{2}\right\} \psi_{4}(\underline{\beta})$ onde:

$$
\begin{aligned}
\psi_{4}(\underline{\beta}) & =\exp \left\{-\left(\sum_{i=1}^{n} \nu_{i 1} x_{1 i}\right) \xi_{11}+\sum_{i=1}^{n} \nu_{i 1} \ln \left(1-\frac{\rho A_{1 i}(\underline{\beta})}{2}\right)+\sum_{i=1}^{n} \nu_{i 2} \ln \left(1-\frac{\gamma_{0} A_{2 i}(\underline{\beta})}{2}\right)\right. \\
& \left.-\alpha_{1} e^{-\xi_{11} x_{1 n}} \ln \left(1+\frac{t_{n}}{\alpha_{2}}\right)+\rho\left(x_{1 n}\right)\left[\frac{\alpha_{1} \beta}{2} t_{n}^{2} e^{-\left(\xi_{11}+\xi_{21}\right) x_{1 n}} \ln \left(1+\frac{t_{n}}{\alpha_{2}}\right)\right]^{1 / 2}\right\}
\end{aligned}
$$

(e) $\pi\left(\xi_{21} \mid \underline{\theta}_{\left(\xi_{21}\right)}, \underline{\nu}, \mathfrak{D}_{\mathrm{t}}\right) \propto \exp \left\{-\frac{1}{2 d_{2}^{2}}\left(\xi_{21}-c_{2}\right)^{2}\right\} \psi_{5}(\underline{\beta})$

onde:

$$
\begin{aligned}
& \begin{aligned}
\psi_{5}(\underline{\beta}) & =\exp \left\{\sum_{i=1}^{n} \nu_{i 1} \ln \left(1-\frac{\rho A_{1 i}(\underline{\beta})}{2}\right)-\left(\sum_{i=1}^{n} \nu_{i 2} x_{1 i}\right) \xi_{21}+\left(\sum_{i=1}^{n} \nu_{i 2}\right) \ln \left(1-\frac{\rho A_{2 i}(\underline{\beta})}{2}\right)\right. \\
& \left.-\frac{\beta t_{n}^{2}}{2} e^{-\xi_{21} x_{1 n}}+\rho(1 n)\left[\frac{\alpha_{1} \beta}{2} t_{n}^{2} e^{-\left(\xi_{11}+\xi_{21}\right) x_{1 n}} \ln \left(1+\frac{t_{n}}{\alpha_{2}}\right)\right]^{1 / 2}\right\}
\end{aligned} \\
& \text { (f) } \pi\left(\rho \mid \underline{\theta}_{(\rho)}, \underline{\nu}, \mathfrak{D}_{\mathfrak{t}}\right) \propto \rho^{e_{1}-1}(1-\rho)^{f_{1}-1} \psi_{6}(\underline{\beta})
\end{aligned}
$$

onde:

$$
\begin{aligned}
\psi_{6}(\underline{\beta}) & =\exp \left\{\sum_{i=1}^{n} \nu_{i 1} \ln \left(1-\frac{\rho A_{1 i}(\underline{\beta})}{2}\right)+\sum_{i=1}^{n} \nu_{i 2} \ln \left(1-\frac{\rho A_{2 i}(\underline{\beta})}{2}\right)\right. \\
& \left.+\rho\left[\frac{\alpha_{1} \beta}{2} t_{n}^{2} e^{-\left(\xi_{1}+\xi_{2}\right) x_{n}} \ln \left(1+\frac{t_{n}}{\alpha_{2}}\right)\right]^{1 / 2}\right\}
\end{aligned}
$$

As distribuições condicionais a posteriori dadas em (2.27) não possuem uma distribuição padrão conhecida, de modo que faremos uso do algoritmo de Metropolis-Hastings (Veja, Chib e Greenberg, 1995) para gerar amostras a posteriori de $\alpha_{1}, \alpha_{2}, \beta, \xi_{11}, \xi_{21}$ e $\rho$.

\subsubsection{Análise Bayesiana para o Modelo M4 (superposição de processos independentes na presença de uma covariável)}

Para o modelo M4, considerando a superposição de dois processos PPNH independentes, assumimos as seguintes distribuições a priori para $\alpha_{1}, \alpha_{2}, \beta, \xi_{11}$ e $\xi_{21}$ :

$$
\begin{aligned}
\alpha_{1} & \sim \Gamma\left(a_{1}, b_{1}\right) ; \operatorname{com} a_{1}, b_{1} \text { conhecidas } \\
\alpha_{2} & \sim \Gamma\left(a_{2}, b_{2}\right) ; \operatorname{com} a_{2}, b_{2} \text { conhecidas } \\
3 & \sim \Gamma\left(a_{3}, b_{3}\right) ; \operatorname{com} a_{3}, b_{3} \text { conhecidas } \\
\xi_{11} & \sim N\left(c_{1}, d_{1}\right) ; \operatorname{com} c_{1}, d_{1} \text { conhecidas } \\
\xi_{21} & \sim N\left(c_{2}, d_{2}\right) ; \operatorname{com} c_{2}, d_{2} \text { conhecidas }
\end{aligned}
$$


Da distribuição a posteriori conjunta (2.21) pode-se mostrar que as densidades a posteriori condicionais para a aplicação do algoritmo de Gibbs são dados por:

(a) $\pi\left(\alpha_{1} \mid \underline{\theta}_{\left(\alpha_{1}\right)}, \underline{\nu}, \mathfrak{D}_{\mathrm{t}}\right) \propto \alpha_{1}^{a_{1}-1} e^{-b_{1} \alpha_{1}} \psi_{1}(\underline{\beta})$

onde:

$$
\psi_{1}(\underline{\beta})=\exp \left\{\left(\sum_{i=1}^{n} \nu_{i 1}\right) \ln \left(\alpha_{1}\right)-\alpha_{1} e^{-\xi_{11} x_{1 n}} \ln \left(1+\frac{t_{n}}{\alpha_{2}}\right)\right\}
$$

(b) $\pi\left(\alpha_{2} \mid \underline{\theta}_{\left(\alpha_{2}\right)}, \underline{\nu}, \mathfrak{D}_{\mathrm{t}}\right) \propto \alpha_{2}^{a_{2}-1} e^{-b_{2} \alpha_{1}} \psi_{2}(\underline{\beta})$

onde:

$$
\psi_{2}(\underline{\beta})=\exp \left\{-\sum_{i=1}^{n} \nu_{i 1} \ln \left(\alpha_{2}+t_{i}\right)-\alpha_{1} e^{-\xi_{11} x_{1 n}} \ln \left(1+\frac{t_{n}}{\alpha_{2}}\right)\right\}
$$

(c) $\pi\left(\beta \mid \underline{\theta}_{(\beta)}, \underline{\nu}, \mathfrak{D}_{\mathrm{t}}\right) \propto \beta^{a_{3}-1} e^{-b_{3} \beta} \psi_{3}(\underline{\beta})$

onde:

$$
\psi_{3}(\underline{\beta})=\exp \left\{\left(\sum_{i=1}^{n} \nu_{i 2}\right) \ln (\beta)-\frac{\beta t_{n}^{2}}{2} e^{-\xi_{21} x_{1 n}}\right\}
$$

(d) $\pi\left(\xi_{11} \mid \underline{\theta}_{\left(\xi_{11}\right)}, \underline{\nu}, \mathfrak{D}_{\mathrm{t}}\right) \propto \exp \left\{-\frac{1}{2 d_{1}^{2}}\left(\xi_{11}-c_{1}\right)^{2}\right\} \psi_{4}(\underline{\beta})$

onde:

$$
\psi_{4}(\underline{\beta})=\exp \left\{-\left(\sum_{i=1}^{n} \nu_{i 1} x_{i}\right) \xi_{11}-\alpha_{1} e^{-\xi_{11} x_{1 n}} \ln \left(1+\frac{t_{n}}{\alpha_{2}}\right)\right\}
$$

(e) $\pi\left(\xi_{21} \mid \underline{\theta}_{\left(\xi_{21}\right)}, \underline{\nu}, \mathfrak{D}_{\mathfrak{t}}\right) \propto \exp \left\{-\frac{1}{2 d_{2}^{2}}\left(\xi_{21}-d_{2}\right)^{2}\right\} \psi_{5}(\underline{\beta})$

onde:

$$
\psi_{5}(\underline{\beta})=\exp \left\{-\left(\sum_{i=1}^{n} \nu_{i 2} x_{1 i}\right) \xi_{21}-\frac{3 t_{n}^{2}}{2} e^{-\xi_{21} x_{1 n}}\right\}
$$

As distribuições condicionais a posteriori dadas em (2.29) não possuem uma clistribuição padrão conhecida, de modo que faremos uso do algoritmo de Metropolis-Hastings (Veja, Chib e Greenberg, 1995) para gerar amostras a posteriori de $\alpha_{1}, \alpha_{2}, \beta, \xi_{11}$ e $\xi_{21}$.

De forma similar os cálculos das distribuiçōes condicionais a posteriori foram feitos para a análise dos modelos na presença de duas covariáveis, assumindo distribuições a priori convenientes para cada caso (modelos M5, M6 e M7). 


\subsection{Exemplo 1 (dados simulados com uma covariável)}

Para ilustrar a metodologia apresentada nas seções anteriores consideramos os dados simulados dados na Tabela 2.1 a partir de um PPNH com função intensidade

$$
\lambda(t)=\frac{\alpha_{1} e^{-\xi_{1} x}}{t+\alpha_{2}}+\beta t e^{-\xi_{2} x}
$$

para os valores fixados $\alpha_{1}=20, \alpha_{2}=100, \beta=0.0005, \xi_{1}=0.01$ e $\xi_{2}=65 ; 50$ observações foram geradas considerando uma covariável com valores 2 e 3 . Observar que na geração desses dados assumimos $\rho=0,22$.

Os resultados da análise dos modelos M1, M2, M3 e M4 foram obtidos atráves de métodos MCMC implementado no software WINBUGS (Spiegelhalter et al., 1999) após descartar as 1000 primeiras observações (burn-in); descartamos as 4000 primeiras observações (burn-in) dos modelos M5, M6 e M7. As séries de valores simulados foram avaliados usando os métodos de convergência de Geweke (1992), Raftery \& Lewis (1992) e Gelman \& Rubin (1992), respectivamente, implementados no software CODA (Best et al., 1996). Os resultados para as cadeias geradas foram satisfatórias, e isso pode ser visto graficamente na Figuras 2.2 a 2.8 onde apresentamos o traço e a densidade dos parâmetros de interesse.

Os sumários a posteriori para os parâmetros de interesse estão apresentados na Tabela 2.2 a 2.8 e uma pequena análise de sensitividade foi feita para escolher outros valores de hiperparâmetros, mas estas escolhas não modificaram substancialmente os resultados.

Tabela 2.1: Dados gerados a partir de um processo PPNH

\begin{tabular}{c|c|c|c|c|c|c|c|c|c}
\hline \hline$t_{i}$ & $x_{1 i}$ & $t_{i}$ & $x_{1 i}$ & $t_{1 i}$ & $x_{1 i}$ & $t_{1 i}$ & $x_{1 i}$ & $t_{i}$ & $x_{1 i}$ \\
\hline 4,0205 & 2 & 44,9477 & 3 & 107,9261 & 3 & 242,8235 & 2 & 506,8166 & 3 \\
6,5164 & 3 & 46,9711 & 2 & 115,1079 & 3 & 262,3183 & 3 & 569,1532 & 2 \\
7,8216 & 3 & 58,4492 & 2 & 127,2589 & 3 & 268,7236 & 3 & 608,3752 & 3 \\
13,4633 & 2 & 59,8977 & 3 & 137,9432 & 3 & 277,9392 & 2 & 631,3119 & 2 \\
14,1814 & 2 & 59,9392 & 2 & 140,1109 & 2 & 294,4841 & 3 & 638,8890 & 2 \\
20,3605 & 3 & 63,8705 & 2 & 141,2993 & 2 & 386,1094 & 3 & 696,6598 & 2 \\
34,6135 & 2 & 74,2425 & 3 & 144,5396 & 3 & 386,7154 & 3 & 703,7359 & 3 \\
35,9622 & 2 & 75,0003 & 2 & 155,9962 & 2 & 449,3423 & 2 & 728,8963 & 3 \\
39,0255 & 2 & 79,2846 & 3 & 157,4444 & 3 & 453,4558 & 2 & 745,3901 & 2 \\
42,3482 & 2 & 94,2169 & 3 & 195,7686 & 2 & 505.2807 & 2 & 779,5163 & 2 \\
\hline \hline
\end{tabular}




\subsubsection{Modelo M1 (superposição de processos independentes sem a presença de covariáveis)}

Considerando as distribuições a priori dadas para o modelo M1 (2.22), fixamos diferentes valores para os hiperparâmetros (Vrs Hip), onde os resultados a posteriori estão sumarizados na Tabela 2.2, onde estão apresentadas características tais como média a posteriori, desvio-padrão (DP) e intervalos de credibilidade de $95 \%$ para os parâmetros de interesse. Também apresentamos o fator de escala de redução $\hat{R}$ (Gelman e Rubin, 1992) para os parâmetros $\alpha_{1}, \alpha_{2}$ e $\beta$ do modelo M1. Com valores diferentes dos hiperparâmetros as médias a posteriori são próximas para cada parâmetros do modelo, variando apenas os erros-padrão e os resultados para as cadeias geradas foram satisfatórios, e isso pode ser visto graficamente na Figura 2.2 que apresenta o traço e a densidade dos parâmetros de interesse.

Também observamos convergência do algoritmo a partir dos fatores de escala de redução $\hat{R}$ introduzidos por Gelman e Rubin (1992). As estimativas Bayesianas para os parâmetros $\alpha_{1}, \alpha_{2}$ e $\beta$ estão próximos dos valores usados para simulação dos dados.

Tabela 2.2: Resumos a posteriori para o modelo M1.

\begin{tabular}{c|c|c|c|c|c}
\hline Parâmetro & Vrs. Hip & Média & D.P & Interv. de Credibilidade & $\hat{R}$ \\
\hline \hline \multirow{3}{*}{$\alpha_{1}$} & $\Gamma(600 ; 30)$ & 19,45 & 0,7789 & $(17,94 ; 21,00)$ & 1,006 \\
\cline { 2 - 6 } & $\Gamma(800,40)$ & 19,56 & 0,6836 & $(18,25 ; 20,93)$ & 1,008 \\
\cline { 2 - 6 } & $\Gamma(700 ; 35)$ & 19,49 & 0,7253 & $(18,10 ; 20,95)$ & 1,002 \\
\hline \multirow{3}{*}{$\alpha_{2}$} & $\Gamma(8500 ; 85)$ & 100,00 & 1,073 & $(97,92 ; 102,1)$ & 1,001 \\
\cline { 2 - 6 } & $\Gamma(10000,100)$ & 100,00 & 0,9939 & $(98,09 ; 102,0)$ & 1,002 \\
\cline { 2 - 6 } & $\Gamma(7500 ; 75)$ & 100,00 & 1,155 & $(97,78 ; 102,3)$ & 1,004 \\
\hline \multirow{3}{*}{3} & $\Gamma(130 ; 25500)$ & 0,000468 & 0,00003863 & $(0,0003956 ; 0,0005471)$ & 1,006 \\
\cline { 2 - 6 } & $\Gamma(150,30000)$ & 0,0005239 & 0,0000403 & $(0,0004479 ; 0,0006058)$ & 1,005 \\
\cline { 2 - 6 } & $\Gamma(250 ; 200000)$ & 0,0005462 & 0,0000333 & $(0,000482 ; 0,000613)$ & 1,003 \\
\hline \hline
\end{tabular}

\subsubsection{Modelo M2 (superposição de processos dependentes na presença de uma covariável)}

Considerando as distribuições a priori do modelo M2 (2.24), na Tabela 2.3, apresentamos resumos a posteriori correspondentes aos parâmetros $\alpha_{1}, \alpha_{2}, 3, \xi_{11}, \xi_{21}, \gamma_{0}$ e $\gamma_{1}$, onde fixamos diferentes valores para os hiperparàmetros. Nesta Tabela estão apresentadas características tais como média a posteriori, desvio-padrão (DP) e intervalos de credibilidade de $95 \%$ para os parâmetros de interesse. Também é mostrado o fator de escala de 

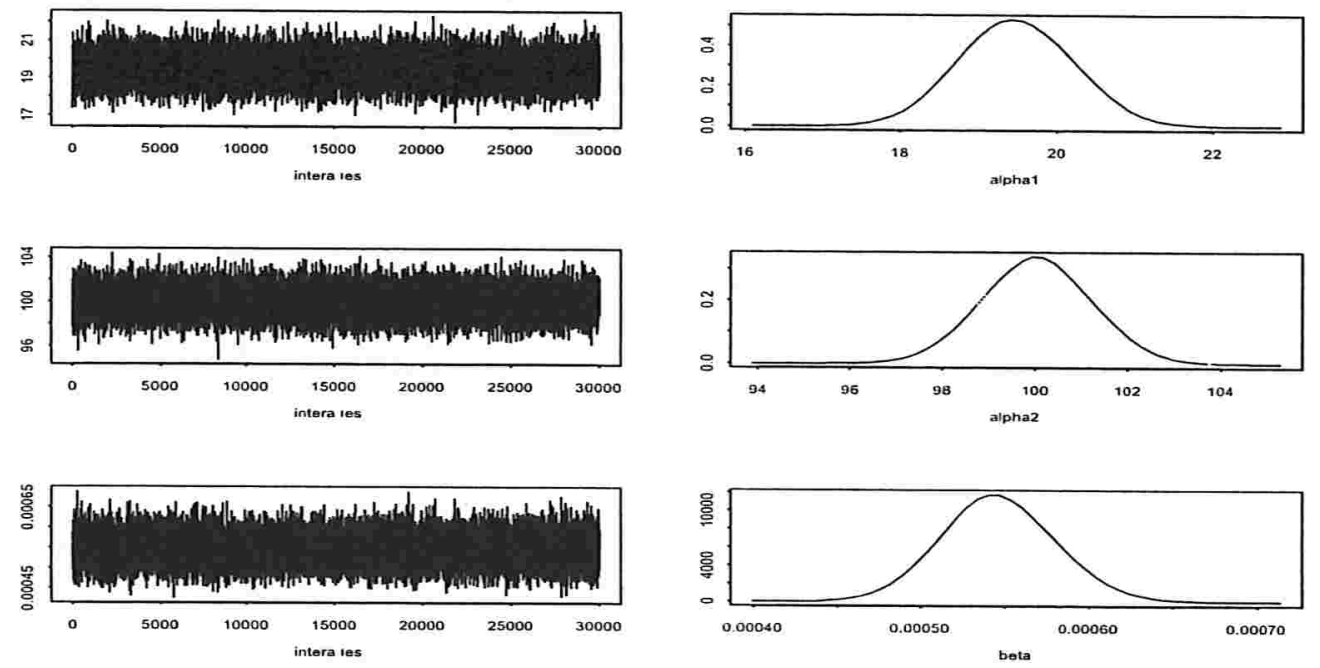

Figura 2.2: $\mathrm{O}$ traço e a Densidade a posteriori de $\alpha_{1}, \alpha_{2}$ e $\beta$

redução $\hat{R}$ para os parâmetros $\alpha_{1}, \alpha_{2}, \beta, \xi_{11}, \xi_{21}, \gamma_{0}, \gamma_{1}$ do modelo M2. Com valores diferentes dos hiperparâmetros as médias a posteriori são próximas para cada parâmetros do modelo, variando apenas os erros-padrão e os resultados para as cadeias geradas foram satisfatórios, e isso pode ser visto graficamente na Figura 2.3 que apresenta o traço e a densidade dos parâmetros de interesse. Também temos $\hat{R}$ menores do que 1,1 para todos os casos, o que indica convergência do algoritmo.

Apesar da convergência do algoritmo, observamos que o parâmetro de regressão $\xi_{11}$ leva à estimativas bem diferentes do valor fixado para simulação dos dados (a escolha dos hiperparâmetros da priori pode levar a diferentes estimadores Bayesianos para $\xi_{11}$ e $\xi_{21}$ ). As estimativas do parâmetros $\alpha_{1}, \alpha_{2}$ e $\beta$ apresentam resultados bem próximos dos valores fixados para simulação dos dados mesmo com valores bem diferentes dos hiperparâmetros das distribuição a priori. Esse problema deve ser consequência da falta de identificabilidade do modelo para os paràmetros de regressão e a presença dos parâmetros de regressão io e $\gamma_{1}$ para a correlação dos PPNHs. Talvez uma reparametrização pode melhorar os resultados obtidos. É importante salientar que uma discriminação Bayesiana deve ser feita para a escolha do melhor modelo. Outra possibilidade é usar outras distribuições a priori para os parâmetros. 
Tabela 2.3: Resumos a posteriori para o modelo M2.

\begin{tabular}{c|c|c|c|c|c}
\hline Parâmetro & Vrs. Hip & Média & D.P & Interv. de Credibilidade & $\hat{R}$ \\
\hline \hline \multirow{6}{*}{$\alpha_{1}$} & $\Gamma(600 ; 30)$ & 20,15 & 0,8173 & $(18,58 ; 21,78)$ & 1,001 \\
\cline { 2 - 6 } & $\Gamma(680,34)$ & 20,11 & 0,7653 & $(18,63 ; 21,64)$ & 1,023 \\
\cline { 2 - 6 } & $\Gamma(800 ; 40)$ & 20,31 & 0,714 & $(18,93 ; 21,73)$ & 1,003 \\
\hline \multirow{5}{*}{$\alpha_{2}$} & $\Gamma(7500 ; 75)$ & 99,95 & 1,155 & $(97,7 ; 102,2)$ & 1,009 \\
\cline { 2 - 6 } & $\Gamma(8500 ; 85)$ & 99,96 & 1,077 & $(97,87 ; 102,1)$ & 1,005 \\
\cline { 2 - 6 } & $\Gamma(10000,100)$ & 99,93 & 0,9926 & $(97,99 ; 101,90)$ & 1,004 \\
\hline \multirow{5}{*}{3} & $\Gamma(112 ; 225000)$ & 0,0004979 & 0,00004729 & $(0,00041 ; 0,0005944)$ & 1,007 \\
\cline { 2 - 6 } & $\Gamma(128 ; 255000)$ & 0,0005689 & 0,0000502 & $(0,000475 ; 0,000672)$ & 1,021 \\
\cline { 2 - 6 }$\xi_{1}$ & $\Gamma(150,30000)$ & 0,0005002 & 0,0004092 & $(0,000423 ; 0,0005837)$ & 1,013 \\
\cline { 2 - 6 } & $N(2 ; 0,066)$ & $-0,0235$ & 0,0724 & $(-0,1624 ; 0,1222)$ & 1,009 \\
\cline { 2 - 6 } & $N(2 ; 0,04)$ & 0,08552 & 0,07775 & $(-0,06174 ; 0,2444)$ & 1,044 \\
\cline { 2 - 6 }$\xi_{2}$ & $N(2 ; 0,0625)$ & $-0,01189$ & 0,07775 & $(-0,153 ; 0,1373)$ & 1,012 \\
\cline { 2 - 6 } & $N(65 ; 10)$ & 64,98 & 3,194 & $(58,74 ; 71,23)$ & 1,039 \\
\cline { 2 - 6 } & $N(65 ; 100)$ & 65,08 & 9,985 & $(45,63 ; 84,72)$ & 1,026 \\
\cline { 2 - 6 } & $N(65 ; 1)$ & 65.0 & 1,012 & $(63,0 ; 66,98)$ & 1,014 \\
\hline \multirow{2}{*}{$\gamma_{0}$} & $B(20 ; 100)$ & 0,1665 & 0.03386 & $(0,1058 ; 0,2384)$ & 1,006 \\
\cline { 2 - 6 } & $B(17 ; 83)$ & 0,1702 & 0,03749 & $(0,1034 ; 0,2503)$ & 1,001 \\
\cline { 2 - 6 } & $B(3 ; 15)$ & 0,167 & 0,08613 & $(0,03783 ; 0,3662)$ & 1,001 \\
\hline \multirow{2}{*}{$\gamma_{1}$} & $B(5 ; 230)$ & 0,02133 & 0,009441 & $(0,006926 ; 0,04326)$ & 1,021 \\
\cline { 2 - 6 } & $B(4,5 ; 225)$ & 0,0196 & 0,009117 & $(0,005981 ; 0,04104)$ & 1,003 \\
\cline { 2 - 6 } & $B(6 ; 300)$ & 0,01956 & 0,007892 & $(0,007285 ; 0,03771)$ & 1,002 \\
\hline \hline
\end{tabular}

\subsubsection{Modelo M3 (superposição de processos dependentes na presença de uma covariável)}

Considerando as distribuições a priori do modelo M3 (2.26), na Tabela 2.4, apresentamos resumos a posteriori correspondentes aos parâmetros $\alpha_{1}, \alpha_{2}, \beta, \xi_{11}, \xi_{21} \mathrm{e}, \rho$, onde fixamos diferentes valores para os hiperparâmetros. Nesta Tabela estão apresentadas características tais como média a posteriori, desvio-padrão (DP) e intervalos de credibilidade de $95 \%$ para os parâmetros de interesse e o fator de redução de escala $\hat{R}$ para cada um dos parâmetros. Com valores diferentes dos hiperparâmetros as médias a posteriori são próximas para cada parâmetros do modelo, variando apenas os erros-padrão e os resultados para as cadeias geradas foram satisfatórios, e isso pode ser visto graficamente na Figura 2.4 que apresenta o traço e a densidade dos parâmetros de interesse. Os valores de $\hat{R}$ também são satisfatórios.

Novamente observamos estimativas Bayesianas para $\alpha_{1}, \alpha_{2}$ e 3 próximos dos valores de geração dos dados. Os paràmetros $\xi_{11}$ e $\xi_{21}$ tem estimativas Bayesianas sensíveis à escollıa 

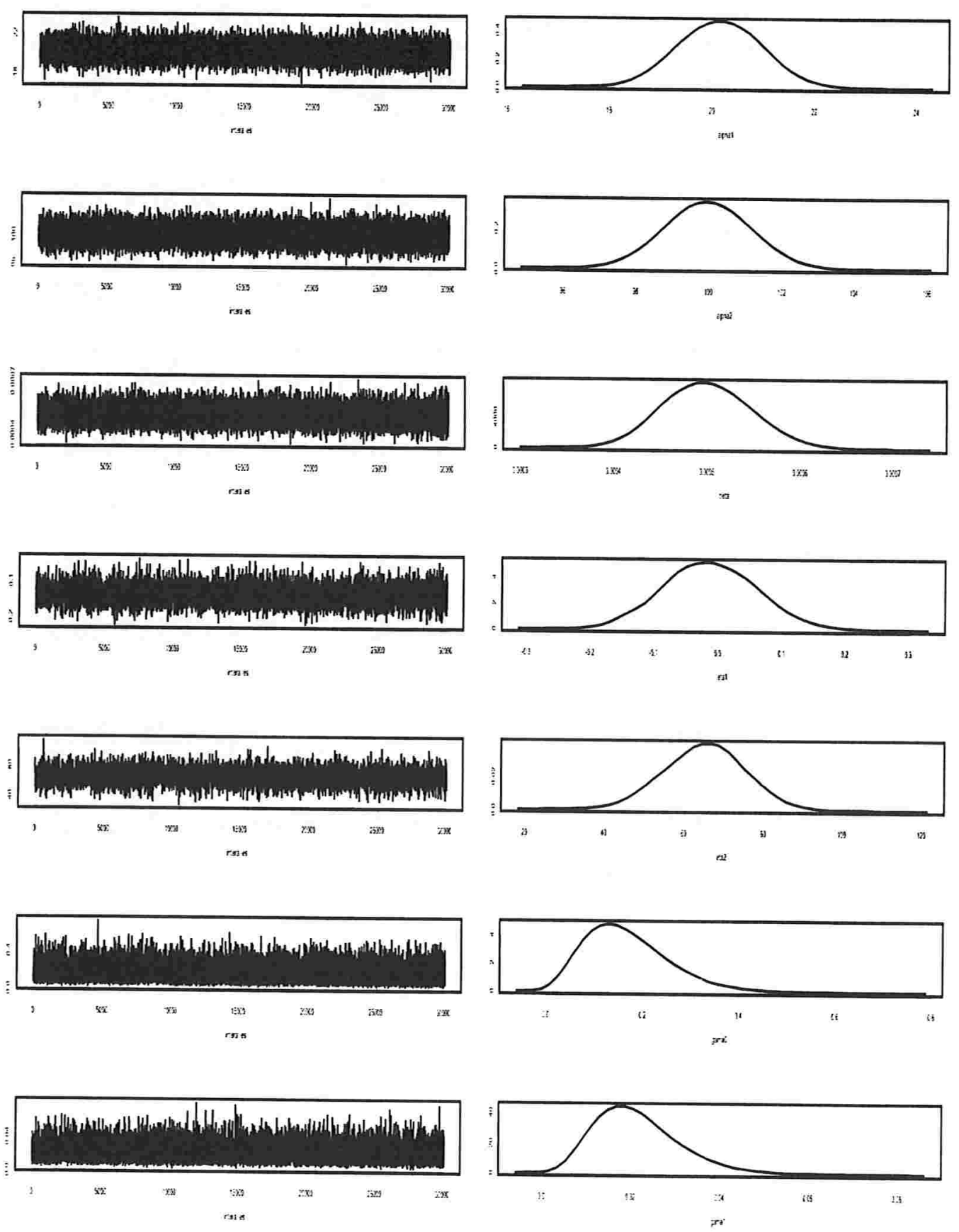

Figura 2.3: O traço e a Densidades a posteriori de $\alpha_{1}, \alpha_{2}, \beta, \xi_{11}, \xi_{21}, \gamma_{0}$ e $\gamma_{1}$

dos valores dos hiperparàmetros da priori. Observamos que o parâmetro de regressão $\xi_{11}$ leva à estimativa bem diferentes do valor fixado para a simulação dos dados. Com as distribuições a priori $N(2 ; 0,058)$ e $N(65 ; 1)$ para $\xi_{11}$ e $\xi_{21}$ respectivamente, as médias a posteriori foram dadas por $\hat{\xi_{11}}=0,01303$ e $\hat{\xi_{21}}=65,02$. Com variância muito grande, para 
Tabela 2.4: Resumos a posteriori para o modelo M3.

\begin{tabular}{c|c|c|c|c|c}
\hline Parâmetro & Vrs. Hip & Média & D.P & Interv. de Credibilidade & $\hat{R}$ \\
\hline \hline \multirow{6}{*}{$\alpha_{1}$} & $\Gamma(400 ; 20)$ & 20,32 & 1,004 & $(18,4 ; 22,34)$ & 1,003 \\
\cline { 2 - 6 } & $\Gamma(800,40)$ & 20,14 & 0,7076 & $(18,77 ; 21,54)$ & 1,006 \\
\cline { 2 - 6 } & $\Gamma(600 ; 30)$ & 20,16 & 0,8168 & $(18,59 ; 21,79)$ & 1,001 \\
\hline \multirow{3}{*}{$\alpha_{2}$} & $\Gamma(5000 ; 50)$ & 99,91 & 1,412 & $(97,18 ; 102,7)$ & 1,002 \\
\cline { 2 - 6 } & $\Gamma(10000 ; 100)$ & 99,96 & 0,9987 & $(98,01 ; 101,9)$ & 1,003 \\
\cline { 2 - 6 } & $\Gamma(7500,75)$ & 99,95 & 1,146 & $(97,74 ; 102,2)$ & 1,003 \\
\hline \multirow{5}{*}{$\xi$} & $\Gamma(150 ; 300000)$ & 0,0005001 & 0,0000409 & $(0,0004231 ; 0,0005835)$ & 1,010 \\
\cline { 2 - 6 } & $\Gamma(42 ; 85000)$ & 0,0004925 & 0,00007608 & $(0,003544 ; 0,004893)$ & 1,006 \\
\cline { 2 - 6 }$\xi_{1}$ & $\Gamma(112,225000)$ & 0,0004977 & 0,00004683 & $(0,00041 ; 0,000593)$ & 1,002 \\
\cline { 2 - 6 } & $N(2 ; 0,055)$ & 0,01303 & 0,07609 & $(-0,1316 ; 0,165)$ & 1,008 \\
\cline { 2 - 6 } & $N(2 ; 0,058)$ & $-0,00331$ & 0,07357 & $(-0,1432 ; 0,1444)$ & 1,002 \\
\cline { 2 - 6 }$\xi_{2}$ & $N(2 ; 1000)$ & $-0,1771$ & 0,0672 & $(-0,3055 ; 0,-0,1797)$ & 1,009 \\
\cline { 2 - 6 } & $N(65 ; 100)$ & 64,88 & 10,01 & $(45,35 ; 84,33)$ & 1,015 \\
\cline { 2 - 6 } & $N(65 ; 10)$ & 65,02 & 9,953 & $(45,52 ; 84,35)$ & 1,017 \\
\cline { 2 - 6 } & $N(65,1)$ & 65,02 & 0,9921 & $(63,07 ; 66,98)$ & 1,039 \\
\hline \multirow{5}{*}{$\rho$} & $B(33,117)$ & 0,22 & 0,03378 & $(0,1575 ; 0,2894)$ & 1,005 \\
\cline { 2 - 6 } & $B(11,39)$ & 0,2199 & 0,05802 & $(0,118 ; 0,3428)$ & 1,005 \\
\cline { 2 - 6 } & $B(22,88)$ & 0,1999 & 0,03776 & $(0,1313 ; 0,2787)$ & 1,004 \\
\hline \hline
\end{tabular}

a priori de $\xi_{21}$, não obtemos convergência do algoritmo Gibbs Sampling. Possivelmente uma reparametrização pode melhorar a convergência do algoritmo.

\subsubsection{Modelo M4 (superposição de processos dependentes na presença de uma covariável)}

Considerando as distribuições a priori do modelo M4 (2.28), na Tabela 2.5 apresentamos resumos a posteriori correspondentes aos parâmetros $\alpha_{1}, \alpha_{2}, \beta, \xi_{11}$ e $\xi_{21}$ onde fixamos diferentes valores para os hiperparâmetros. Nesta Tabela estão apresentadas características tais como média a posteriori, desvio-padrão (DP) e intervalos de credibilidade de $95 \%$ para os parâmetros de interesse e o fator de redução de escala $\hat{R}$ para os parâmetros. Com valores diferentes dos hiperparâmetros as médias a posteriori são próximas para cada parâmetro do modelo, variando apenas os erros-padrão e os resultados para as cadeias geradlas foram satisfatórios, e isso pode ser visto graficamente na Figura 2.5 que apresenta o traço e a densidade dos parâmetros de interesse.

Novamente, verificamos estimativas Bayesianas insensíveis à escolha dos hiperparâmetros das distribuições a priori especialmente para os parâmetros $\alpha_{1}, \alpha_{2}$ e $\beta$. Com os valores clos hiperparâmetros escolhidos para as distribuições a priori para $\xi_{11}$ e $\xi_{21}$, observamos 


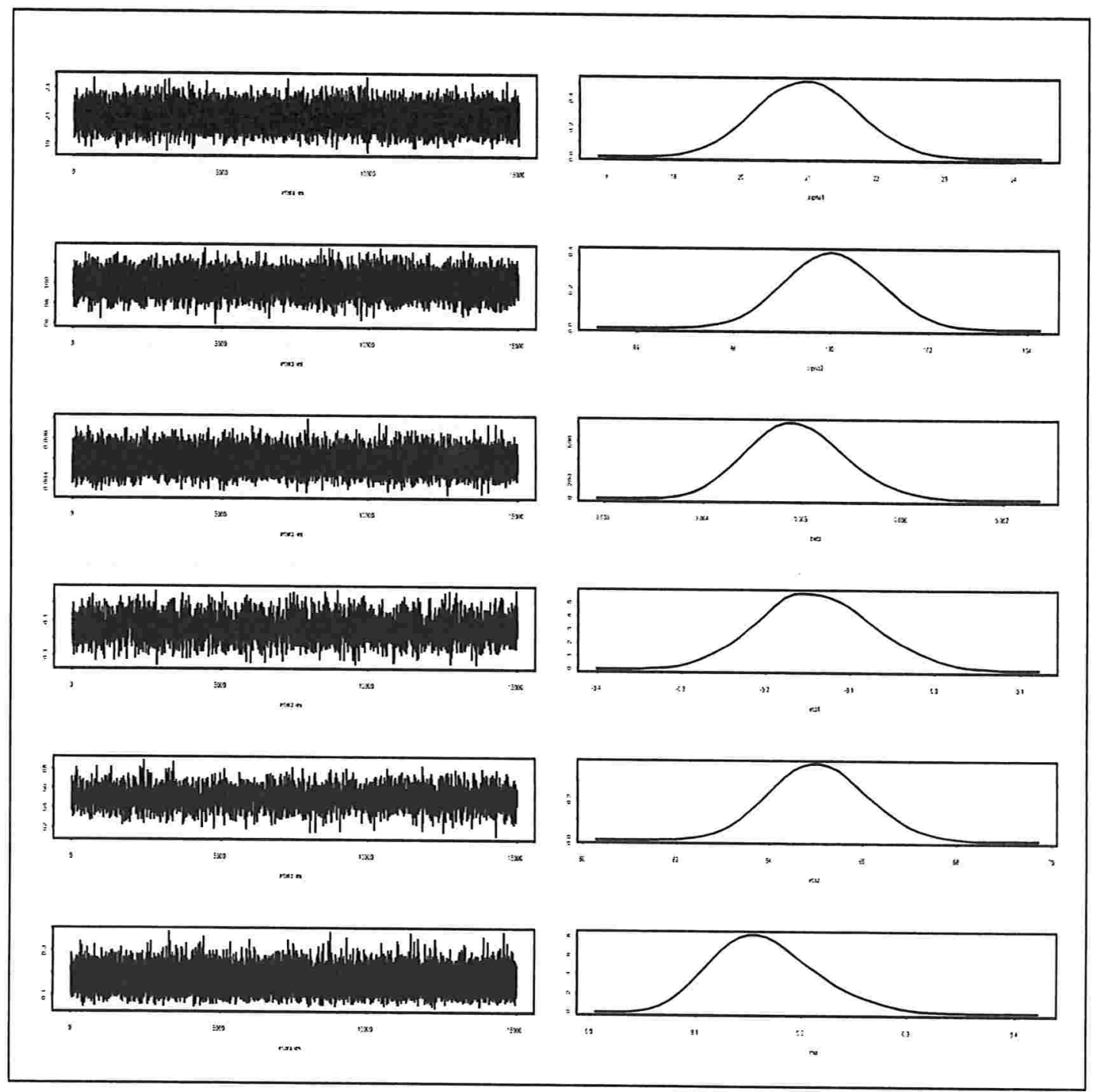

Figura 2.4: O traço e a Densidades a posteriori de $\alpha_{1}, \alpha_{2}, \beta, \xi_{1}, \xi_{2}$ e $\rho$.

convergência do algoritmo Gibbs Sampling, mas um dos parâmetros de regressão $\left(\xi_{11}\right)$ tem estimativa Bayesiana diferente do valor de geração dos dados. Assumindo diferentes valores dos hiperparâmetros da priori para $\xi_{11}$ om a priori $N(2 ; 0,05)$ para $\xi_{11}$, encontramos a estimativa Bayesiana $\dot{\xi_{21}}=0,001117$ (assumindo variância maiores que 1 , tem problema de convergência para algoritmo não foi verificada). Possivelmente é necessário um número bem maior de iterações para se conseguir a convergência do algoritmo; algumas alternativas possíveis: outras distribuições a priori ou uma reparametrização adequada. 
Tabela 2.5: Resumos a posteriori para o modelo M4.

\begin{tabular}{c|c|c|c|c|c}
\hline Parâmetro & Vrs. Hip & Média & D.P & Interv. de Credibilidade & $\hat{R}$ \\
\hline \hline \multirow{3}{*}{$\alpha_{1}$} & $\Gamma(600 ; 30)$ & 20,15 & 0,8185 & $(18,58 ; 21,79)$ & 1,006 \\
\cline { 2 - 6 } & $\Gamma(400,20)$ & 20,32 & 1,002 & $(18,4 ; 22,34)$ & 1,004 \\
\cline { 2 - 6 } & $\Gamma(500 ; 25)$ & 20,26 & 0,8978 & $(18,54 ; 22,05)$ & 1,010 \\
\hline \multirow{2}{*}{$\alpha_{2}$} & $\Gamma(7500 ; 75)$ & 99,96 & 1,146 & $(97,72 ; 102,2)$ & 1,001 \\
\cline { 2 - 6 } & $\Gamma(5000 ; 50)$ & 99,92 & 1,414 & $(97,17 ; 102,7)$ & 1,015 \\
\cline { 2 - 6 } & $\Gamma(10500,105)$ & 99,96 & 0,9734 & $(98,07 ; 101,9)$ & 1,012 \\
\hline \multirow{3}{*}{$\beta$} & $\Gamma(112 ; 225000)$ & 0,0004977 & 0,000047 & $(0,00041 ; 0,0005942)$ & 1,005 \\
\cline { 2 - 6 } & $\Gamma(150 ; 300000)$ & 0,0004998 & 0,0000406 & $(0,000424 ; 0,0005828)$ & 1,011 \\
\cline { 2 - 6 } & $\Gamma(105,210000)$ & 0,0005001 & 0,000049 & $(0,0004092 ; 0,0006006)$ & 1,003 \\
\hline \multirow{3}{*}{$\xi_{21}$} & $N(2 ; 0,0625)$ & $-0,0128$ & 0,07346 & $(-0,1542 ; 0,1345)$ & 1,003 \\
\cline { 2 - 6 } & $N(2 ; 0,0556)$ & 0,01117 & 0,07533 & $(-0,1324 ; 0,1628)$ & 1,008 \\
\hline & $N(65 ; 100)$ & 65,0 & 10,01 & $(45,19 ; 84,67)$ & 1,016 \\
\cline { 2 - 6 } & $N(65 ; 10)$ & 64,99 & 3,176 & $(58,77 ; 71,23)$ & 1,010 \\
\cline { 2 - 6 } & $N(65 ; 1)$ & 65,01 & 0,9932 & $(63,06 ; 66,96)$ & 1,006 \\
\hline \hline
\end{tabular}

\subsection{Exemplo 2 (dados simulados com duas covariáveis)}

\subsubsection{Modelo M5 (superposição de processos dependentes na presença de covariáveis)}

Neste caso, vamos considerar a superposição de um processo Musa e Okumoto com um processo Weibull, dependentes com a presença de duas covariáveis afetando a função intensidade e a correlação $\rho$ dado na forma aditiva $\rho\left(\underline{x_{i}}\right)=\gamma_{0}+\gamma_{1} x_{1 i}+\gamma_{2} x_{2 i}, i=1, \ldots, n$.

Na Tabela 2.6 ,apresentamos os dados simulados a partir de um processo PPNH com função intensidade dado por:

$$
\lambda\left(t_{i}\right)=\frac{\alpha_{1}}{t_{i}+\alpha_{2}} \exp \left\{-\xi_{11} x_{1 i}-\xi_{12} x_{2 i}\right\}+\beta t_{i} \exp \left\{-\xi_{21} x_{1 i}-\xi_{22} x_{2 i}\right\}
$$

para os valores $\alpha_{1}=20, \alpha_{2}=100, \beta=0.0005, \xi_{11}=0.01, \xi_{21}=65, \xi_{21}=-0.80 \mathrm{e}$ $\xi_{22}=0.01 ; 50$ observações foram geradas considerando as covariadas $x_{1 i}$ e $x_{2 i}$ com valores 2 e 3. Observar que na geração desses dados assumimos $\rho=0,7$.

Na Tabela 2.7, apresentamos resumos a posteriori correspondentes aos parâmetros $\alpha_{1}, \alpha_{2}, \beta, \theta, \xi_{21}, \xi_{12}, \xi_{22}, \gamma_{0}, \gamma_{1}$ e $\gamma_{2}$ onde fixamos diferentes valores para os hiperparâmetros. Observar que usamos a reparametrização $\theta=e^{\xi_{11}}$ para conseguirmos convergència do algoritmo.

Nesta Tabela estão apresentadas características tais como média a posteriori, clesvio- 


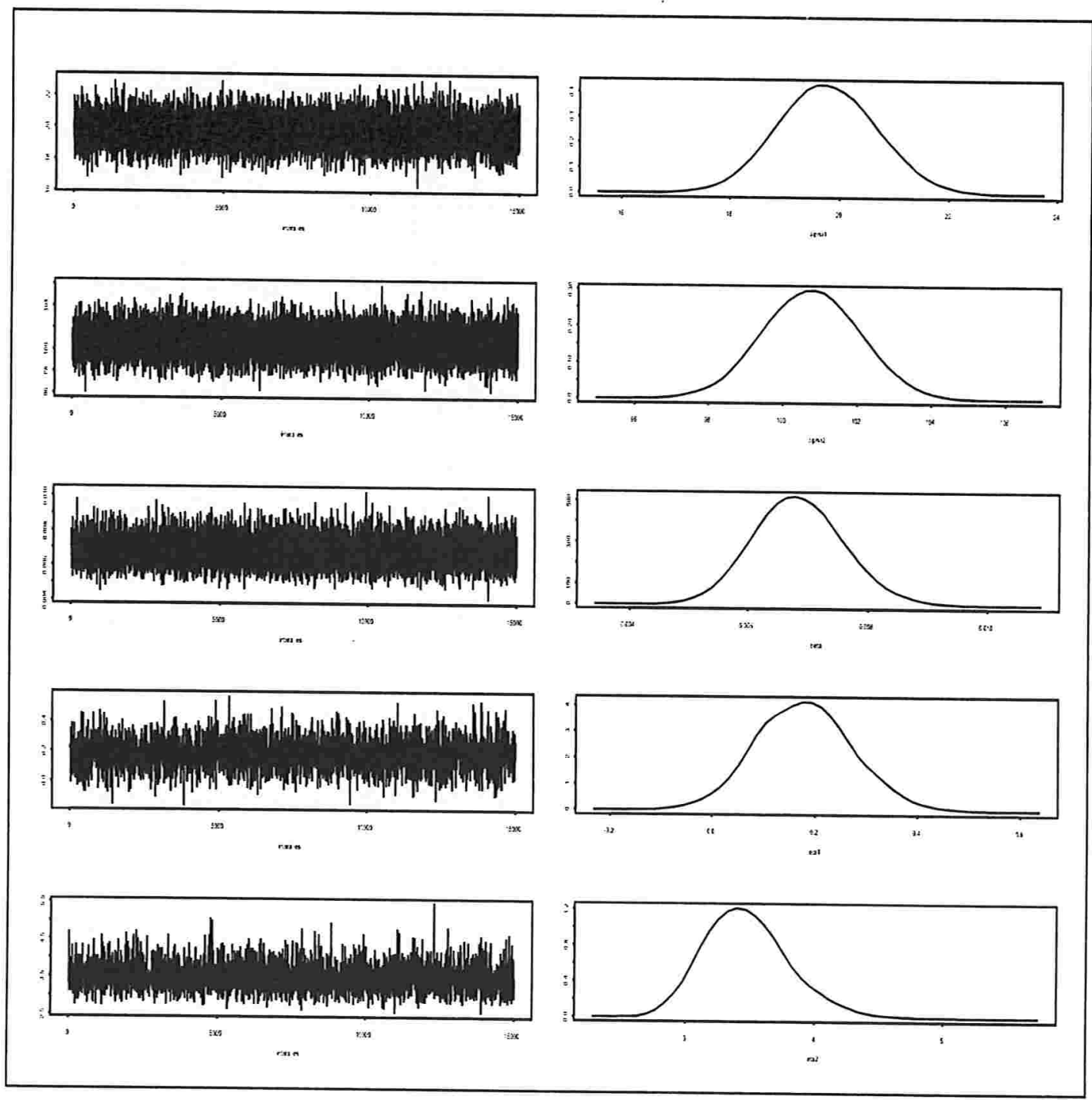

Figura 2.5: O traço e a Densidades a posteriori de $\alpha_{1}, \alpha_{2}, \beta, \xi_{1}, \xi_{2}$ e $\rho$.

padrão (DP) e intervalos de credibilidade de 95 \% para os parâmetros de interesse e o fator de escala de redução $\hat{R}$ para cada parâmetro. Com valores diferentes dos hiperparâmetros as médias a posteriori são próximas para cada parâmetros do modelo, variando apenas os erros-padrão e os resultados para as cadeias geradas foram satisfatórios, e isso pode ser visto graficamente na Figura 2.6 que apresenta o traço e a densidacle dos parâmetros de interesse. 
Tabela 2.6: Dados gerados a partir de um processo PPNH.

\begin{tabular}{c|c|c|c|c|c|c|c|c|c|c|c}
\hline \hline$t_{i}$ & $x_{1 i}$ & $x_{2 i}$ & $t_{i}$ & $x_{1 i}$ & $x_{2 i}$ & $t_{i}$ & $x_{1 i}$ & $x_{2 i}$ & $t_{i}$ & $x_{1 i}$ & $x_{2 i}$ \\
\hline 10,0197 & 3 & 3 & 223,7149 & 2 & 2 & 342,3549 & 3 & 3 & 548,6938 & 2 & 3 \\
10,8673 & 2 & 3 & 237,9540 & 2 & 3 & 346,3754 & 3 & 3 & 551,5108 & 3 & 3 \\
11,4643 & 2 & 3 & 240,0058 & 3 & 3 & 346,9325 & 3 & 3 & 559,7294 & 2 & 2 \\
14,1814 & 2 & 3 & 240,4557 & 3 & 2 & 352,7931 & 3 & 3 & 647,9876 & 2 & 3 \\
39,5923 & 3 & 3 & 253,4639 & 3 & 3 & 379,5120 & 2 & 2 & 658,4159 & 3 & 3 \\
51,1010 & 3 & 2 & 259,5539 & 2 & 2 & 437,2763 & 2 & 2 & 663,2581 & 3 & 3 \\
106,0083 & 2 & 3 & 267,5883 & 3 & 2 & 475,4150 & 3 & 2 & 682,6548 & 3 & 3 \\
108,0043 & 2 & 3 & 294,0067 & 2 & 3 & 476,2711 & 3 & 2 & 689,5649 & 2 & 3 \\
116,8531 & 2 & 3 & 297,5687 & 3 & 3 & 489,7753 & 2 & 3 & 696,4884 & 3 & 3 \\
141,7317 & 3 & 3 & 300,6063 & 3 & 2 & 505,4996 & 2 & 3 & 717,1420 & 3 & 3 \\
200,0150 & 2 & 3 & 300,8858 & 2 & 2 & 522,4391 & 3 & 2 & 730,6319 & 3 & 3 \\
201,7217 & 3 & 2 & 310,4171 & 2 & 3 & 539,2541 & 2 & 3 & & & \\
220,0207 & 2 & 3 & 331,2670 & 3 & 3 & 542,5820 & 3 & 3 & & & \\
\hline \hline
\end{tabular}

\subsubsection{Modelo M6 (superposição de processos dependentes na presença de covariáveis)}

Neste caso, vamos considerar a superposição de um processo Musa e Okumoto com um processo Weibull, dependentes com a presença de duas covariáveis afetando a função intensidade mas não afetando a correlação $\rho$.

Na Tabela 2.8, apresentamos resumos a posteriori correspondentes aos parâmetros $\alpha_{1}, \alpha_{2}, \beta, \xi_{11}, \xi_{21}, \xi_{12}, \xi_{22}$ e $\rho$ onde fixamos os diferentes valores para os hiperparâmetros. Nesta Tabela estão apresentadas características tais como média a posteriori, desviopadrão (DP) e intervalos de credibilidade de $95 \%$ para os parâmetros de interesse o fator estimado da escala de redução $\hat{R}$ para cada parâmetro. Com valores diferentes dos hiperparâmetros as médias a posteriori são próximas para cada parâmetro do modelo, variando apenas os erros-padrão e os resultados para as cadeias foram satisfatórios, e isso pode ser visto graficamente na Figura 2.7 que apresenta o traço e a densidade dos parâmetros de interesse.

\subsubsection{Modelo M7 (superposição de processos independentes na presença de covariáveis)}

Neste caso, vamos considerar a superposição de um processo Musa e Okumoto com um processo Weibull, independentes com a presença de duas covariáveis afetando a função intensidade. 
Tabela 2.7: Resumos a posteriori para o modelo M5.

\begin{tabular}{|c|c|c|c|c|c|}
\hline Parâmetro & Vrs. Hip & Média & D.P & Interv. de Credibilidade & $\hat{R}$ \\
\hline \multirow[t]{3}{*}{$\alpha_{1}$} & $\Gamma(800 ; 40)$ & 20,12 & 0,7048 & $(18,76 ; 21,52)$ & 1,009 \\
\hline & $\Gamma(400,20)$ & 20,28 & 1,008 & $(18,34 ; 22,29)$ & 1,063 \\
\hline & $\Gamma(1600 ; 80)$ & 20,06 & 0,503 & $(19,09 ; 21,06)$ & 1,002 \\
\hline \multirow[t]{3}{*}{$\alpha_{2}$} & $\Gamma(5000 ; 50)$ & 100,1 & 1,417 & $(97,3 ; 102,8)$ & 1,008 \\
\hline & $\Gamma(4000 ; 40)$ & 100,1 & 1,589 & $(96,95 ; 103,2)$ & 1,013 \\
\hline & $\Gamma(10000 ; 100)$ & 99,96 & 0,9992 & $(98,01 ; 101,9)$ & 1,001 \\
\hline \multirow[t]{3}{*}{$\beta$} & $\Gamma(2 ; 4000)$ & 0,0004999 & 0,0003526 & $(0,00006183 ; 0,001385)$ & 1,004 \\
\hline & $\Gamma(150 ; 300000)$ & 0,0005002 & 0,00004075 & $(0,0004232 ; 0,0005828)$ & 1,004 \\
\hline & $\Gamma(50 ; 100000)$ & 0,0005 & 0,00007083 & $(0,000371 ; 0,0006477)$ & 1,001 \\
\hline \multirow[t]{3}{*}{$\theta$} & $N(2 ; 5)$ & $-0,9682$ & 0,2255 & $(-1,432 ;-0,5508)$ & 1,012 \\
\hline & $N(0 ; 100)$ & $-0,2756$ & 0,08906 & $(-0,4512 ;-01005)$ & 1,054 \\
\hline & $N(0 ; 50)$ & $-0,4611$ & 0,118 & $(-0,6939 ;-0,227)$ & 1,025 \\
\hline \multirow[t]{3}{*}{$\xi_{21}$} & $N(65 ; 1)$ & 64,99 & 0,994 & $(63,05 ; 66,94)$ & 1,019 \\
\hline & $N(60 ; 10)$ & 60,0 & 03209 & $(59,37 ; 60,63)$ & 1,024 \\
\hline & $N(70 ; 20)$ & 70,0 & 0,2211 & $(69,57 ; 70,45)$ & 1,051 \\
\hline \multirow[t]{3}{*}{$\xi_{12}$} & $N(0.01 ; 10)$ & $-0,2571$ & 0,07762 & $(-0,4143 ;-0,1107)$ & 1,042 \\
\hline & $N(0,01 ; 1)$ & $-0,5114$ & 0,06756 & $(-0,6438 ;-0,3778)$ & 1,053 \\
\hline & $N(0 ; 0,01)$ & $-1,197$ & 0,07125 & $(-1,341 ;-1,06)$ & 1,037 \\
\hline \multirow[t]{3}{*}{$\xi_{22}$} & $B(10 ; 990)$ & 0,01002 & 0,003172 & $(0,004778 ; 0,01711$ & 1,007 \\
\hline & $B(1 ; 99)$ & 0,009969 & 0,009904 & $(0,0002545 ; 0,03652)$ & 1,004 \\
\hline & $B(2 ; 198)$ & 0,01004 & 0,007008 & $(0,00124 ; 0,02773)$ & 1,008 \\
\hline \multirow[t]{3}{*}{$\gamma_{0}$} & $B(4,1 ; 21,9)$ & 0,1581 & 0,07039 & $(0,04737 ; 0,3173)$ & 1,012 \\
\hline & $B(6 ; 34)$ & 0,1501 & 0,05578 & $(0,05861 ; 0,2731)$ & 1,035 \\
\hline & $B(18 ; 102)$ & 0,1498 & 0,03221 & $(0,09284 ; 0,2186)$ & 1,002 \\
\hline \multirow[t]{3}{*}{$\gamma_{1}$} & $B(8,3 ; 41,7)$ & 0,1658 & 0,05201 & $(0,07715 ; 0,2789)$ & 1,001 \\
\hline & $B(11 ; 59)$ & 0,1572 & 0,04288 & $(0,08288 ; 0,2504)$ & 1,007 \\
\hline & $B(14 ; 76)$ & 0,1556 & 0,03794 & $(0,08918 ; 0,2372)$ & 1,006 \\
\hline \multirow[t]{3}{*}{$\gamma_{2}$} & $B(16,7 ; 83,3)$ & 0,1667 & 0,03723 & $(0,1005 ; 0,2462)$ & 1,006 \\
\hline & $B(20 ; 100)$ & 0,1665 & 0,03379 & $(0,1055 ; 0,2382)$ & 1,035 \\
\hline & $B(5 ; 25)$ & 0,1667 & 0,06697 & $(0,05845 ; 0,318)$ & 1,022 \\
\hline
\end{tabular}

Na Tabela 2.9, apresentamos resumos a posteriori correspondentes aos parâmetros $\alpha_{1}, \alpha_{2}, \beta, \xi_{11}, \xi_{21}, \xi_{12}$ e $\xi_{22}$ onde fixamos os diferentes valores para os hiperparâmetros. Nesta Tabela estão apresentadas características tais como média a posteriori, desviopadrão (DP) e intervalos de credibilidade de $95 \%$ para os parâmetros de interesse e a hipótese estimada da escala de redução $\hat{R}$ para cada parâmetro. Com valores diferentes dos hiperparàmetros as médias a posteriori são próximas para cada parâmetros do modelo, variando apenas os erros-padrão e os resultados para as cadeias foram satisfatórios, e isso pode ser visto graficamente na Figura 2.8 que apresenta o traço e a densidade dos parâmetros de interesse. 

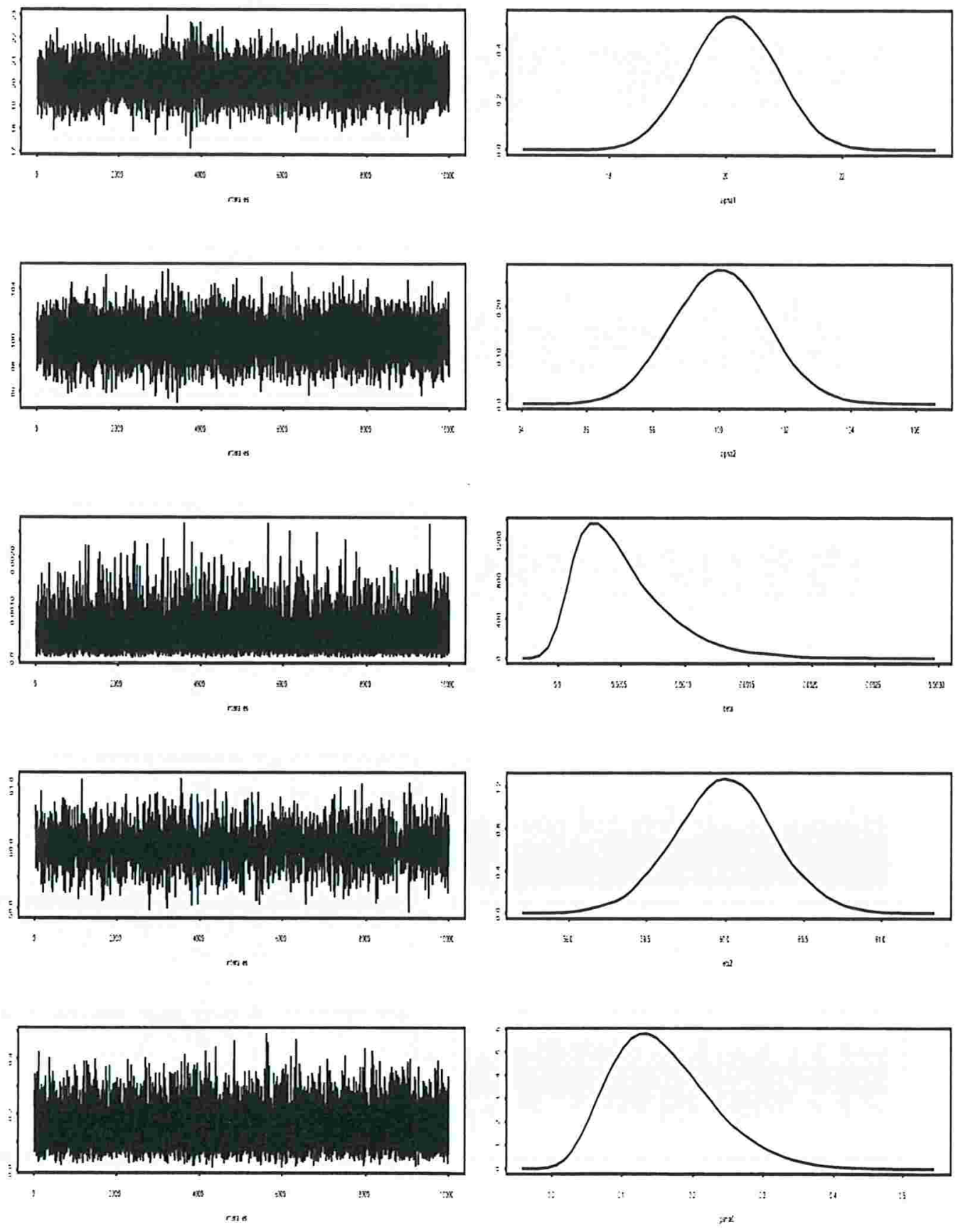

Figura 2.6: O traço e a Densidades a posteriori de $\alpha_{1}, \alpha_{2}, 3, \theta, \xi_{21}, \gamma_{0}, \gamma_{1}, \gamma_{2}, \xi_{12}$ e $\xi_{22}$

\subsection{Seleção de Modelos}

Apresentamos nas Tabelas 2.10 e 2.11 os resultados obtidos dos modelos analisados pelos critérios AIC, BIC, DIC para o produto das preditivas apresentado na seção (1.3) 


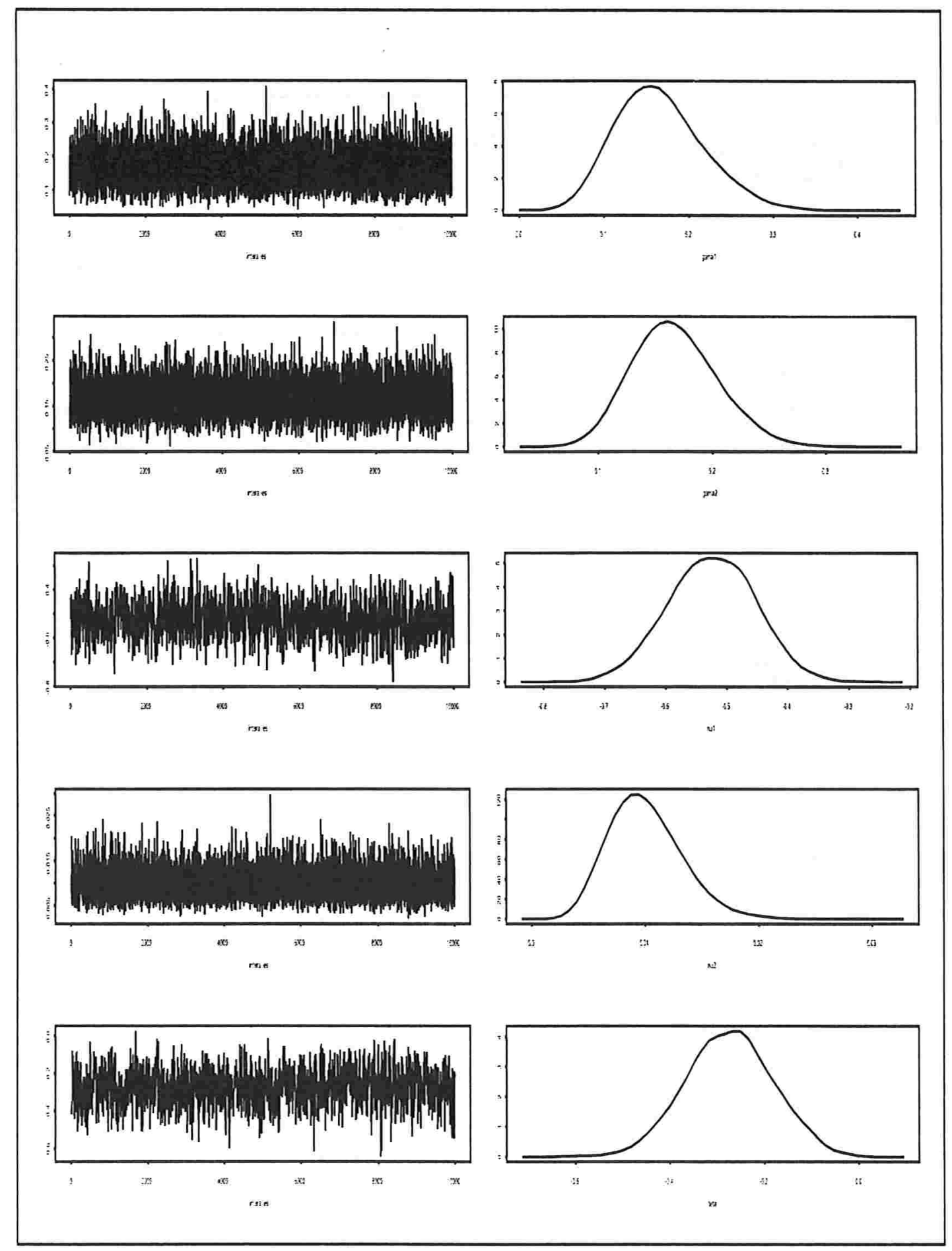

Figura 2.7: O traço e a Densidades a posteriori de $\alpha_{1}, \alpha_{2}, \beta, \theta, \xi_{21}, \gamma_{0}, \gamma_{1}, \gamma_{2}, \nu_{1}$ e $\nu_{2}$.

Da Tabela 2.10, observamos para os modelos M2, M3 e M4 na presença de uma covariável, que os modelos M3 e M4 apresentam ajustes melhores pelo critério DIC da superposição de um processo de Musa e Okumoto com o processo de Weibull. Enquanto o modelo M2 tem um ajuste melhor pelo critérios BIC, observamos menor valor o AIC para 
Tabela 2.8: Resumos a posteriori para o modelo M6.

\begin{tabular}{|c|c|c|c|c|c|}
\hline Parâmetro & Vrs. Hip & Média & D.P & Interv. de Credibilidade & $\hat{R}$ \\
\hline \multirow[t]{3}{*}{$\alpha_{1}$} & $\Gamma(800 ; 40)$ & 20,12 & 0,7045 & $(18,76 ; 21,52)$ & 1,006 \\
\hline & $\Gamma(400,20)$ & 20,27 & 1,004 & $(18,36 ; 22,28)$ & 1,018 \\
\hline & $\Gamma(1600 ; 80)$ & 20,06 & 0,4974 & $(19,10 ; 21,06)$ & 1,016 \\
\hline \multirow[t]{3}{*}{$\alpha_{2}$} & $\Gamma(5000 ; 50)$ & 100,1 & 1,412 & $(97,32 ; 102,8)$ & 1,003 \\
\hline & $\Gamma(4000 ; 40)$ & 100,1 & 1,587 & $(96,96 ; 103,20)$ & 1,005 \\
\hline & $\Gamma(10000 ; 100)$ & 100,0 & 0,9993 & $(98,09 ; 102,0)$ & 1,009 \\
\hline \multirow[t]{3}{*}{$\beta$} & $\Gamma(50 ; 100000)$ & 0,0005 & 0,0000703 & $(0,0003718 ; 0,0006477)$ & 1,008 \\
\hline & $\Gamma(150 ; 30000)$ & 0,000499 & 0,00004065 & $(0,0004232 ; 0,0005821)$ & 1,011 \\
\hline & $\Gamma(2,4000)$ & 0,0005025 & 0,0003556 & $(0,00005945 ; 0,001408)$ & 1,005 \\
\hline \multirow[t]{3}{*}{$\theta$} & $N(0 ; 50)$ & $-0,4575$ & 0,1173 & $(-0,6919 ;-0,2337)$ & 1,007 \\
\hline & $N(1 ; 60)$ & $-0,2765$ & 0,08798 & $(-0,4479 ;-0,1036)$ & 1,017 \\
\hline & $N(0 ; 10)$ & $-1,183$ & 0,2123 & $(-1,605 ;-0,7731)$ & 1,027 \\
\hline \multirow[t]{3}{*}{$\xi_{21}$} & $N(65 ; 1)$ & 65,0 & 1,008 & $(63,02 ; 66,99)$ & 1,018 \\
\hline & $N(60 ; 10)$ & 60 & 0,3175 & $(59,38 ; 6061)$ & 1,021 \\
\hline & $N(60 ; 10)$ & 60,0 & 0,3133 & $(59,39 ; 60,63)$ & 1,005 \\
\hline \multirow[t]{3}{*}{$\xi_{12}$} & $N(0,01 ; 10)$ & $-0,5132$ & 0,06756 & $(-0,6482 ;-0,3784)$ & 1,049 \\
\hline & $N(0 ; 10)$ & $-0,8485$ & 0,09791 & $(-1,051 ;-0,6654)$ & 1,036 \\
\hline & $N(0 ; 0,01)$ & $-1,2$ & 0,07084 & $(-1,338 ;-1,061)$ & 1,088 \\
\hline \multirow[t]{3}{*}{$\xi_{22}$} & $B(10 ; 990)$ & 0,006037 & 0,006009 & $(0,0001552 ; 0,02223)$ & 1,015 \\
\hline & $B(1 ; 99)$ & 0,009997 & 0,009939 & $(0,0002493 ; 0,03672)$ & 1,017 \\
\hline & $B(2 ; 198)$ & 0,009904 & 0,00697 & $(0,001212 ; 0,02735)$ & 1,013 \\
\hline \multirow[t]{3}{*}{$\rho$} & $B(4,1 ; 21,9)$ & 0,1576 & 0,07016 & $(0,04779 ; 0,3172)$ & 1,004 \\
\hline & $B(6 ; 34)$ & 0,1501 & 0,05598 & $(0,05897 ; 0,2744)$ & 1,007 \\
\hline & $B(18 ; 102)$ & 0,15 & 0,003266 & $(0,09133 ; 0,2186)$ & 1,006 \\
\hline
\end{tabular}

os modelos M3 e M4. Em relação às preditivas ordenadas (ver Tabela 2.11) observarmos que o modelo M3 é o melhor modelo ajustado para os dados. Para os modelos M5, M6 e M7 na presença de duas covariáveis,a seleção pelo critério AIC indica que o modelo M6 tem um ajuste melhor, o modelo M7 é melhor pelo BIC e os modelos M5 e M6 apresentam os melhores ajustes pelo DIC. Em relação às preditivas ordenadas (ver Tabela 2.11), o melhor modelo é dado pelo modelo M7. 


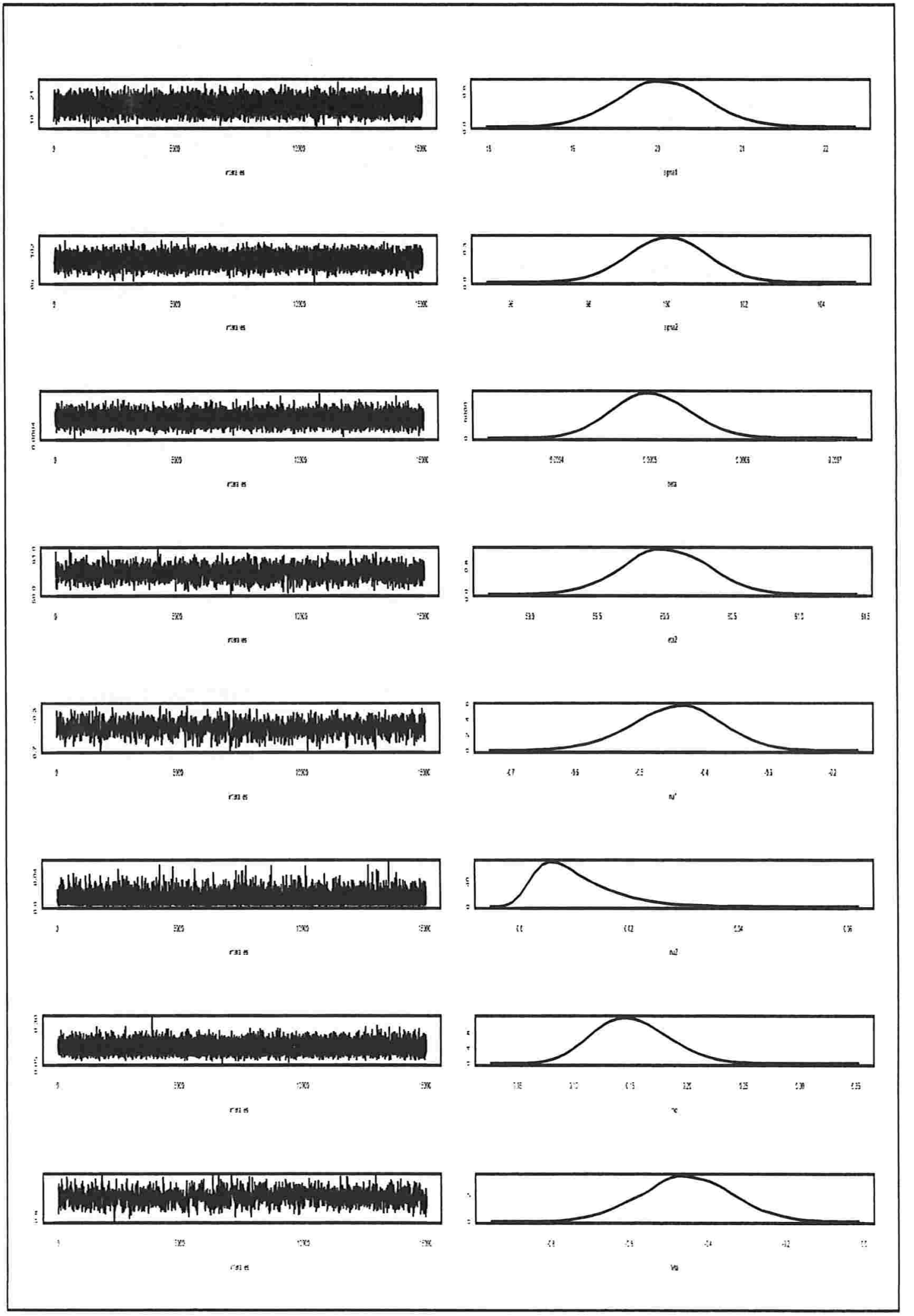

Figura 2.8: O traço e a Densidades a posteriori de $\alpha_{1}, \alpha_{2}, \beta, \theta, \xi_{21}, \xi_{12}, \xi_{22}$ e $\rho$. 
Tabela 2.9: Resumos a posteriori para o modelo M7.

\begin{tabular}{c|c|c|c|c|c}
\hline Parâmetro & Vrs. Hip & Média & D.P & Interv. de Credibilidade & $\hat{R}$ \\
\hline \hline \multirow{5}{*}{$\alpha_{1}$} & $\Gamma(400 ; 20)$ & 20,12 & 1,006 & $(18,19 ; 22,14)$ & 1,001 \\
\cline { 2 - 6 } & $\Gamma(800,40)$ & 20,06 & 0,7117 & $(18,69 ; 21,47)$ & 1,003 \\
\cline { 2 - 6 } & $\Gamma(1600 ; 80)$ & 20,07 & 0,8151 & $(18,48 ; 21,69)$ & 1,016 \\
\hline \multirow{5}{*}{$\alpha_{2}$} & $\Gamma(4000 ; 40)$ & 99,96 & 1,582 & $(96,9 ; 103,10)$ & 1,025 \\
\cline { 2 - 6 } & $\Gamma(5000 ; 50)$ & 99,97 & 1,422 & $(97,2 ; 102,8)$ & 1,008 \\
\cline { 2 - 6 } & $\Gamma(1000 ; 100)$ & 99,98 & 0,9949 & $(98,03 ; 101,9)$ & 1,011 \\
\hline \multirow{5}{*}{$\beta$} & $\Gamma(150 ; 300000)$ & 0,0005833 & 0,00004464 & $(0,0004994 ; 0,0006741)$ & 1,008 \\
\cline { 2 - 6 } & $\Gamma(50 ; 100000)$ & 0,0007503 & 0,00008571 & $(0,0005928 ; 0,000928)$ & 1,016 \\
\cline { 2 - 6 } & $\Gamma(2 ; 4000)$ & 0,0006748 & 0,001297 & $(0,00445 ; 0,009524)$ & 1,001 \\
\hline \multirow{5}{*}{$\xi$} & $N(0 ; 50)$ & $-0,276$ & 0,1238 & $(-0,5229 ;-0,03612)$ & 1,005 \\
\cline { 2 - 6 } & $N(0 ; 100)$ & $-0,1593$ & 0,09266 & $(-0,3427 ;-0,02105)$ & 1,025 \\
\cline { 2 - 6 } & $N(0 ; 80)$ & $-0,1902$ & 0,1033 & $(-0,3953 ;-0,01359)$ & 1,034 \\
\cline { 2 - 6 } & $N(60 ; 10)$ & 53,65 & 0,319 & $(53,03 ; 54,28)$ & 1,021 \\
\cline { 2 - 6 } & $N(70 ; 10)$ & 63,65 & 0,3177 & $(63,02 ; 64,27)$ & 1,026 \\
\cline { 2 - 6 } & $N(70 ; 20)$ & 66,82 & 0,2242 & $(66,38 ; 67,26)$ & 1,014 \\
\hline \multirow{5}{*}{$\xi_{12}$} & $N(0 ; 0,01)$ & $-0,2931$ & 0,09649 & $(-0,4798 ;-0,1043)$ & 1,004 \\
\cline { 2 - 6 } & $N(0 ; 0,1)$ & $-0,2916$ & 0,09534 & $(-0,4763 ;-0,1022)$ & 1,056 \\
\cline { 2 - 6 } & $N(0,01 ; 1)$ & $-0,3521$ & 0,08946 & $(-0,525 ;-0,176)$ & 1,008 \\
\hline \multirow{3}{*}{$\xi_{22}$} & $B(1 ; 99)$ & 0,005995 & 0,006029 & $(0,0001504 ; 0,02232)$ & 1,015 \\
\cline { 2 - 6 } & $B(10 ; 990)$ & 0,009362 & 0,002943 & $(0,004522 ; 0,01598)$ & 1,004 \\
\cline { 2 - 6 } & $B(2 ; 198)$ & 0,007485 & 0,005266 & $(0,0009036 ; 0,02071)$ & 1,019 \\
\hline \hline
\end{tabular}

Tabela 2.10: Resultados obtidos para os Critérios AIC BIC DIC.

\begin{tabular}{c|c|c|c|c|c|c}
\hline & Modelo & N $^{\circ}$ Obs. & AIC & BIC & DIC & N. $^{\circ}$ Parâmetros \\
\hline \hline s/cov & M1 & 50 & 19,01774 & 32,4019 & 1000530,00 & 4 \\
\hline uma & M2 & 50 & 21,12132 & 34.50549 & 1000360,00 & 7 \\
\cline { 2 - 7 } $\begin{array}{c}\text { covariá- } \\
\text { vel }\end{array}$ & M3 & 50 & 16,25305 & 25.81316 & 1000350,00 & 6 \\
\cline { 2 - 7 } $\begin{array}{c}\text { Duas } \\
\text { covariá- } \\
\text { veis }\end{array}$ & M5 & 50 & 16,25305 & 24,59192 & 1000350,00 & 5 \\
\cline { 2 - 7 } & M6 & 50 & 24,33611 & 43,45634 & 1000390,00 & 10 \\
\hline
\end{tabular}

Tabela 2.11: Resultados obtidos para Preditivas.

\begin{tabular}{|c|c|c|c|}
\hline & Modelo & $\mathrm{N}^{\circ}$ Obs. & Preditivas \\
\hline $\mathrm{s} / \mathrm{cov}$ & M1 & 50 & $4,6458 e^{-9}$ \\
\hline \multirow{3}{*}{$\begin{array}{c}\text { Uma } \\
\text { covariá- } \\
\text { vel }\end{array}$} & $\backslash 2$ & 50 & $8,83066 e^{-10}$ \\
\hline & 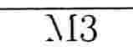 & 50 & $1,4105 e^{-8}$ \\
\hline & . $[14$ & 50 & $4,2308 e^{-18}$ \\
\hline \multirow{3}{*}{$\begin{array}{c}\text { Duas } \\
\text { covariá- } \\
\text { veis }\end{array}$} & 215 & 50 & $3,6378 e^{-17}$ \\
\hline & 216 & 50 & $9,37238 e^{-12}$ \\
\hline & .17 & 50 & $1,1714 e^{-10}$ \\
\hline
\end{tabular}




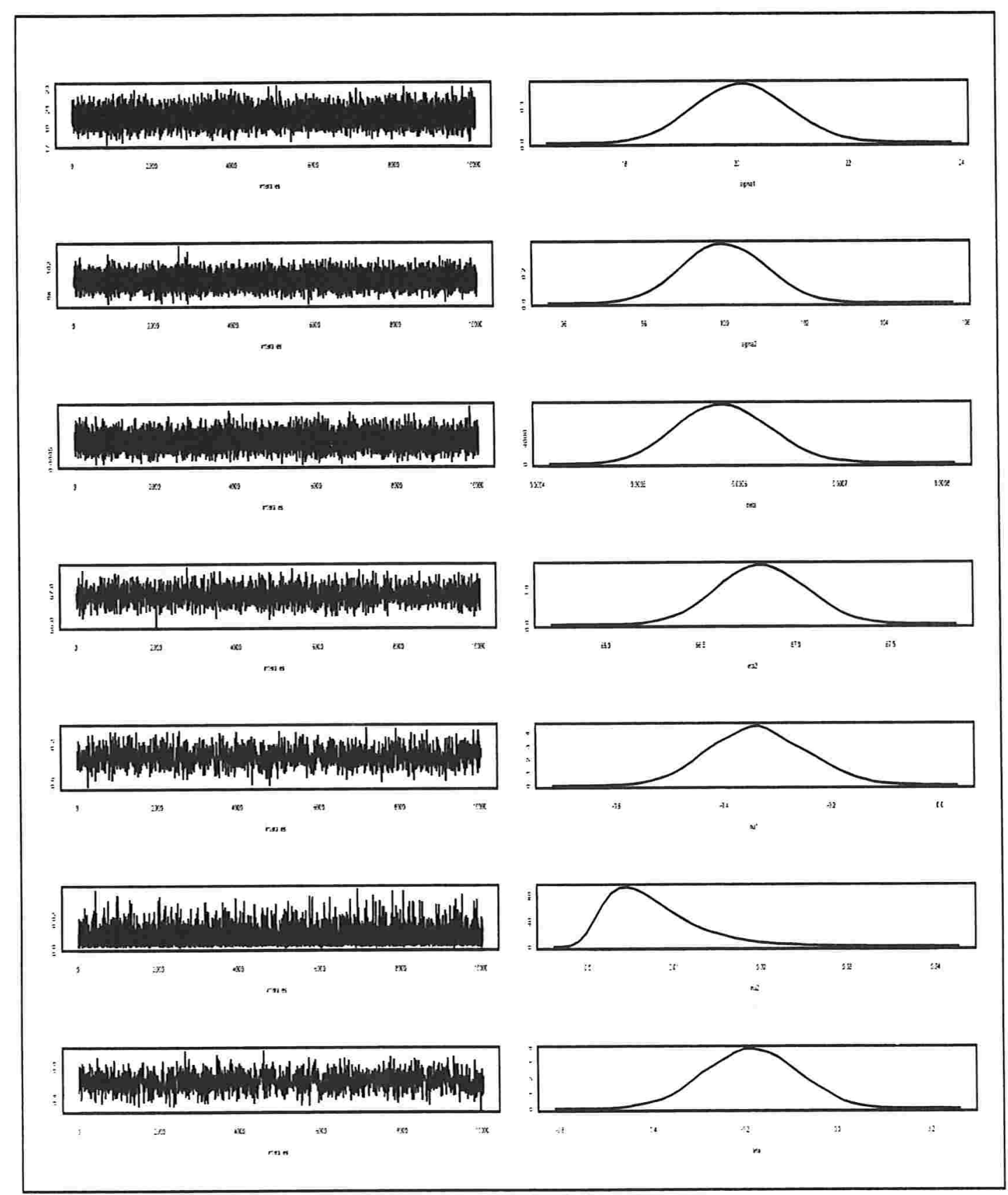

Figura 2.9: O traço e a Densidades a posteriori de $\alpha_{1}, \alpha_{2}, 3, \xi_{11}, \xi_{21}, \xi_{12}$ e $\xi_{22}$. 


\section{Capítulo 3}

\section{Superposição de Processos de Poisson Não-homogêneos Polinomiais Dependentes}

Apresentamos neste capítulo alguns modelos superposição de PPNH polinomiais dependentes sob o ponto de vista Bayesiano. Damos alguns exemplos de aplicações dos conjuntos de dados simulados a partir de PPNH.

Nossa contribuição neste capítulo é a incorporação da dependência de superposição de PPNH na presença de uma ou mais covariáveis. Iniciamente introduzimos um modelo genérico para a superposição de PPNH polinomiais dependentes.

\subsection{Descrição do modelo genérico}

Consideramos um PPNH polinomial com função intensidade dada por

$$
\lambda(t)=\beta_{0}+\beta_{1} t+\cdots+\beta_{J-1} t^{J-1},
$$

$\operatorname{com} J=2,3, \cdots$.

Seja $D_{t}$ o conjunto de dados observados até o tempo $t$ e $n$ épocas observadas:

$0<t_{1}<t_{2}<\ldots<t_{n}<t$,( modelo de tempo truncado).

A seguir vamos descrever o caso onde assumimos $J=2$.

\subsection{A Superposição de dois PPNH Polinomiais de Primeiro Grau}

Neste caso vamos apresentar alguns casos especiais de modelos de PPNHs na presença ou não de uma covariável. 
Modelo M1 (superposição de processos independentes sem a presença de covariáveis)

Este modelo é um caso especial, onde assumimos a superposição de dois PPNHs polinomiais independentes e a função intensidade deste processo é dada por

$$
\lambda\left(t_{i}\right)=\lambda_{1}\left(t_{i}\right)+\lambda_{2}\left(t_{i}\right)=\beta_{0}+\beta_{1} t_{i}
$$

Neste caso temos uma intensidade linear.

A função de valor médio deste processo de superposição é dada por:

$$
m\left(t_{n}\right)=m_{1}\left(t_{n}\right)+m_{2}\left(t_{n}\right)=\beta_{0} t_{n}+\frac{\beta_{1} t_{n}^{2}}{2} .
$$

Assim, de (1.18) a função de verossimilhança com $\rho=0$ é dada por,

$$
L\left(\underline{\beta} \mid \mathfrak{D}_{\mathfrak{t}}\right)=\prod_{i=1}^{n}\left[\beta_{0}+\beta_{1} t_{i}\right] \exp \left\{-\left[\beta_{0} t_{n}+\frac{\beta_{1} t_{n}^{2}}{2}\right]\right\} .
$$

Denotamos o vetor de parâmetros deste modelo por $\underline{\beta}=\left(\beta_{0}, \beta_{1}\right)$, e a seguir vamos considerar PPNHs polinomiais dependentes.

Modelo M2 (superposição de processos dependentes sem a presença de covariáveis)

Neste modelo vamos considerar o caso onde a covariavél $x_{i}$ não afeta a função de intensidade e o parâmetro de correlação $\rho$, isto é, consideramos $\rho$ constante.

Considerando a mesma função intensidade do modelo M1 (3.2) a função de verossimilhança (1.18) com $\rho$ constante é reescrita como,

$$
\begin{aligned}
L(\underline{\beta}, \rho) & =\prod_{i=1}^{n}\left[\lambda_{1}\left(t_{i}\right)+\lambda_{2}\left(t_{i}\right)\right]\left[1-\frac{\rho W\left(t_{i}\right)}{2}\right] \\
& \times \exp \left\{-\left[m_{1}\left(t_{n}\right)+m_{2}\left(t_{n}\right)-\rho\left(m_{1}\left(t_{n}\right) m_{2}\left(t_{n}\right)\right)^{1 / 2}\right]\right\},
\end{aligned}
$$

onde $W\left(t_{i}\right)$ é definido em (1.19), $m_{1}\left(t_{n}\right)$ e $m_{2}\left(t_{n}\right)$ em (3.3) respectivamente. Denotamos o vetor de parâmetros deste modelo por $\underline{\beta}=\left(\beta_{0}, \beta_{1}, \rho\right)$.

Modelo M3 (superposição de processos dependentes na presença de uma covariável)

Assumir agora que as funções intensidades correspondentes assumem um efeito multiplicativo da covariável $x_{i}, i=1, \ldots, n$, dadas por:

$$
\lambda_{1}\left(t_{i}\right)=\beta_{0} e^{-\xi_{0} x_{i}} \text { e } \lambda_{2}\left(t_{i}\right)=\beta_{1} t_{i} e^{-\xi_{1} x_{i}} .
$$


Neste caso, as funções de valor médio componentes $m_{1}\left(t_{n}\right)$ e $m_{2}\left(t_{n}\right)$ são dadas por

$$
m_{1}\left(t_{n}\right)=\beta_{0} e^{-\xi_{0} x_{n}} t_{n} \quad \text { e } \quad m_{2}\left(t_{n}\right)=\frac{\beta_{1} t_{n}^{2}}{2} e^{-\xi_{1} x_{n}}
$$

Vamos assumir que além do efeito da covariável $x_{i}$ nas funções intensidades $\lambda_{1}\left(t_{i}\right)$ e $\lambda_{2}\left(t_{i}\right)$ não existe algum efeito da covariável $x_{i}$ no parâmetro de correlação $\rho, i=1,2, \ldots, n$. Assim, de (1.18) a função de verossimilhança é dada por

$$
\begin{aligned}
L\left(\underline{\beta} \mid \underline{x}_{i} \mathfrak{D}_{\mathfrak{t}}\right) & =\prod_{i=1}^{n}\left[\lambda_{1}\left(t_{i}\right)+\lambda_{2}\left(t_{i}\right)\right]\left[1-\frac{\left(\rho W\left(t_{i}\right)\right.}{2}\right] \\
& \times \exp \left\{-\left[m_{1}\left(t_{n}\right)+m_{2}\left(t_{n}\right)-\rho\left(m_{1}\left(t_{n}\right) m_{2}\left(t_{n}\right)\right)^{1 / 2}\right]\right\}
\end{aligned}
$$

onde $W\left(t_{i}\right)$, é definido em (1.19), $m_{1}\left(t_{n}\right)$ e $m_{2}\left(t_{n}\right)$ em (3.6),respectivamente e o vetor de parâmetros deste modelo é dado por $\underline{\beta}=\left(\beta_{0}, \beta_{1}, \xi_{0}, \xi_{1}, \rho\right)$.

Modelo M4 (superposição de processos dependentes na presença de uma covariável)

A característica deste modelo é apresentar a mesma função intensidade do modelo anterior e um efeito linear na covariável $x_{i}$ no parâmetro de correlação $\rho$ dado na forma aditiva $\rho\left(x_{i}\right)=\gamma_{0}+\gamma_{1} x_{i}, i=1,2, \ldots, n$. De (1.18), a função de verossimilhança é dada por

$$
\begin{aligned}
L\left(\underline{\beta} \mid \underline{x}_{i} \mathfrak{D}_{\mathrm{t}}\right) & =\prod_{i=1}^{n}\left[\lambda_{1}\left(t_{i}\right)+\lambda_{2}\left(t_{i}\right)\right]\left[1-\frac{\left(\gamma_{0}+\gamma_{1} x_{i}\right) W\left(t_{i}\right)}{2}\right] \\
& \times \exp \left\{-\left[m_{1}\left(t_{n}\right)+m_{2}\left(t_{n}\right)-\left(\gamma_{0}+\gamma_{1} x_{n}\right)\left(m_{1}\left(t_{n}\right) m_{2}\left(t_{n}\right)\right)^{1 / 2}\right]\right\},
\end{aligned}
$$

onde $W\left(t_{i}\right)$, é definido em (1.19), $m_{1}\left(t_{n}\right)$ e $m_{2}\left(t_{n}\right)$ em (3.6), respectivamente e o vetor de parâmetros deste modelo é dado por $\underline{\beta}=\left(\beta_{0}, \beta_{1}, \xi_{0}, \xi_{1}, \gamma_{0}, \gamma_{1}\right)$.

\subsection{A Superposição de dois PPNH Polinomiais de Segundo Grau}

Consideramos agora um modelo PPNH na presença ou não de covariáveis, com $J=3$ assim (3.1); assim a função intensidade deste modelo é dada por:

$$
\lambda\left(t_{i}\right)=\lambda_{1}\left(t_{i}\right)+\lambda_{2}\left(t_{i}\right)=\left(3_{0}+\beta_{1} t_{i}\right)+3_{2} t_{i}^{2}
$$


Modelo M1 (superposição de processos independentes sem a presença de covariáveis)

Este modelo é um caso especial onde assumimos a superposição de dois PPNHs polinomiais independentes e a função intensidade deste processo é dada por (3.9); a função de valor médio deste processo de superposição é dada por:

$$
m\left(t_{n}\right)=m_{1}\left(t_{n}\right)+m_{2}\left(t_{n}\right)=\left(\beta_{0} t_{n}+\frac{\beta_{1} t_{n}^{2}}{2}\right)+\frac{\beta_{2} t_{n}^{3}}{3} .
$$

Assim, assumindo a superposição de dois PPNHs polinomiais independentes, temos $\rho=0$ e a função de verossimilhança (1.18) é reescrita por:

$$
L(\underline{\beta}, \rho)=\prod_{i=1}^{n}\left[\left(\beta_{0}+\beta_{1} t_{i}\right)+\beta_{2} t_{i}^{2}\right] \exp \left\{-\left[\left(\beta_{0} t_{n}+\frac{\beta_{1} t_{n}^{2}}{2}\right)+\frac{\beta_{2} t_{n}^{3}}{3}\right]\right\} .
$$

Denotamos o vetor de parâmetros deste modelo por $\underline{\beta}=\left(\beta_{0}, \beta_{1}, \beta_{2}\right)$.

Modelo M2 (superposição de processos dependentes sem a presença de covariáveis)

Neste modelo consideramos o caso onde a covariável $x_{i}$ não afeta a função intensidade e nem o parâmetro de correlação $\rho$, isto é, consideramos $\rho$ constante.

Considerando a função intensidade do modelo M1 em (3.9) a função de verossimilhança (1.18) com $\rho$ constante é dada por,

$$
\begin{aligned}
L(\underline{\beta}, \rho) & =\prod_{i=1}^{n}\left[\lambda_{1}\left(t_{i}\right)+\lambda_{2}\left(t_{i}\right)\right]\left[1-\frac{\rho W\left(t_{i}\right)}{2}\right] \\
& \times \exp \left\{-\left[m_{1}\left(t_{n}\right)+m_{2}\left(t_{n}\right)-\rho\left(m_{1}\left(t_{n}\right) m_{2}\left(t_{n}\right)\right)^{1 / 2}\right]\right\}
\end{aligned}
$$

onde $W\left(t_{i}\right)$ é definido em (1.19), $m_{1}\left(t_{n}\right), m_{2}\left(t_{n}\right)$ em (3.10) respectivamente. Denotamos o vetor de parâmetros deste modelo por $\underline{\beta}=\left(\beta_{0}, \beta_{1}, \beta_{2}, \rho\right)$.

Modelo M3 (superposição de processos dependentes na presença de uma covariável)

Neste modelo consideramos a situação onde a covariável $x_{i}$ afeta a função intensidade mas não afeta o parâmetro de correlação $\rho$. As funções intensidades componentes deste modelo e as funções do valor médio são os mesmos que assumimos no modelo anterior.

Neste caso o vetor de paràmetros deste modelo é dado por $\underline{\beta}=\left(\beta_{0}, \beta_{1}, \beta_{2}, \xi_{0}, \xi_{1}, \xi_{2}, \rho\right)$. 
Modelo M4 (superposição de processos dependentes na presença de uma covariável)

Neste modelo assumimos que a covariável $x_{i}$ afeta a função intensidade e a correlação $\rho$. As funções intensidades componentes deste modelo assumindo um efeito multiplicativo da covariável $x_{i}, i=1, \ldots, n$, são supostas por,

$$
\lambda_{1}\left(t_{i}\right)=\beta_{0} e^{-\xi_{0} x_{i}}+\beta_{1} t_{i} e^{-\xi_{1} x_{i}} \text { e } \lambda_{2}\left(t_{i}\right)=\beta_{2} t_{i}^{2} e^{-\xi_{2} x_{i}}
$$

Neste caso, as funções de valor médio componentes $m_{1}\left(t_{n}\right)$ e $m_{2}\left(t_{n}\right)$ são dadas por

$$
m_{1}\left(t_{n}\right)=\beta_{0} e^{-\xi_{0} x_{i}} t_{n}+\frac{\beta_{1} t_{n}^{2}}{2} e^{-\xi_{1} x_{i}} \quad \mathrm{e} m_{2}\left(t_{n}\right)=\frac{\beta_{2} t_{n}^{3}}{3} e^{-\xi_{2} x_{i}} .
$$

Também vamos assumir que além do efeito da covariável $x_{i}$ nas funções intensidades $\lambda_{1}\left(t_{i}\right)$ e $\lambda_{2}\left(t_{i}\right)$ existe um efeito da covariável $x_{i}$ no parâmetro de correlação $\rho$ dado na forma aditiva $\rho\left(x_{i}\right)=\gamma_{0}+\gamma_{1} x_{i}, i=1,2, \ldots, n$. Assim, de (1.18) a função de verossimilhança é dada por

$$
\begin{aligned}
L\left(\underline{\beta} \mid \underline{x}_{i} \mathfrak{D}_{\mathfrak{t}}\right) & =\prod_{i=1}^{n}\left[\lambda_{1}\left(t_{i}\right)+\lambda_{2}\left(t_{i}\right)\right]\left[1-\frac{\left(\gamma_{0}+\gamma_{1} x_{i}\right) W\left(t_{i}\right)}{2}\right] \\
& \times \exp \left\{-\left[m_{1}\left(t_{n}\right)+m_{2}\left(t_{n}\right)-\left(\gamma_{0}+\gamma_{1} x_{n}\right)\left(m_{1}\left(t_{n}\right) m_{2}\left(t_{n}\right)\right)^{1 / 2}\right]\right\}
\end{aligned}
$$

onde $W\left(t_{i}\right)$, é definido em (1.19), $m_{1}\left(t_{n}\right)$ e $m_{2}\left(t_{n}\right)$ dado em (3.13), respectivamente.

$\mathrm{O}$ vetor de parâmetros deste modelo é dado por $\underline{\beta}=\left(\beta_{0}, \beta_{1}, \beta_{2}, \xi_{0}, \xi_{1}, \xi_{2}, \gamma_{0}, \gamma_{1}\right)$.

\subsection{A Superposição de dois PPNH Polinomiais do Terceiro Grau}

Considerar agora o modelo PPNH na presença ou não de covariáveis, com $J=4 \mathrm{em}$ (3.1); a função intensidade para este modelo é dada por

$$
\lambda\left(t_{i}\right)=\lambda_{1}\left(t_{i}\right)+\lambda_{2}\left(t_{i}\right)=\left(\beta_{0}+\beta_{1} t+\beta_{2} t_{i}^{2}\right)+\beta_{3} t_{i}^{3},
$$

Modelo M1 (superposição de processos independentes sem a presença de covariáveis)

Assumir a superposição de dois PPNHs polinomiais independentes e a função intensidade deste processo é dada em (3.15) e a função de valor médio deste processo de 
superposição é dada por:

$$
m\left(t_{n}\right)=m_{1}\left(t_{n}\right)+m_{2}\left(t_{n}\right)=\left(\beta_{0} t_{n}+\frac{\beta_{1} t_{n}^{2}}{2}+\frac{\beta_{2} t_{n}^{3}}{3}\right)+\frac{\beta_{3} t_{n}^{4}}{4} .
$$

Assim, assumindo a superposição de dois PPNHs independentes, temos $\rho=0$ e a função de verossimilhança (1.18) é dada por:

$$
\begin{aligned}
L(\underline{\beta}, \rho)= & \prod_{i=1}^{n}\left[\left(\beta_{0}+\beta_{1} t+\beta_{2} t_{i}^{2}\right)+\beta_{3} t_{i}^{3}\right] \exp \left\{-\left[\left(\beta_{0} t_{n}+\frac{\beta_{1} t_{n}^{2}}{2}+\frac{\beta_{2} t_{n}^{3}}{3}\right)+\right.\right. \\
& \left.\left.\frac{\beta_{3} t_{n}^{4}}{4}\right]\right\} .
\end{aligned}
$$

Modelo M2 (superposição de processos dependentes sem a presença de covariáveis)

Neste modelo vamos considerar o caso onde a covariável $x_{i}$ não afeta as funções intensidades e nem o parâmetro de correlação $\rho$, isto é, consideramos $\rho$ constante.

Considerando a função intensidade do modelo em (3.15) a função de verossimilhança com $\rho$ constante é dada em (1.18).

Denotamos o vetor de parâmetros deste modelo por $\underline{\beta}=\left(\beta_{0}, \beta_{1}, \beta_{2}, \rho\right)$.

\section{Modelo M3 (superposição de processos dependentes na presença de uma covariável)}

Neste modelo vamos considerar o caso onde a covariável $x_{i}$ afeta a função intensidade mas não afeta o parâmetro de correlação $\rho$. As funções intensidades componentes deste modelo e as funções do valor médio são os mesmos que assumimos no modelo anterior.

$\mathrm{O}$ vetor de parâmetros deste modelo é dado por $\underline{\beta}=\left(\beta_{0}, \beta_{1}, \beta_{2}, \beta_{3}, \xi_{0}, \xi_{1}, \xi_{2}, \xi_{3}, \rho\right)$.

Modelo M4 (superposição de processos dependentes na presença de uma covariável)

Neste modelo assumir que a covariável $x_{i}$ afeta a função intensidade e a correlação $\rho$. As funções intensidades componentes deste modelo assumindo um efeito multiplicativo da covariável $x_{i}, i=1, \ldots, n$, são cladas por,

$$
\lambda_{1}\left(t_{i}\right)=\beta_{0} e^{-\xi_{0} x_{i}}+\beta_{1} t_{i} e^{-\xi_{1} x_{i}}+\beta_{2} t_{i}^{2} e^{-\xi_{2} x_{i}} \text { e } \lambda_{2}\left(t_{i}\right)=\beta_{3} t_{i}^{3} e^{-\xi_{3} x_{i}} .
$$

Neste caso, as funções de valor médio componentes $m_{1}(t)$ e $m_{2}(t)$ são dadas por

$$
m_{1}\left(t_{n}\right)=\beta_{0} e^{-\xi_{0} x_{i}} t_{n}+\frac{\beta_{1} t_{n}^{2}}{2} e^{-\xi_{1} x_{i}}+\frac{\beta_{2} t_{n}^{3}}{3} e^{-\xi_{2} x_{i}} \text { e } m_{2}\left(t_{n}\right)=\frac{\beta_{3} t_{n}^{4}}{4} e^{-\xi_{3} x_{i}}
$$


Também vamos assumir que além do efeito da covariável $x_{i}$ nas funções intensidades $\lambda_{1}\left(t_{i}\right)$ e $\lambda_{2}\left(t_{i}\right)$ existe um efeito da covariável $x_{i}$ no parâmetro de correlação $\rho$ dado na forma aditiva $\rho\left(x_{i}\right)=\gamma_{0}+\gamma_{1} x_{i}, i=1,2, \ldots, n$.

O vetor de parâmetros deste modelo é dado por $\underline{\beta}=\left(\beta_{0}, \beta_{1}, \beta_{2}, \beta_{3}, \xi_{0}, \xi_{1}, \xi_{2}, \xi_{3}, \gamma_{0}, \gamma_{1}\right)$.

Observar que esses modelos considerando a superposição de dois processos polinomiais pode levar a um melhor ajuste aos dados, dada a grande flexibilidade de ajuste.

Outros polinomios de ordem mais elevada também poderiam ser considerados.

\subsection{Análise Bayesiana para a Superposição de dois PPNH Polinomiais do Primeiro Grau}

\subsubsection{Modelo M2 (superposição de processos dependentes sem a presença de covariáveis)}

Para uma análise Bayesiana desse modelo, consideramos as seguintes distribuições a priori para $\beta_{0}, \beta_{1}$, e $\rho$ :

$$
\begin{aligned}
\beta_{0} & \sim \Gamma\left(a_{1}, b_{1}\right) ; \text { com } a_{1}, b_{1} \text { conhecidas } \\
\beta_{1} & \sim \Gamma\left(a_{2}, b_{2}\right) ; \text { com } a_{2}, b_{2} \text { conhecidas } \\
\rho & \sim B(c, d) ; \text { com } c, d \text { conhecidas }
\end{aligned}
$$

Existem muitas possibilidades para a escolha de distribuição priori. Neste trabalho a escolha da distribuição foi feita de acordo com o domínio do espaço paramétrico, isto é, gama para os parâmetros positivos $\left(\beta_{1}, \beta_{2}\right)$ e beta para o parâmetro $\rho$ que estão variando entre 0 e 1 . Uma pequena análise de sensitividade foi feita na escolha dos diferentes valores dos hiperparâmetros; os resultados com dados simulados mostram que as diferentes escolhas não modificam substancialmente os resultados apresentados (ver exemplo de aplicação).

Neste caso, as densidades a posteriori condicionais necessárias para o algoritmo Gibbs Sampling são dadas por:

(a) $\pi\left(\beta_{0} \mid \underline{\theta}_{\left(\beta_{0}\right)}, \underline{\nu}, \mathfrak{D}_{\mathbf{t}}\right) \propto \beta_{0}^{a_{1}-1} e^{-b_{1} \xi_{0}} \psi_{1}(\underline{\beta})$ 
onde:

$$
\begin{aligned}
\psi_{1}(\underline{\beta}) & =\exp \left\{\left(\sum_{i=1}^{n} \nu_{i 1}\right) \ln \left(\beta_{0}\right)+\sum_{i=1}^{n} \nu_{i 1} \ln \left(1-\frac{\rho A_{1 i}(\underline{\beta})}{2}\right)+\sum_{i=1}^{n} \nu_{i 2} \ln \left(1-\frac{\rho A_{2 i}(\underline{\beta})}{2}\right)\right. \\
& \left.-\beta_{0} t_{n} e^{-\xi_{0} x_{n}}+\rho\left[\beta_{0} \beta_{1} \frac{t_{n}^{3}}{2}\right]^{1 / 2}\right\}
\end{aligned}
$$

Para simplificar denotamos

$$
A_{1 i}\left(\beta_{0}, \beta_{1}\right) \text { e } A_{2 i}\left(\beta_{0}, \beta_{1}\right)
$$

por $A_{1 i}(\underline{\beta})$ e $A_{2 i}(\underline{\beta})$, onde,

(i) $A_{1 i}(\underline{\beta})=\left[\frac{m_{2}\left(t_{i}\right)}{m_{1}\left(t_{i}\right)}\right]^{1 / 2}$

(ii) $A_{2 i}(\underline{\beta})=\left[\frac{m_{1}\left(t_{i}\right)}{m_{2}\left(t_{i}\right)}\right]^{1 / 2}$

(b) $\pi\left(\beta_{1} \mid \underline{\theta}_{\left(\beta_{1}\right)}, \underline{\nu}, \mathfrak{D}_{\mathfrak{t}}\right) \propto \beta_{1}^{a_{2}-1} e^{-b_{2} \beta_{1}} \psi_{2}(\underline{\beta})$

onde:

$$
\begin{aligned}
& \begin{aligned}
\psi_{2}(\underline{\beta})= & \exp \left\{\sum_{i=1}^{n} \nu_{i 1} \ln \left(1-\frac{\rho A_{1 i}(\underline{\beta})}{2}\right)+\sum_{i=1}^{n} \nu_{i 2} \ln \left(\beta_{1}\right)+\sum_{i=1}^{n} \nu_{i 2} \ln \left(1-\frac{\rho A_{2 i}(\underline{\beta})}{2}\right)\right. \\
& \left.-\beta_{1} \frac{t_{n}^{2}}{2}\right)+\rho\left[\frac{\beta_{0} \beta_{1} t_{n}^{3}}{2}\right\}
\end{aligned} \\
& \text { (c) } \pi\left(\rho \mid \underline{\theta}_{(\rho)}, \underline{\nu}, \mathfrak{D}_{\mathfrak{t}}\right) \propto \rho^{c-1}(1-\rho)^{c-1} \psi_{3}(\underline{\beta})
\end{aligned}
$$

onde:

$$
\begin{aligned}
\psi_{3}(\underline{\beta}) & =\exp \left\{\sum_{i=1}^{n} \nu_{i 1} \ln \left(1-\frac{\rho A_{1 i}(\underline{\beta})}{2}\right)+\sum_{i=1}^{n} \nu_{i 2} \ln \left(1-\frac{\rho A_{2 i}(\underline{\beta})}{2}\right)\right. \\
& \left.+\rho\left[\frac{\beta_{0} \beta_{1}}{2} t_{n}^{3}\right]^{1 / 2}\right\}
\end{aligned}
$$

\subsubsection{Modelo M3 (superposição de processos dependentes na presença de uma covariável)}

Para o modelo M3 assumimos as seguintes distribuições a priori para $\beta_{0}, \beta_{1}, \xi_{0}, \xi_{1}$ e $\rho:$

$$
\begin{aligned}
3_{0} & \sim \Gamma\left(a_{1}, b_{1}\right) ; \operatorname{com} a_{1}, b_{1} \text { conhecidas } \\
\beta_{1} & \sim \Gamma\left(a_{2}, b_{2}\right) ; \operatorname{com} a_{2}, b_{2} \text { conhecidas } \\
\xi_{0} & \sim N\left(c_{1}, d_{1}\right) ; \operatorname{com} c_{1}, d_{1} \text { conhecidas } \\
\xi_{1} & \sim N\left(c_{2}, d_{2}\right) ; \operatorname{com} c_{2}, d_{2} \text { conhecidas } \\
\rho & \sim B\left(e_{1}, f_{1}\right) ; \operatorname{com} e_{1}, f_{1} \text { conhecidas }
\end{aligned}
$$


Também assumimos independência a priori entre os parâmetros. As distribuições condicionais necessárias para o algoritmo gibbs sampling são dadas por:

(a) $\pi\left(\beta_{0} \mid \underline{\theta}_{\left(\beta_{0}\right)}, \underline{\nu}, \mathfrak{D}_{\mathfrak{t}}\right) \propto \beta_{0}^{a_{1}-1} e^{-b_{1} \beta_{0}} \psi_{1}(\underline{\beta})$

onde:

$$
\begin{aligned}
\psi_{1}(\underline{\beta}) & =\exp \left\{\left(\sum_{i=1}^{n} \nu_{i 1}\right) \ln \left(\beta_{0}\right)+\sum_{i=1}^{n} \nu_{i 1} \ln \left(1-\frac{\rho\left(x_{i}\right) A_{1 i}(\underline{\beta})}{2}\right)+\sum_{i=1}^{n} \nu_{i 2} \ln \left(1-\frac{\rho\left(x_{i}\right) A_{2 i}(\underline{\beta})}{2}\right)\right. \\
& \left.-\beta_{0} t_{n} e^{-\xi_{0} x_{n}}+\rho\left(x_{i}\right)\left[\beta_{0} \beta_{1} \frac{t_{n}^{3}}{2} e^{-\left(\xi_{0}+\xi_{1}\right) x_{n}}\right]^{1 / 2}\right\}
\end{aligned}
$$

Para simplificar denotamos

$$
A_{1 i}\left(\beta_{0}, \beta_{1}\right) \text { e } A_{2 i}\left(\beta_{0}, \beta_{1}\right)
$$

por $A_{1 i}(\underline{\beta})$ e $A_{2 i}(\underline{\beta})$, onde,

(i) $A_{1 i}(\underline{\beta})=\left[\frac{m_{2}\left(t_{i}\right)}{m_{1}\left(t_{i}\right)}\right]^{1 / 2}=\left[\frac{\beta_{0} t_{i} e^{-\xi_{1} x_{i}}}{2 \beta_{0} e^{-\xi_{0} x_{i}}}\right]^{1 / 2}$

(ii) $A_{2 i}(\underline{\beta})=\left[\frac{m_{1}\left(t_{i}\right)}{m_{2}\left(t_{i}\right)}\right]^{1 / 2}=\left[\frac{2 \beta_{0} e^{-\xi_{0} x_{i}}}{\beta_{1} t_{i} e^{-\xi_{1} x_{i}}}\right]^{1 / 2}$

(b) $\pi\left(\beta_{1} \mid \underline{\theta}_{\left(\beta_{1}\right)}, \underline{\nu}, \mathfrak{D}_{\mathfrak{t}}\right) \propto \beta_{1}^{a_{2}-1} e^{-b_{2} \beta_{1}} \psi_{2}(\underline{\beta})$

onde:

$$
\begin{gathered}
\begin{aligned}
& \psi_{2}(\underline{\beta})=\exp \left\{\sum_{i=1}^{n} \nu_{i 1} \ln \left(1-\frac{\rho A_{1 i}(\underline{\beta})}{2}\right)+\sum_{i=1}^{n} \nu_{i 2} \ln \left(\beta_{1}\right)+\sum_{i=1}^{n} \nu_{i 2} \ln \left(1-\frac{\rho A_{2 i}(\underline{\beta})}{2}\right)\right. \\
&-\beta_{1} \frac{t_{n}^{2}}{2} e^{-\xi_{1} x_{n}}+\rho\left(x_{n}\right)\left[\frac{\beta_{0} \beta_{1} t_{n}^{3}}{2} e^{-\left(\xi_{0}+\xi_{1}\right) x_{n}}\right\} \\
& \text { (c) } \pi\left(\xi_{0} \mid \underline{\theta}_{\left(\xi_{0}\right)}, \underline{\nu}, \mathfrak{D}_{\mathfrak{t}}\right) \propto \exp \left\{-\frac{1}{2 d_{1}^{2}}\left(\xi_{0}-c_{1}\right)^{2}\right\} \dot{\psi}_{3}(\underline{\beta})
\end{aligned}
\end{gathered}
$$

onde:

$$
\begin{aligned}
& \dot{\psi}_{3}(\underline{\beta})=\exp \left\{-\left(\sum_{i=1}^{n} \nu_{i 1} x_{i}\right) \xi_{0}+\sum_{i=1}^{n} \nu_{i 1} \ln \left(1-\frac{\rho A_{1 i}(\underline{\beta})}{2}\right)+\sum_{i=1}^{n} \nu_{i 2} \ln \left(1-\frac{\rho A_{2 i}(\underline{\beta})}{2}\right)\right. \\
&\left.-3_{0} t_{n} e^{-\xi_{0} x_{n}}+\rho\left(x_{n}\right)\left[\frac{\beta_{0} \beta_{1}}{2} t_{n}^{3} e^{-\left(\xi_{0}+\xi_{1}\right) \cdot x_{n}}\right]^{1 / 2}\right\} \\
& \text { (d) } \pi\left(\xi_{1} \mid \underline{\theta}_{\left(\xi_{1}\right)}, \underline{\nu}, \mathfrak{D}_{\mathfrak{t}}\right) \propto \exp \left\{-\frac{1}{2 d_{2}^{2}}\left(\xi_{1}-c_{2}\right)^{2}\right\} \psi_{4}(\underline{\beta})
\end{aligned}
$$


onde:

$$
\begin{aligned}
\psi_{4}(\underline{\beta}) & =\exp \left\{\sum_{i=1}^{n} \nu_{i 1} \ln \left(1-\frac{\rho A_{1 i}(\underline{\beta})}{2}\right)-\left(\sum_{i=1}^{n} \nu_{i 2} x_{i}\right) \xi_{1}+\sum_{i=1}^{n} \nu_{i 2} \ln \left(1-\frac{\rho A_{2 i}(\underline{\beta})}{2}\right)\right. \\
& \left.-\frac{\beta_{1} t_{n}^{2}}{2} e^{-\xi_{1} x_{n}}+\rho\left(x_{n}\right)\left[\frac{\beta_{0} \beta_{1}}{2} t_{n}^{3} e^{-\left(\xi_{0}+\xi_{1}\right) x_{n}}\right]^{1 / 2}\right\}
\end{aligned}
$$

(e) $\pi\left(\rho \mid \underline{\theta}_{(\rho)}, \underline{\nu}, \mathfrak{D}_{\mathfrak{t}}\right) \propto \rho^{c_{1}-1}(1-\rho)^{d_{1}-1} \psi_{5}(\underline{\beta})$

onde:

$$
\begin{aligned}
\psi_{5}(\underline{\beta}) & =\exp \left\{\sum _ { i = 1 } ^ { n } \nu _ { i 1 } \operatorname { l n } \left(1-\frac{\rho\left(x_{i}\right)}{2} A_{1 i}(\underline{\beta})+\sum_{i=1}^{n} \nu_{i 2} \ln \left(1-\frac{\rho\left(x_{i}\right)}{2} A_{2 i}(\underline{\beta})\right)\right.\right. \\
& \left.+\rho\left(x_{n}\right)\left[\frac{\beta_{0} \beta_{1}}{2} t_{n}^{3} e^{-\left(\xi_{0}+\xi_{1}\right) x_{n}}\right]^{1 / 2}\right\}
\end{aligned}
$$

\subsubsection{Modelo M4 (superposição de processos dependentes na presença de uma covariável)}

Para o modelo M4 assumimos as seguintes distribuições a priori para $\beta_{0}, \beta_{1}, \xi_{0}, \xi_{1}, \gamma_{0}$ e $\gamma_{1}$ :

$$
\begin{aligned}
\beta_{0} & \sim \Gamma\left(a_{1}, b_{1}\right) ; \operatorname{com} a_{1}, b_{1} \text { conhecidas } \\
\beta_{1} & \sim \Gamma\left(a_{2}, b_{2}\right) ; \operatorname{com} a_{2}, b_{2} \text { conhecidas } \\
\xi_{0} & \sim N\left(c_{0}, d_{0}\right) ; \operatorname{com} c_{0}, d_{0} \text { conhecidas } \\
\xi_{1} & \sim N\left(c_{1}, d_{1}\right) ; \operatorname{com} c_{1}, d_{1} \text { conhecidas } \\
\gamma_{0} & \sim B\left(e_{0}, f_{0}\right) ; \text { com } e_{0}, f_{0} \text { conhecidas } \\
\gamma_{1} & \sim B\left(e_{1}, f_{1}\right) ; \text { com } e_{1}, f_{1} \text { conhecidas }
\end{aligned}
$$

As distribuições condicionais para o amostrador de Gibbs sampling são dadas por:

(a) $\pi\left(\beta_{0} \mid \underline{\theta}_{\left(3_{0}\right)}, \underline{\nu}, \mathfrak{D}_{\mathfrak{t}}\right) \propto \beta_{0}^{a_{1}-1} e^{-b_{1} 3_{0}} \psi_{1}(\underline{\beta})$

onde:

$$
\begin{aligned}
& \dot{\nu}_{1}(\underline{\beta})=\exp \left\{\left(\sum_{i=1}^{n} \nu_{i 1}\right) \ln \left(\beta_{0}\right)+\sum_{i=1}^{n} \nu_{i 1} \ln \left(1-\frac{\rho\left(x_{i}\right) A_{1 i}(\underline{\beta})}{2}\right)+\sum_{i=1}^{n} \nu_{i 2} \ln \left(1-\frac{\rho\left(x_{i}\right) A_{2 i}(\underline{\beta})}{2}\right)\right. \\
&\left.-\beta_{0} t_{n} e^{-\xi_{0} \cdot x_{n}}+\rho\left(x_{n}\right)\left[\beta_{0} \beta_{1} \frac{t_{n}^{3}}{2} e^{-\left(\xi_{0}+\xi_{1}\right) x_{n}}\right]^{1 / 2}\right\} \\
& \text { (b) } \pi\left(\beta_{1} \mid \underline{\theta}_{\left(\xi_{1}\right)}, \underline{\nu}, \mathfrak{D}_{\mathfrak{t}}\right) \propto \beta_{1}^{a a_{2}-1} e^{-b_{2} \beta_{1}} \psi_{2}(\underline{\beta})
\end{aligned}
$$


onde:

$$
\begin{aligned}
& \begin{aligned}
\psi_{2}(\underline{\beta}) & =\exp \left\{\sum_{i=1}^{n} \nu_{i 1} \ln \left(1-\frac{\rho A_{1 i}(\underline{\beta})}{2}\right)+\sum_{i=1}^{n} \nu_{i 2} \ln \left(\beta_{1}\right)+\sum_{i=1}^{n} \nu_{i 2} \ln \left(1-\frac{\rho A_{2 i}(\underline{\beta})}{2}\right)\right. \\
& -\beta_{1} \frac{t_{n}^{2}}{2} e^{-\xi_{1} x_{n}}+\rho\left[\frac{\beta_{0} \beta_{1} t_{n}^{3}}{2} e^{-\left(\xi_{0}+\xi_{1}\right) x_{n}}\right\}
\end{aligned} \\
& \text { (c) } \pi\left(\xi_{0} \mid \underline{\theta}_{\left(\xi_{0}\right)}, \underline{\nu}, \mathfrak{D}_{\mathrm{t}}\right) \propto \exp \left\{-\frac{1}{2 d_{0}^{2}}\left(\xi_{0}-c_{0}\right)^{2}\right\} \psi_{3}(\underline{\beta})
\end{aligned}
$$

onde:

$$
\begin{aligned}
& \begin{aligned}
\psi_{3}(\underline{\beta}) & =\exp \left\{-\left(\sum_{i=1}^{n} \nu_{i 1} x_{i}\right) \xi_{0}+\sum_{i=1}^{n} \nu_{i 1} \ln \left(1-\frac{\rho A_{1 i}(\underline{\beta})}{2}\right)+\sum_{i=1}^{n} \nu_{i 2} \ln \left(1-\frac{\rho A_{2 i}(\underline{\beta})}{2}\right)\right. \\
& \left.-\beta_{0} t_{n} e^{-\xi_{0} x_{n}}+\rho\left[\frac{\beta_{0} \beta_{1}}{2} t_{n}^{3} e^{-\left(\xi_{0}+\xi_{1}\right) x_{n}}\right]^{1 / 2}\right\}
\end{aligned} \\
& \text { (d) } \pi\left(\xi_{1} \mid \underline{\theta}_{\left(\xi_{1}\right)}, \underline{\nu}, \mathfrak{D}_{\mathfrak{t}}\right) \propto \exp \left\{-\frac{1}{2 d_{1}^{2}}\left(\xi_{1}-c_{1}\right)^{2}\right\} \psi_{4}(\underline{\beta})
\end{aligned}
$$

onde:

$$
\begin{aligned}
\psi_{4}(\underline{\beta}) & =\exp \left\{\sum_{i=1}^{n} \nu_{i 1} \ln \left(1-\frac{\rho A_{1 i}(\underline{\beta})}{2}\right)-\left(\sum_{i=1}^{n} \nu_{i 2} x_{i}\right) \xi_{1}+\sum_{i=1}^{n} \nu_{i 2} \ln \left(1-\frac{\rho A_{2 i}(\underline{\beta})}{2}\right)\right. \\
& \left.-\frac{\beta_{1} t_{n}^{2}}{2} e^{-\xi_{1} x_{n}}+\rho\left[\frac{\beta_{0} \beta_{1}}{2} t_{n}^{3} e^{-\left(\xi_{0}+\xi_{1}\right) x_{n}}\right]^{1 / 2}\right\}
\end{aligned}
$$

(e) $\pi\left(\gamma_{0} \mid \underline{\theta}_{\left(\gamma_{0}\right)}, \underline{\nu}, \mathfrak{D}_{\mathrm{t}}\right) \propto \gamma_{0}^{c_{0}-1}\left(1-\gamma_{0}\right)^{d_{0}-1} \psi_{5}(\underline{\beta})$

onde:

$$
\begin{aligned}
& \begin{aligned}
\psi_{5}(\underline{\beta}) & =\exp \left\{\sum _ { i = 1 } ^ { n } \nu _ { i 1 } \operatorname { l n } \left(1-\frac{\left(\gamma_{0}+\gamma_{1} x_{i}\right)}{2} A_{1 i}(\underline{\beta})+\sum_{i=1}^{n} \nu_{i 2} \ln \left(1-\frac{\left(\gamma_{0}+\gamma_{1} x_{i}\right)}{2} A_{2 i}(\underline{\beta})\right)\right.\right. \\
& \left.+\left(\gamma_{0}+\gamma_{1} x_{i}\right)\left[\frac{\beta_{0} \beta_{1}}{2} t_{n}^{3} e^{-\left(\xi_{0}+\xi_{1}\right) x_{n}}\right]^{1 / 2}\right\}
\end{aligned} \\
& \text { (f) } \pi\left(\xi_{1} \mid \underline{\theta}_{\left(\xi_{1}\right)}, \underline{\nu}, \mathfrak{D}_{\mathrm{t}}\right) \propto \xi_{1}^{c_{2}-1}\left(1-\xi_{1}\right)^{d_{2}-1} \psi_{6}(\underline{\beta})
\end{aligned}
$$

onde:

$$
\begin{aligned}
\psi_{6}(\underline{\beta}) & =\exp \left\{\sum _ { i = 1 } ^ { n } \nu _ { i 1 } \operatorname { l n } \left(1-\frac{\left(\gamma_{0}+\gamma_{1} x_{i}\right)}{2} A_{1 i}(\underline{\beta})+\sum_{i=1}^{n} \nu_{i 2} \ln \left(1-\frac{\left(\gamma_{0}+\gamma_{1} x_{i}\right)}{2} A_{2 i}(\underline{\beta})\right)\right.\right. \\
& \left.+\left(\gamma_{0}+\gamma_{1} x_{i}\right)\left[\frac{3_{0} \beta_{1}}{2} t_{n}^{3} e^{-\left(\varepsilon_{0}+\xi_{1}\right) x_{n}}\right]^{1 / 2}\right\}
\end{aligned}
$$

Observar que as distribuições condicionais a posteriori cladlas em (3.23) não possuem formas conhecidas, de modo que faremos uso do algoritmo de Metropolis-Hastings (ver, Chib e Greenberg, 1995) para gerar amostras a posteriori para $\beta_{0}, \zeta_{1}, \xi_{0}, \xi_{1}, \gamma_{0}$ e $\gamma_{1}$.

De forma similar as clistribuições condicionais a posteriori foram obtidas para a análise dos outros modelos, assumindo distribuições a priori convenientes para cada caso. 


\subsection{Aplicações Numéricas}

Neste seção, apresentamos aplicações dos modelos apresentados nas seções anteriores.

\subsubsection{Exemplo 1}

Na tabela 3.1, apresentamos um conjunto de dados simulados a partir de superposição de PPNHs polinomiais independentes com função intensidade dada por

$$
\lambda_{1}\left(t_{i}\right)=\beta_{0} e^{-\xi_{0} x_{i}}+\beta_{1} t_{i} e^{-\xi_{1} x_{i}}
$$

Tabela 3.1: Dados gerados a partir de um processo PPNH.

\begin{tabular}{c|c|c|c|c|c|c|c|c|c}
\hline \hline$t_{i}$ & $x_{i}$ & $t_{i}$ & $x_{i}$ & $t_{i}$ & $x_{i}$ & $t_{i}$ & $x_{i}$ & $t_{i}$ & $x_{i}$ \\
\hline 10,6306 & 1 & 86,1097 & 2 & 165,4108 & 1 & 235,6580 & 2 & 343,3536 & 1 \\
11,2891 & 1 & 96,2841 & 2 & 165,9430 & 1 & 246,0373 & 1 & 349,9357 & 1 \\
15,6073 & 2 & 96,3057 & 2 & 173,0731 & 1 & 249,2204 & 2 & 359,3489 & 1 \\
20,9628 & 1 & 102,7717 & 1 & 175,6523 & 2 & 252,8160 & 2 & 362,0208 & 1 \\
25,1134 & 2 & 137,3521 & 2 & 199,1072 & 2 & 255,2921 & 2 & 363,7467 & 2 \\
25,1954 & 1 & 148,1138 & 1 & 203,0930 & 1 & 271,1487 & 1 & 370,1592 & 1 \\
38,8020 & 1 & 149,3906 & 1 & 216,6014 & 1 & 290,5067 & 2 & 381,4956 & 1 \\
56,1137 & 2 & 157,9336 & 1 & 220,7397 & 1 & 305,3189 & 1 & 382,5202 & 1 \\
74,9975 & 2 & 159,3679 & 1 & 228,7703 & 1 & 338,0622 & 1 & 390,5408 & 1 \\
85,5960 & 2 & 159,5329 & 1 & 232,1089 & 2 & 343,3429 & 1 & 398,8943 & 1 \\
\hline \hline
\end{tabular}

para os valores fixados $\beta_{0}=2, \beta_{1}=1, \xi_{0}=3$ e $\xi_{1}=5 ; 50$ observações foram geradas considerando uma covariável com valores 1 e 2 . Observar que na geração desses dados assumimos $\rho=0,70$.

Os resultados da análise dos modelos foram obtidos usando métodos MCMC implementado no software WINBUGS ( Spiegelhalter et al., 1999) após descartar as 4000 primeiras observações (burn-in).

Modelo M2 (superposição de processos dependentes sem a presença de covariáveis)

A seguir desenvolvemos uma análise Bayesiana assumindo os vários modelos propostos previamente. Para o modelo M2 assumimos uma superposição de dois PPNHs polinomiais dependentes onde a covariável $x$ não afeta as intensidades e a correlação $\rho$. 
Considerando as distribuições a priori dadas para o modelo M2 dadas em 3.21; fixamos alguns valores para os hiperparâmetros (Vrs Hip), os sumários a posteriori são dados na Tabela 3.2, onde estão apresentadas características tais como média a posteriori, desvio-padrão (DP) e intervalos de credibilidade de 95 \% para os parâmetros de interesse. Também apresentamos o fator de escala de redução $\hat{R}$ (Gelman e Rubin, 1992) para os parâmetros $\beta_{0}, \beta_{1}, \rho$ do modelo M2. Com valores diferentes dos hiperparâmetros, as médias a posteriori são próximas para cada parâmetros do modelo, variando apenas os erros-padrão e os resultados para as cadeias geradas foram satisfatórios, e isso pode ser visto graficamente na Figura 3.1 que apresenta o traço e a densidade dos parâmetros de interesse.

Tabela 3.2: Resumos a posteriori para o modelo M2.

\begin{tabular}{c|c|c|c|c|c}
\hline Parâmetro & Vrs. Hip & Média & D.P & Interv. de Credibilidade & $\hat{R}$ \\
\hline \hline \multirow{4}{*}{$\beta_{0}$} & $\Gamma(12 ; 6)$ & 1,9654 & 0,5778 & $(0,9916 ; 3,243)$ & 1,004 \\
\cline { 2 - 6 } & $\Gamma(36 ; 18)$ & 1,981 & 0,3341 & $(1,381 ; 2,689)$ & 1,009 \\
\cline { 2 - 6 } & $\Gamma(100 ; 50)$ & 1,992 & 0,2002 & $(1,618 ; 2,404)$ & 1,005 \\
\hline \multirow{3}{*}{$\beta_{1}$} & $\Gamma(6 ; 3)$ & 1,059 & 0,01395 & $(1,032 ; 1,087)$ & 1,001 \\
\cline { 2 - 6 } & $\Gamma(0,001 ; 0,001)$ & 1,059 & 0,01294 & $(1,034 ; 1,084)$ & 1,017 \\
\cline { 2 - 6 } & $\Gamma(1 ; 0,001)$ & 1,059 & 0,01292 & $(1,033 ; 1,084)$ & 1,003 \\
\hline \multirow{4}{*}{$\rho$} & $\Gamma(42 ; 18)$ & 0,6826 & 0,05887 & $(0,5627 ; 0,7923)$ & 1,024 \\
\cline { 2 - 6 } & $\Gamma(35 ; 15)$ & 0,678 & 0,0644 & $(0,5454 ; 0,7982)$ & 1,026 \\
\cline { 2 - 6 } & $\Gamma(28,12)$ & 0,6724 & 0,07166 & $(0,5265 ; 0,8062)$ & 1,006 \\
\hline \hline
\end{tabular}

Também observamos convergência do algoritmo a partir dos fatores de escala de redução $\hat{R}$ introduzidos por Gelman e Rubin (1992). As estimativas Bayesianas para os parâmetros $\beta_{0}, \beta_{1}$ e $\rho$ estão próximos dos valores usados para simulação dos dados.

\section{Modelo M3 (superposição de processos polinomiais dependentes)}

Neste caso, vamos considerar a superposição PPNH polinomiais, dependentes onde a covariável afeta as intensidacles individuais para cada componente, mas não afeta o parâmetro de correlação $\rho$.

Considerando as distribuições a priori do modelo M3 dadas em 3.21, na Tabela 3.3, apresentamos os resumos a posteriori correspondentes aos parâmetros $\beta_{0}, \beta_{1}, \xi_{0}, \xi_{1}$ e $\rho$, onde fixamos cliferentes valores para os hiperparâmetros. Nesta Tabela estão apresentadas características tais como média a posteriori, desvio-padrão (DP) e intervalos de 

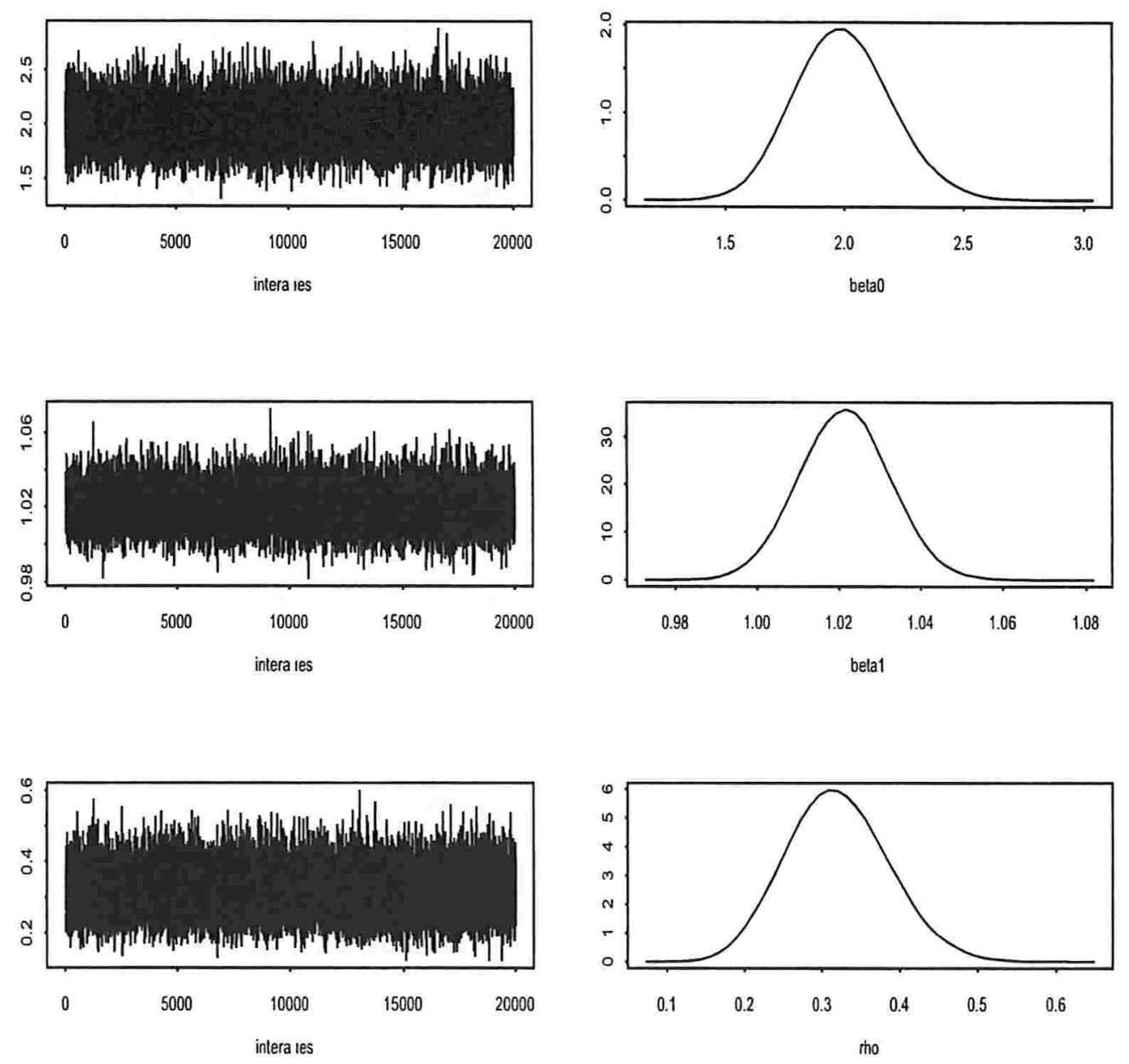

Figura 3.1: O traço e a Densidades a posteriori de $\beta_{0}, \beta_{1}$ e $\rho$.

credibilidade de $95 \%$ para os parâmetros de interesse. Também é mostrado o fator de escala de redução $\hat{R}$ para os parâmetros $\beta_{0}, \beta_{1}, \xi_{0}, \xi_{1}$ e $\rho$ do modelo M3. Com valores diferentes dos hiperparâmetros as médias a posteriori são próximas para cada parâmetros do modelo, variando apenas os erros-padrão e os resultados para as cadeias geradas foram satisfatórios, e isso pode ser visto graficamente na Figura 3.2 que apresenta o traço e a densidade dos parâmetros de interesse. Também temos $\hat{R}$ menores do que 1,1 para todos os casos, o que indica convergência do algoritmo. 
Tabela 3.3: Resumos a posteriori para o modelo M3.

\begin{tabular}{c|c|c|c|c|c}
\hline Parâmetro & Vrs. Hip & Média & D.P & Interv. de Credibilidade & $\hat{R}$ \\
\hline \hline \multirow{6}{*}{$\beta_{0}$} & $\Gamma(32,16)$ & 1,977 & 0,3497 & $(1,352 ; 2,72)$ & 1,009 \\
\cline { 2 - 6 } & $\Gamma(400,200)$ & 1,998 & 0,1003 & $(1,807 ; 2,2)$ & 1,006 \\
\cline { 2 - 6 } & $\Gamma(36 ; 18)$ & 1,98 & 0,3281 & $(1,39 ; 2,67)$ & 1,001 \\
\hline \multirow{5}{*}{$\beta_{1}$} & $\Gamma(16 ; 8)$ & 1,008 & 0,01057 & $(0,9876 ; 1,029)$ & 1,001 \\
\cline { 2 - 6 } & $\Gamma(0,001 ; 0,001)$ & 1,015 & 0,01187 & $(0,9922 ; 1,039)$ & 1,012 \\
\cline { 2 - 6 } & $\Gamma(0,01 ; 0,01)$ & 1,015 & 0,01303 & $(0,991 ; 1,042)$ & 1,026 \\
\hline \multirow{5}{*}{$\xi_{0}$} & $N(5 ; 100)$ & 4,998 & 0,09932 & $(4,802 ; 5,191)$ & 1,005 \\
\cline { 2 - 6 } & $N(5 ; 10)$ & 4,991 & 0,3161 & $(4,371 ; 5,613)$ & 1,039 \\
\cline { 2 - 6 } & $N(5,5)$ & 4,994 & 0,4356 & $(4,146 ; 5,848)$ & 1,005 \\
\hline \multirow{5}{*}{$\xi_{1}$} & $N(3 ; 25)$ & 3,008 & 0,1995 & $(2,621 ; 3,404)$ & 1,011 \\
\cline { 2 - 6 } & $N(3 ; 5)$ & 3,012 & 0,4393 & $(2,154 ; 3,878)$ & 1,002 \\
\cline { 2 - 6 } & $N(3,10)$ & 3,008 & 0,3113 & $(2,397 ; 3,619)$ & 1,081 \\
\hline \multirow{5}{*}{$\rho$} & $B(36 ; 16)$ & 0,693 & 0,06326 & $(0,5629 ; 0,8095)$ & 1,005 \\
\cline { 2 - 6 } & $B(21 ; 9)$ & 0,7015 & 0,08169 & $(0,5302 ; 0,8477)$ & 1,019 \\
\cline { 2 - 6 } & $B(63,27)$ & 0,6998 & 0,04808 & $(0,6025 ; 0,7896)$ & 1,001 \\
\hline
\end{tabular}

Modelo M4 (superposição de processos dependentes na presença de uma covariável)

Neste caso, vamos considerar a superposição de PPNH polinomiais, dependentes onde a covariável afeta as intensidades individuais para cada componente e o parâmetro de correlação $\rho$.

Considerando as distribuições a priori do modelo M4 dadas em 3.22, na Tabela 3.4, apresentamos os resumos a posteriori correspondentes aos parâmetros $\beta_{0}, \beta_{1}, \xi_{0}, \xi_{1}, \gamma_{0}$ e $\gamma_{1}$, onde fixamos diferentes valores para os hiperparâmetros. Nesta Tabela estão apresentadas características tais como média a posteriori, desvio-padrão (DP) e intervalos de credibilidade de $95 \%$ para os parâmetros de interesse. Também é mostrado o fator de escala de redução $\hat{R}$ para os parâmetros $\beta_{0}, \beta_{1}, \xi_{0}, \xi_{1}, \gamma_{0}$ e $\gamma_{1}$ do modelo M3. Com valores diferentes dos hiperparâmetros as médias a posteriori são próximas para cada parâmetros do modelo, variando apenas os erros-padrão e os resultados para as cadeias geradas foram satisfatórios, e isso pode ser visto graficamente na Figura 3.3 que apresenta o traço e a clensidade dos parâmetros de interesse. Também temos $\hat{R}$ menores do que 1,1 para todos os casos, o que indica convergência do algoritmo. 


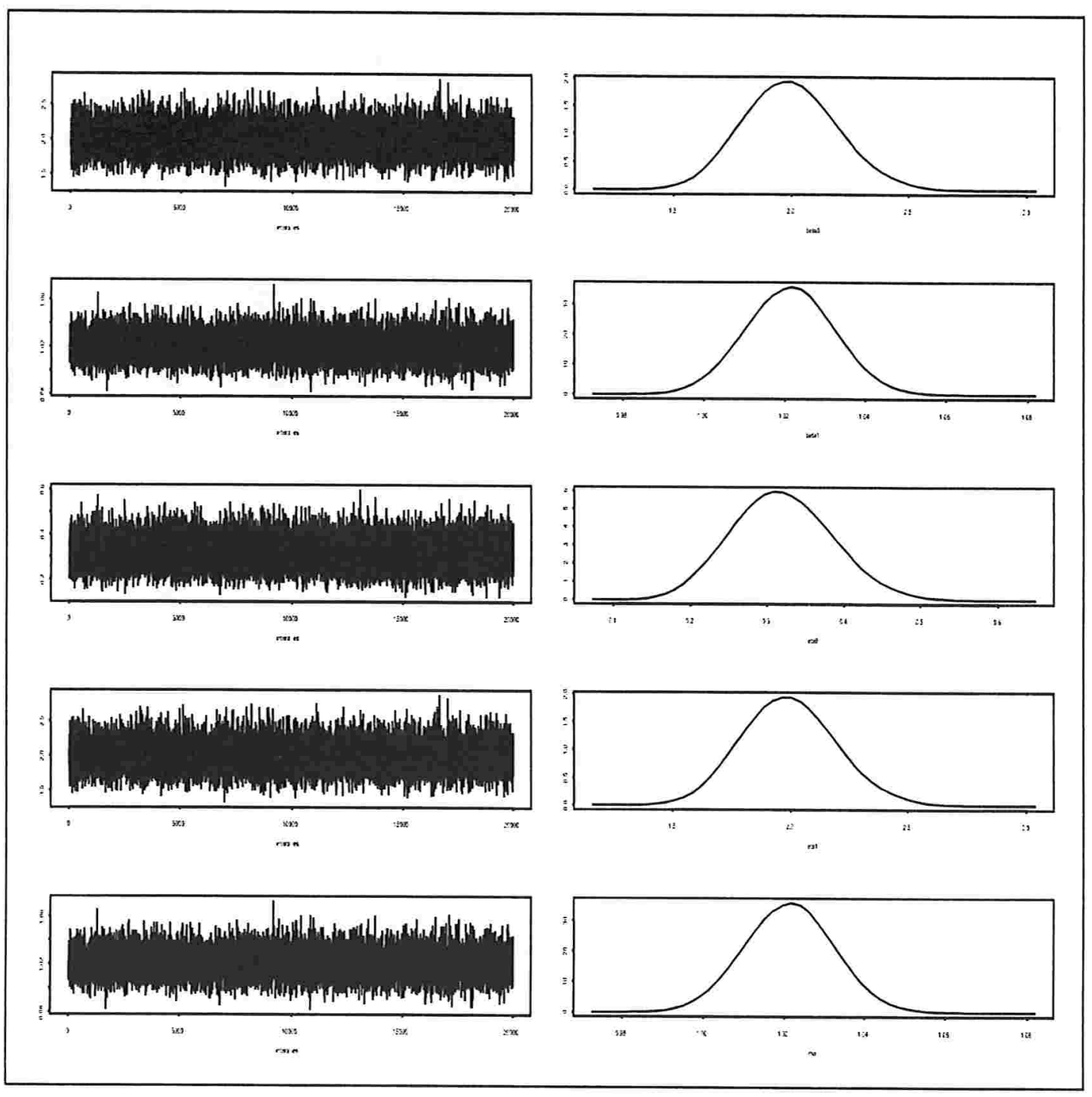

Figura 3.2: O traço e a Densidades a posteriori de $\beta_{0}, \beta_{1}, \xi_{0}, \xi_{1}$ e $\rho$

\subsubsection{Exemplo 2}

Na Tabela 3.5 apresentamos um conjunto de dados simulados a partir de superposição de PPNH polinomiais com função intensidade

$$
\lambda\left(t_{i}\right)=\beta_{0} e^{-\xi_{0} x_{i}}+\beta_{1} t_{i} e^{-\xi_{1} x_{i}}+\beta_{2} t_{i}^{2} e^{-\xi_{2} x_{i}}
$$

para os valores fixados $\beta_{0}=2, \beta_{1}=1, \beta_{2}=0,0005, \xi_{0}=10, \xi_{1}=0,04$ e $\xi_{2}=6 ; 50$ observações foram geradas considerando uma covariável com valores 1 e 2 . Observar que na geração desse dados assumimos $\rho=0,60$.

Os resultados da análise dos modelos foram obtido atráves de métodos MCMC implementado no software WINBUGS ( Spiegelhalter et al., 1999) após descartar as 4000 
Tabela 3.4: Resumos a posteriori para o modelo M4.

\begin{tabular}{c|c|c|c|c|c}
\hline Parâmetro & Vrs. Hip & Média & D.P & Interv. de Credibilidade & $\hat{R}$ \\
\hline \hline \multirow{6}{*}{$\beta_{0}$} & $\Gamma(32,16)$ & 1,965 & 0,3431 & $(1,348 ; 2,688)$ & 1,001 \\
\cline { 2 - 6 } & $\Gamma(400,200)$ & 1,997 & 0,09966 & $(1,806 ; 2,197)$ & 1,001 \\
\cline { 2 - 6 } & $\Gamma(36 ; 18)$ & 1,971 & 0,3251 & $(1,381 ; 2,653)$ & 1,002 \\
\hline \multirow{5}{*}{$\beta_{1}$} & $\Gamma(16 ; 8)$ & 1,008 & 0,01057 & $(0,9876 ; 1,029)$ & 1,001 \\
\cline { 2 - 6 } & $\Gamma(0,001 ; 0,001)$ & 1,008 & 0,01109 & $(0,9865 ; 1,030)$ & 1,022 \\
\cline { 2 - 6 } & $\Gamma(0,01 ; 0,01)$ & 1,010 & 0,01272 & $(0,9862 ; 1,036)$ & 1,004 \\
\hline \multirow{3}{*}{$\xi_{0}$} & $N(5 ; 100)$ & 4,998 & 0,09932 & $(4,802 ; 5,191)$ & 1,005 \\
\cline { 2 - 6 } & $N(5 ; 10)$ & 4,99 & 0,3155 & $(4,372 ; 5,611)$ & 1,001 \\
\cline { 2 - 6 }$\xi_{1}$ & $N(5,5)$ & 4,984 & 0,4444 & $(4,115 ; 5,86)$ & 1,025 \\
\cline { 2 - 6 } & $N(3 ; 25)$ & 3,008 & 0,1995 & $(2,621 ; 3,404)$ & 1,011 \\
\cline { 2 - 6 } & $N(3 ; 10)$ & 3,017 & 0,3181 & $(2,399 ; 3,645)$ & 1,043 \\
\hline \multirow{2}{*}{$\gamma_{0}$} & $N(3,5)$ & 3,021 & 0,4455 & $(2,133 ; 3,886)$ & 1,011 \\
\cline { 2 - 6 } & $B(12 ; 28)$ & 0,3043 & 0,072 & $(0,1733 ; 0,45833)$ & 1,006 \\
\cline { 2 - 6 } & $B(30 ; 70)$ & 0,3009 & 0,04574 & $(0,2156 ; 0,3941)$ & 1,003 \\
\hline \multirow{2}{*}{$\gamma_{1}$} & $B(6,14)$ & 0,3035 & 0,09938 & $(0,1291 ; 0,5115)$ & 1,001 \\
\cline { 2 - 6 } & $B(14 ; 56)$ & 0,2018 & 0,0477 & $(0,1173 ; 0,3036)$ & 1,009 \\
\cline { 2 - 6 } & $B(12 ; 48)$ & 0,2014 & 0,05142 & $(0,1104 ; 0,3104)$ & 1,001 \\
\cline { 2 - 6 } & $B(7,23)$ & 0,235 & 0,07581 & $(0,1048 ; 0,3994)$ & 1,003 \\
\hline \hline
\end{tabular}

primeiras observações (burn-in).

Tabela 3.5: Dados gerados a partir de um processo PPNH.

\begin{tabular}{c|c|c|c|c|c|c|c|c|c}
\hline \hline$t_{i}$ & $x_{i}$ & $t_{i}$ & $x_{i}$ & $t_{i}$ & $x_{i}$ & $t_{i}$ & $x_{i}$ & $t_{i}$ & $x_{i}$ \\
\hline 16,8020 & 1 & 122,8344 & 1 & 217,1556 & 1 & 279,4498 & 1 & 334,2854 & 1 \\
30,4973 & 1 & 127,7346 & 1 & 226,9226 & 2 & 285,1199 & 2 & 338,7823 & 2 \\
35,3689 & 1 & 138,5892 & 2 & 239,3495 & 2 & 292,6699 & 1 & 339,0948 & 2 \\
44,8513 & 1 & 154,1542 & 1 & 242,5827 & 1 & 294,1804 & 1 & 340,9788 & 2 \\
50,2903 & 1 & 163,9526 & 2 & 251,6214 & 1 & 295,2527 & 1 & 342,7167 & 1 \\
55,7758 & 1 & 193,4524 & 1 & 260,7475 & 2 & 301,4230 & 1 & 349,6443 & 1 \\
58,6047 & 1 & 199,2737 & 2 & 265,0749 & 2 & 307,9631 & 2 & 355,4436 & 2 \\
59,2910 & 1 & 203,1503 & 1 & 267,0238 & 1 & 313,5644 & 1 & 363,6157 & 2 \\
79,7070 & 1 & 212,1710 & 2 & 267,5278 & 1 & 320,8508 & 1 & 392,3553 & 1 \\
112,7705 & 1 & 212,6769 & 1 & 274,4078 & 1 & 323,1998 & 1 & 397,8285 & 2 \\
\hline
\end{tabular}

A seguir vamos apresentar os resultados obtidos considerando os modelos introduzidos acima. 


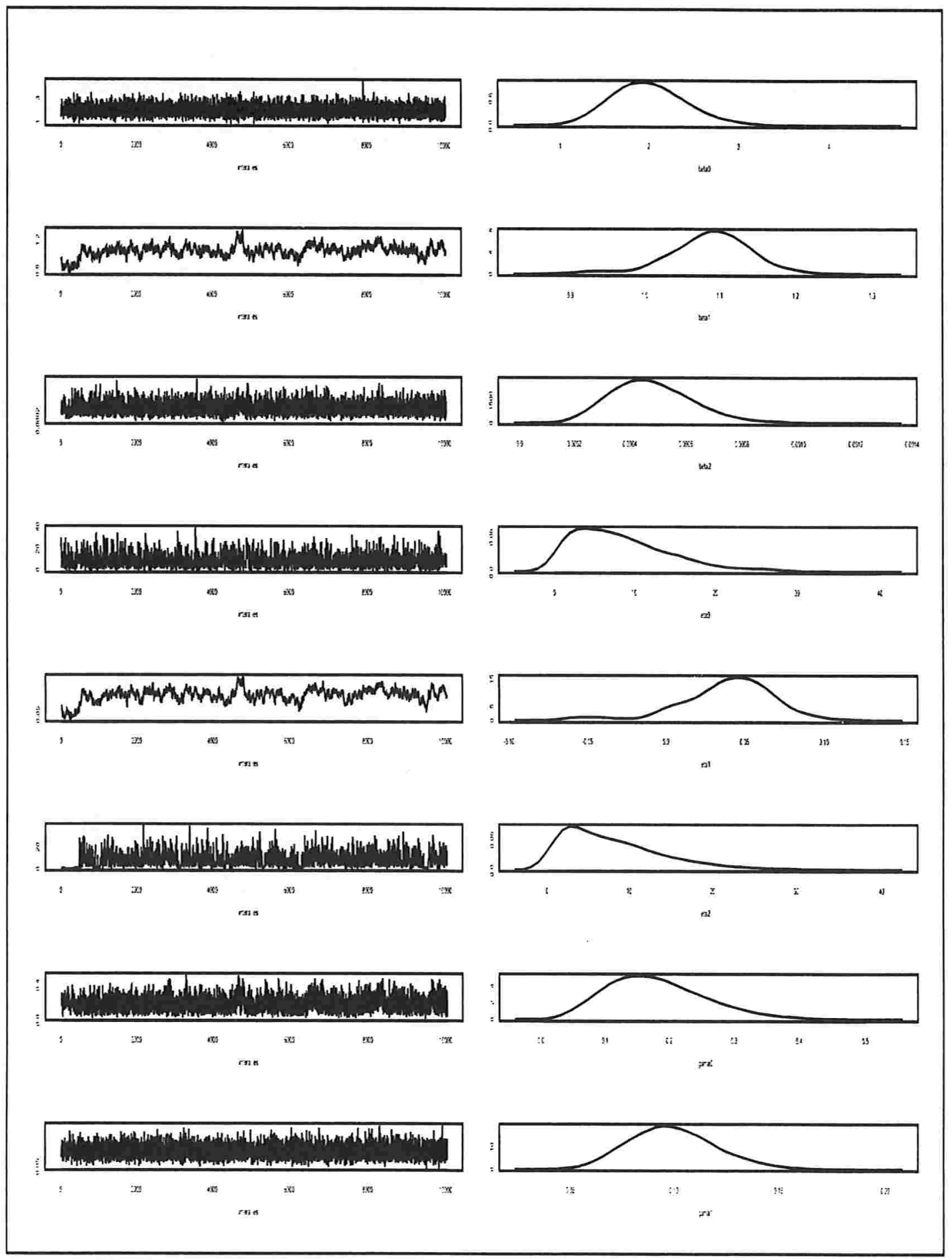

Figura 3.3: $\mathrm{O}$ traço e a Densidades a posteriori de $\beta_{0}, \beta_{1}, \xi_{1}, \xi_{2}$, $\gamma_{0}$ e $\gamma_{1}$.

Modelo M2 (superposição de processos dependentes sem a presença de covariáveis)

Assumimos uma superposição de dois PPNH polinomial, dependentes onde a covariável $x$ não afeta as intensidades e a correlação $\rho$. 
Considerando as distribuições a priori do modelo M2 com $J=3$ conhecidas, fixamos alguns valores para os hiperparâmetros (Vrs Hip), onde os resultados a posteriori estão sumarizados na Tabela 3.6, onde estão apresentadas características tais como média a posteriori, desvio-padrão (DP) e intervalos de credibilidade de $95 \%$ para os parâmetros de interesse. Também apresentamos o fator de escala de redução $\hat{R}$ (Gelman e Rubin, 1992) para os parâmetros $\beta_{0}, \beta_{1}, \beta_{2}, \rho$ do modelo M2. Com valores diferentes dos hiperparâmetros observamos que as médias a posteriori são próximas para cada valor real parâmetros do modelo, variando apenas os erros-padrão e os resultados para as cadeias geradas foram satisfatórios; isso pode ser visto graficamente na Figura 3.4 que apresenta o traço e a densidade dos parâmetros de interesse.

Tabela 3.6: Resumos a posteriori para o modelo M2.

\begin{tabular}{c|c|c|c|c|c}
\hline Parâmetro & Vrs. Hip & Média & D.P & Interv. de Credibilidade & $\hat{R}$ \\
\hline \hline \multirow{6}{*}{$\beta_{0}$} & $\Gamma(24 ; 12)$ & 1,978 & 0,3987 & $(1,275 ; 2,837)$ & 1,029 \\
\cline { 2 - 6 } & $\Gamma(48 ; 24)$ & 1,988 & 0,2858 & $(1,466 ; 2,586)$ & 1,015 \\
\cline { 2 - 6 } & $\Gamma(36 ; 18)$ & 1,986 & 0,3278 & $(1,395 ; 2,668)$ & 1,032 \\
\hline \multirow{3}{*}{$\beta_{1}$} & $\Gamma(0,01 ; 0,01)$ & 1,086 & 0,02509 & $(1,037 ; 1,135)$ & 1,035 \\
\cline { 2 - 6 } & $\Gamma(1 ; 0,01)$ & 1,089 & 0,03434 & $(1,022 ; 1,156)$ & 1,001 \\
\cline { 2 - 6 } & $\Gamma(10 ; 10)$ & 1,087 & 0,03991 & $(1,01 ; 1,166)$ & 1,016 \\
\hline \multirow{2}{*}{$\beta_{2}$} & $\Gamma(10 ; 20000)$ & 0,0004824 & 0,0001255 & $(0,00025559 ; 0,000745)$ & 1,012 \\
\cline { 2 - 6 } & $\Gamma(20 ; 40000)$ & 0,0004938 & 0,0001003 & $(0,0003138 ; 0,0007052)$ & 1,006 \\
\cline { 2 - 6 } & $\Gamma(100 ; 200000)$ & 0,0004989 & 0,00004876 & $(0,0004082 ; 0,0005987)$ & 1,002 \\
\hline \multirow{2}{*}{$\rho$} & $\Gamma(45 ; 30)$ & 0,6011 & 0,05435 & $(0,4925 ; 0,7046)$ & 1,013 \\
\cline { 2 - 6 } & $\Gamma(18 ; 12)$ & 0,6052 & 0,07764 & $(0,4491 ; 0,7504)$ & 1,005 \\
\cline { 2 - 6 } & $\Gamma(12,8)$ & 0,6008 & 0,08906 & $(0,4217 ; 0,7694)$ & 1,051 \\
\hline \hline
\end{tabular}

Modelo M3 (superposição de processos dependentes na presença de uma covariável)

Neste caso, vamos considerar a superposição de um PPNH polinomial, dependentes onde a covariável afeta as intensidades individuais para cada componente, mas não afeta o parâmetro de correlação $\rho$.

Considerando as distribuições a priori do modelo $\mathrm{M} 3 \mathrm{com} J=3$ conhecidas, na Tabela 3.7, apresentamos resumos a posteriori correspondentes aos parâmetros $\beta_{0}, 3_{1}, 3_{2}, \xi_{0}, \xi_{1}, \xi_{2}$ e $\rho$, onde fixamos diferentes valores para os hiperparâmetros. Nesta Tabela estão apresentadas características tais como média a posteriori, desvio-padrão (DP) e intervalos de credibilidade de $95 \%$ para os parâmetros de interesse. Também é mostrado o fator de 

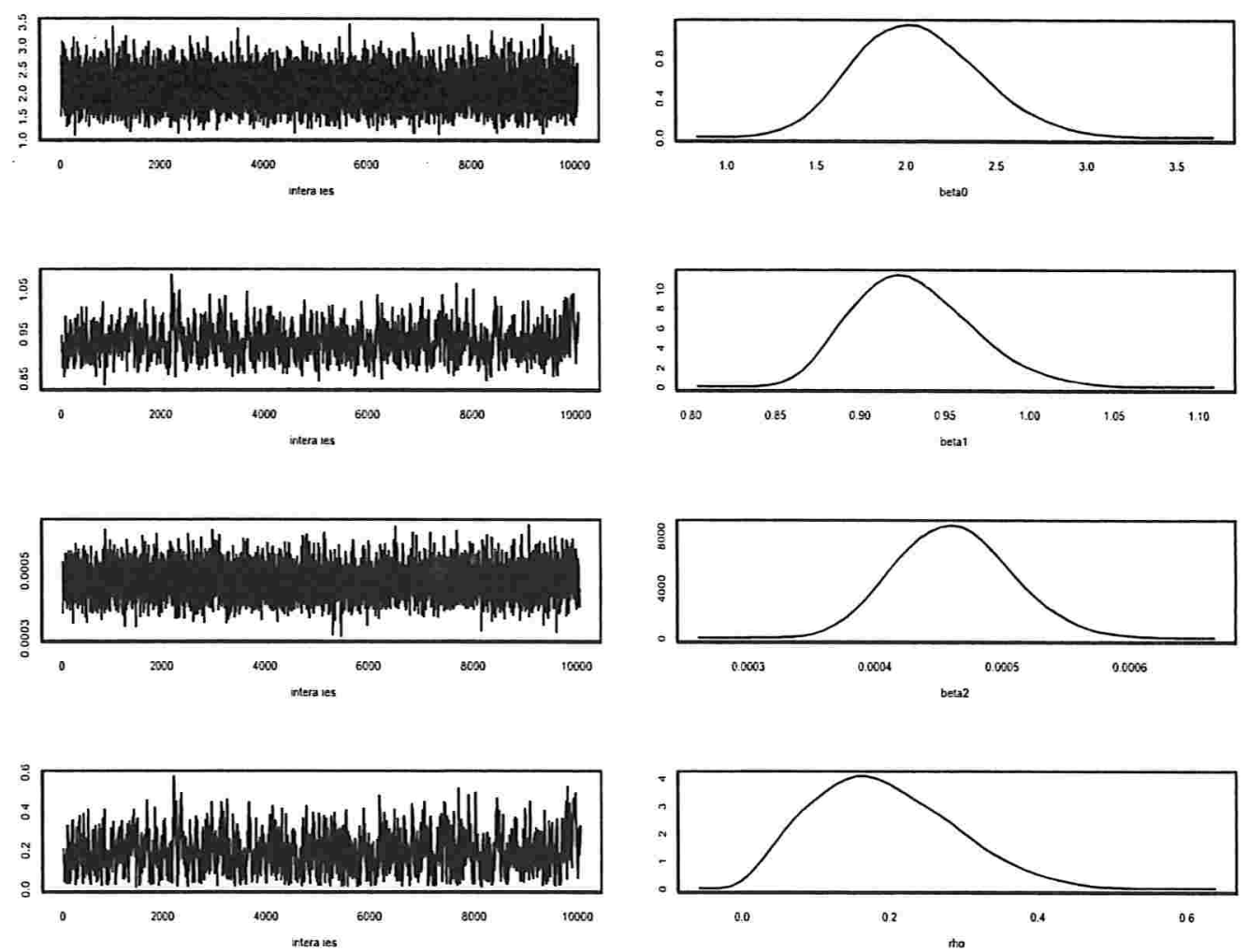

Figura 3.4: O traço e a Densidades a posteriori de $\beta_{0}, \beta_{1}, \beta_{2}$ e $\rho$.

escala de redução $\hat{R}$ para os parâmetros $\beta_{0}, \beta_{1}, \beta_{2}, \xi_{0}, \xi_{1}, \xi_{2}$ e $\rho$ do modelo M2. Com valores diferentes dos hiperparâmetros as médias a posteriori são próximas para cada parâmetros do modelo, variando apenas os erros-padrão e os resultados para as cadeias geradas foram satisfatórios, e isso pode ser visto graficamente na Figura 3.5 que apresenta o traço e a densidade dos parâmetros de interesse. Também temos $\hat{R}$ menores do que 1,1 para todos os casos, o que indica convergência do algoritmo.

Modelo M4 (superposição de processos dependentes na presença de uma covariável)

Neste caso, vamos considerar a superposição de PPNH polinomiais, dependentes onde a covariável afeta as intensidades individuais para cada componente e o parâmetro de correlação $\rho$.

Considerando as distribuições a priori do modelo $\mathrm{M} 4 \mathrm{com} J=3$ conhecidas, temos na Tabela 3.8 os sumários a posteriori de interesse correspondentes aos paràmetros $\beta_{0}, \beta_{1}, \beta_{2}$, 
Tabela 3.7: Resumos a posteriori para o modelo M3.

\begin{tabular}{c|c|c|c|c|c}
\hline Parâmetro & Vrs. Hip & Média & D.P & Interv. de Credibilidade & $\hat{R}$ \\
\hline \hline \multirow{6}{*}{$\beta_{0}$} & $\Gamma(24 ; 12)$ & 1,994 & 0,4058 & $(1,283 ; 2,864)$ & 1,0105 \\
\cline { 2 - 6 } & $\Gamma(12 ; 6)$ & 1,992 & 0,5776 & $(1,021 ; 3,272)$ & 1,019 \\
\cline { 2 - 6 } & $\Gamma(400 ; 200)$ & 2,00 & 0,09979 & $(1,809 ; 2,202)$ & 1,007 \\
\hline \multirow{3}{*}{$\beta_{1}$} & $\Gamma(0,01 ; 0,01)$ & 1,132 & 0,05775 & $(1,026 ; 1,254)$ & 1,031 \\
\cline { 2 - 6 } & $\Gamma(20 ; 10)$ & 1,078 & 0,0748 & $(0,9354 ; 1,236)$ & 1,093 \\
\cline { 2 - 6 } & $\Gamma(1 ; 0,01)$ & 1,085 & 0,0446 & $(1,006 ; 1,182)$ & 1,001 \\
\hline \multirow{3}{*}{$\beta_{2}$} & $\Gamma(10 ; 20000)$ & 0,000481 & 0,0001531 & $(0,0002292 ; 0,000823)$ & 1,001 \\
\cline { 2 - 6 } & $\Gamma(50 ; 100000)$ & 0,0004969 & 0,000006978 & $(0,0003694 ; 0,0006434)$ & 1,009 \\
\cline { 2 - 6 } & $\Gamma(100 ; 200000)$ & 0,0005448 & 0,0000523 & $(0,0004473 ; 0,000652)$ & 1,008 \\
\hline \multirow{5}{*}{$\xi_{0}$} & $N(0 ; 100)$ & 7,944 & 6,017 & $(-0,05079 ; 22,25)$ & 1,003 \\
\cline { 2 - 6 } & $\Gamma(5 ; 1)$ & 5,511 & 2,81 & $(0,5811 ; 11,35)$ & 1,056 \\
\cline { 2 - 6 }$\xi_{1}$ & $\Gamma(5 ; 10)$ & 9,996 & 0,9984 & $(8,039 ; 11,95)$ & 1,001 \\
\hline \multirow{2}{*}{$\xi_{2}$} & $N(0 ; 100)$ & 0,0277 & 0,03376 & $(-0,004679 ; 0,08451)$ & 1,067 \\
\cline { 2 - 6 } & $\Gamma(0 ; 0,1)$ & 0,04807 & 0,0205 & $(0,007921 ; 0,08769)$ & 1,006 \\
\cline { 2 - 6 } & $\Gamma(0 ; 1)$ & 0,04038 & 0,02408 & $(-0,004939 ; 0,09017)$ & 1,002 \\
\cline { 2 - 6 } & $\Gamma(0 ; 100)$ & 6,891 & 6,017 & $(0,2756 ; 21,52)$ & 1,028 \\
\cline { 2 - 6 } & $\Gamma(5 ; 1)$ & 4,948 & 1,022 & $(2,937 ; 6,89)$ & 1,052 \\
\hline \multirow{2}{*}{$\rho$} & $\Gamma(6 ; 1)$ & 5,985 & 1,004 & $(4,0 ; 7,953)$ & 1,009 \\
\cline { 2 - 6 } & $\Gamma(24 ; 16)$ & 0,5702 & 0,05365 & $(0.464 ; 0,6763)$ & 1,006 \\
\cline { 2 - 6 } & $\Gamma(18 ; 12)$ & 0,5663 & 0,09139 & $(0,3837 ; 0,7378)$ & 1,014 \\
\cline { 2 - 6 } & $\Gamma(6,4)$ & 0,6182 & 0,1617 & $(0,2737 ; 0,8906)$ & 1,048 \\
\hline \hline
\end{tabular}

$\xi_{0}, \xi_{1}, \xi_{2}, \gamma_{0}$ e $\gamma_{1}$, onde fixamos diferentes valores para os hiperparâmetros. Nesta Tabela estão apresentadas características tais como média a posteriori, desvio-padrão (DP) e intervalos de credibilidade de $95 \%$ para os parâmetros de interesse. Também é mostrado o fator de escala de redução $\hat{R}$ para os parâmetros $\beta_{0} \beta_{1}, \beta_{2}, \xi_{0}, \xi_{1}, \gamma_{0}$ e $\gamma_{1}$ do modelo M4. Com valores diferentes dos hiperparâmetros as médias a posteriori são próximas para cada parâmetros do modelo, variando apenas os erros-padrão e os resultados para as cadeias geradas foram satisfatórios, e isso pode ser visto graficamente na Figura 5.2 que apresenta o traço e a densidade dos parâmetros de interesse. Também temos $\hat{R}$ menores do que 1,1 para todos os casos, o que indica convergência do algoritmo.

\subsubsection{Exemplo 3}

Na Tabela 3.9, apresentamos um conjunto de dados simulados a partir da superposição de PPNH polinomiais com função intensidade clada por

$$
\lambda_{1}\left(t_{i}\right)=3_{0} e^{-\xi_{0} x_{i}}+\beta_{1} t_{i} e^{-\xi_{1} x_{i}}+3_{2} t_{i}^{2} e^{-\xi_{2} x_{i}}+3_{3} t_{i}^{3} e^{-\xi_{3} x_{i}},
$$




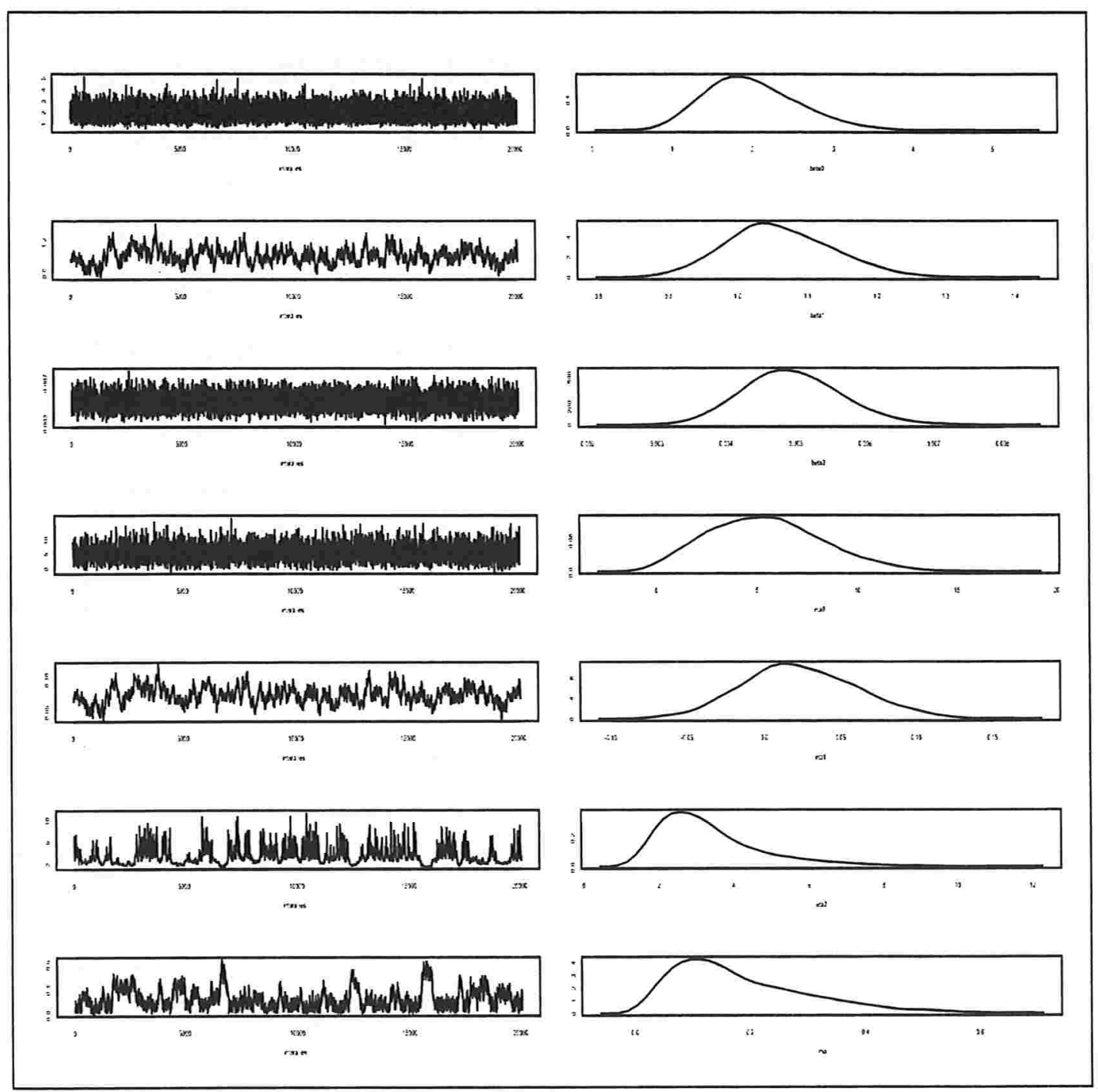

Figura 3.5: O traço e a Densidades a posteriori de $\beta_{0}, \beta_{1}, \beta_{2}, \xi_{0}, \xi_{1}, \xi_{2}$ e $\rho$.

para os valores fixados $\beta_{0}=2, \beta_{1}=0,91, \beta_{2}=2 \times 10^{-4}, \beta_{3}=1,0 \times 10^{-6}, \xi_{0}=15, \xi_{1}=$ $0,001, \xi_{2}=20$ e $\xi_{3}=25 ; 50$ observações foram geradas considerando uma covariável com valores 1 e 2 . Observar que na geração desse dados assumimos $\rho=0,70$.

Os resultados da análise dos modelos foram obtido atráves de métodos MCMC implementado no software WINBUGS (Spiegelhalter et al., 1997) após descartar as 4000 primeiras observações (burn-in).

A seguir apresentamos os sumários a posterior considerado os modelos propostos previamente. 
Tabela 3.8: Resumos a posteriori para o modelo M4.

\begin{tabular}{c|c|c|c|c|c}
\hline Parâmetro & Vrs. Hip & Média & D.P & Interv. de Credibilidade & $\hat{R}$ \\
\hline \hline \multirow{6}{*}{$\beta_{0}$} & $\Gamma(24 ; 12)$ & 1,995 & 0,4079 & $(1,277 ; 2,87)$ & 1,002 \\
\cline { 2 - 6 } & $\Gamma(48 ; 28)$ & 1,995 & 0,2884 & $(1,471 ; 2,602)$ & 1,023 \\
\cline { 2 - 6 } & $\Gamma(72 ; 36)$ & 1,998 & 0,2347 & $(1,561 ; 2,482)$ & 1,003 \\
\hline \multirow{5}{*}{$\beta_{1}$} & $\Gamma(0,01 ; 0,01)$ & 1,068 & 0,0558 & $(0,9477 ; 1,169)$ & 1,062 \\
\cline { 2 - 6 } & $\Gamma(60 ; 60)$ & 1,068 & 0,06659 & $(0,9435 ; 1,184)$ & 1,024 \\
\cline { 2 - 6 } & $\Gamma(1 ; 0,01)$ & 1,085 & 0.0446 & $(1,006 ; 1,182)$ & 1,001 \\
\hline \multirow{5}{*}{$\beta_{2}$} & $\Gamma(10 ; 20000)$ & 0,0004697 & 0,0001502 & $(0,000223 ; 0,0008061)$ & 1,003 \\
\cline { 2 - 6 } & $\Gamma(100 ; 200000)$ & 0,0004908 & 0,00004904 & $(0,0003992 ; 0,0005914)$ & 1,011 \\
\cline { 2 - 6 }$\xi_{0}$ & $\Gamma(20 ; 40000)$ & 0,0004734 & 0,0001082 & $(0,0002865 ; 0,0007086)$ & 1,014 \\
\cline { 2 - 6 } & $N(0 ; 100)$ & 9,032 & 6,053 & $(0,8472 ; 23,33)$ & 1,032 \\
\cline { 2 - 6 } & $\Gamma(8 ; 1)$ & 8,014 & 0,9993 & $(6,067 ; 9,981)$ & 1,052 \\
\cline { 2 - 6 }$\xi_{1}$ & $\Gamma(10 ; 1)$ & 9,996 & 0,9984 & $(8,039 ; 11,95)$ & 1,001 \\
\cline { 2 - 6 } & $N(0 ; 0,01)$ & 0,0277 & 0,03376 & $(-0,004679 ; 0,08451)$ & 1,067 \\
\cline { 2 - 6 } & $\Gamma(0 ; 0,1)$ & 0,01512 & 0,04742 & $(-0,07501 ; 0,09035)$ & 1,008 \\
\cline { 2 - 6 }$\xi_{2}$ & $\Gamma(0 ; 1)$ & 0,04038 & 0,02408 & $(-0,004939 ; 0,09017)$ & 1,002 \\
\hline & $\Gamma(0 ; 0,01)$ & 6,891 & 6,017 & $(0,2756 ; 21,52)$ & 1,028 \\
\cline { 2 - 6 } & $\Gamma(5 ; 1)$ & 4,948 & 1,022 & $(2,937 ; 6,89)$ & 1,052 \\
\cline { 2 - 6 } & $\Gamma(6 ; 1)$ & 5,985 & 1,004 & $(4,0 ; 7,953)$ & 1,009 \\
\hline \multirow{5}{*}{$\gamma_{0}$} & $\Gamma(18 ; 54)$ & 0,2376 & 0,04916 & $(0,1496 ; 0,3411)$ & 1,002 \\
\cline { 2 - 6 } & $\Gamma(12 ; 36)$ & 0,2302 & 0,0589 & $(0,1269 ; 0,3558)$ & 1,003 \\
\cline { 2 - 6 } & $\Gamma(9,27)$ & 0,2354 & 0,06525 & $(0,1199 ; 0,373)$ & 1,009 \\
\hline \multirow{5}{*}{$\gamma_{1}$} & $\Gamma(33 ; 187)$ & 0,1484 & 0,02372 & $(0,1049 ; 0,1978)$ & 1,028 \\
\cline { 2 - 6 } & $\Gamma(30 ; 170)$ & 0,1465 & 0,02462 & $(0,1015 ; 0,1978)$ & 1,001 \\
\cline { 2 - 6 } & $\Gamma(24,136)$ & 0,1455 & 0,02755 & $(0,09593 ; 0,2036)$ & 1,004 \\
\hline \hline
\end{tabular}

\section{Modelo M2 (superposição de processos sem a presença de covariáveis)}

Assumimos uma superposição de dois PPNHs polinomiais, dependentes onde a covariável $x$ não afeta as intensidades nem correlação $\rho$.

Considerando as distribuições a priori dadas para o modelo M2 com $J=4$ conhecidas, fixamos alguns valores para os hiperparâmetros (Vrs Hip), onde os resultados a posteriori estão sumarizados na Tabela 3.10. Observamos que com valores diferentes dos hiperparâmetros as médias a posteriori são próximas para cada parâmetros do modelo, variando apenas os erros-padrão e os resultados para as cadeias geradas foram satisfatórios, e isso pode ser visto graficamente na Figura 3.9 que apresenta o traço e a clensidade dos parâmetros de interesse. 


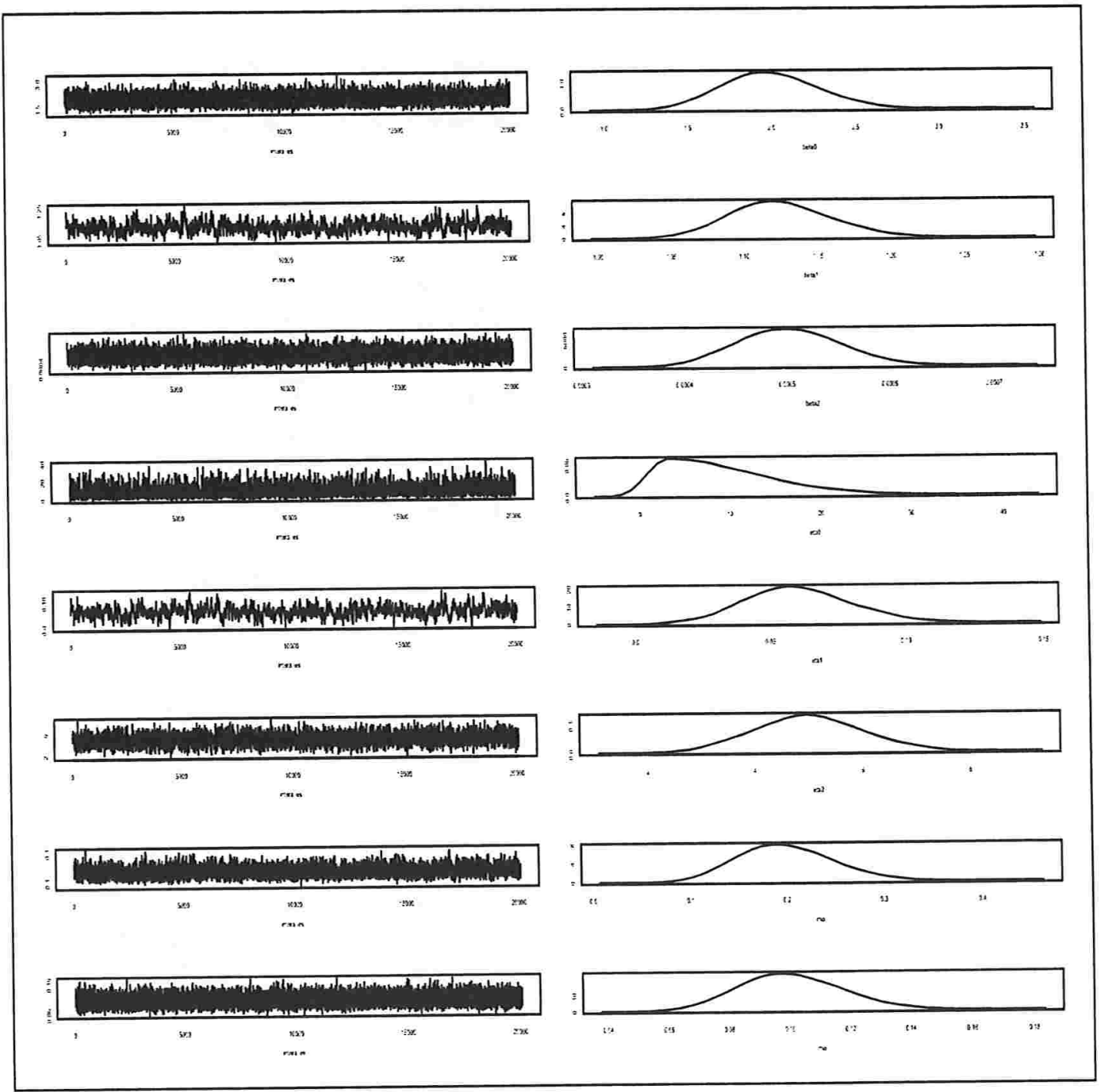

Figura 3.6: $\mathrm{O}$ traço e a Densidades a posteriori de $\beta_{0}, \beta_{1}, \beta_{2}, \xi_{0}, \xi_{1}, \xi_{2}$, $\gamma_{0}$ e $\gamma_{1}$.

Modelo M3 ( superposição de processos dependentes na presença de uma covariável )

Neste caso, vamos consiclerar a superposição de PPNHs polinomiais, dependentes onde a covariável afeta as intensidades individuais para cada componente, mas não afeta o parâmetro de correlação $\rho$.

Considerando as distribuições a priori do modelo M3 com $J=4$ conhecidas, temos na Tabela 3.11, os resumos a posteriori correspondentes aos paràmetros $\beta_{0}, \beta_{1}, \beta_{2}$, $\xi_{3}, \xi_{0}, \xi_{1}, \xi_{2}, \xi_{3}$ e $\rho$. Observamos que com valores diferentes dos hiperparàmetros as médias a posteriori são próximas para cada paràmetros do modelo, variando apenas os erros-padrão e os resultados para as cadleias geradas foram satisfatórios, e isso pode 
Tabela 3.9: Dados gerados a partir de um processo PPNH.

\begin{tabular}{c|c|c|c|c|c|c|c|c|c}
\hline \hline$t_{i}$ & $x_{i}$ & $t_{i}$ & $x_{i}$ & $t_{i}$ & $x_{i}$ & $t_{i}$ & $x_{i}$ & $t_{i}$ & $x_{i}$ \\
\hline 9,0226 & 1 & 163,9241 & 2 & 234,1622 & 1 & 320,3625 & 2 & 372,4804 & 1 \\
13,6092 & 1 & 172,6444 & 2 & 237,2154 & 1 & 333,9281 & 1 & 374,1010 & 1 \\
25,0062 & 2 & 173,3407 & 2 & 256,3211 & 1 & 335,3652 & 2 & 379,0545 & 1 \\
28,8325 & 1 & 190,2042 & 1 & 257,8515 & 2 & 341,1776 & 2 & 381,3608 & 1 \\
41,0224 & 2 & 191,4878 & 2 & 266,2266 & 2 & 345,9216 & 2 & 381,6248 & 2 \\
60,2645 & 1 & 193,1681 & 1 & 269,6066 & 1 & 346,7785 & 1 & 386,6440 & 1 \\
69,4851 & 1 & 195,3098 & 1 & 275,1535 & 1 & 352,9118 & 2 & 387,2999 & 1 \\
129,9379 & 2 & 209,3594 & 1 & 299,9811 & 1 & 354,5584 & 1 & 391,2027 & 1 \\
130,5394 & 2 & 216,4074 & 1 & 308,4226 & 1 & 356,2139 & 1 & 392,5856 & 1 \\
156,2600 & 2 & 229,3982 & 1 & 313,0338 & 2 & 372,1163 & 1 & 397,0635 & 1 \\
\hline \hline
\end{tabular}

Tabela 3.10: Resumos a posteriori para o modelo M2.

\begin{tabular}{c|c|c|c|c|c}
\hline Parâmetro & Vrs. Hip & Média & D.P & Interv. de Credibilidade & $\hat{R}$ \\
\hline \hline \multirow{2}{*}{$\beta_{0}$} & $\Gamma(24 ; 12)$ & 2,133 & 0,425 & $(1,38 ; 3,037)$ & 1,004 \\
\cline { 2 - 6 } & $\Gamma(48 ; 24)$ & 2,014 & 0,29 & $(1,485 ; 2,625)$ & 1,007 \\
\hline \multirow{2}{*}{$\beta_{1}$} & $\Gamma(0,001 ; 0,0001)$ & 0,952 & 0,02239 & $(0,9029 ; 0,99)$ & 1,049 \\
\cline { 2 - 6 } & $\Gamma(0,01 ; 0,01)$ & 0,9515 & 0,02243 & $(0,9023 ; 0,9897)$ & 1,010 \\
\hline \multirow{2}{*}{$\beta_{2}$} & $\Gamma(20 ; 2000)$ & 0,0002068 & 0,00004347 & $(0,0001292 ; 0,000299)$ & 1,006 \\
\cline { 2 - 6 } & $\Gamma(10 ; 10000)$ & 0,0001273 & 0,00003839 & $(0,000006257 ; 0,0002124)$ & 1,008 \\
\hline \multirow{2}{*}{$\beta_{3}$} & $\Gamma(50 ; 200000)$ & $1,315 E-6$ & $1,021 E-9$ & $(1,051 E-6 ; 1,596 E-6)$ & 1,014 \\
\cline { 2 - 6 } & $\Gamma(100 ; 2000)$ & $1,86 E-6$ & $1,39 E-7$ & $(1,596 E-6 ; 2,139 E-6)$ & 1,013 \\
\hline$\rho$ & $\Gamma(48 ; 12)$ & 0,7998 & 0,05124 & $(0,6907 ; 0,8901)$ & 1,001 \\
\cline { 2 - 6 } & $\Gamma(40 ; 10)$ & 0,7998 & 0,05607 & $(0,6793 ; 0,8038)$ & 1,007 \\
\hline \hline
\end{tabular}

ser visto graficamente na Figura 3.10 que apresenta o traço e a densidade dos parâmetros de interesse. Também temos $\hat{R}$ menores do que 1,1 para todos os casos, o que indica convergência do algoritmo.

Modelo M4 (superposição de processos dependentes na presença de uma covariável)

Neste caso, vamos considerar a superposição de PPNHs polinomiais, dependentes onde a covariável afeta as intensidades indivicluais para cacla componente e o parâmetro cle correlação $\rho$.

Considerando as distribuições a priori do modelo M4 conhecidas, na Tabela 3.12, apresentamos resumos a posteriori correspondentes aos paràmetros $\beta_{0}, \beta_{1}, \beta_{2}, \beta_{3}, \xi_{0}, \xi_{1}, \xi_{2}, \xi_{3}, \gamma_{0}$ e $\gamma_{1}$, onde fixamos diferentes valores para os hiperparâmetros. Nesta Tabela estão apresentadas características tais como média a posteriori, desvio-padrão (DP) e intervalos de credibilidade de $95 \%$ para os parâmetros de interesse. Também é mostrado o fator de 


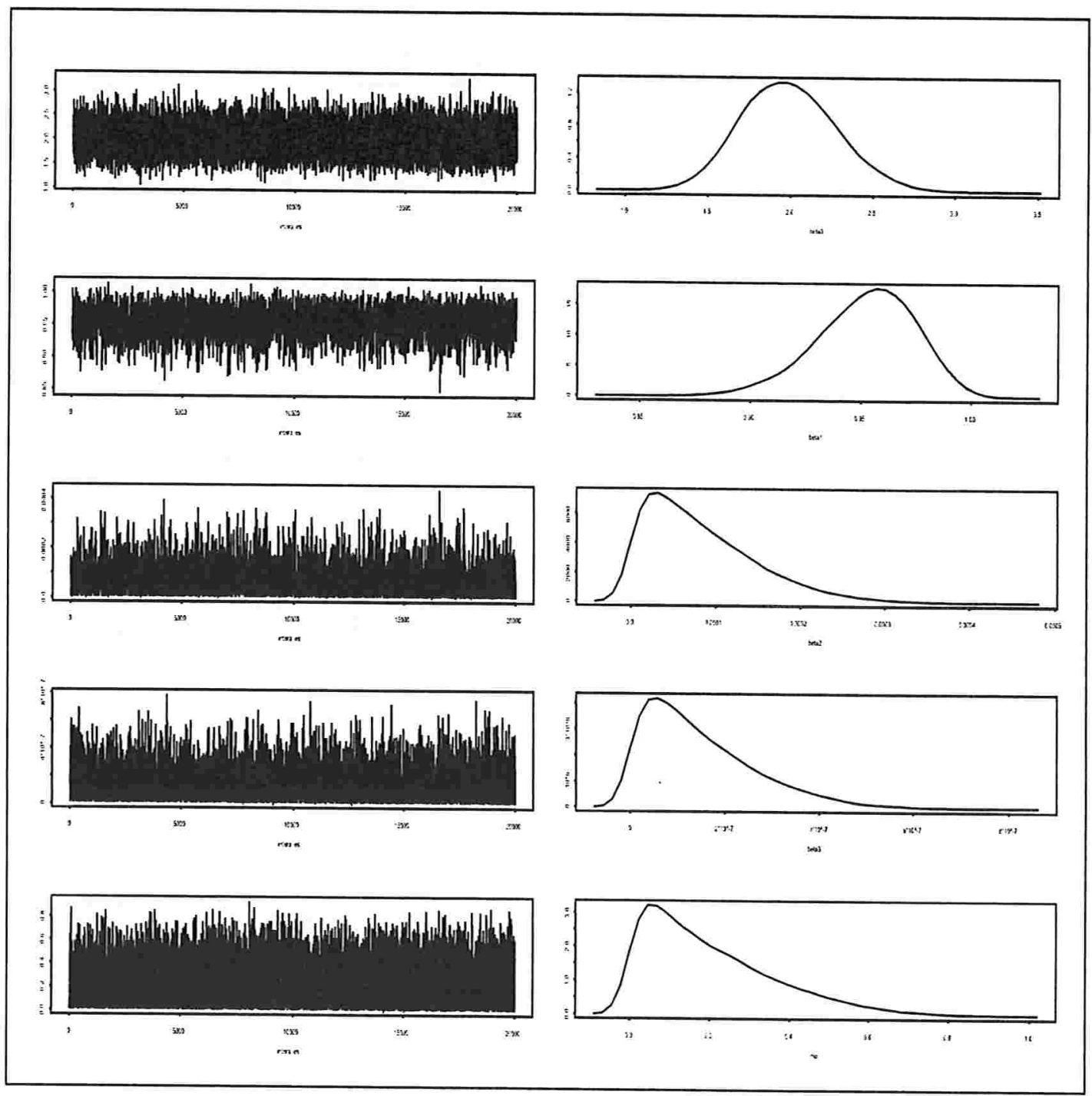

Figura 3.7: O traço e a Densidades a posteriori de $\beta_{0}, \beta_{1}, \beta_{2}, \beta_{3}$ e $\rho$.

escala de redução $\hat{R}$ para os parâmetros $\beta_{0}, \beta_{1}, \beta_{2}, \beta_{3}, \xi_{0}, \xi_{1}, \xi_{2}, \xi_{3}, \gamma_{0}$ e $\gamma_{1}$ do modelo M4. Com valores diferentes dos hiperparâmetros as médias a posteriori são próximas para cada parâmetros do modelo, variando apenas os erros-padrão e os resultados para as cadeias geradas foram satisfatórios, e isso pode ser visto graficamente na Figura 3.11 que apresenta o traço e a densidade dos paràmetros de interesse. Também temos $\hat{R}$ menores do que 1,1 para todos os casos, o que indica convergência do algoritmo.

\subsection{Seleção de modelos}

Considerando os processos de seleção de modelos vistos na seção 1.3.2, utilizamos o método DIC implementado no pacote estatístico WinBugs (ver Spiegelhalter et al., 2002) 
Tabela 3.11: Resumos a posteriori para o modelo M3.

\begin{tabular}{c|c|c|c|c|c}
\hline Parâmetro & Vrs. Hip & Média & D.P & Interv. de Credibilidade & $\hat{R}$ \\
\hline \hline \multirow{2}{*}{$\beta_{0}$} & $\Gamma(48 ; 24)$ & 2,086 & 0,2976 & $(1,543 ; 2,707)$ & 1,002 \\
\cline { 2 - 6 } & $\Gamma(12 ; 6)$ & 1,931 & 0,5388 & $(1,011 ; 3,124)$ & 1,003 \\
\hline \multirow{2}{*}{$\beta_{1}$} & $\Gamma(120 ; 60)$ & 0,9017 & 0,01469 & $(0,872 ; 0,9296)$ & 1,001 \\
\cline { 2 - 6 } & $\Gamma(40 ; 20)$ & 0,951 & 0,01955 & $(0,9094 ; 0,9857)$ & 1,002 \\
\hline \multirow{2}{*}{$\beta_{2}$} & $\Gamma(50 ; 250000)$ & 0,0001494 & 0,00002086 & $(0,0001113 ; 0,0001931)$ & 1,003 \\
\cline { 2 - 6 } & $\Gamma(3 ; 100000)$ & 0,0001081 & $0,000050.42$ & $(0,0001113 ; 0,0001931)$ & 1,003 \\
\hline \multirow{2}{*}{$\beta_{3}$} & $\Gamma(50 ; 50000)$ & $1,83 E-6$ & $1,273 E-7$ & $(1,118 E-6 ; 1,44 E-6)$ & 1,005 \\
\cline { 2 - 6 } & $\Gamma(1 ; 1000000)$ & 0,0000001004 & 0,00000008763 & $(1,18 E-6 ; 31,931 E-6)$ & 1,005 \\
\hline \multirow{2}{*}{$\xi_{0}$} & $N(15 ; 100)$ & 14,98 & 10,06 & $(-4,668 ; 34.61)$ & 1,004 \\
\cline { 2 - 6 } & $N(5 ; 100)$ & 4.987 & 10,00 & $(-14,67 ; 24,5)$ & 1,001 \\
\hline \multirow{3}{*}{$\xi_{1}$} & $N(0 ; 100)$ & 0,02526 & 9,973 & $(-19,58 ; 19,45)$ & 1,007 \\
\cline { 2 - 6 } & $\Gamma(0 ; 100)$ & 0,002363 & 9,988 & $(-19.55 ; 19,59)$ & 1,003 \\
\hline \multirow{2}{*}{$\xi_{2}$} & $N(18 ; 100)$ & 18,0 & 10,04 & $(-1,678 ; 37,69)$ & 1,002 \\
\cline { 2 - 6 } & $N(20 ; 100)$ & 19,98 & 9,991 & $(0,2105 ; 39,52)$ & 1,008 \\
\hline \multirow{2}{*}{$\xi_{3}$} & $\Gamma(25 ; 10)$ & 24,99 & 3,175 & $(18,75 ; 31,21)$ & 1,002 \\
\cline { 2 - 6 } & $\Gamma(25 ; 100)$ & 24,96 & 10,01 & $(5,369 ; 44,65)$ & 1,004 \\
\hline \multirow{2}{*}{$\rho$} & $\Gamma(48 ; 12)$ & 0,7999 & 0,05116 & $(0,6908 ; 0,8901)$ & 1,004 \\
\cline { 2 - 6 } & $\Gamma(16 ; 4)$ & 0,7999 & 0,08739 & $(0,6036 ; 0,9393)$ & 1,013 \\
\hline \hline
\end{tabular}

onde observamos dos resultados apresentados na Tabela 3.13 que para o modelo PPNH polinomial de primeiro grau o modelo M4 tem o o melhor ajuste; para o modelo PPNH polinomial de segundo grau o modelo M3 apresenta o melhor ajuste e para o modelo PPNH polinomial de terceiro grau o modelo M4 apresenta o melhor ajuste. 


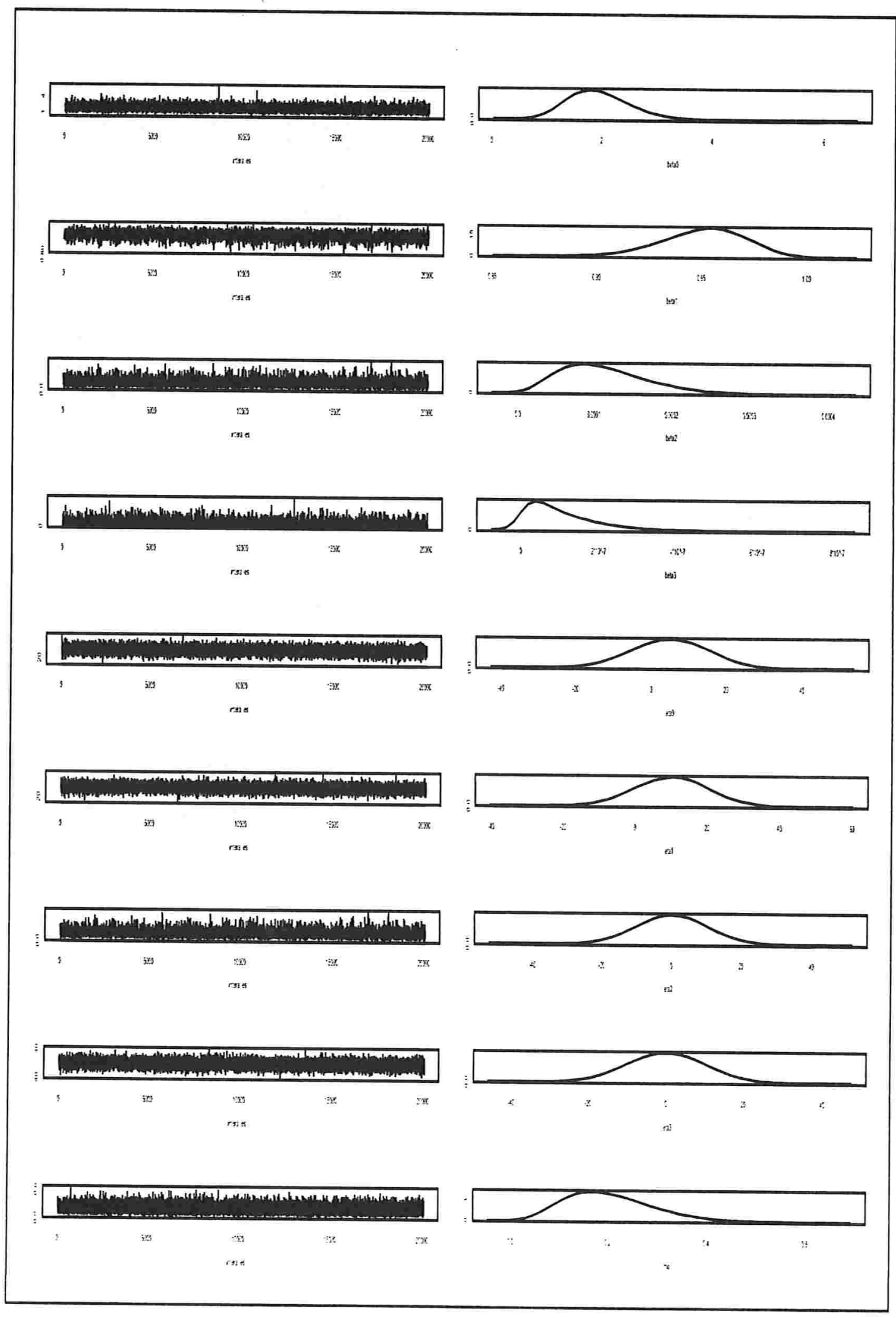

Figura 3.8: O traço e a Densidades a posteriori de $\beta_{0}, \beta_{1}, 3_{2}, \beta_{3}, \xi_{0}, \xi_{1}, \xi_{2}, \xi_{3}$ e $\rho$. 
Tabela 3.12: Resumos a posteriori para o modelo M4.

\begin{tabular}{|c|c|c|c|c|c|}
\hline Parâmetro & Vrs. Hip & Média & D.P & Interv. de Credibilidade & $\hat{R}$ \\
\hline \multirow[t]{3}{*}{$\beta_{0}$} & $\Gamma(24 ; 12)$ & 1,995 & 0,4079 & $(1,277 ; 2,87)$ & 1,002 \\
\hline & $\Gamma(48 ; 28)$ & 2,00 & 0,2919 & $(1,473 ; 2,609)$ & 1,011 \\
\hline & $\Gamma(72 ; 36)$ & 2,00 & 0,2358 & $(1,563 ; 2,486)$ & 1,005 \\
\hline \multirow[t]{3}{*}{$\beta_{1}$} & $\Gamma(0,01 ; 0,01)$ & 1,068 & 0,0558 & $(0,9477 ; 1,169)$ & 1,062 \\
\hline & $\Gamma(1 ; 1)$ & 1,103 & 0,03562 & $(1,036 ; 1,174)$ & 1,036 \\
\hline & $\Gamma(1 ; 0,01)$ & 1,085 & 0.0446 & $(1,006 ; 1,182)$ & 1,001 \\
\hline \multirow[t]{3}{*}{$\beta_{2}$} & $\Gamma(10 ; 20000)$ & 0,0004697 & 0,0001502 & $(0,000223 ; 0,0008061)$ & 1,003 \\
\hline & $\Gamma(100 ; 200000)$ & 0,0004966 & 0,0000496 & $(0,000404 ; 0,000599)$ & 1,009 \\
\hline & $\Gamma(20 ; 40000)$ & 0,0004838 & 0,0001083 & $(0,0002953 ; 0,0007177)$ & 1,008 \\
\hline \multirow[t]{3}{*}{$\beta_{3}$} & $\Gamma(10 ; 20000)$ & 0,0004697 & 0,0001502 & $(0,000223 ; 0,0008061)$ & 1,003 \\
\hline & $\Gamma(100 ; 200000)$ & 0,0004966 & 0,0000496 & $(0,000404 ; 0,000599)$ & 1,009 \\
\hline & $\Gamma(20 ; 40000)$ & 0,0004838 & 0,0001083 & $(0,0002953 ; 0,0007177)$ & 1,008 \\
\hline \multirow[t]{3}{*}{$\xi_{0}$} & $N(0 ; 0,01)$ & 8,047 & 6,025 & $(0,04972 ; 22,32)$ & 1,010 \\
\hline & $\Gamma(8 ; 1)$ & 8,014 & 0,9993 & $(6,067 ; 9,981)$ & 1,052 \\
\hline & $\Gamma(10 ; 1)$ & 9,996 & 0,9984 & $(8,039 ; 11,95)$ & 1,001 \\
\hline \multirow[t]{3}{*}{$\xi_{1}$} & $N(0 ; 0,01)$ & 0,0277 & 0,03376 & $(-0,004679 ; 0,08451)$ & 1,067 \\
\hline & $\Gamma(0 ; 0,1)$ & 0,04807 & 0,0205 & $(0,007921 ; 0,08769)$ & 1,006 \\
\hline & $\Gamma(0 ; 1)$ & 0,04038 & 0,02408 & $(-0,004939 ; 0,09017)$ & 1,002 \\
\hline \multirow[t]{3}{*}{$\xi_{2}$} & $\Gamma(0 ; 0,01)$ & 6,891 & 6,017 & $(0,2756 ; 21,52)$ & 1,028 \\
\hline & $\Gamma(5 ; 1)$ & 4,948 & 1,022 & $(2,937 ; 6,89)$ & 1,052 \\
\hline & $\Gamma(6 ; 1)$ & 5,985 & 1,004 & $(4,0 ; 7,953)$ & 1,009 \\
\hline \multirow[t]{3}{*}{$\xi_{3}$} & $\Gamma(0 ; 0,01)$ & 6,891 & 6,017 & $(0,2756 ; 21,52)$ & 1,028 \\
\hline & $\Gamma(5 ; 1)$ & 4,948 & 1,022 & $(2,937 ; 6,89)$ & 1,052 \\
\hline & $\Gamma(6 ; 1)$ & 5,985 & 1,004 & $(4,0 ; 7,953)$ & 1,009 \\
\hline \multirow[t]{3}{*}{$\gamma_{0}$} & $\Gamma(4 ; 16)$ & 0,1698 & 0,07508 & $(0.05174 ; 0,3407)$ & 1,002 \\
\hline & $\Gamma(12 ; 48)$ & 0,1839 & 0,04651 & $(0,102 ; 0,2832)$ & 1,007 \\
\hline & $\Gamma(2,8)$ & 0,1371 & 0,08384 & $(0,01925 ; 0,3377)$ & 1,002 \\
\hline \multirow[t]{3}{*}{$\gamma_{1}$} & $\Gamma(20 ; 180)$ & 0,090816 & 0,02078 & $(0,06132 ; 0,1425)$ & 1,001 \\
\hline & $\Gamma(30 ; 270)$ & 0,09828 & 0,0167 & $(0,06769 ; 0,1332)$ & 1,006 \\
\hline & $\Gamma(10,90)$ & 0,09572 & 0,02854 & $(0,04751 ; 0,1582)$ & 1,003 \\
\hline
\end{tabular}

Tabela 3.13: Resultados obtidos para o Critério DIC.

\begin{tabular}{|c|c|c|c|c|}
\hline & Modelo & $\mathrm{N}^{\circ}$ Obs. & DIC & N. ${ }^{\circ}$ Parâmetros \\
\hline \multirow{3}{*}{$\mathrm{J}=2$} & $\backslash 2$ & 50 & 344,813 & 3 \\
\hline & $\mathrm{M}[3$ & 50 & 345,206 & 5 \\
\hline & MI4 & 50 & 344.313 & 6 \\
\hline \multirow{3}{*}{$\mathrm{J}=3$} & $\mathrm{M} 2$ & 50 & 357.657 & 4 \\
\hline & MI3 & 50 & 357.136 & $\overline{7}$ \\
\hline & M4 & 50 & 362,622 & 10 \\
\hline \multirow{3}{*}{$\mathrm{J}=3$} & $\mathrm{MI} 2$ & 50 & +22.880 & 5 \\
\hline & \2 & 50 & 122.83 & 9 \\
\hline & .14 & 50 & 364,166 & 11 \\
\hline
\end{tabular}




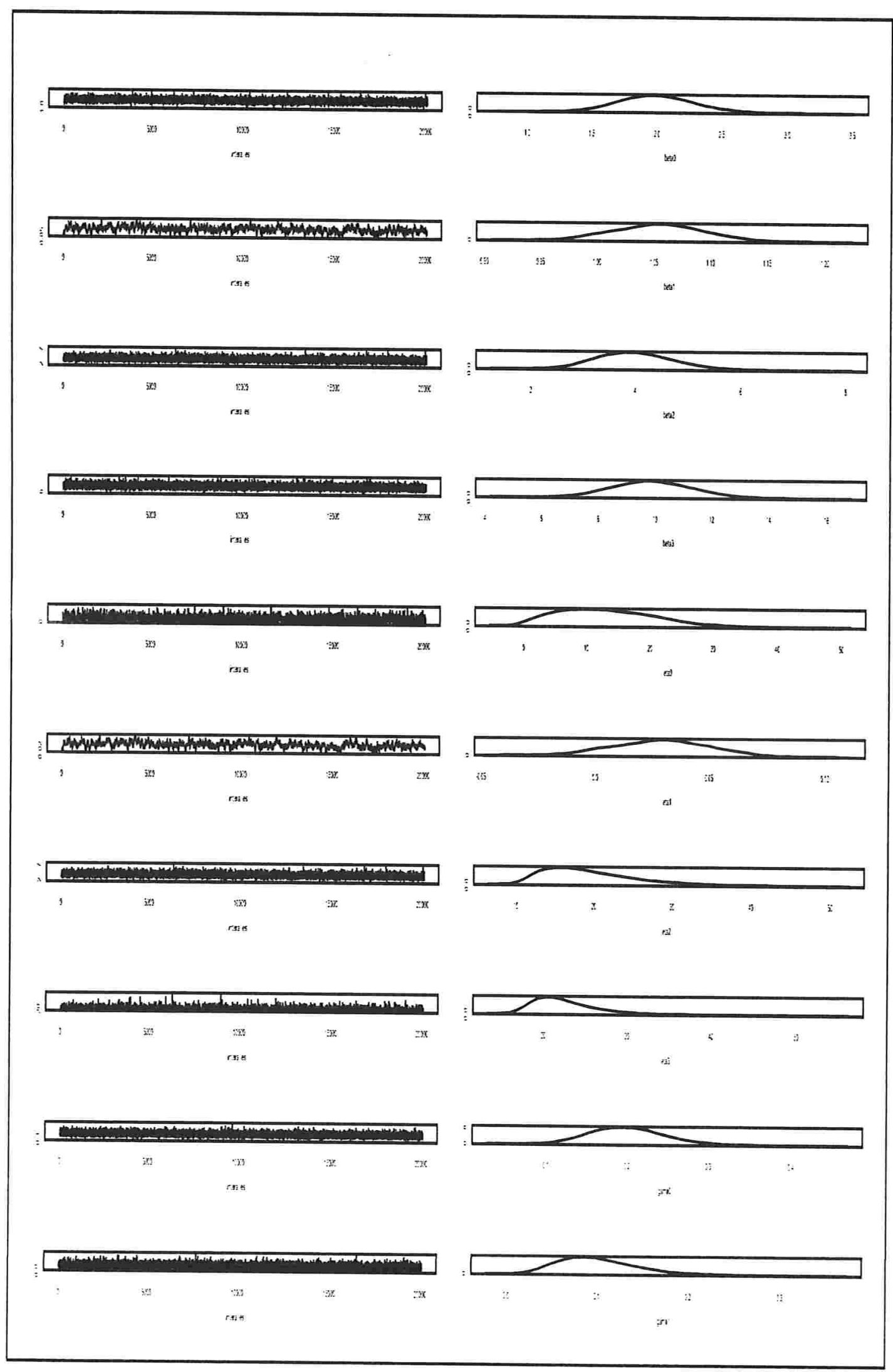

Figura 3.9: O traço e a Densidades a posteriori de $\beta_{0}, \beta_{1}, \beta_{2}, \xi_{3}, \xi_{0}, \xi_{1}, \xi_{2}, \xi_{3}, \gamma_{0}$ e $\gamma_{1}$. 


\section{Capítulo 4}

\section{Superposição de Processos de Poisson Não-homogeneos de Lei de Potências}

\subsection{Introdução}

Apresentamos neste capítulo, outra forma paramétrica especial para a função intensidade de superposição de dois PPNHs. Um processo Lei de Potência (PLP) é um PPNH que é considerado como um modelo de crescimento em confiabilidade. Um PLP é caracterizado pela taxa de ocorrência ou pela função intensidade dada por:

$$
\lambda_{j}(t \mid \underline{\beta})=\frac{\beta_{j}}{\theta_{j}}\left(\frac{t}{\theta_{j}}\right)^{\beta_{j}-1},
$$

para $j=1,2 ; \beta_{j}=\left(\theta_{j}, \beta_{j}\right)$.

Neste caso, a função do valor médio é dada por $m_{j}(t)=\left(\frac{t}{\theta_{j}}\right)^{\beta_{j}}, j=1,2$. Os parâmetros $\theta$ e $\beta$ são respectivamente chamados parâmetro de escala e forma do modelo PLP. Alguns casos especiais de modelos PLP quando o parâmetro $\beta$ assumi os seguintes valores:

(i) $\beta=1$ (Processo Homogêneo)

(ii) $3>1$ (Sistema em Deterioração)

(iii) $3<1$ ( . Iodelo de Crescimento em Confiabilidade)

Observamos que quando o paràmetro $3 \neq 1$, temos um processo de Poisson não Homogêneo. 


\subsection{Formulação da Superposição de dois PLP}

A modelagem de processo de Lei de Potência (MPLP) é frequentemente usado em modelos de falhas no sistemas de reparos, quando ambos tipos de renovações padrões estão presentes (ver por exemplo, Muralidharan, 2001). A MPLP permite que a taxa de falha pode ser afetada pelas falhas e pelo reparo.

Seja $M_{j}(t)$ o PLP que representa o número acumulado de falhas em $(0, t]$ para o $j$ ésimo processo componente com função intensidade $\lambda_{j}\left(t \mid \beta_{j}\right)$ assumindo uma superposição de $J$ PLP, cuja forma assumimos conhecida, mas incluíndo um vetor de parâmetros $\beta_{j}$ desconhecido. A função intensidade é dada em (1.11)

Seja $D_{t}$ o conjunto de dados observados até o tempo $t$ e $n$ épocas observadas:

$0<t_{1}<t_{2}<, \ldots, t_{n}<t$,(modelo de tempo truncado).

Considerando a função intensidade (4.1), com $j=1,2$ e a presença de dois PLP dependentes, a função de verossimilhança para $\underline{\beta}=\left(\underline{\beta_{1}}, \underline{\beta_{2}}\right)$ e $\rho$ dada em (1.18).

A seguir apresentamos alguns modelos PPNH de lei de potência com presença ou não de covariáveis.

\subsubsection{Modelo M1 (superposição de processos independentes sem a presença de covariáveis)}

Nesta seção consideramos a superposição de dois processos PLP independentes, as funções intensidades são dadas por

$$
\lambda_{1}(t)=\left(\frac{\beta_{1}}{\theta_{1}}\right)\left(\frac{t}{\theta_{1}}\right)^{\beta_{1}-1} \text { e } \lambda_{2}(t)=\left(\frac{\beta_{2}}{\theta_{2}}\right)\left(\frac{t}{\theta_{2}}\right)^{\beta_{2}-1}
$$

onde $\beta_{j}>0, \theta_{j}>0, j=1,2$.

A funções dos valores médios deste processo de superposição são dadas por

$$
m_{1}\left(t_{n}\right)=\left(\frac{t_{n}}{\theta_{1}}\right)^{\beta_{1}} \text { e } m_{2}\left(t_{n}\right)=\left(\frac{t_{n}}{\theta_{2}}\right)^{\beta_{2}}
$$

A função de verossimilhança (2.19) $\operatorname{com} \rho=0$ é dada por

$$
\begin{aligned}
L\left(\underline{B} \mid \mathfrak{D}_{\mathrm{t}}\right)= & \left\{\prod_{i=1}^{n}\left[\left(\frac{3_{1}}{\theta_{1}}\right)\left(\frac{t_{i}}{\theta_{1}}\right)^{\beta_{1}-1}+\left(\frac{3_{2}}{\theta_{2}}\right)\left(\frac{t_{i}}{\theta_{2}}\right)^{\beta_{2}-1}\right]\right\} \exp \left\{-\left[\left(\left(\frac{t_{n}}{\theta_{1}}\right)^{\beta_{1}}+\right.\right.\right. \\
& \left.\left.\left(\frac{t_{n}}{\theta_{2}}\right)^{\beta_{2}}\right]\right\}
\end{aligned}
$$

Denotemos o vetor de paràmetros deste modelo por $\underline{3}=\left(\theta_{1}, \theta_{2}, \beta_{1}, \beta_{2}\right)$. 


\subsubsection{Modelo M2 (superposição de processos dependentes sem a presença de covariáveis)}

Neste modelo onsideramos a superposição de dois PLP dependentes e a função intensidade a mesma do modelo M1, e o parâmetro de correlação $\rho$ constante. A função de verossimilhança (2.19) com $\rho$ constante é reescrita como,

$$
\begin{aligned}
L\left(\underline{\beta} \mid \mathfrak{D}_{\mathfrak{t}}\right) & =\prod_{i=1}^{n}\left[\left(\frac{\beta_{1}}{\theta_{1}}\right)\left(\frac{t_{i}}{\theta_{1}}\right)^{\beta_{1}-1}+\left(\frac{\beta_{2}}{\theta_{2}}\right)\left(\frac{t_{i}}{\theta_{2}}\right)^{\beta_{2}-1}\right]\left[1-\frac{\rho W\left(t_{i}\right)}{2}\right] \\
& \times \exp \left\{-\left[m_{1}\left(t_{n}\right)+m_{2}\left(t_{n}\right)-\rho\left(m_{1}\left(t_{n}\right) m_{2}\left(t_{n}\right)\right)^{1 / 2}\right]\right\}
\end{aligned}
$$

onde $W\left(t_{i}\right)$ é definido em (1.19) e $m_{1}\left(t_{n}\right)$ e $m_{2}\left(t_{n}\right)$ em (4.3). Denotamos o vetor de parâmetros deste modelo por $\underline{\beta}=\left(\theta_{1}, \theta_{2}, \beta_{1}, \beta_{2}, \rho\right)$.

\subsection{Superposição de dois PLP Dependentes na Pre- sença de uma Covariável}

Nesta seção vamos apresentar alguns casos especiais para o modelo de PLP considerando a função de intensidade do modelo M2 na presença de uma covariável, neste caso, afetando os parâmetros $\beta_{j}$ e $\theta_{j} \operatorname{com} j=1,2$.

\subsubsection{Modelo M3 (superposição de processos dependentes na presença de uma covariável)}

Neste modelo vamos assumir a superposição de dois PLP onde a covariável $x_{i}$ afeta o parâmetro $\theta_{j}$, mas não afeta o parâmetro $\beta_{j}$, na forma,

$$
\theta_{j}=\xi_{j}+\gamma_{j} x_{i} \quad j=1,2 . \quad i=1, \cdots, n
$$

As funções intensidades componentes destes modelo assumindo um efeito aditivo da covariável $x_{i}=1 \ldots n$, são cladlas por:

$$
\begin{aligned}
& \lambda_{1}(t)=\left(\frac{\beta_{1}}{\xi_{1}+\gamma_{1} \cdot x_{i}}\right)\left(\frac{t_{i}}{\xi_{1}+\gamma_{1} \cdot x_{i}}\right)^{\beta_{1}-1} \\
& \lambda_{2}(t)=\left(\frac{\beta_{2}}{\xi_{2}+\gamma_{2} \cdot x_{i}}\right)\left(\frac{t_{i}}{\xi_{2}+\gamma_{2} \cdot x_{i}}\right)^{s_{2}-1}
\end{aligned}
$$


Neste caso, as funções de valor médio componentes $m_{1}(t)$ e $m_{2}(t)$ são dadas por:

$$
m_{1}\left(t_{n}\right)=\left(\frac{t_{n}}{\xi_{1}+\gamma_{1} x_{i}}\right)^{\beta_{1}} \text { e } m_{2}\left(t_{n}\right)=\left(\frac{t_{n}}{\xi_{2}+\gamma_{2} x_{i}}\right)^{\beta_{2}}
$$

A função de verossimilhança (1.18) com $\rho$ constante é dada por:

$$
\begin{aligned}
L\left(\underline{\beta} \mid \mathfrak{D}_{\mathrm{t}}, \underline{x}_{i}\right)= & \prod_{i=1}^{n}\left[\left(\frac{\beta_{1}}{\xi_{1}+\gamma_{1} x_{i}}\right)\left(\frac{t_{i}}{\xi_{1}+\gamma_{1} x_{i}}\right)^{\beta_{1}-1}+\left(\frac{\beta_{2}}{\xi_{2}+\gamma_{2} x_{i}}\right)\left(\frac{t_{i}}{\xi_{2}+\gamma_{2} x_{i}}\right)^{\beta_{2}-1}\right][1- \\
& \left.\frac{\rho W\left(t_{i}\right)}{2}\right] \exp \left\{-\left[m_{1}\left(t_{n}\right)+m_{2}\left(t_{n}\right)-\rho\left(m_{1}\left(t_{n}\right) m_{2}\left(t_{n}\right)\right)^{1 / 2}\right]\right\}
\end{aligned}
$$

onde $W\left(t_{i}\right)$ é definido em (1.19) e $m_{1}\left(t_{n}\right)$ e $m_{2}\left(t_{n}\right)$ em (4.9). Denotamos o vetor de parâmetros deste modelo por $\underline{\beta}=\left(\beta_{1}, \beta_{2}, \xi_{1}, \xi_{2}, \gamma_{1}, \gamma_{2}, \rho\right)$.

\subsubsection{Modelo M4 (superposição de processos independentes na presença de uma covariável)}

Neste modelo, assumimos uma superposição de dois PLP independentes, isto é, temos $\rho=0 \mathrm{em}(4.10)$.

Considerando a mesma função de intensidade do Modelo M3 (4.7 e 4.8) a função de verossimilhança (4.10) com $\rho=0$ é rescrita como,

$$
\begin{aligned}
L\left(\underline{\beta} \mid \mathfrak{D}_{\mathrm{t}}, \underline{x}_{i}\right) & =\prod_{i=1}^{n}\left[\left(\frac{\beta_{1}}{\xi_{1}+\gamma_{1} x_{i}}\right)\left(\frac{t_{i}}{\xi_{1}+\gamma_{1} x_{i}}\right)^{\beta_{1}-1}+\left(\frac{\beta_{2}}{\xi_{2}+\gamma_{2} x_{i}}\right)\left(\frac{t_{i}}{\xi_{2}+\gamma_{2} x_{i}}\right)^{\beta_{2}-1}\right] \\
& \times \exp \left\{-\left[m_{1}\left(t_{n}\right)+m_{2}\left(t_{n}\right)\right]\right\}
\end{aligned}
$$

Denotamos o vetor de parâmetros deste modelo por $\underline{\beta}=\left(\beta_{1}, \beta_{2}, \xi_{1}, \xi_{2}, \gamma_{1}, \gamma_{2}\right)$.

\subsection{Análise Bayesiana}

\subsubsection{Modelo M1 (superposição de processos independentes sem a presença de covariáveis)}

Para uma análise Bayesiana desse modelo, consideramos as seguintes distribuições a priori para $\theta_{1}, \theta_{2}, \beta_{1}$ e $\beta_{2}$ :

$$
\begin{aligned}
& \theta_{1} \sim \Gamma\left(a_{1}, b_{1}\right) ; \operatorname{com} a_{1}, b_{1} \text { conhecidas } \\
& \theta_{2} \sim \Gamma\left(a_{2}, b_{2}\right) ; \operatorname{com} a_{2}, b_{2} \text { conhecidas } \\
& 3_{1} \sim \Gamma\left(c_{1}, d_{1}\right) ; \operatorname{com} c_{1}, d_{1} \text { conhecidas } \\
& 3_{2} \sim \Gamma\left(c_{2}, d_{2}\right) ; \operatorname{com} c_{2}, d_{2} \text { conhecidas }
\end{aligned}
$$


onde, $\Gamma(a, b)$ denota uma distribuição gama com média $a / b$ e variância $a / b^{2}$. Também assumimos independência a priori entre os parâmetros $\theta_{1}, \theta_{2}, \beta_{1}$ e $\beta_{2}$.

Existem muitas possibilidades para a escolha de distribuição priori. Neste capítulo a escolha da distribuição foi feita de acordo com o domínio do espaço paramétrico, isto é, distribuição gama para os parâmetros positivos $\left(\theta_{1}, \theta_{2}, \beta_{1}, \beta_{2}\right)$. Uma pequena análise de sensitividade foi feita para escolher os diferentes valores dos hiperparâmetros, os resultados são mostrados nos sumários à posteriori para os modelos, considerando dados simulados em exemplos no final deste capítulo.

Neste caso, as densidades a posteriori condicionais necessárias para o algoritmo Gibbs Sampling são dadas por:

(a) $\pi\left(\theta_{1} \mid \underline{\theta}_{\left(\theta_{1}\right)}, \underline{\nu}, \mathfrak{D}_{\mathfrak{t}}\right) \propto \theta_{1}^{a_{1}-1} e^{-b_{1} \theta_{1}} \psi_{1}(\underline{\beta})$

onde:

$$
\psi_{1}(\underline{\beta})=\exp \left\{-\left(\sum_{i=1}^{n} \nu_{i 1}\right) \beta_{1} \ln \left(\theta_{1}\right)\right\}
$$

(b) $\pi\left(\theta_{2} \mid \underline{\theta}_{\left(\theta_{2}\right)}, \underline{\nu}, \mathfrak{D}_{\mathrm{t}}\right) \propto \theta_{2}^{a_{2}-1} e^{-b_{2} \theta_{2}} \psi_{2}(\underline{\beta})$

onde:

$$
\psi_{2}(\underline{\beta})=\exp \left\{-\sum_{i=1}^{n} \nu_{i 2} \beta_{2} \ln \left(\theta_{2}\right)\right\}
$$

(c) $\pi\left(\beta_{1} \mid \underline{\theta}_{\left(\beta_{1}\right)}, \underline{\nu}, \mathfrak{D}_{\mathrm{t}}\right) \propto \beta^{c_{1}-1} e^{-d_{1} \beta} \psi_{4}(\underline{\beta})$

onde:

$$
\psi_{3}(\underline{\beta})=\exp \left\{\left(\sum_{i=1}^{n} \nu_{i 1}\right) \ln \left(\beta_{1}\right)+\left(\sum_{i=1}^{n} \nu_{i 1}\right) \beta_{1} \ln \left(\theta_{1}\right)+\left(\sum_{i=1}^{n} \nu_{i 1} \ln \left(t_{i}\right)\right), \beta_{1}\right\}
$$

(d) $\pi\left(\beta_{2} \mid \underline{\theta}_{\left(\beta_{2}\right)}, \underline{\nu}, \mathfrak{D}_{\mathfrak{t}}\right) \propto \beta^{c_{2}-1} e^{-d_{2} \beta} \psi_{4}(\underline{\beta})$

onde:

$$
u_{4}(\underline{3})=\exp \left\{\left(\sum_{i=1}^{n} \nu_{i 2}\right) \ln \left(3_{2}\right)+\sum_{i=1}^{n} \nu_{i 2} \ln \left(t_{i}\right) 3_{2}-\sum_{i=1}^{n} \nu_{i 2} \ln \left(\theta_{2}\right) 3_{2}\right\}
$$

As distribuições condicionais a posteriori dadas em (4.13) não apresentam uma distribuição paclrão conhecida, de modo que faremos uso do algoritmo de Metropolis-Hastings (Ver, Chib e Greenberg, 1995) para gerar amostras a posteriori dos paràmetros $\theta_{1}, \theta_{2}, 3_{1}$, e 3. 


\subsubsection{Modelo M2 (superposição de processos dependentes sem a presença de covariáveis)}

Para uma análise Bayesiana desse modelo,consideramos as seguintes distribuições a priori para $\theta_{1}, \theta_{2}, \beta_{1}, \beta_{2}$ e $\rho$ :

$$
\begin{aligned}
\theta_{1} & \sim \Gamma\left(a_{1}, b_{1}\right) ; \operatorname{com} a_{1}, b_{1} \text { conhecidas } \\
\theta_{2} & \sim \Gamma\left(a_{2}, b_{2}\right) ; \operatorname{com} a_{2}, b_{2} \text { conhecidas } \\
\beta_{1} & \sim \Gamma\left(c_{1}, d_{1}\right) ; \operatorname{com} c_{1}, d_{1} \text { conhecidas } \\
\beta_{2} & \sim \Gamma\left(c_{2}, d_{2}\right) ; \operatorname{com} c_{2}, d_{2} \text { conhecidas } \\
\rho & \sim U\left(e_{1}, f_{1}\right) ; \operatorname{com} e_{1}, f_{1} \text { conhecidas }
\end{aligned}
$$

onde, $\rho$ é definida no intervalo $(0,1)$, isto é, $0 \leq \rho \leq 1$. A escolha das distribuições a priori Uniforme para $\rho$ devem levar em consideração essa restrição existente, isto é, $0 \leq \rho \leq 1$.

Neste caso, as densidades a posteriori condicionais necessárias para o algoritmo Gibbs Sampling são dadas por:

(a) $\pi\left(\theta_{1} \mid \underline{\theta}_{\left(\theta_{1}\right)}, \underline{\nu}, \mathfrak{D}_{\mathfrak{t}}\right) \propto \theta_{1}^{a_{1}-1} e^{-b_{1} \theta_{1}} \psi_{1}(\underline{\beta})$

onde:

$$
\begin{aligned}
\psi_{1}(\underline{\beta}) & =\exp \left\{-\left(\sum_{i=1}^{n} \nu_{i 1}\right) \beta_{1} \ln \left(\theta_{1}\right)+\sum_{i=1}^{n} \nu_{i 1} \ln \left(1-\frac{\rho A_{1 i}(\underline{\beta})}{2}\right)+\sum_{i=1}^{n} \nu_{i 2} \ln \left(1-\frac{\rho A_{2 i}(\underline{\beta})}{2}\right)\right. \\
& \left.-\left(\frac{t_{n}}{\theta_{1}}\right)^{\beta_{1}}+\rho\left[\left(\frac{t n}{\theta_{1}}\right)^{\beta_{1}}\left(\frac{t n}{\theta_{2}}\right)^{\beta_{2}}\right]^{1 / 2}\right\}
\end{aligned}
$$

Para simplificar denotamos

$$
A_{1 i}\left(\theta_{1}, \theta_{2}, \beta_{1}, \beta_{2}\right) \text { e } A_{2 i}\left(\theta_{1}, \theta_{2}, \beta_{1}, \beta_{2}\right)
$$

por $A_{1 i}(\underline{\beta})$ e $A_{2 i}(\underline{\beta})$, onde,

(i) $A_{1 i}(\underline{3})=\left[\frac{m_{2}\left(t_{i}\right)}{m_{1}\left(t_{i}\right)}\right]^{1 / 2}=\sqrt{\frac{t_{n}^{j_{2}}}{t_{n}^{j_{1}}} \frac{\theta_{1}^{j_{1}}}{\theta_{2}^{j_{2}}}}$

(ii) $A_{2 i}(\underline{\beta})=\left[\frac{m_{1}\left(t_{i}\right)}{m_{2}\left(t_{i}\right)}\right]^{1 / 2}=\sqrt{\frac{t_{n}^{3_{1}}}{t_{n}^{j_{2}}} \frac{\theta_{2}^{3_{2}}}{\theta_{1}^{3_{1}}}}$

(b) $\pi\left(\theta_{2} \mid \underline{\theta}_{\left(\theta_{2}\right)}, \underline{\nu}, \mathfrak{D}_{\mathrm{t}}\right) \propto \theta_{2}^{a_{2}-1} e^{-b_{2} \theta_{2}} \iota_{2}(\underline{\beta})$ 
onde:

$$
\begin{aligned}
& \begin{aligned}
\psi_{2}(\underline{\beta}) & =\exp \left\{\sum_{i=1}^{n} \nu_{i 1} \ln \left(1-\frac{\rho A_{1 i}(\underline{\beta})}{2}\right)-\sum_{i=1}^{n} \nu_{i 2} \beta_{2} \ln \left(\theta_{2}\right)+\sum_{i=1}^{n} \nu_{i 2} \ln \left(1-\frac{\rho A_{2 i}(\underline{\beta})}{2}\right)\right. \\
& \left.-\left(\frac{t_{n}}{\theta_{2}}\right)^{\beta_{2}}+\rho\left[\left(\frac{t n}{\theta_{1}}\right)^{\beta_{1}}\left(\frac{t n}{\theta_{2}}\right)^{\beta_{2}}\right]^{1 / 2}\right\}
\end{aligned} \\
& \text { (c) } \pi\left(\beta_{1} \mid \underline{\theta}_{\left(\beta_{1}\right)}, \underline{\nu}, \mathfrak{D}_{\mathrm{t}}\right) \propto \beta_{1}^{c_{1}-1} e^{-d_{1} \beta_{1}} \psi_{3}(\underline{\beta})
\end{aligned}
$$

onde:

$$
\begin{aligned}
\psi_{3}(\underline{\beta})= & \exp \left\{\left(\sum_{i=1}^{n} \nu_{i 1}\right) \ln \left(\beta_{1}\right)+\sum_{i=1}^{n} \nu_{i 1} \ln \left(t_{i}\right) \beta_{1}+\sum_{i=1}^{n} \nu_{i 1} \ln \left(\theta_{1}\right) \beta_{1}+\sum_{i=1}^{n} \nu_{i 1} \ln \left(1-\frac{\rho A_{1 i}(\underline{\beta})}{2}\right)+\right. \\
& \left.\sum_{i=1}^{n} \nu_{i 2} \ln \left(1-\frac{\rho A_{2 i}(\underline{\beta})}{2}\right)-\left(\frac{t_{n}}{\theta_{1}}\right)^{\beta_{1}}+\rho\left[\left(\frac{t n}{\theta_{1}}\right)^{\beta_{1}}\left(\frac{t n}{\theta_{2}}\right)^{\beta_{2}}\right]^{1 / 2}\right\}
\end{aligned}
$$

(d) $\pi\left(\beta_{2} \mid \underline{\theta}_{\left(\beta_{2}\right)}, \underline{\nu}, \mathfrak{D}_{\mathrm{t}}\right) \propto \beta_{2}^{c_{2}-1} e^{-d_{2} \beta_{2}} \psi_{3}(\underline{\beta})$

onde:

$$
\begin{aligned}
\psi_{4}(\underline{\beta})= & \exp \left\{-\left(\sum_{i=1}^{n} \nu_{i 1} \ln \left(1-\frac{\rho A_{1 i}(\underline{\beta})}{2}\right)+\sum_{i=1}^{n} \nu_{i 1} \ln \left(\beta_{2}\right)+\sum_{i=1}^{n} \nu_{i 2} \ln \left(t_{i}\right) \beta_{2}-\right.\right. \\
& \left.\sum_{i=1}^{n} \nu_{i 2} \ln \left(\theta_{2}\right) \beta_{2}+\sum_{i=1}^{n} \nu_{i 2} \ln \left(1-\frac{\rho A_{2 i}(\underline{\beta})}{2}\right)-\left(\frac{t_{n}}{\theta_{2}}\right)^{\beta_{2}}+\rho\left[\left(\frac{t n}{\theta_{1}}\right)^{\beta_{1}}\left(\frac{t n}{\theta_{2}}\right)^{\beta_{2}}\right]^{1 / 2}\right\}
\end{aligned}
$$

(e) $\pi\left(\rho \mid \underline{\theta}_{(\rho)}, \underline{\nu}, \mathfrak{D}_{\mathfrak{t}}\right) \propto \frac{1}{f_{1}-e_{1}} \psi_{5}(\underline{\beta})$

onde:

$$
\begin{aligned}
\psi_{5}(\underline{\beta})= & \exp \left\{\sum_{i=1}^{n} \nu_{i 1} \ln \left(1-\frac{\rho A_{1 i}(\underline{\beta})}{2}\right)+\sum_{i=1}^{n} \nu_{i 2} \ln \left(1-\frac{\rho A_{2 i}(\underline{\beta})}{2}\right)+\right. \\
& \left.\rho\left[\left(\frac{t n}{\theta_{1}}\right)^{\beta_{1}}\left(\frac{t n}{\theta_{2}}\right)^{\beta_{2}}\right]^{1 / 2}\right\}
\end{aligned}
$$

As distribuições condicionais a posteriori dadas em (4.15) não apresentam uma distribuição padrão conhecida, de modo que faremos uso do algoritmo de Metropolis-Hastings (Ver, Chib e Greenberg, 1995) para gerar amostras a posteriori clos parâmetros $\theta_{1}, \theta_{2}, \beta_{1}, \zeta_{2}$ e $\rho$.

De forma similar os cálculos das distribuições condicionais a posteriori foram feitos para a análise dos demais modelos na presença ou não de mua covariável. 


\subsection{Aplicação Numérica}

Nesta seção, consideramos um conjunto de dados simulados apresentado na Tabela 4.1 a partir da superposição de processos PLP (Processo Lei de Potência) com função intensidade

$$
\lambda(t)=\frac{\beta}{\theta_{1}}\left(\frac{t}{\theta_{1}}\right)^{\beta-1}+\frac{\beta}{\theta_{2}}\left(\frac{t}{\theta_{2}}\right)^{\beta-1},
$$

para os valores fixados $\theta_{1}=2, \theta_{2}=8$ e $\beta=\beta_{1}=\beta_{2}=0.5 ; 20$ observações foram geradas com ponto de mudança $\tau=10$. Os resultados da análise dos modelos M1, M2 , M3 e M4 foram obtidos apartir de métodos MCMC implementado no software WINBUGS (Spiegelhalter et al., 1999) após descartar as 4000 primeiras observações (burnin). As séries de valores simulados foram avaliadas usando os métodos de convergência de Geweke (1992), Raftery \& Lewis (1992) e Gelman \& Rubin (1992), respectivamente, implementados no software CODA (Best et al., 1997). Os resultados para as cadeias geradas foram satisfatórias, e isso pode ser visto graficamente na Figuras a seguir onde apresentamos o traço e a densidade dos parâmetros de interesses.

Os sumários a posteriori para os parâmetros de interesse de cada modelo apresentado neste capítulo são apresentados nas Tabelas 4.2 a 4.4 .

Tabela 4.1: Dados gerados a partir de um processo PLP.

\begin{tabular}{c|c|c|c|c|c|c|c}
\hline \hline$t_{i}$ & $x_{i}$ & $t_{i}$ & $x_{i}$ & $t_{i}$ & $x_{i}$ & $t_{i}$ & $x_{i}$ \\
\hline 0,1235 & 0 & 19,1212 & 1 & 207,9458 & 1 & 478,9752 & 1 \\
0.6658 & 0 & 29,9314 & 1 & 211,2891 & 1 & 539,9856 & 1 \\
1,2409 & 0 & 31,4086 & 1 & 290,9619 & 1 & 640,7768 & 1 \\
3,8394 & 0 & 49.6908 & 1 & 299,0633 & 1 & 1031,9686 & 1 \\
7,8674 & 0 & 50,2999 & 1 & 337,9812 & 1 & 1047,1765 & 1 \\
\hline \hline
\end{tabular}

\subsubsection{Modelo M1 (superposição de processos independentes sem a presença de uma covariável)}

Neste caso, vamos assumir uma superposição de dois processos PLP considerando as distribuições a priori dadas para o modelo MI1 (4.13), onde fixamos alguns valores para os hiperparàmetros (Vrs Hip). Os resultados a posteriori estão sumarizados na Tabela 4.2, onde estão apresentadas características tais como média a posteriori, desvio-padrão (DP) e intervalos de credibilidade de $95 \%$ para os parametros de interesse. Os resultados para 
as cadeias geradas foram satisfatórios, e isso pode ser visto graficamente na Figura 4.1 que apresenta o traço e a densidade dos parâmetros de interesse.

Tabela 4.2: Resumos a posteriori para o modelo M1.

\begin{tabular}{c|c|c|c|c|c}
\hline Parâmetro & Vrs. Hip & Média & D.P & Interv. de Credibilidade & $\hat{R}$ \\
\hline \hline \multirow{6}{*}{$\theta_{1}$} & $\Gamma(80 ; 40)$ & 2,086 & 0,2255 & $(1,668 ; 2,549)$ & 1,005 \\
\cline { 2 - 6 } & $\Gamma(400,200)$ & 2,017 & 0,1001 & $(1,826 ; 2,218)$ & 1,014 \\
\cline { 2 - 6 } & $\Gamma(90 ; 45)$ & 2,085 & 0,2108 & $(1,692 ; 2,516)$ & 1,037 \\
\hline \multirow{3}{*}{$\theta_{2}$} & $\Gamma(640 ; 80)$ & 8,02 & 0,3179 & $(7,408 ; 8,655)$ & 1,004 \\
\cline { 2 - 6 } & $\Gamma(600,75)$ & 8,023 & 0,3271 & $(7,394 ; 8,677)$ & 1,006 \\
\cline { 2 - 6 } & $\Gamma(120 ; 15)$ & 8,127 & 0,7306 & $(6,756 ; 9,622)$ & 1,002 \\
\hline \multirow{3}{*}{$\beta_{1}$} & $\Gamma(80 ; 1600)$ & 0,4831 & 0,01567 & $(0,4529 ; 0,5141)$ & 1,002 \\
\cline { 2 - 6 } & $\Gamma(900,1800)$ & 0,4845 & 0,01482 & $(0,4559 ; 0,514)$ & 1,001 \\
\cline { 2 - 6 } & $\Gamma(1300 ; 2600)$ & 0,4885 & 0,01275 & $(0,4638 ; 0,5136)$ & 1,004 \\
\hline \multirow{3}{*}{$\beta_{2}$} & $\Gamma(200 ; 400)$ & 0,4727 & 0,03044 & $(0,4143 ; 0,5336)$ & 1,001 \\
\cline { 2 - 6 } & $\Gamma(150 ; 300)$ & 0,4851 & 0,03403 & $(0,3997 ; 0,5332)$ & 1,003 \\
\cline { 2 - 6 } & $\Gamma(550 ; 1100)$ & 0,4883 & 0,01998 & $(0,4501 ; 0,5279)$ & 1,005 \\
\hline \hline
\end{tabular}

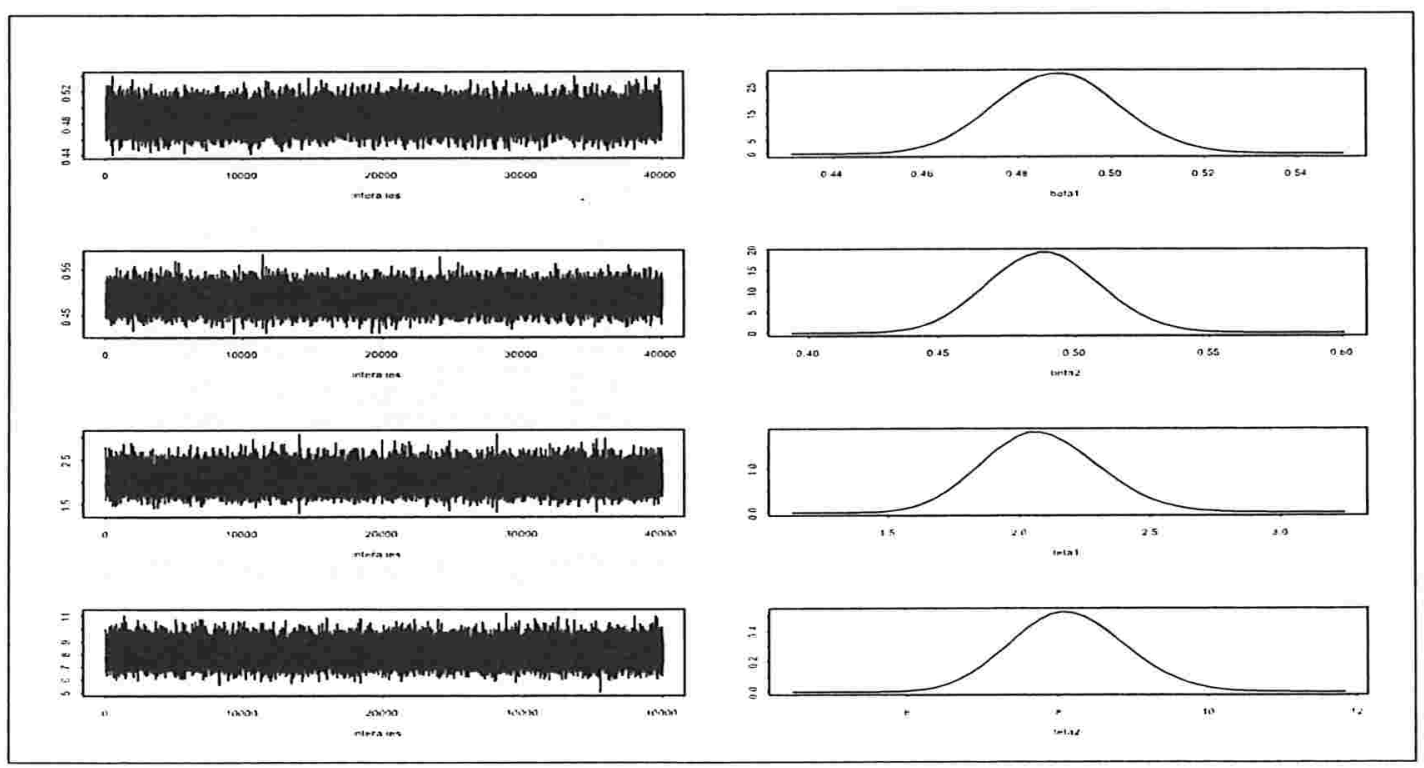

Figura 4.1: O traço e a Densidade a posteriori de $3_{1}, 3_{2}, \theta_{1}$ e $\theta_{2}$.

\subsubsection{Modelo M2 (superposição de processos dependentes sem a presença de covariáveis)}

Neste caso. vamos considerar a superposição de processos PLP, dependentes onde a covariável não afeta o paràmetro de correlação $\rho$ e nem as intensidades individuais para cada componente. 
Considerando as distribuições a priori do modelo M2 (4.14), temos na Tabela 4.3, os resumos a posteriori correspondentes aos parâmetros $\theta_{1}, \theta_{2}, \beta_{1}, \beta_{2}$ e $\rho$. Com valores diferentes dos hiperparâmetros as médias a posteriori são próximas para cada parâmetros do modelo, variando apenas os erros-padrão e os resultados para as cadeias geradas foram satisfatórios, e isso pode ser visto graficamente na Figura 4.2 que apresenta o traço e a densidade dos parâmetros de interesse. Também temos $\hat{R}$ menores do que 1,1 para todos os casos, o que indica convergência do algoritmo.

Tabela 4.3: Resumos a posteriori para o modelo M2.

\begin{tabular}{c|c|c|c|c|c}
\hline Parâmetro & Vrs. Hip & Média & D.P & Interv. de Credibilidade & $\hat{R}$ \\
\hline \hline \multirow{6}{*}{$\theta_{1}$} & $\Gamma(2400 ; 1200)$ & 2,001 & 0,04084 & $(1,922 ; 2,082)$ & 1,008 \\
\cline { 2 - 6 } & $\Gamma(400,200)$ & 2,005 & 0,1005 & $(1,813 ; 2,207)$ & 1,011 \\
\cline { 2 - 6 } & $\Gamma(90 ; 45)$ & 2,022 & 0,2094 & $(1,634 ; 2,453)$ & 1,005 \\
\hline \multirow{3}{*}{$\theta_{2}$} & $\Gamma(4800 ; 600)$ & 8,001 & 0,1159 & $(7,776 ; 8,231)$ & 1,001 \\
\cline { 2 - 6 } & $\Gamma(600,75)$ & 8,023 & 0,3271 & $(7,394 ; 8,677)$ & 1,006 \\
\cline { 2 - 6 } & $\Gamma(120 ; 15)$ & 8,034 & 0,7317 & $(6,666 ; 9,541)$ & 1,001 \\
\hline \multirow{3}{*}{$\beta_{1}$} & $\Gamma(810 ; 1620)$ & 0,4921 & 0,01635 & $(0,4602 ; 0,5243)$ & 1,002 \\
\cline { 2 - 6 } & $\Gamma(900,1800)$ & 0,4927 & 0,01571 & $(0,4623 ; 0,5238)$ & 1,004 \\
\cline { 2 - 6 } & $\Gamma(1300 ; 2600)$ & 0,44949 & 0,01329 & $(0,469 ; 0,512)$ & 1,005 \\
\hline \multirow{3}{*}{$\beta_{2}$} & $\Gamma(550 ; 1100)$ & 0,4958 & 0,02097 & $(0,4554 ; 0,5381)$ & 1,015 \\
\cline { 2 - 6 } & $\Gamma(150 ; 300)$ & 0,4855 & 0,03801 & $(0,4128 ; 0,5822)$ & 1,002 \\
\cline { 2 - 6 } & $\Gamma(450 ; 900)$ & 0,4949 & 0,02287 & $(0,451 ; 0,5406)$ & 1,009 \\
\hline \multirow{2}{*}{$\rho$} & $U(-1 ; 1)$ & 0,6584 & 0,2512 & $(0,05418 ; 0,9845)$ & 1,007 \\
\cline { 2 - 6 } & $U(-1 ; 1)$ & 0,6429 & 0,266 & $(-0,001543 ; 0,9839)$ & 1,003 \\
\cline { 2 - 6 } & $U(-1 ; 1)$ & 0,666 & 0,2482 & $(0,06166 ; 0,9846)$ & 1,037 \\
\hline \hline
\end{tabular}

\subsubsection{Modelo M3 (superposição de processos dependentes na presença de uma covariável)}

Neste caso, vamos considerar a superposição processos PLP dependentes onde a covariável $x_{i}$ afeta o parâmetro $\theta_{j}, j=1,2$ mas não afeta o parâmetro de correlação $\rho$.

Considerando as seguintes distribuições a priori do modelo M3

$$
\begin{aligned}
& 3_{1} \sim \Gamma\left(a_{1}, b_{1}\right) ; \operatorname{com} a_{1}, b_{1} \text { conheciclas } \\
& 3_{2} \sim \Gamma\left(a_{2}, b_{2}\right) ; \operatorname{com} a_{2}, b_{2} \text { conhecidas } \\
& \xi_{1} \sim \Gamma\left(c_{1}, d_{1}\right) ; \operatorname{com} c_{1}, d_{1} \text { conhecidas }
\end{aligned}
$$




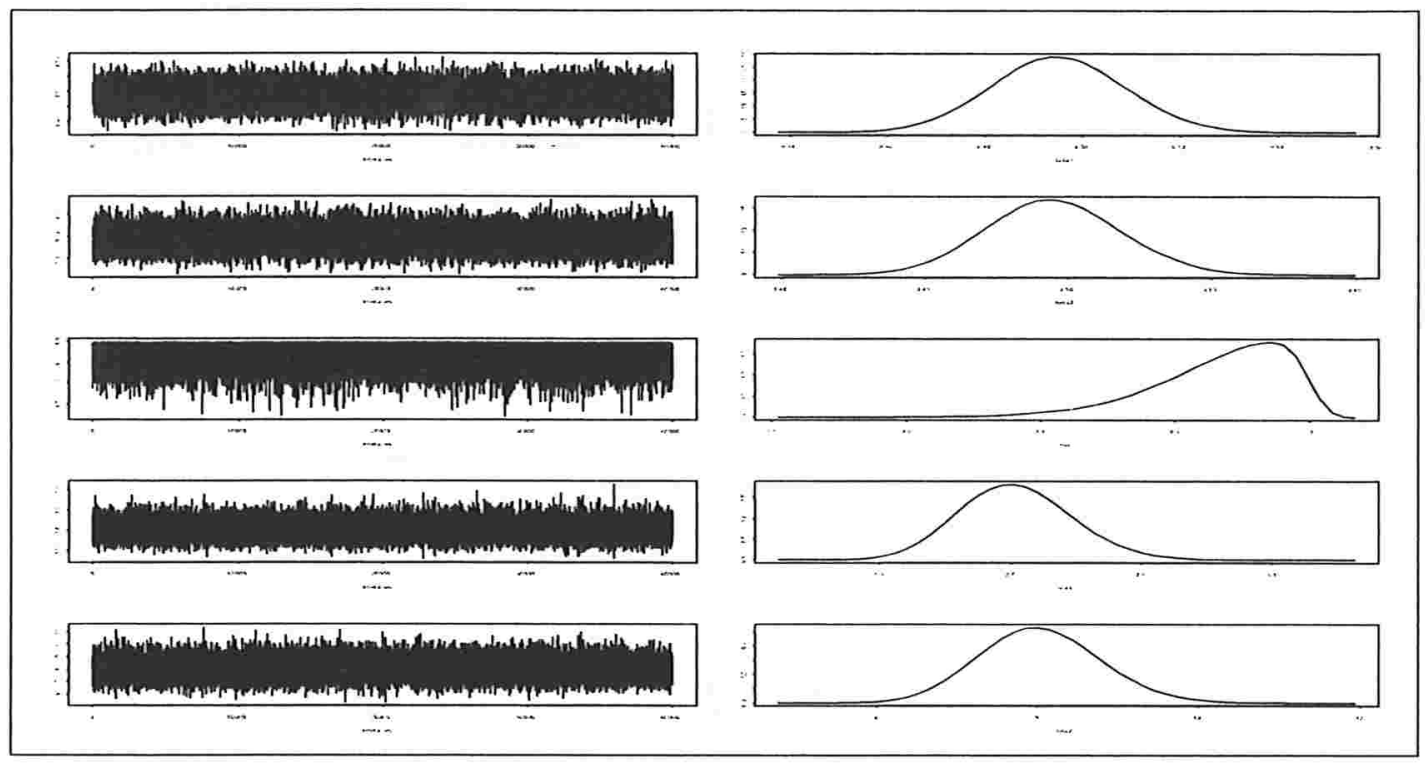

Figura 4.2: O traço e a Densidade a posteriori de $\beta_{1}, \beta_{2}, \rho, \theta_{1}$ e $\theta_{2}$

$$
\begin{aligned}
\xi_{2} & \sim \Gamma\left(c_{2}, d_{2}\right) ; \operatorname{com} c_{2}, d_{2} \text { conhecidas } \\
\gamma_{1} & \sim \Gamma\left(g_{1}, h_{1}\right) ; \operatorname{com} g_{1}, h_{1} \text { conhecidas } \\
\gamma_{2} & \sim \Gamma\left(g_{2}, h_{2}\right) ; \operatorname{com} g_{2}, h_{2} \text { conhecidas } \\
\rho & \sim U\left(e_{1}, f_{1}\right) ; \text { com } e_{1}, f_{1} \text { conhecidas }
\end{aligned}
$$

\begin{tabular}{|c|c|c|c|c|c|}
\hline Parâmetro & Vrs. Hip & Média & D.P & Interv. de Credibilidade & $\hat{R}$ \\
\hline \multirow[t]{2}{*}{$\beta_{1}$} & 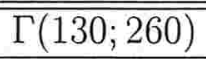 & 0,4627 & 0,0347 & $(0.395 ; 0,5302)$ & 1,002 \\
\hline & $\Gamma(200,400)$ & 0,4721 & 0,0296 & $(0,4146 ; 0,5304)$ & 1,027 \\
\hline \multirow[t]{2}{*}{$\beta_{2}$} & $\Gamma(45 ; 90)$ & 0,4644 & 0,05942 & $(0,3512 ; 0,5825)$ & 1,008 \\
\hline & $\Gamma(100,200)$ & 0,4817 & 0,04453 & $(0,3972 ; 0,5719)$ & 1,003 \\
\hline \multirow[t]{2}{*}{$\xi_{1}$} & $\Gamma(100 ; 50)$ & 2.018 & 0,2016 & $(1,643: 2.433)$ & 1,005 \\
\hline & $\Gamma(20,10)$ & 2.117 & 0,4541 & $(1.324 ; 3.1)$ & 1,001 \\
\hline \multirow[t]{2}{*}{$\xi_{2}$} & $\Gamma(120 ; 60)$ & 1.986 & 0,1819 & $(1.647: 2.359)$ & 1.003 \\
\hline & $\Gamma(40,20)$ & 1.961 & 0,3185 & $(1.39: 2.632)$ & 1,003 \\
\hline \multirow[t]{2}{*}{$\gamma_{1}$} & $\Gamma(2 ; 10)$ & 0.2236 & 0,157 & $(0.02709 ; 0,6171)$ & 1,018 \\
\hline & $\Gamma(4: 40)$ & 0,1032 & 0,0515 & $(0,02785 ; 0,2263)$ & 1,004 \\
\hline \multirow[t]{2}{*}{12} & $\Gamma(6: 10)$ & 6,014 & 0.3165 & $(5.403 ; 6,634)$ & 1.017 \\
\hline & $\Gamma(660 ; 110)$ & 6,007 & 0.2347 & $(5.555 ; 6,479)$ & 1.003 \\
\hline \multirow[t]{2}{*}{$\rho$} & $U(-1 ; 1)$ & 0,3761 & 0,404 & $(-0.5763: 0.9588)$ & 1,022 \\
\hline & $U(-1 ; 1)$ & 0,4693 & 0.3556 & $(-0.371: 0.9677)$ & 1.013 \\
\hline
\end{tabular}

Na Tabela 4.4, apresentamos resumos a posteriori correspondentes aos parâmetros $\beta_{1}, \beta_{2}, \xi_{1}, \xi_{1}, \gamma_{1}, \gamma_{2}$ e $\rho$, com diferentes valores para os hiperparâmetros.

Tabela 4.4: Resumos a posteriori para o modelo M3. 


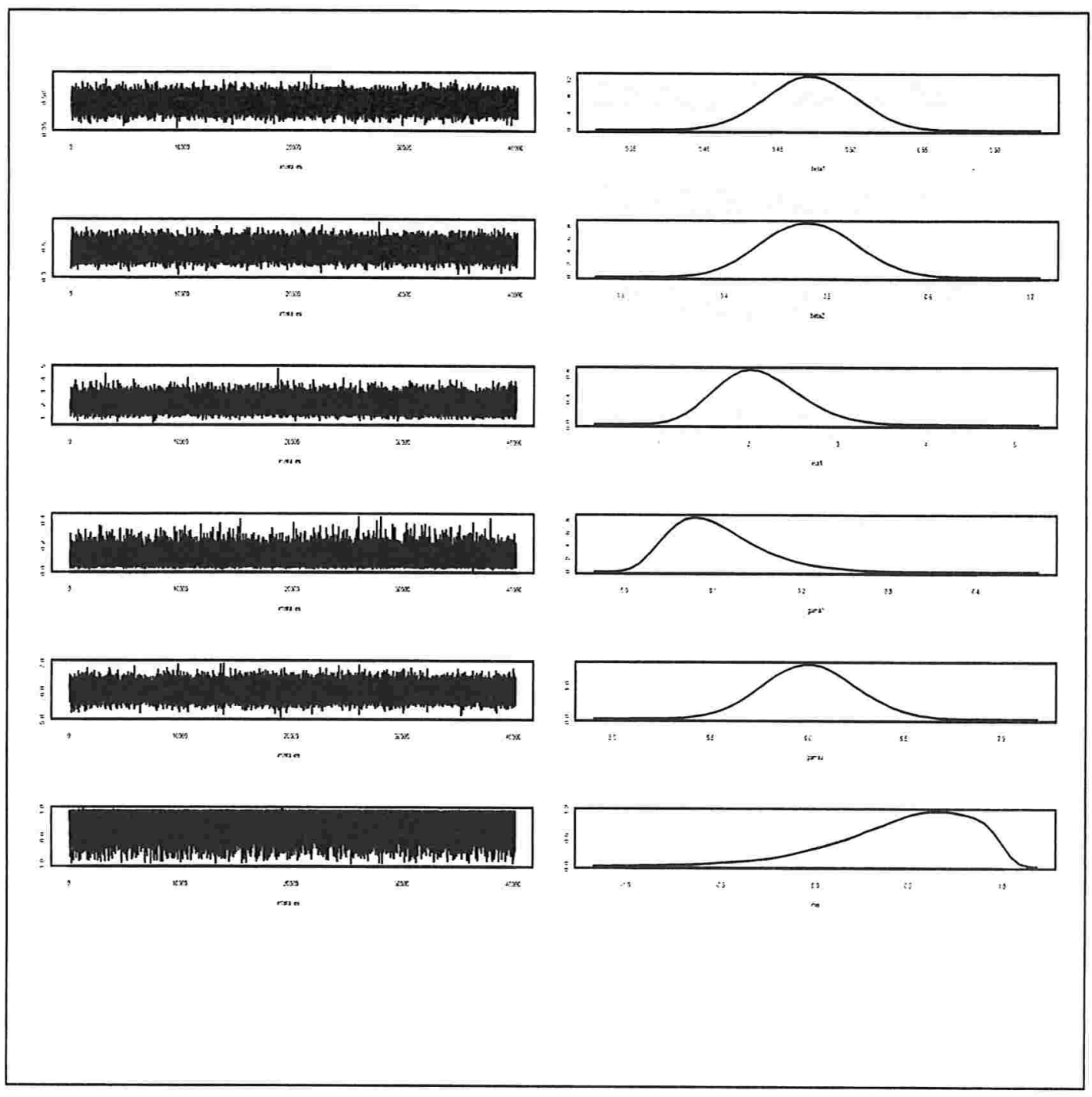

Figura 4.3: O traço e a Densidade a posteriori de $\beta_{1}, \beta_{2}, \rho, \theta_{1}$ e $\theta_{2}$

\subsubsection{Modelo M4 (superposição de processos independentes na presença de uma covariável)}

Neste caso, vamos considerar a superposição processos PLP independentes onde a covariável $x_{i}$ afeta o parâmetro $\theta_{j}$ em 4.6 . 
Considerando as seguintes distribuições a priori do modelo M4

$$
\begin{aligned}
\beta_{1} & \sim \Gamma\left(a_{1}, b_{1}\right) ; \operatorname{com} a_{1}, b_{1} \text { conhecidas } \\
\beta_{2} & \sim \Gamma\left(a_{2}, b_{2}\right) ; \operatorname{com} a_{2}, b_{2} \text { conhecidas } \\
\xi_{1} & \sim \Gamma\left(c_{1}, d_{1}\right) ; \operatorname{com} c_{1}, d_{1} \text { conhecidas } \\
\xi_{2} & \sim \Gamma\left(c_{2}, d_{2}\right) ; \operatorname{com} c_{2}, d_{2} \text { conhecidas } \\
\gamma_{1} & \sim \Gamma\left(g_{1}, h_{1}\right) ; \operatorname{com} g_{1}, h_{1} \text { conhecidas } \\
\gamma_{2} & \sim \Gamma\left(g_{2}, h_{2}\right) ; \operatorname{com} g_{2}, h_{2} \text { conhecidas }
\end{aligned}
$$

Na Tabela 4.5, apresentamos resumos a posteriori correspondentes aos parâmetros $\beta_{1}, \beta_{2}, \xi_{1}, \xi_{2}, \gamma_{1}$, e $\gamma_{2}$, com diferentes valores para os hiperparâmetros.

Tabela 4.5: Resumos a posteriori para o modelo M4.

\begin{tabular}{c|c|c|c|c|c}
\hline Parâmetro & Vrs. Hip & Média & D.P & Interv. de Credibilidade & $\hat{R}$ \\
\hline \hline \multirow{2}{*}{$\beta_{1}$} & $\Gamma(130 ; 260)$ & 0,4451 & 0,03065 & $(0,3854 ; 0,5057)$ & 1,014 \\
\cline { 2 - 6 } & $\Gamma(400,800)$ & 0,4722 & 0,02067 & $(0,4326 ; 0,5133)$ & 1,011 \\
\hline \multirow{2}{*}{$\beta_{2}$} & $\Gamma(100 ; 200)$ & 0,4722 & 0,03448 & $(0,4062 ; 0,541)$ & 1,002 \\
\cline { 2 - 7 } & $\Gamma(150,300)$ & 0,4817 & 0,04453 & $(0,3972 ; 0,5719)$ & 1,003 \\
\hline \multirow{2}{*}{$\xi_{1}$} & $\Gamma(20 ; 10)$ & 2,174 & 0.458 & $(1,369 ; 3,161)$ & 1,006 \\
\cline { 2 - 7 } & $\Gamma(40,20)$ & 2,14 & 0,3188 & $(1,561 ; 2,805)$ & $1,009)$ \\
\hline \multirow{2}{*}{$\xi_{2}$} & $\Gamma(40 ; 20)$ & 1,96 & 0,3139 & $(1,393 ; 2,619)$ & 1,003 \\
\cline { 2 - 7 } & $\Gamma(80,40)$ & 1,984 & 0,2234 & $(1,572 ; 2,45)$ & 1,004 \\
\hline \multirow{2}{*}{$\gamma_{1}$} & $\Gamma(4 ; 40)$ & 0,1035 & 0,05162 & $(0,02826 ; 0,2267)$ & 1,002 \\
\cline { 2 - 6 } & $\Gamma(5 ; 50)$ & 0,1044 & 0,04619 & $(0,03425 ; 0,2124)$ & 1,001 \\
\hline \multirow{2}{*}{$\gamma_{2}$} & $\Gamma(6 ; 1)$ & 7,433 & 2,697 & $(3,107 ; 13,56)$ & 1,001 \\
\cline { 2 - 6 } & $\Gamma(120 ; 20)$ & 6,088 & 0,5532 & $(5,05 ; 7,22)$ & 1,017 \\
\hline \hline
\end{tabular}

\subsection{Seleção do modelo}

Na seção 1.3.2 apresentamos os procedimentos para selecionar qual é o melhor modelo que ajusta ao conjuntos dos dados. Utilizamos o método DIC implementado no pacote WinBugs (ver Spiegelhater et al., 2002), assim temos os resultados na Tabela 4.6 que os modelos $\mathrm{M} 3$ e $\mathrm{M} 4$ indicam os melhores ajustes. 


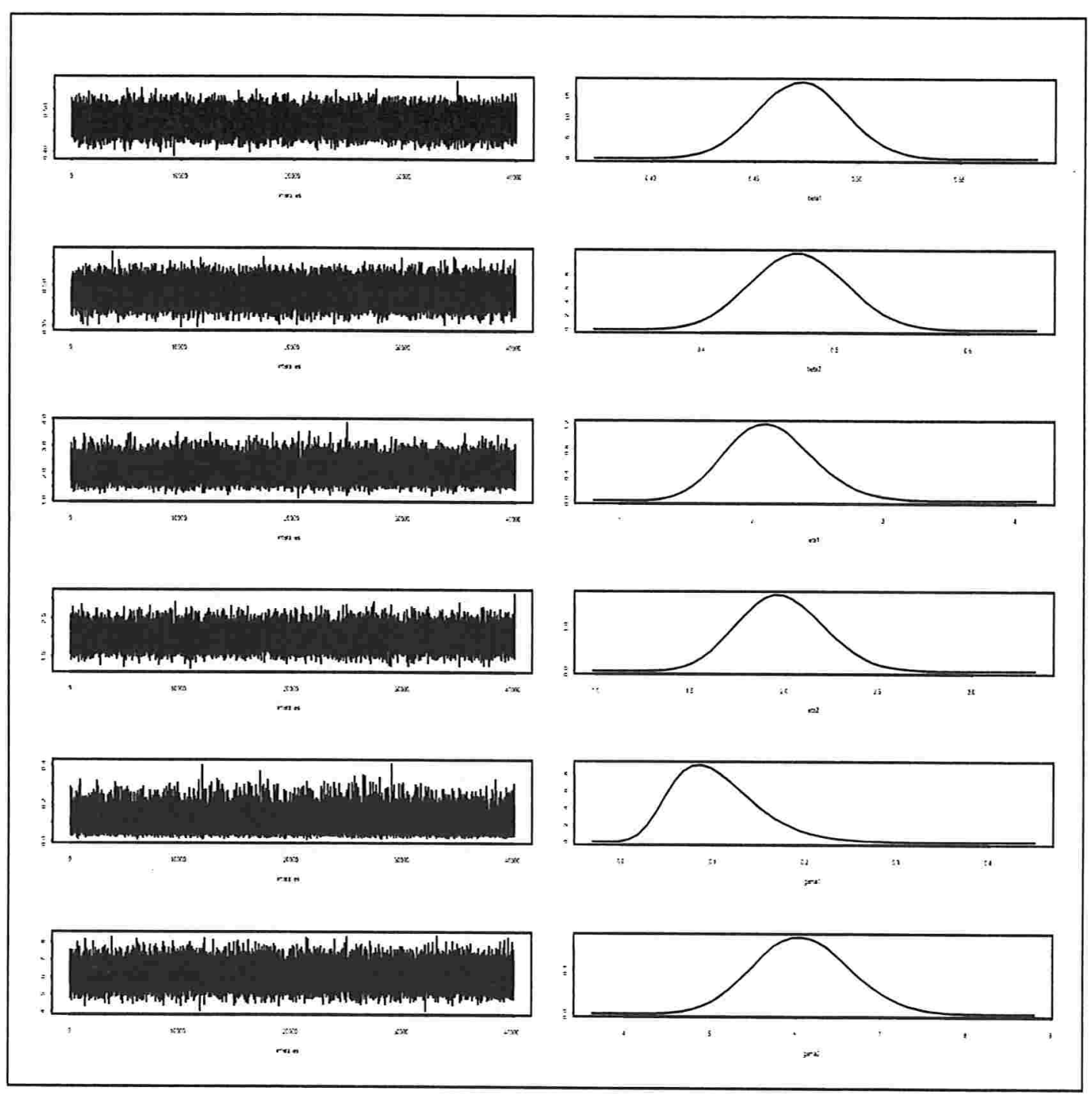

Figura 4.4: $\mathrm{O}$ traço e a Densidade a posteriori de $\beta_{1}, \beta_{2}, \xi_{1}, \xi_{2}, \gamma_{1}$ e $\gamma_{2}$.

Tabela 4.6: Resultados obtidos para o Critério DIC.

\begin{tabular}{c|c|c|c}
\hline Modelo & No $^{\circ}$ bs. & DIC & N. $^{\circ}$ Paràmetros \\
\hline \hline M1 & 20 & 4000170 & 5 \\
\hline M2 & 20 & 4000170 & 5 \\
\hline M 3 & 20 & 4000160 & 7 \\
\hline M4 & 20 & 4000160 & 6 \\
\hline \hline
\end{tabular}




\section{Capítulo 5}

\section{Uma Abordagem Bayesiana para um Ponto de Mudança em PPNH}

Em muitas aplicações, temos a presença de um ponto de mudança na função intensidade de processos PPNH. Nesta situação, temos interesse em obter a inferência deste ponto de mudança onde o processo de Poisson não-homogêneo muda. Nossa contribuição neste capítulo e estimar os parâmetros dos modelos de taxa de risco com ponto de mudança. Devido a irregularidade dos modelos, o método clássico do estimador de máxima verossimilhança e método dos momentos não podem ser usados. Assim, vamos considerar o uso do método MCMC para desenvolver uma análise Baysiana para ponto de mudança considerando processos de lei de potência e processo de Musa Okumoto no caso discreto e contínuo para estimar os parâmetro de interesse.

\subsection{Ponto de Mudança nos Processos de Lei de Potência}

Considere a superposição do processo PPNH de lei de potência (PLP) introduzido no Capítulo 4 com a taxa de ocorrência ou função de intensidade dada em (4.1).

Considerando o caso especial $3 \neq 1$ temos um processo de Poisson não Homogèneo e assumimos que existe um ponto de mudança $\tau$ no período de observação $(0, T]$ durante o qual $N(s)=n$ eventos ocorrem no tempo 0 a s. Observe que estamos assumindo que o tempo entre as falhas para um processo de Poisson não-homogèneo segue um processo de lei de potência. Denotamos $\tau(1 \leq \tau \leq n)$ o ponto de mudlança de um processo de lei de potência. Salientamos que na estimação do ponto de mudança, assumimos que os tempos de vida não são identicamente independentes. 
Vamos assumir que existe um ponto de mudança $\tau$, tal que,

$$
\lambda(t)= \begin{cases}\lambda_{1}(t), & \text { se } 0 \leq t \leq \tau \\ \lambda_{2}(t), & \text { se } t>\tau\end{cases}
$$

onde $\lambda_{j}(t)$ é dado (4.1) no capítulo 4 .

Seja $\mathfrak{D}_{\mathrm{t}}$ o conjunto de dados observados até o tempo $T$, que consiste em $n$ épocas ordenadas $\left(t_{1}, t_{2}, \ldots, t_{n}\right)$, onde $0<t_{1}<t_{2}<\cdots<t_{n}<T$. Assumindo que o processo pontual de épocas ordenadas segue o PPNH com função de intensidade dada em (4.1) e um ponto de mudança $\tau$, a função de verossimilhança de $\underline{\nu}=\left(\theta_{1}, \theta_{2}, \beta_{1}, \beta_{2}, \tau\right)$ considerando o modelo de tempo truncado é dada por:

$$
L(\underline{\nu})=\prod_{i=1}^{M(\tau)} \lambda_{1}\left(t_{i}\right) e^{-m\left(t_{M(\tau)}\right)} \times \prod_{i=M(\tau)+1}^{M(T)} \lambda_{2}\left(t_{i}\right) e^{-m\left(t_{M(T)}\right)}
$$

onde $M(t)$ é o número de eventos ocorridos entre intervalo $(0, t]$.

Para uma análise Bayesiana do modelo, vamos assumir a seguintes distribuições a priori para os parametros

$$
\begin{aligned}
& \theta_{j} \sim \Gamma\left[a_{j}, b_{j}\right] \\
& \beta_{j} \sim \Gamma\left[c_{j}, d_{j}\right]
\end{aligned}
$$

para $j=1,2 ; a_{j}, b_{j}, c_{j}$ e $d_{j}$ os hiperparâmetros são conhecidos e uma distribuição a priori não-informativa para $\tau ; \Gamma[a, b]$ denota uma distribuição gama com média $a / b$ e variância $a / b^{2}$. Também assumimos independência a priori entre os parâmetros $\theta_{j}, \beta_{j}(j=1,2)$.

A distribuição a posteriori conjunta para $\underline{\nu}$ assumindo uma distribuição a priori discreta uniforme para $\tau$, em cada valor observado $t_{i}$, isto é, $\pi\left(\tau=t_{i}\right)=1 / n, i=1, \ldots, n$ é dada por:

$$
\begin{aligned}
\pi\left(\underline{\nu} \mid \mathfrak{D}_{\mathrm{t}}\right) & \times \theta_{1}^{a_{1}-1} e^{-b_{1} \theta_{1}} \theta_{2}^{a_{2}-1} e^{-b_{2} \theta_{2}} \beta_{1}^{c_{1}-1} e^{-d_{1} \beta_{1}} 3_{2}^{c_{2}-1} e^{-d_{2}, \beta_{2}} \times\left(\frac{\beta_{1}}{\theta_{1}}\right)^{M(\tau)}\left[\prod_{i=1}^{M(\tau)}\left(\frac{t_{i}}{\theta_{1}}\right)^{\beta_{1}-1}\right] \\
& \times \exp \left\{-\left(\frac{t_{M(\tau)}}{\theta_{1}}\right)^{3_{1}}\right\}\left(\frac{3_{2}}{\theta_{2}}\right)^{M(T)-M(\tau)}\left[\prod_{i=M(\tau)-1}^{M(T)}\left(\frac{t_{i}}{\theta_{2}}\right)^{3_{2}-1}\right] \exp \left\{-\left(\frac{t_{M(T)}}{\theta_{2}}\right)^{\beta_{2}}\right\},(5 .)
\end{aligned}
$$

onde $\theta_{1}, \theta_{2}, 3_{1}, 3_{2}>0$ e $\tau=t_{1} \cdot t_{2}, \ldots t_{n}$.

Também podemos assumir a distribuição a priori não-informativa contínua para $\tau$. Uma possível escolha é dada por $\pi(\tau) \propto \frac{1}{\tau}$ para $\tau>0$. 
As densidades a posteriori condicional necessárias para o algoritmo Gibbs Sampling são dadas por:

(i) $\pi\left(\theta_{1} \mid \underline{\nu}_{\left(\theta_{1}\right)}, \mathfrak{D}_{\mathfrak{t}}\right) \propto \theta_{1}^{a_{1}-1} e^{-b_{1} \theta_{1}} \psi_{1}(\underline{\nu})$,

onde:

$$
\psi_{1}(\underline{\nu})=\exp \left\{-M(\tau) \ln \theta_{1}-\left(\beta_{1}-1\right) M(\tau) \ln \theta_{1}-\left(\frac{t_{M(\tau)}}{\theta_{1}}\right)^{\beta_{1}}\right\}
$$

(ii) $\pi\left(\theta_{2} \mid \underline{\nu}_{\left(\theta_{2}\right)}, \mathfrak{D}_{\mathrm{t}}\right) \propto \theta_{2}^{a_{2}-1} e^{-b_{2} \theta_{2}} \psi_{1}(\underline{\nu})$,

onde:

$$
\psi_{2}(\underline{\nu})=\exp \left\{-(M(T)-M(\tau)) \ln \theta_{2}-\left(\beta_{2}-1\right)(M(T)-M(\tau)) \ln \theta_{2}-\left(\frac{t_{M(T)}}{\theta_{2}}\right)^{\beta_{2}}\right\}
$$

(iii) $\pi\left(\beta_{1} \mid \underline{\nu}_{\left(\beta_{1}\right)}, \mathfrak{D}_{\mathfrak{t}}\right) \propto \beta_{1}^{c_{1}-1} e^{-d_{1} \beta_{1}} \psi_{3}(\underline{\nu})$,

onde:

$$
\psi_{3}(\underline{\nu})=\exp \left\{M(\tau) \ln \beta_{1}+\beta_{1} \sum_{i=1}^{M(\tau)} \ln \left(\frac{t_{i}}{\theta_{1}}\right)-\left(\frac{t_{M(\tau)}}{\theta_{1}}\right)^{\beta_{1}}\right\}
$$

(iv) $\pi\left(\beta_{2} \mid \underline{\nu}_{\left(\beta_{2}\right)}, \mathfrak{D}_{\mathfrak{t}}\right) \propto \beta_{2}^{c_{2}-1} e^{-d_{2}, \beta_{2}} \psi_{4}(\underline{\nu})$,

onde:

$$
u_{4}(\underline{\nu})=\exp \left\{(M(T)-M(\tau)) \ln 3_{2}+3_{2} \sum_{i=M(\tau)-1}^{M(T)} \ln \left(\frac{t_{i}}{\theta_{2}}\right)-\left(\frac{t_{M(T)}}{\theta_{2}}\right)^{3_{2}}\right\}
$$

(v) $\pi\left(\tau=t_{i} \mid \underline{\nu}_{(\tau)}, \mathfrak{D}_{\mathrm{t}}\right) \times \nu_{5}(\underline{\nu})$, 
onde:

$$
\begin{aligned}
\psi_{5}(\underline{\nu})= & \exp \left\{M(\tau)\left(\ln \beta_{1}+2 \ln \theta_{2}-\ln \theta_{1}\right)+\left(\beta_{1}-1\right) \sum_{i=1}^{M(\tau)} \ln \left(\frac{t_{i}}{\theta_{1}}\right)+\right. \\
& \left.\left(\beta_{2}-1\right) \sum_{i=M(\tau)+1}^{M(T)} \ln \left(\frac{t_{i}}{\theta_{2}}\right)-\left(\frac{t_{M(\tau)}}{\theta_{1}}\right)^{\beta_{1}}\right\}
\end{aligned}
$$

onde $\underline{\nu}_{(j)}=\left(\nu_{1}, \ldots, \nu_{j-1}, \nu_{j+1}, \ldots, \nu_{p}\right)$.

Observe que devemos usar o algoritmo de Metropolis-Hastings para simular as amostras a posteriori dos parâmetos $\theta_{1}, \theta_{2}, \beta_{1}, \beta_{2}$ e $\tau$.

\subsection{Ponto de Mudança do Processo de Musa - Oku- moto}

Nesta seção vamos considerar a superposição do processo de PPNH introduzido por Musa e Okumto(1984). A função de intensidade dada por:

$$
\lambda_{j}(t)=\frac{\beta_{j}}{t+\theta_{j}}
$$

para $j=1,2$.

Associado à função de intensidade (5.6) temos a função de valor médio dada por $m_{j}(t)=\beta_{j} \ln \left(1+\frac{t}{\theta_{j}}\right)$, para $j=1,2$.

Também assumindo um ponto de mudança $\tau$, e a mesma distribuição a priori (5.3) para $\theta_{1}, \theta_{2}, \beta_{1}$, e $\beta_{2}$ e uma distribuição a priori discreta não uniforme para o ponto de mudança $\tau$; a distribuição a posteriori conjunta para $\underline{\nu}=\left(\theta_{1}, \theta_{2}, \beta_{1}, \beta_{2}, \tau\right)$ é dada por:

$$
\begin{aligned}
\pi\left(\underline{\nu} \mid \mathfrak{D}_{\mathbf{t}}\right) & \propto \theta_{1}^{a_{1}-1} e^{-b_{1} \theta_{1}} \theta_{2}^{a_{2}-1} e^{-b_{2} \theta_{2}} \beta_{1}^{c_{1}-1} e^{-d_{1} \beta_{1}} \beta_{2}^{c_{2}-1} e^{-d_{2} \beta_{2}} \beta_{1}^{M(\tau)} \times\left\{\prod_{i=1}^{M(\tau)} \frac{1}{t_{i}+\theta_{1}}\right\} \\
& \times \exp \left\{-\beta_{1} \ln \left(1+\frac{t_{M(t)}}{\theta_{1}}\right)\right\} 3_{1}^{M(T)-M(\tau)} \times\left\{\prod_{i=M(\tau)-1}^{M(T)}\left(\frac{1}{t_{i}+\theta_{1}}\right)\right\} \\
& \times \exp \left\{-\beta_{2} \ln \left(1+\frac{t_{M(T)}}{\theta_{2}}\right)\right\}
\end{aligned}
$$

onde $\theta_{1}, \theta_{2}, 3_{1}, 3_{2}>0$ e $\tau=t_{1}, t_{2}, \ldots, t_{n}$.

As densidades a posteriori condicionais para aplicação do algoritmo Gibbs Sampling são dlaclas por: 
(i) $\beta_{1} \mid \underline{\nu}_{\left(\beta_{1}\right)}, \mathfrak{D}_{\mathfrak{t}} \sim \Gamma\left[c_{1}+M(\tau), d_{1}+\ln \left(1+\frac{t_{M(\tau)}}{\theta_{1}}\right)\right]$;

(ii) $\beta_{2} \mid \underline{\nu}_{\left(\beta_{2}\right)}, \mathfrak{D}_{\mathfrak{t}} \sim \Gamma\left[c_{2}+M(T)-M(\tau), d_{2}+\ln \left(1+\frac{t_{M(T)}}{\theta_{2}}\right]\right.$;

(iii) $\pi\left(\theta_{1} \mid \underline{\nu}_{\left(\theta_{1}\right)}, \mathfrak{D}_{\mathrm{t}}\right) \propto \theta_{1}^{a_{1}-1} e^{-b_{1} \theta_{1}} \psi_{6}(\underline{\nu})$,

onde:

$$
\psi_{6}(\underline{\nu})=\exp \left\{-\sum_{i=1}^{M(\tau)} \ln \left(t_{i}+\theta_{1}\right)-\beta_{1} \ln \left(1+\frac{t_{M(\tau)}}{\theta_{1}}\right)\right\}
$$

(iv) $\pi\left(\theta_{2} \mid \underline{\nu}_{\left(\theta_{2}\right)}, \mathfrak{D}_{\mathrm{t}}\right) \propto \theta_{2}^{a_{2}-1} e^{-b_{2} \theta_{2}} \psi_{7}(\underline{\nu})$,

onde:

$$
\psi_{7}(\underline{\nu})=\exp \left\{\sum_{i=M(\tau)+1}^{M(T)} \ln \left(t_{i}+\theta_{2}\right)-\beta_{2} \ln \left(1+\frac{t_{M(T)}}{\theta_{2}}\right)\right\}
$$

(v) $\pi\left(\tau=t_{i} \mid \underline{\nu}_{(\tau)}, \mathfrak{D}_{\mathrm{t}}\right) \propto \psi_{\mathrm{s}}(\underline{\nu})$,

onde:

$$
\begin{aligned}
\psi_{8}(\underline{\nu})= & \exp \left\{M(\tau) \ln \left(\frac{\beta_{1}}{\beta_{2}}\right)-\sum_{i=1}^{M(\tau)} \ln \left(t_{i}+\theta_{1}\right)-\beta_{1} \ln \left(1+\frac{t_{M(T)}}{\theta_{1}}\right)-\right. \\
& \left.\sum_{i=M(\tau)+1}^{M(T)} \ln \left(t_{i}+\theta_{2}\right)\right\} .
\end{aligned}
$$

Observe que devemos usar o algoritmo de Metropolis-Hastings para simular as amostras a posteriori dos parâmetos $\theta_{1}, \theta_{2}$ e $\tau$.

\subsection{Aplicações Numéricas}

Nesta seção, a metodologia para análise Bayesiana na aplicação númerica é ilustrada com um conjuntos de dados gerados e um conjunto de dados reais. 


\subsubsection{Uma Ilustração Numérica com Dados Simulados a partir do Processo Lei de Potência}

Na Tabela 5.1 apresentamos um conjunto de dados simulados a partir de um processo PPNH com função intensidade dado em (4.1), com os valores $\theta_{1}=2, \theta_{2}=8, \beta_{1}=\beta_{2}=0.5$, e $\tau=10$. Assumimos uma densidade a priori não-informativa discreta e também contínua para $\tau$ e a densidade a priori para $\theta_{1}, \theta_{2}, \beta_{1}$ e $\beta_{2}$ dada em (5.3).

Tabela 5.1: Dados gerados a partir de um processo PPNH com lei de potência.

\begin{tabular}{c|c|c|c|c|c|c}
\hline \hline 0,1235 & 3,8394 & 29,9314 & 50,2999 & 290,9619 & 478,9752 & 1031,86 \\
\hline 0,6658 & 7,8674 & 31,4086 & 207,9458 & 299,0633 & 539,9856 & 1047,1765 \\
\hline 1,2409 & 19,1212 & 49,6908 & 211,2891 & 337,9812 & 640,7769 & \\
\hline \hline
\end{tabular}

Considerando uma densidade a priori discreta para $\tau$ dada por $\pi\left(\tau=t_{i}\right)=1 / n$, $i=1, \ldots, n$ e uma superposição de dois processos de lei de potência, com um ponto de mudança $\tau$, assumimos os seguintes valores para os hiperparâmetros da densidade a priori em (5.3); $a_{1}=200, b_{1}=100, a_{2}=800, b_{2}=100, c_{1}=50, d_{1}=100, c_{2}=100, d_{2}=200$. A escolha desse valores para os hiperparâmetros foi subjetiva. Entretanto, uma pequena análise de sensitividade foi feita escolhendo outros valores para os hiperparâmetros, mas estas escolhas não modificaram substancialmente os resultados apresentados na Tabela 5.2 e resultados correspondentes serão omitidos aqui por simplicidade.

Os sumários a posteriori para os parâmetros de interesse são apresentados na Tabela 5.2. Estes resultados foram obtidos usando o software WinBugs (ver Spiegelhalter et al., 1999) geramos 3 cadeias separada de Gibbs cada uma com 10000 iterações. Para cada cadeia, descartamos os primeiro 4000 elementos (burn-in). Monitoramos a convergência das amostras de Gibbs usando o método de Gelman e Rubin (1992). Para cada parâmetro, consideramos um elemento da amostra em cada dez e assim obtemos aproximadamente uma amostra independente. Também na Tabela 5.2 observamos convergência do algoritmo a partir dos fatores de escala de redução $\hat{R}$ introduzidos por Gelman e Rubin (1992). As estimativas Bayesianas para todos os parâmetros estão próximos dos valores usados para simulação do conjunto dos dados.

Considerando uma densidade a priori uniforme contínua para $\tau$ no intervalo [1, 20], assumimos mesmos valores para os hiperparàmetros da densidlade a priori (5.3), considerado 
Tabela 5.2: Resumos a posteriori (processos lei de potência com um ponto de mudança ( priori discreta)).

\begin{tabular}{c|c|c|c|c}
\hline \hline Parâmetros & Média & D.P & Interv. Credibilidade & $\hat{R}$ \\
\hline \hline$\tau$ & 10,53 & 5,764 & {$[1,00 ; 20,00]$} & 1,030 \\
\hline$\beta_{1}$ & 0,5001 & 0,049 & {$[0,371 ; 0,648]$} & 1,005 \\
\hline$\beta_{2}$ & 0,50 & 0,049 & {$[0,408 ; 0,604]$} & 1,010 \\
\hline$\theta_{1}$ & 1,999 & 0,141 & {$[1,733 ; 2,285]$} & 1,001 \\
\hline$\theta_{2}$ & 8,0 & 0,281 & {$[0,459 ; 8,56)$} & 1,021 \\
\hline \hline
\end{tabular}

acima.

O sumários a posteriori do modelo são apresentados na Tabela 5.3, que foram obtidos usando o software WinBugs onde geramos 3 cadeias Gibbs separadas, cada uma das quais com 10000 iterações. Para cada cadeia, descartamos os primeiros 4000 elementos (burnin). Monitoramos a convergência das amostras de Gibbs usando o método de Gelman e Rubin (1992). Para cada parâmetro, consideramos um elemento da amostra em cada dez e assim obtemos aproximadamente uma amostra independente.

Tabela 5.3: Resumos a posteriori ( processos lei de potencia com um ponto de mudança ( priori contínua)).

\begin{tabular}{c|c|c|c|c}
\hline \hline Parâmetros & Média & D.P & Interv. Credibilidade & $\hat{R}$ \\
\hline \hline$\tau$ & 10,51 & 5,483 & {$[1,489 ; 19,530]$} & 1,001 \\
\hline$\beta_{1}$ & 0,5001 & 0,071 & {$[0,371 ; 0,647]$} & 1,002 \\
\hline$\beta_{2}$ & 0,499 & 0,05 & {$[0,407 ; 0,603]$} & 1,001 \\
\hline$\theta_{1}$ & 2,000 & 0,141 & {$[1,733 ; 2,285]$} & 1,002 \\
\hline$\theta_{2}$ & 8,001 & 0,282 & {$[7,458 ; 8,566)$} & 1,004 \\
\hline \hline
\end{tabular}

Apartir dos resultados da Tabela 5.2 e 5.3 observamos resultados similarares para a inferência dos parâmetros $\beta_{1}, \beta_{2}, \theta_{1}$ e $\theta_{2}$. As médias a posteriori para o ponto de mudança $\tau$ também são muito similares assumindo uma distribuição a priori discreta ou contínua para $\tau$. Apesar disso. observamos uma melhor perfomance do resultado da inferência para o ponto de mudança $\tau$ assumindo uma densidade a priori contínua (menor intervalo de credibilidade). 


\subsubsection{Uma Ilustração Numérica com Conjunto de Dados Reais}

Vamos assumir os dados de acidentes de minas de carvão analisados por vários autores (ver por exemplo, Jarret, 1979; Raftery e Akman, 1986). No nosso caso, consideramos os dados que foram registrados a partir de 1851 até 1962 na Inglaterra dado em dias começando em primeiro de janeiro de 1951 (ver Tabela 5.4). Na figura 5.1 apresentamos o gráfico com os números de acidentes (deste de 1851 até 1962) para cada ano.

Da Figura 5.1, observamos que existe um ponto de mudança para a frequência dos acidentes próximo do ano de 1990.

Para analisar o conjunto de dados da Tabela 5.4, primeiro assumimos o processo de Musa Okumoto com um ponto de mudança $\tau$ com uma densidade a priori discreta para $\tau$ dado por $\pi\left(\tau=t_{i}\right)=1 / n, i=1, \ldots, n$, onde $n=486$ (número de acidentes entre 1851 e 1962) e a densidade a priori gama para $\beta_{1}, \beta_{2}, \theta_{1}$ e $\theta_{2}$ dada em (5.3) com os valores dos hiperparâmetros dados por $a_{1}=50000, b_{1}=10, a_{2}=64500, b_{2}=10, c_{1}=12000$, $d_{1}=10, c_{2}=2460, d_{2}=10$; simulamos 3 cadeias Gibbs separadas, descartamos os primeiros 4000 elementos da cada cadeia. Na Tabela 5.5, temos os sumários a posteriori para cada parâmetro. Na Tabela 5.5, também temos o fator de escala de redução $\hat{R}$. Observamos a convergência para todos os parâmetros. Também foi feita uma pequena análise de sensitividade escolhendo outros valores para os hiperparâmetros, mas estas escolhas não modificaram substancialmente os resultados apresentados na Tabela $5.2 \mathrm{e}$ resultados correspondentes serão omitidos aqui por simplicidade.

É importante salientar que a escolha dos valores dos hiperparâmetros da densidade a priori foi feita por métodos Bayesianos empíricos, comparando o número observado de acidentes acumulados com a fórmula da função do valor médio do processo de Musa e Okumoto e processo Lei de potência.

Assumindo o processo da lei de potência com um ponto de mudança e a mesma distribuição considerada para o processo de Musa e Okumoto com um ponto de mudança e com os valores dos hiperparâmetros para as distribuições a priori dados por $a_{1}=1100$, $b_{1}=100, a_{2}=3500, b_{2}=10000, c_{1}=82 . d_{1}=100, c_{2}=530, d_{2}=1000$ escolhidos empiracamente, simulamos 3 cadeias Gibbs separadlas onde clescartamos os primeiros 4000 elementos. Na Tabela 5.6 temos os sumários a posteriori para todos os parâmetros. 


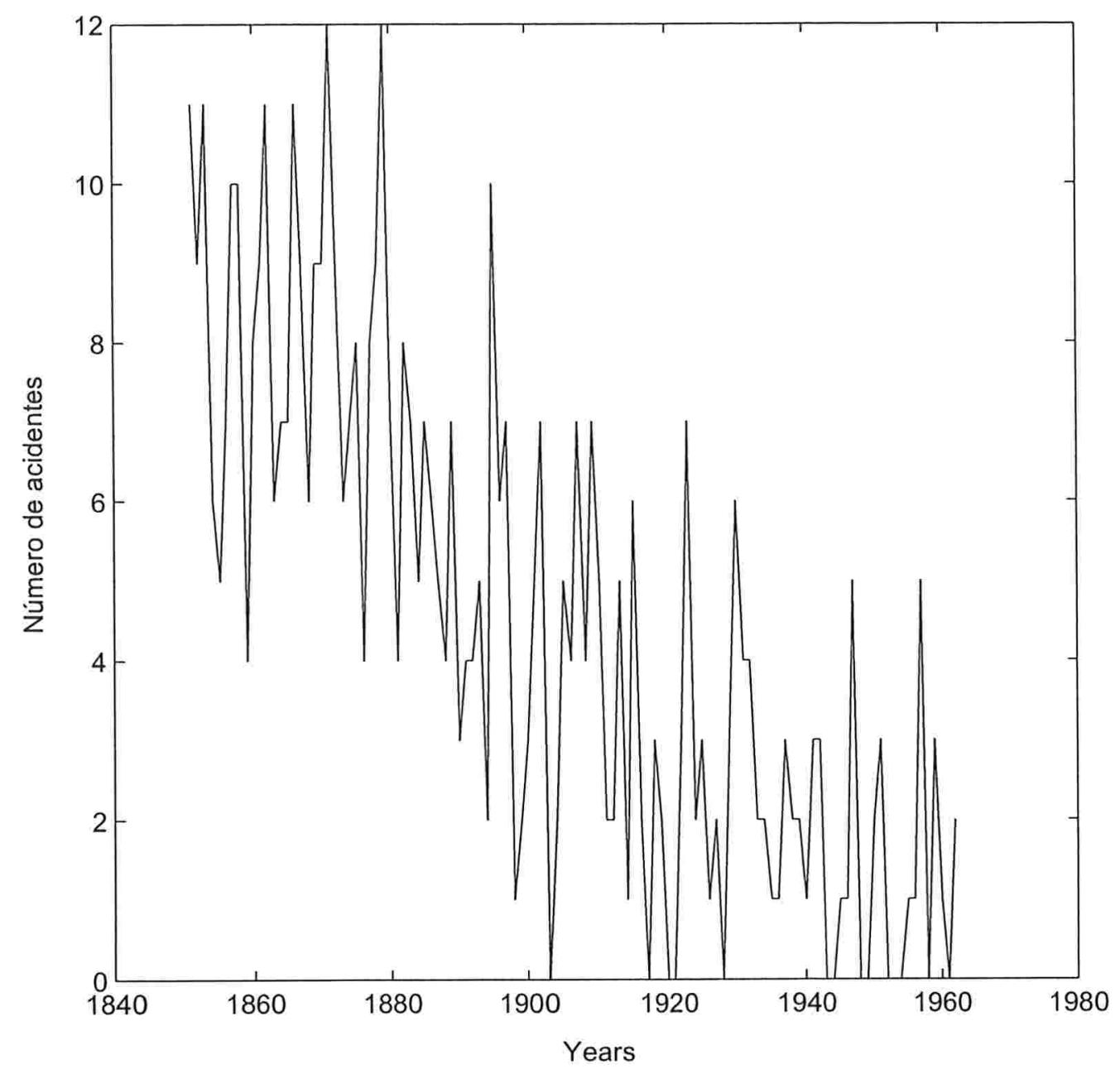

Fiqure 5.1 : Número de acindentes em minas de carvãona Inqlaterra de 1851 a 1962

Apartir dos resultados das Tabelas 5.5 e 5.6, observamos resultados muito próximos para o ponto de mudança assumindo processos de Musa e Okumoto e de lei de potências.

Na Figura 5.2 plotamos empiricamente a função do valor médio e as funções ajustadas de valor médio assumindo o processo de Musa e Okumoto e o processo lei de potência com um ponto de mudança.

A partir da Figura 5.2, observamos que o processo de Mrusa e Okumoto com um ponto de mudança leva a melhor ajuste ao conjunto dos clados cla Tabela 5.4. Observar também que o ponto de mudança $\tau$ tem uma média a posteriori próxima de 243 (ver Tabelas 5.5 e 5.6) assumindo os processos de Musa e Okumoto ou o processo de lei de potência, isto é, isso corresponde ao número de 11321 clias (ou ano de 1882). Este resultado está de acordo e bem próximo com as frequencias observadas dos acidentes em cada ano plotado (ver Figura 5.1). Vamos mostrar a seguir para efeito de comparação alguns métodos utilizados 
na literatura para estimar o intervalo do ponto de mudança para o mesmo conjunto de dados.

\begin{tabular}{|c|c|c|c|}
\hline \hline Método & $\widehat{\tau}$ & Limite esquerda & limite a direita \\
\hline Akman e Raftery (1992) & 10 de Mar, de 1980 & 6 de Out, de 1986 & 17 de Dez, de 1998 \\
Raftery e Akman (1986) & 27 de Aug, de 1980 & 15 Mar, de 1987 & 2 de Aug, de 1895 \\
Woraley (1986) & 1890 & 1884 & 1895 \\
\hline \hline
\end{tabular}

Para a seleção do modelo podemos usar um dos métodos Bayesianos introduzidos na seção 1.4 do capítulo 1. Neste caso, considerando o fator de Bayes o qual é o peso relativo de evidência para modelo $M_{1}$ (ponto de mudança em processo de Musa-Okumoto) comparado com o modelo $M_{2}$ (ponto de mudança em processo lei de potência) o fator de Bayes mostra que o modelo $M_{1}$ é o preferível sob o modelo $M_{2}$ desde que $B_{12}=$ $2,96 \times 10^{113}>1$.

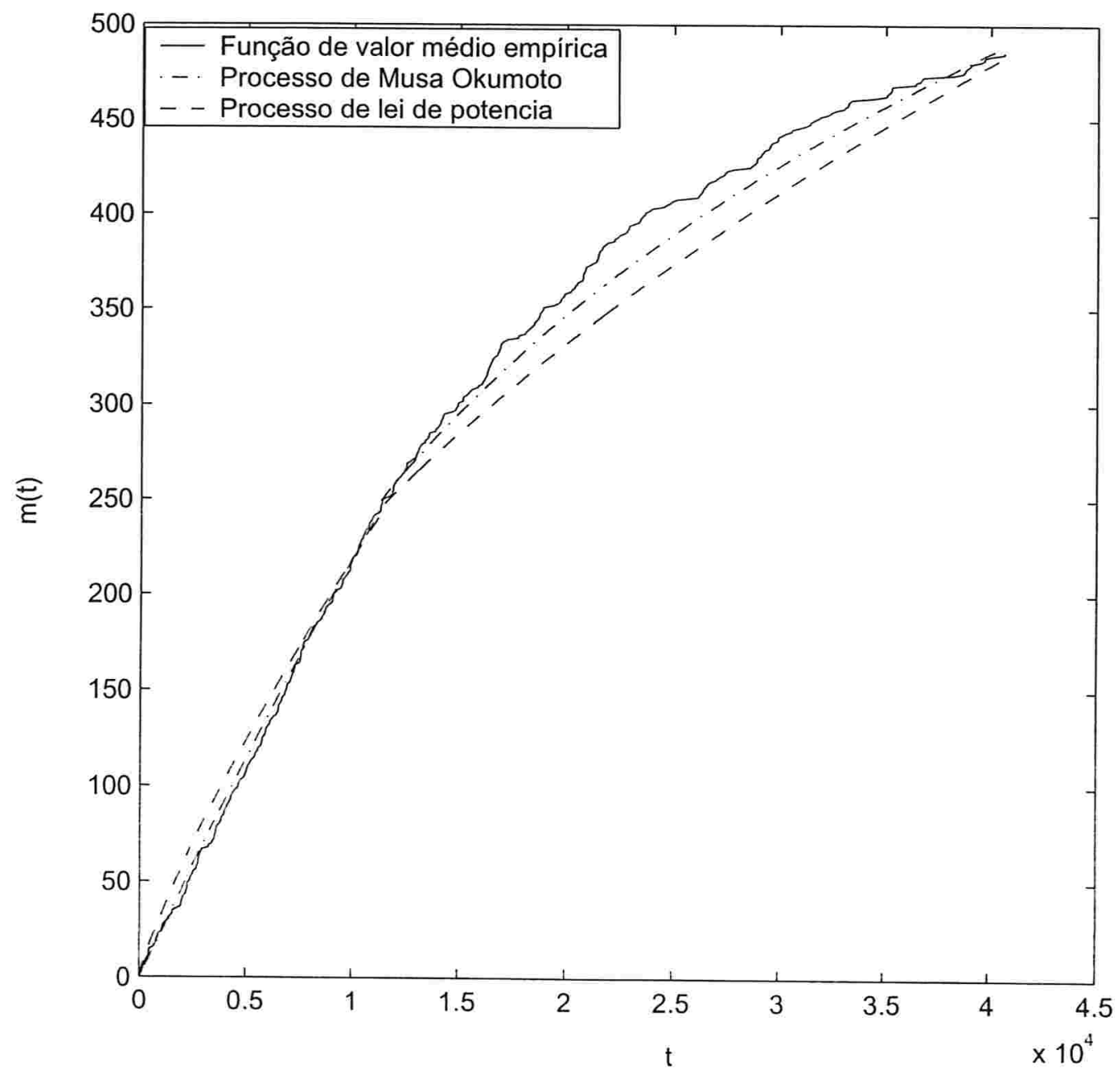

Figura 5.2: Gráficos para as funções de valor médio. 
Tabela 5.4: Conjunto dos Número de dias acumulados de acidentes em uma mina de carvão na Inglaterra no período de 1851 a 1962.

\begin{tabular}{|c|c|c|c|c|c|c|c|c|c|}
\hline 2 & 2330 & 4591 & 6808 & $\overline{9055}$ & 11321 & 14265 & 18573 & 22904 & 30058 \\
\hline 84 & 2371 & 4635 & 6812 & 9057 & 11332 & 14653 & 18663 & 22908 & 30245 \\
\hline 117 & 2401 & 4644 & 6866 & 9060 & 11382 & 14804 & 18692 & 23132 & 30494 \\
\hline 147 & 2437 & 4677 & 6895 & 9087 & 11383 & 14834 & 18754 & 23364 & 30552 \\
\hline 157 & 2506 & 4728 & 6903 & 9179 & 11395 & 14903 & 17755 & 23403 & 30907 \\
\hline 173 & 2528 & 4849 & 6925 & 9260 & 11585 & 14936 & 18864 & 23463 & 31236 \\
\hline 230 & 2621 & 4919 & 7050 & 9435 & 11815 & 14940 & 18881 & 23528 & 31404 \\
\hline 280 & 2695 & 4937 & 7066 & 9471 & 11866 & 15143 & 18915 & 23611 & 31429 \\
\hline 282 & 2704 & 4987 & 7093 & 9486 & 11872 & 15163 & 19384 & 23642 & 31566 \\
\hline 340 & 2746 & 5065 & 7132 & 9513 & 11914 & 15165 & 19556 & 23829 & 31778 \\
\hline 406 & 2758 & 5036 & 7143 & 9517 & 11930 & 15315 & 19716 & 23938 & 31878 \\
\hline 418 & 2779 & 5072 & 7230 & 9549 & 11933 & 15393 & 19717 & 24455 & 32271 \\
\hline 422 & 2781 & 5092 & 7237 & 9626 & 11950 & 15477 & 19764 & 24633 & 32414 \\
\hline 432 & 2817 & 5155 & 7249 & 9732 & 12020 & 15555 & 19887 & 24788 & 32559 \\
\hline 459 & 2841 & 5199 & 7282 & 9743 & 12031 & 15831 & 19892 & 24904 & 32998 \\
\hline 648 & 2866 & 5287 & 7288 & 9861 & 12149 & 15871 & 20178 & 25101 & 33054 \\
\hline 678 & 2916 & 5386 & 7476 & 9877 & 12254 & 16036 & 20212 & 26098 & 33203 \\
\hline 728 & 2933 & 5409 & 7504 & 9880 & 12317 & 16059 & 20347 & 26152 & 33210 \\
\hline 740 & 3248 & 5420 & 7521 & 9884 & 12384 & 16138 & 20374 & 26235 & 33257 \\
\hline 773 & 3264 & 5521 & 7546 & 9899 & 12431 & 16173 & 20472 & 26322 & 33386 \\
\hline 808 & 3401 & 5529 & 7553 & 9963 & 12468 & 16236 & 20489 & 26324 & 34368 \\
\hline 839 & 3418 & 5562 & 7554 & 9971 & 12524 & 16274 & 20702 & 26385 & 35013 \\
\hline 852 & 3499 & 5691 & 7562 & 9988 & 12539 & 16296 & 20739 & 26478 & 35045 \\
\hline 991 & 3513 & 5702 & 7633 & 10067 & 12541 & 16318 & 20744 & 26537 & 35162 \\
\hline 1004 & 3538 & 5735 & 7644 & 10191 & 12727 & 16359 & 20755 & 26606 & 35268 \\
\hline 1014 & 3557 & 5741 & 7655 & 10203 & 12785 & 16389 & 20773 & 26881 & 35269 \\
\hline 1043 & 3577 & 5744 & 7688 & 10241 & 12960 & 16436 & 20841 & 26974 & 35287 \\
\hline 1071 & 3605 & 5823 & 7709 & 10244 & 12988 & 16460 & 20854 & 27084 & 36400 \\
\hline 1195 & 3626 & 5864 & 7902 & 10270 & 13010 & 16513 & 20890 & 27308 & 36419 \\
\hline 1314 & 3635 & 5912 & 7940 & 10275 & 13071 & 16530 & 1021 & 27352 & 36664 \\
\hline 1337 & 3730 & 5913 & 7962 & 10353 & 13078 & 16626 & 21203 & 27439 & 36702 \\
\hline 1398 & 3787 & 5917 & 7977 & 10361 & 13110 & 16753 & 21371. & 27790 & 36789 \\
\hline 1497 & 3820 & 5994 & 8036 & 10433 & 13149 & 16780 & 21395 & 28538 & 38225 \\
\hline 1517 & 3837 & 6074 & 8082 & 10473 & 13248 & 16840 & 21430 & 28652 & 38668 \\
\hline 1532 & 3873 & 6078 & 8101 & 10494 & 13397 & 16860 & 21458 & 28793 & 38759 \\
\hline 1719 & 3950 & 6123 & 8140 & 10536 & 13407 & 16900 & 21461 & 28881 & 38772 \\
\hline 1897 & 3976 & 6198 & 8312 & 10564 & 13512 & 16904 & 21500 & 28883 & 38884 \\
\hline 1924 & 3983 & 6263 & 8332 & 10613 & 13562 & 16936 & 21540 & 28895 & 39030 \\
\hline 1937 & 4033 & 6401 & 8456 & 10732 & 13575 & 17026 & 21648 & 29060 & 39055 \\
\hline 1949 & 4083 & 6457 & 8551 & 10740 & 13600 & 17248 & 21652 & 29100 & 39549 \\
\hline 1978 & 4117 & 6483 & 8556 & 10759 & 13880 & 17731 & 21777 & 29172 & 39698 \\
\hline 2026 & 4183 & 6492 & 8621 & 10796 & 13903 & 17734 & 21876 & 29227 & 39720 \\
\hline 2070 & 4241 & 6.509 & 8676 & 10888 & 14010 & 18050 & 22215 & 29437 & 39982 \\
\hline 2168 & 4275 & 6522 & 8693 & 10928 & 14079 & 18099 & 22234 & 29495 & 40614 \\
\hline 2181 & 4293 & 6584 & 8710 & 10947 & 14120 & 18167 & 22390 & 29515 & 40635 \\
\hline 2216 & 4296 & 6638 & 8722 & 11032 & 14139 & 18281 & $22+42$ & 29581 & \\
\hline 2234 & 4366 & 54 & 8837 & 11262 & 1.4185 & 18379 & 22655 & 29840 & \\
\hline 2265 & 4409 & & 8971 & 11316 & 14218 & 18.473 & 22784 & 29873 & \\
\hline 2200 & 1478 & & 9041 & 11318 & 14233 & 18488 & 22832 & 29877 & \\
\hline
\end{tabular}


Tabela 5.5: Resumos a posteriori (processos de Musa - Okumoto).

\begin{tabular}{c|c|c|c|c}
\hline \hline Parâmetros & Média & D.P & Interv. de Credibilidade & $\hat{R}$ \\
\hline \hline$\tau$ & 243,6 & 140,5 & {$[13,0 ; 474]$} & 1,001 \\
\hline$\beta_{1}$ & 1200,0 & 10,93 & {$[1179,0 ; 1222,0]$} & 1,001 \\
\hline$\beta_{2}$ & 246,0 & 4,951 & {$[236,4 ; 255,8]$} & 1,004 \\
\hline$\theta_{1}$ & 50000 & 70,72 & {$[49860 ; 50140]$} & 1,000 \\
\hline$\theta_{2}$ & 6450,0 & 25,41 & {$[6400,0 ; 6500,0]$} & 1,006 \\
\hline \hline
\end{tabular}

Tabela 5.6: Sumários a posteriori (processos lei de potência).

\begin{tabular}{c|c|c|c|c}
\hline \hline Parâmetros & Média & DP & Interv. de Credibilidade & $\hat{R}$ \\
\hline \hline$\tau$ & 243,3 & 140,5 & {$[13,0 ; 474,0]$} & 1,001 \\
\hline$\beta_{1}$ & 0,819 & 0,091 & {$[0,653 ; 1,005]$} & 1,001 \\
\hline $3_{2}$ & 0,529 & 0,023 & $0,486 ; 0,576]$ & 1,004 \\
\hline$\theta_{1}$ & 14,0 & 0,374 & $13,27 ; 14,74]$ & 1,002 \\
\hline$\theta_{2}$ & 0.35 & 0.006 & {$[0.338: 0.362]$} & 1.008 \\
\hline \hline
\end{tabular}




\section{Capítulo 6}

\section{Conclusões e Pesquisas Futuras}

Nesta tese, utilizamos os métodos Bayesianos de simulação de Monte Carlo via Cadeias de Markov(MCMC) para obter os sumários a posteriori dos parâmetros de modelos envolvendo processos de Poisson não-homogêno.

As principais contribuições foram as apresentações dos modelos de superposição dos processos de Poisson não-homogênos: modelo de Musa Okumoto, modelo de uma função de intensidade polinomial, modelo de lei de potência na presença ou não de covariáveis. A outra, é enfoque Bayesiano para um ponto de mundaça no processo PPNH.

De forma geral, pretendemos continuar o estudo de superposição de processo de Poisson não-homogêneo com mais de um ponto de mudança nos processos de lei de potência. 


\section{Apêndice A}

\section{Diagnósticos de Convergência}

Avaliamos a convergência das cadeias geradas utilizando métodos MCMC. Os quatro critérios que apresentamos a seguir estão implementados na biblioteca CODA. Para maiores detalhes ver exemplo Brooks \& Roberts(1995), Gamerman(1996) e Best et al.(1996).

\section{A.1 Diagnóstico de Geweke}

O diagnóstico proposto por Geweke(1992), é baseado em métodos de séries temporais. Este foi desenvolvido para indicar convergência da média a posteriori de um função da quantidade amostrada, $g(\theta)$, em uma única cadeia, isto é, os valores $g\left(\theta^{(j)}\right)$ são calculados a cada iteração da simulação, formando um série temporal. Estima-se a partir da cadeia a variância assintótica $S(0)$ da média de $g(\theta)$.

Resumidamente, o diagnóstico é dado por: divida a cadeia gerada de tamanho $n$ em duas sequencias, onde a primeira possui as $n_{a}$ primeiras iterações e as segunda, as $n_{b}$ últimas iterações, e calcule as médias $m_{a}$ e $m_{b}$ e variâncias assintóticas $\hat{S_{a}}(0)$ e $\hat{S_{b}} \hat{(0)}$, respectivamene, sendo que a variância é determinada pela estimação da densidade espectral. Se a cadeia completa for estacionária, as médias destas sequencias estarão próximas. Então, para as razões $\frac{n_{a}}{n}$ e $\frac{n_{b}}{n}$ fixas, onde $\frac{n_{a}+n_{b}}{n}<1$.

Assim, quando $n \rightarrow \infty$,

$$
\frac{m_{a}-m_{b}}{\sqrt{\frac{S_{a}(0)}{n_{a}}-\frac{S_{b}(0)}{n_{b}}}}
$$

se a sequência gerada for estacionária. Geweke sugere que a aplicação deste teste seja feita descartandfo algumas iterações iniciais e aincla que $n_{a}=0.1 n$ e $n_{b}=0.5 n$. Resultados melhores podem ser obtidos quando a distribuição a posteriori marginal dos paràmetros for aproximadamente normal. Este teste pode ser generalizado para cadeias múltiplas. 


\section{A.2 Diagnóstico de Gelman \& Rubin}

Este método proposto por Gelman \& Rubin (1992) utiliza cadeias múltiplas, inicializadas em pontos distintos, comparando a variância amostral dentre e entre as cadeias, para cada uma das quantidades de interesse $g(\theta)$. Esta comparação é usada para estimar o fator pelo qual o parâmetro de escala da distribuição a posteriori marginal pode ser reduzido, quando o número de iterações é grande. Para obtenção de um resultado me-lhor no teste, as quantidades de interesse devem ter distribuição aproximadamente normal.

Em linhas gerais, gere $B$ cadeias com $2 N$ iteraçãoes. O teste calcula um fator (shrink factor) que mede o decréscimo que seria observado no parâmetro de escala, se mais iterações fossem realizadas. Aproximando para a distribuição $t$-Student, o fator é dado por

$$
\sqrt{\hat{R_{T}}}=\sqrt{\left(\frac{N-1}{N}+\left(\frac{\sigma_{1}}{\sigma_{2}}\right) \frac{B+1}{B}\right)\left(\frac{\nu}{\nu-2}\right)}
$$

onde $\sigma_{1}$ é a variância entre as $B$ médias das cadeias geradas, $\sigma_{2}$ é a média das variâncias das $B$ cadeias, e $\nu$ é o grau de liberdade da distribuição $t-$ Student. Quando $\nu \rightarrow \infty$, o fato $\rightarrow 1$ e a variância dentro das cadeias domina a variância entre as cadeias. Portanto as cadeias deixam de serem influenciadas por seus respectivos valores iniciais. O teste mostra os valores calculados da mediana e do quantil $97,5 \%$ para o fator para cada $g(\theta)$, considerando as $n$ últimas iterações. Quando os dois quantis calculados se aproximam de 1 , as segundas metades das cadeias convergem para a distribuição de equilibrio.

\section{A.3 Diagnóstico de Raftery \& Lewis}

Este método proposto por Raftery \& Lewis (1992) se aplica a uma única cadeia. Este tem por objetivo detectar convergência e fornecer limites para a precisão dos quantis estimados para as funções das quantidades de interesse. Fornece também o número de iterações iniciais a serem descartadas como um burn-in, o tamanho da cadeia a ser gerada considerando uma determinada precisão na estimativa, o espaçamento entre as iterações a serem consideradas e uma medicla, clenotada por $I$, que é clada pela razão entre o número total de iterações e o número mínimo exigido para a precisão clesejada. Para valores de $I$ maior que 1, existe indicação de alta correlação dentre da amostra gerada e provalmente uma falha na convergência, e para I maior do que 5, existe uma indicação de problemas de 
convergência da cadeia, onde por exemplo, reparametrizações ou diferentes valores inciais podem resolver o problema da convergência.

\section{A.4 Diagnóstico de Heidelberger \& Welch}

Heidelgerger \& Welch (1983) propuseram um diagnónstico para detectar um estado transiente inicial na sequência simulada de eventos discretos, que é apropriado para detectar convergência em cadeias únicas geradas pelo mecanismo MCMC. O método utiliza a estatística Cramer-Von-Mises para testar a hipótese de que os valores amostrados para cada quantidade de interesse, formam um processo estacionário. Este diagnóstico pode ser generalizado para cadeias múltiplas, utilizando-se a estatística de Kolmogorov-Smirnov. Segundo Brooks \& Roberts (1995), este método tem baixo pode para detecção de falha de convergência se os tamanho amostral for pequeno.

Além deste diagnóstico, o CODA também apresenta um análise gráfica e estatística resumos, tais como média, desvio-padrão, e alguns quantis para as quantidades de interesse. Em particular, apresenta os gráficos do diagnóstico de Geweke e do diagnóstico de Gelman \& Rubin. 


\section{Apêndice B}

\section{A Distribuição de Poisson Bivariada}

Uma das formas de construir a distribuição de Poisson Bivariada é o chamado Método de Redução Trivariado, (veja Kocherlakota, S e Kocherlakota, K., 1992). Sejam $W_{1}, W_{2}$ e $W_{3}$ três v.a.i. com distribuições de Poisson com parâmetros $\lambda_{1}, \lambda_{2}$ e $\lambda_{3}$, respectivamente. Sejam também as variáveis $X$ e $Y$, dadas por:

$$
X=W_{1}+W_{3} \quad \text { e } \quad Y=W_{2}+W_{3}
$$

Então, a F.G.P. (função geradora de probabilidade) de $(X, Y)$ é dada da seguinte forma:

$$
\phi_{(X, Y)}\left(t_{1}, t_{2}\right)=E\left[t_{1}^{X}, t_{2}^{Y}\right]=E\left[t_{1}^{W_{1}} t_{2}^{W_{2}}\left(t_{1} t_{2}\right)^{W_{3}}\right]
$$

ou seja,

$$
\phi_{(X, Y)}\left(t_{1}, t_{2}\right)=\exp \left[\lambda_{1}^{*}\left(t_{1}-1\right)+\lambda_{2}^{*}\left(t_{2}-1\right)+\lambda_{3}\left(t_{1}-1\right)\left(t_{2}-1\right)\right]
$$

onde, $\lambda_{1}^{*}=\lambda_{1}+\lambda_{3}$ e $\lambda_{2}^{*}=\lambda_{2}+\lambda_{3}$.

Também temos que,

$$
\begin{array}{r}
\operatorname{Cov}(X, Y)=\operatorname{Cov}\left(W_{1}+W_{3}, W_{2}+W_{3}\right)=\operatorname{Var}\left(W_{3}\right)=\lambda_{3} \\
\operatorname{Var}(X)=\operatorname{Var}\left(W_{1}+W_{3}\right)=\lambda_{1}+\lambda_{3} \\
\operatorname{Var}(Y)=\operatorname{Var}\left(W_{2}+W_{3}\right)=\lambda_{2}+\lambda_{3} \\
\rho(X, Y)=\frac{\lambda_{3}}{\left[\left(\lambda_{1}+\lambda_{3}\right)\left(\lambda_{2}+\lambda_{3}\right)\right]^{1 / 2}}
\end{array}
$$

Assim, neste modelo $\rho(X . Y) \geq 0$ pois $\lambda_{3} \geq 0$. 


\section{Apêndice C}

\section{Alguns programas}

Neste apêndice, apresentamos alguns programas computacionais desenvolvido no software Winbugs, utilizado para obter amostras da distribuição de interesse atráves dos algoritmos Gibbs Sampling e Metropolis-Hastings.

1. O programa 1 é referente ao exemplo de aplicação do Modelo M3 introduzido no capitulo 2

\section{Programa 1}

model \{

$$
\begin{aligned}
& c<-10000 \\
& \text { tn }<-791.1197 \\
& x n<-2 \\
& z 3 n<-(\text { beta } / 2) \text { pow }(\text { tn }, 2) \exp (-\operatorname{eta} 2 * x n) \\
& z 4 n<- \text { alpha1exp }(- \text { eta } 1 x n) \log (1+\text { tn/alpha } 2) \\
& \text { for }(i \text { in } 1: N)\{ \\
& z 1[i]<- \text { alpha1exp }(-\operatorname{eta} 1 x[i]) /(t[i]+\text { alpha } 2) \\
& z 2[i]<- \text { beta } t[i] \exp (-\operatorname{et} a 2 x[i]) \\
& z 3[i]<-(\text { beta/2)pow }(t[i], 2) \exp (-\operatorname{eta} 2 x[i]) \\
& z+[i]<-a l p h a 1 \exp (-\operatorname{eta} 1 x[i]) \log (1+t[i] / \text { alpha } 2) \\
& w 1[i]<-z 1[i] \text { pow }(z 3[i] / z 4[i], 1 / 2)+z 2[i] \text { pow }(z 4[i] / z 3[i], 1 / 2) \\
& u 2[i]<-\approx 1[i]+\approx 2[i] \\
& L[i]<-\operatorname{pow}(\exp (-(z \ln +z 3 n-\operatorname{rhopow}((z \operatorname{4n} z 3 n), 1 / 2))), 1 / N)(z 1[i]+ \\
& z 2[i])(1-(r h o / 2)(w 1[i] / w 2[i])) \\
& \text { zeros }[i]<-0
\end{aligned}
$$




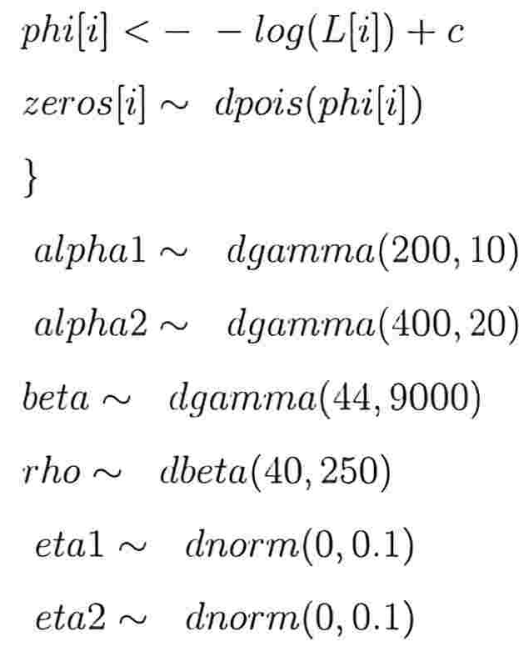

\section{Dados e Condicões Iniciais}

list $(\mathrm{t}=\mathrm{c}(4.0205,6.5164,7.8216,13.4633,14.1814,20.3605,34.6135,35.9622,39.0255,42.3482$, 44.9477,46.9711,58.4492,59.8977,59.9392,63.8705,74.2425,75.0003,79.2846, 94.2169,107.9261,115.1079,127.2589,137.9432,140.1109,141.2993,144.5396, 155.9962,157.4444,195.7686,242.8235,262.3183,268.7236,277.9392,294.4841, $386.1094,386.7154,449.3423,453.4558,505.2807,506.8166,569.1532,608.3752$, 631.3119,638.8890,696.6598,703.7359,728.8963,745.3901,779.5163), $x=c(2,3,3,2,2,3,2,2,2,2,3,2,2,3,2,2,3,2,3,3,3,3,3,3,2,2,3,2,3,2,2,3,3,2,3,3$, $3,2,2,2,3,2,3,2,2,2,3,3,2,2), \mathbf{N}=50$ )

list $($ alpha $1=65$, alpha $2=85$, beta $=0.00021$, eta $1=0.20$, eta $2=65$, rho $=0.397)$ list $($ alpha $1=85$, alpha $2=105$, beta $=0.0003$, eta $1=0.30$, eta $2=60$, rho $=0.3239$ ) list (alpha1 $=95$, alpha2 $=95$, beta $=0.0029$, eta $1=0.25$,eta $2=70$, rho0 .30 ) 
2. O programa 2 é referente ao exemplo de aplicação do Modelo M4 ( superposição de processos dependentes na presença de uma covariável) que introzimos no capitulo 3

\section{Programa 2}

model \{

$$
\begin{aligned}
& x n<-1 \\
& \text { tn }<-398.8943 \\
& \text { for }(i \text { in } 1: N)\{ \\
& z 1[i]<-b e t a 0 * \exp (-\operatorname{eta} 0 * x[i]) \\
& z 2[i]<-\operatorname{beta} 1 t[i] \exp (-\operatorname{eta} 1 x[i]) \\
& z 3[i]<-\operatorname{beta} 0 t[i] \exp (-\operatorname{eta} 0 x[i]) \\
& z 4[i]<-(\text { beta1/2) pow }(t[i], 2) \exp (- \text { eta } 1 x[i]) \\
& w 1[i]<-z 1[i] p o w(z 3[i] / z 4[i], 1 / 2)+z 2[i] \operatorname{pow}(z 4[i] / z 3[i], 1 / 2) \\
& w 2[i]<-z 1[i]+z 2[i] \\
& t[i]<-\operatorname{dpois}(\text { lambda }[i]) \\
& \text { lambda }[i]<-(\text { beta } 0+\text { beta } 1 * t[i]) *(1-((\text { gama } 0+\text { gama } 1) / 2) * \\
& (w 1[i] / w 2[i])) \\
& \text { \} } \\
& \text { beta0 } \sim \operatorname{dgamma}(400 ; 200) \\
& \text { beta } 1 \text { dgamma }(0,001 ; 0.001) \\
& \text { eta } 0 \sim \operatorname{dnorm}(5 ; 10) \\
& \text { eta } 1 \sim \operatorname{dnorm}(3,10) \\
& \text { gama0 } \sim \operatorname{dbeta}(30,70) \\
& \text { gama1 } \operatorname{dbeta}(35,65)
\end{aligned}
$$

\section{Dados e Condicões Iniciais}

$\operatorname{list}(\mathrm{t}=\mathrm{c}(10.6306 .11 .2891,15.6073,20.9628,25.1134,25.1954 .38 .8020,56.1137,74.9975$,

\$5.5960.86.1097.96.2841,96.3057.102.7717.137.3521.148.1138,149.3906.

157.9336.159.3679,159.5329.165.4108,165.9430,173.0731,175.6523,199.1072, 203.0930,

216.6014, 220.7397.228.7703, 232.1089,235.6580,246.0373,219.2204, 252.8160,

255.2921, $271.1487 .290 .5067 .305 .3189,338.0622,3+3.3429,343.3536,349.9357$.

359.3489.362.0208.363.7467,370.1592,381.4956,382.5202,390.5408, 398.89.43),

$x=c(1,1,2.1 .2,1,1.2 .2,2,2,2,2,1,2,1,1.1 .1,1,1,1,1,2.2 .1 .1,1,1,2,2,1,2,2,2,1$, 


$$
2,1,1,1,1,1,1,1,1,2,1,1,1,1), \mathrm{N}=50 \text { ) }
$$

list $($ beta $0=2$, beta $1=3$, eta $0=0.10$, eta $1=0.5$, gama $0=0.25$, gama $1=0.30$ )

$\operatorname{list}($ beta $0=1$, beta $1=1$, eta $0=0.30$, eta $1=0.2$,gama $0=0.35$, gama $1=0.35)$

$\operatorname{list}($ beta $0=1.5$, beta $1=2$, eta $0=5$, eta $1=3$, gama $0=0.30$, gama $1=0.25)$ 
3. O programa 3 é referente ao exemplo de aplicação do Modelo M1 de uma superposição de Poisson não-homogeneos lei de potencias que introzimos no capitulo 4.

\section{Programa 3}

model \{

$$
\begin{aligned}
& c<-100000 \\
& \text { tn }<-047.1765 \\
& z 3 n<-\operatorname{pow}(\text { tn } / \text { teta } 1, \text { beta } 1) \\
& z 4 n<-\operatorname{pow}(\operatorname{tn} / \text { teta } 2, \text { beta } 2) \\
& \text { for }(i \text { in } 1: N)\{ \\
& z 1[i]<-(\text { beta } 1 / \text { teta } 1) * \text { pow }(t[i] / \text { teta } 1, \text { beta } 1-1) \\
& z 2[i]<-(\text { beta } 2 / \text { teta } 2) * \operatorname{pow}(t[i] / \text { teta } 2, \text { beta } 2-1) \\
& z 3[i]<-\operatorname{pow}(t[i] / \text { teta } 1, \text { beta } 1) \\
& z 4[i]<-\operatorname{pow}(t[i] / \operatorname{teta} 2, \text { beta } 2) \\
& w 1[i]<-z 1[i] \text { pow }(z 3[i] / z 4[i], 1 / 2)+z 2[i] \text { pow }(z 4[i] / z 3[i], 1 / 2) \\
& w 2[i]<-z 1[i]+z 2[i] \\
& L[i]<-\operatorname{pow}(\exp (-(z 4 n+z 3 n-\operatorname{rho} * \operatorname{pow}((z 4 n * z 3 n), 1 / 2))), 1 / N) * \\
& (z 1[i]+z 2[i]) *(1-((r h o) / 2) *(w 1[i] / w 2[i])) \\
& z \operatorname{eros}[i]<-0 \\
& \operatorname{phi}[i]<--\log (L[i])+c \\
& z \operatorname{eros}[i] \operatorname{dpois}(p h i[i]) \\
& \text { \} } \\
& \text { beta1 dgamma(1300;2600) } \\
& \text { teta } 1 \text { dgamma }(90 ; 45) \\
& \text { teta } 2 \sim \operatorname{dgmma}(120 ; 15) \\
& \text { rho } \sim \operatorname{dunif}(-1,1)
\end{aligned}
$$

\section{Dados e Condicões Iniciais}

$\operatorname{list}(\mathrm{t}=\mathrm{c}(0.1235,0.6658,1.2 .109,3.8394,7.8674,19.1212,29.9314,31.4086$,

49.6908, 50.2999. 207.9458,211.2891, 290.9619, 299.0633, 337.9812, 478.9752,

$539.9856,640.7769,1031.86,1047.1765), \mathrm{N}=20$ )

list $($ beta $1=0.5$, beta $2=0.5$, teta $1=6$, teta $2=15, \mathrm{rho}=0.2$ )

list $($ beta $1=0.7$, beta $2=0.4$, teta $1=5$, tet $a 2=14, \mathrm{rho}=0.4)$ 
list $($ beta $1=0.6$, beta $2=0.35$, teta $1=2.5$, teta $2=12$, rho $=0.5$ )

list $($ beta $1=0.55$, beta $2=0.75$, teta $1=1.5$, teta $2=15$, rho $=0.7$ ) 
4. O programa 4 é referente ao exemplo de aplicação do Modelo ponto de mudunça no caso discreto que introzimos no capitulo 5 .

\section{Programa 4}

model \{

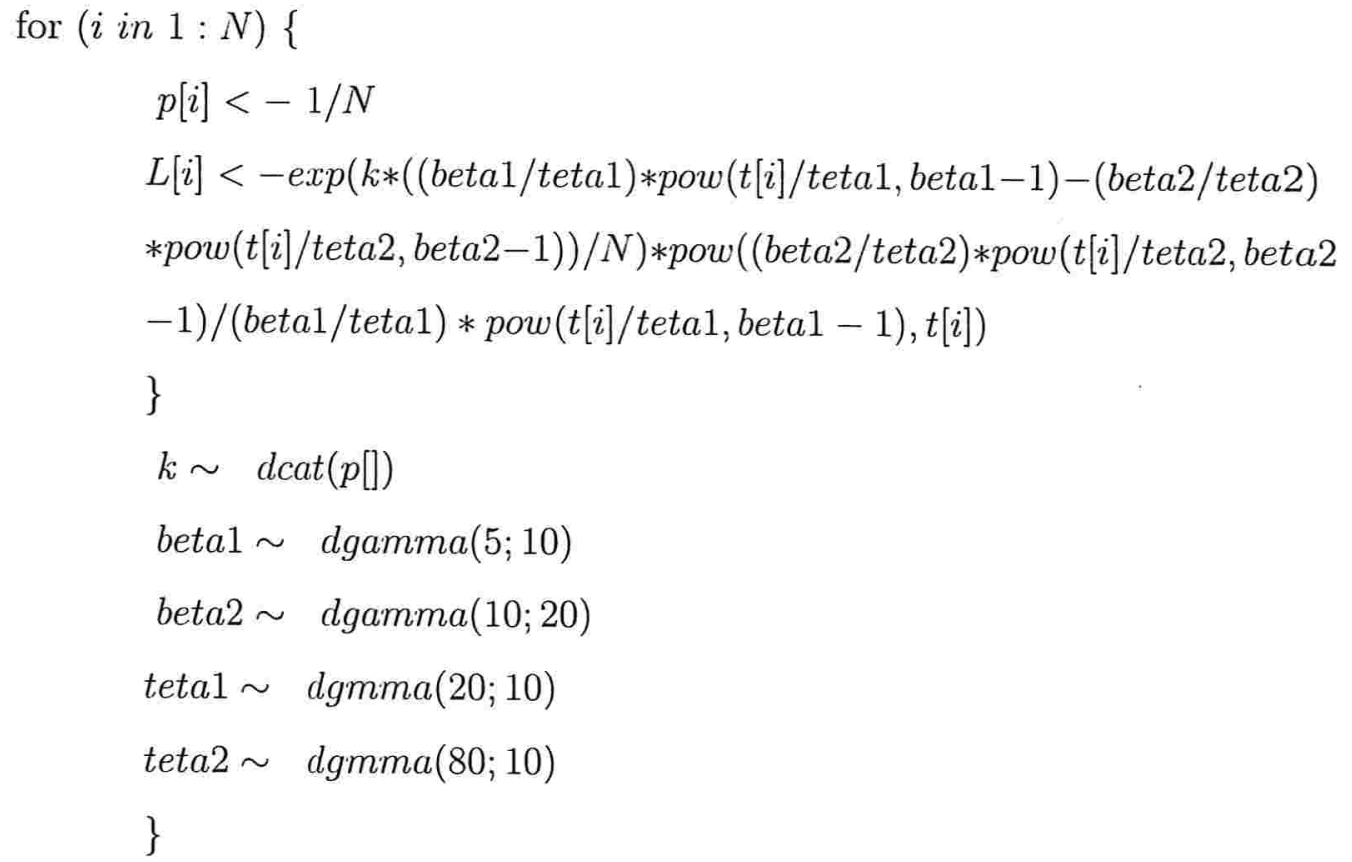

\section{Dados e Condicões Iniciais}

$\operatorname{list}(\mathrm{t}=\mathrm{c}(0.1235,0.6658,1.2409,3.8394,7.8674,19.1212,29.9314,31.4086$, 49.6908, 50.2999, 207.9458,211.2891, 290.9619, 299.0633, 337.9812, 478.9752, $539.9856,640.7769,1031.86,1047.1765), \mathrm{N}=20$ )

$\operatorname{list}(\mathrm{k}=1$, beta $1=0.5$, beta $2=0.5$, teta $1=2$, teta $2=8)$

$\operatorname{list}(\mathrm{k}=2$, beta $1=1.5$, beta $2=1.0$, teta $1=2$, teta $2=8)$

$\operatorname{list}(\mathrm{k}=3$, beta $1=1$, beta $2=0.5$, teta $1=2$, teta $2=8)$ 


\section{Referências Bibliográficas}

[1 ] Aalen, O. O. (1978). Nonparametric inference for a family of counting processes. The Annals of Statistics, 6, 701-726.

[2 ] Achcar,J. A.; Bolfarine, H. (1989). "Constant hazard against a change-point alternative: a Bayesian approach with censored data". Communications in Statistics; Theory and Methods, 18, 10, 3801-3819.

[3 ] Achcar,J. A.; Loibel, S. (1998). "Constant hazard a function models with a changepoint: a Bayesian analysis using Markov Chain Monte Carlo methods". Biometrical Journal, 40, 5, 543-555.

[4 ] Achcar,J. A. (2001). "Bayesian analysis for software reliability data", in : N. Balakrishnan and C. R. Rao (eds), Handbook of Statistics vol 20, pp. 733-748.

[5 ] Achcar,J. A., Dey, D. e Niverthy, M. (1998). A Bayesian approach using nonhomogeneous Poisson process for software reliability models in Frontiers in Reliability . in : Series on Quality, Reliability and Engineering Statistics. vol 4, S.K. Basu and S. Mukhopahyay, eds., Calcuta University India.

[6 ] Andersen, P. K., Borgan, O. ,Gill, R. D. and N.,N. (1993). Statistical models based on counting processes. New York: Spring-Verlag.

[7 ] Aitkin. M. (1991). Posterior Factor J. R. Stat. Soc., B, 53, pp. 111-142.

[8 ] Aslanidou, H., Dey; D. e Sinha, D. (1998) Bayesian Analysis of Multivariate Survival Data using Monte Carlo methods. The Canadian J. of Statistics, La revue Canadienne de Statistique.26,1, pp. 33-18.

9 ] Berger. J. O. and Pericchi. L. R. (1993) The intrinsic Bayes factor. Technical report. Department of statistics, Purdue Lniversity. 
[10 ] Best, N., Cowles, M.K., Vines, K. (1996). CODA (Convergence Diagnosis and Output Analysis for Gibbs sampling output), Manual Version 0.30, MRC Biostatistics Unit, Cambridge, UK.

[11 ] Box, G. E. P. (1980). Sampling and Bayes inference in scientific modeling and Robustness. J. R. Stat. Soc. A, 143, pp. 383-430.

[12 ] Basawa, I. V., and Prakasa Rao, B. L. S. (1980).Statistical Inference for Stochastics Process. London: Academic Press.

[13 ] Campodonico, S., e Singpurwalla, N. D. (1995). Inference and predictions from Poisson point processes incorporating expert knowledge. J. Amer. Statist. Assoc., 90,429 pp. $220-226$.

[14 ] Casella, G. and George, E. I. (1992). Explaining the Gibbs Sampler. Amer. Statistician,46, pp. 167-174.

[15 ] Chib, S. and Greenberg, E. (1995). Understanding the Metropolis-Hasting algorithm. The American Statistician,49, pp.327-335.

[16 ] Cinlar, E. (1975). Introduction to Stochastic Process. Englewood Cliffs, New Jersey, Prentice-Hall.

[17 ] Cox, D. R., and Lewis, P. A. (1966). Statistical Analysis of Series of Events. London. Methuem.

[18 ] Crowder, M. J., Kimber, A. C., Smith, R. L., and Sweeting, T.T. ( (1991). Statistical Analysis of Reliability. London : Chapnab and Hall.

[19 ] Dey, D. K., Sinha. D. (1998). Bayesian model cletermination in lifetime clata analysis.Rebrape, 12, 2, 113-130.

[20 ] Dharmadhikari, A. D.. Naik-Nimbalkar, U. V.. Bhyri, S (1989). Estimation of the scale parameter of a power law process using power using power law counts. Annals of the Intitute of Statistical Mathematics.41. 1, 139-148.

[21 ] Duane. T. T. (1966). IEEE Transactions on Aerospace.AS-2(2), 563-566. 
[22 ] Fleming, T. R., Harrington, D. P. (1991). Counting processes and survival analysis. New York: Willey.

[23 ] Gamerman, D. (1996). Simulação estocástica via Cadeia de Markov. XII Simpósio Nacional de Probabilidade e Estatística, Associação Brasileira de Estatística.

[24 ] Gaver, D. P. and Acar, M. (1979). Analytical hazard representations for use in reliability, mortality and simulation studies. Comm in Statist., Sim. and Com., B8, pp. 91-111.

[25 ] Geisser, S., Eddy, W. (1979). A predictive approach to model selection J. Amer. Statist. Assoc., 74,pp. 153-160.

[26 ] Gelman, S., and Gelman, D. (1984). Stochastics relaxation, Gibbs distributions and the Bayesian restoration of images. IEEE Trans,XII Simpósio Nacional de Probabilidade e Estatística, Associação Brasileira de Estatística.

[27 ] Gelfand, A. E., e Dey, D. K. e Chang, H. (1992). Model determination using predictive distributions with implementation via sampling-based methods ( with discussion). In Bayesian Statistics 4 (J. M. Bernardo, J. O. Berger, A. P. Dawid e A. F. M. Smith, eds.,) pp. 147-167, Oxford University Press, Oxford.

[28 ] Gelfand, A. E. and Dey, D. K. (1994). Bayesian model choice: Asymptotics and exact calculations. Journal of the Royal Statistical Society., B, 56, 501-514.

[29 ] Gelfand, A. E. and Smith, A. F. M. (1990). Sampling-based approaches to calculating marginal densities. Journal of the American Statistical Association, 85, pp. 398-409.

[30 ] Gelman, A.; Rubin, D. B (1992). Inference from iterative simulation using multiple sequences (with discussion) Statistical Science. 7, pp. 157-511.

[31 Geweke, J. (1992). Evaluating the accuracy of sampling-based approaches to the calculation of posterior moments (with discussion). In Bayesian Statistics 4, J. M. Bernardo, J. O. Berger. A. P. Dawid and A.F.M. Smith (Eds.). 169-193. Oxford University Press, Oxford. 
[32 ] Goel, A. L., and Okumoto, K. (1979). Time dependent error detection rate model for software reliability and other performance measures. IEEE Transactions on Reliability., 28, 206-211.

[33 ] Jelinsky, Z. and Moranda, P. B. (1972). Prediction of Software Reliability Research In Statistical Computer Performance Evaluation, W. Freiberger, ed., New York: Academic Press, $465-497$.

[34 ] Leonard, T. (1978). Density estimation, stochastic processes and prior information (with discussion). Journal of Royal Statistical Society, Ser. B 40, $113-146$.

[35 ] Littlewood, B. and Verral, J. L. (1973). A Bayesian Reliability Growth Model for Computer Software. Applied Statistics. , 22, 332-346.

[36 ] Hasting, W. K. (1970). Monte Carlo sampling methods using Markov chains and their aplications. Biometrika, 57, 97 - 109.

[37 ] Hjort, U. (1980).A reliability distributions with increasing, decreasing, constant and bathtub failure rates. Technometrics,22 pp. 99-107.

[38 ] Kalbfleisch, J. D. and Struthers, C. A. (1982). An analysis of the Reynolds Metal Company data. Cana Journal New York: Academic.

[39 ] Karlin, S., and Taylor, H. M. (1975). A First Course in Stochastic Processes. New York: Academic.

[10 ] Kass, R. E. and Raftery, A. E. (1995). Bayes factor. J. Amer. Statist. Assoc., 90. pp. 773-795.

[11 ] Kass, R. E. e Wasserman, L. (1994). Formal rules for selecting prior distributions. Carnegie Mellon University,PA.

[12 ] Kuo. L. and Yang. T. Y. (1996). Bayesian computation for nonhomogeneous Poisson process in software reliability. J. Amer. Statist. Assoc., 91,pp. 763773. 
[43 ]Kuo, L., Peng, F (2000). A mixture - model approach to the analysis of survival data. Generalized linear models. Biostatistics, 5 , 5, 255-270.

[44 ]Kutoyants, Y. A (1984). Parametric Estimation for stochastic processes. Berlin: Herdermann-Verlag.

[45 ] Langberg, N. e Singpurwalla, N. D. (1985). A Unification of some Software Reliability Models. SIAM, Journal on Scientific an Statistical Computing., 6, $781-790$

[46 ] Mattews, D. E.; Farewell, V. T. (1982). On testing for a constant hazard against a change-point alternatives. Biometrics, 71, 299 - 304.

[47 ] Metropolis, N., Rosenbluth, A. W., Rosenblut, M. N., A.H., and Teller, E. (1953), Equation of state Calculations by Fast Computing Machines. Journal of chimical physics, 21, 1087 - 1092.

[48 ] Miller, R. G. (1981). Survival analysis, John Wiley \& Sons.

[49 ] Muralidharan, K. (1999). Testing for shape parameter in Weibull processes: A conditional Aproach. Statistical Methods. 1, 36-41.

[50 ] Muralidharan, K. (2001). On testing of parameters in modelated power law process. Applied Stochastic Models in Business and Industry. 17(4), 331-343.

[51 ] Muralidharan, K. (2002). On reliability inferences of i-th Weilbull process. Statistical Methods. 3(2), 50-63.

[52 ] Musa, J. D., Iannino, A. and Okumoto, K. (1987). Software reliability: measurement, prediction, aplication. New York: Mc.Graw Hill.

[53 ] Musa, J. D., and Okumoto K. (1984). A logarithmic Poisson execution time model for software reliability measurement. Proceedings Seventh International Conference on Software Engineering. Orlando. pp. 230-238.

[5.1 ] Nguyen, H. T.. Rogers. G. S. and Walker. E.A. (1984). Estimation in change-point hazard rate models. Biometrika. 71. 299-304. 
[55 ] Paulino, C. D.,Amaral Turkman, M. A. e Murteira, B. (2003). Estatística Bayesiana. Fundação Calouste Gulbenkian. Lisboa,

[56 ] Raftery, A. E. e Lewis, S. M. (1992). How many iterations in the Gibbs sampler? In Bayesian Statistcs 4 (J. M. Bernardo, J. O. Berger, A. P. Dawid e A. F. M. Smith, eds.), pp. 763-773,

[57 ] Raftery, A. E., Akman, V. E. (1986). Bayesian analysis of a Poisson process with a change-point. Biometrika, 73, 1, 85-89.

[58 ] Ramirez Cid, J. E.; Achcar, J. A. (1999). Bayesian inference for nonhomogeneous Poisson processes in software reliability models assuming monontonic intensity functions. Computational Statistics and Data Analysis, 32, 147-159.

[59 ] Ramirez Cid, J. E.; Achcar, J. A. (2002). "Software reliability considering the superposition of non-homogeneous Poisson processes in the presence of a covariate, Statistics, 36, 3, 259 - 269.

[60 ] Ripley, B. D. (1987). Stochastic simulation. J. Wiley, New York.

[61 ] Rodrigues, J., Cid, J. E. R. e Achcar, J. A. (2002). Bayesian analysis for the superposition of two dependent nonhomogeneous Poisson processes. Communications in Statistics. vol. 31, 9, pp. 1467-1478.

[62 ] Ross, S. M.(1996). Stochastic simulation. John Wiley Sons, Inc.

[63 ] Schwarz, G. (1978). Estimating the dimension of a model. Annals of Statistics. $1,111-127$.

[64 ] Smith, R. M. and Bain, L. J. (1975). An exponential power life-testing distribution. Communications in Statistics 469 - 481.

[65 ] Spiegelhalter, D. J. and Smith, A. F. M. (1982 0. Bayes factor for linear and loglinear models with vague prior information. J. Royal Statist. Soc., B , 44, pp. 377-387,

[66 ] Spiegelhalter, D. J., Thomas, A., Best, N. G. (1999).WingBugs: Bayesian inference using Gibbs Sampling Cambriclge: MRC Biostatistics Unit.

67 ] Spiegelhalter, D. J., Best. N. G., Carlin. B. P. e van der Linde. A. (2002). Bayesian measures of model complexity and fit (with cliscussion). J. Royal Statist. Soc.. B , 64. pp. 583-639. 
[68 ] Stone, M. (1974). Cross-validatory choice and assessment of statistical predictions (with discussion). J. Royal Statist. Soc., B , 36, pp. 111-147.

[69 ] Tanner, M., Wong, H. W. (1987). The calculation of posterior distribution by data augmentation Journal of the American Statistical Association., 82, pp. 528-540.

[70 ]Tierney, L. and Kadane, J. B. (1986). Accurate approximations for posterior momemts and marginal densities. J. Amer. Statist. Assoc., 81,pp. 82-86.

[71 ]Tierney, L., Kass, R. E., and Kadane, J. B. (1989). Approximate marginal densities for nonlinear functions. Biometrika., 76, 425-433. Corrections (1991)78, 233-234.

[72 ] Yang, T. Y. (1994). Computational appraches to Bayesian inference for software reliability, Ph.D theseis, Departament of Statistics, University of Connecticut, Storrs, U.S.A.

[73 ] Yang, T. Y., Kuo, L. (1999). Bayesian computation for the superposition Poisson process in software reliability . the Canadian Journal of Statistics., 27, 547-556. 